Linear and Non Linear Studies at RHIC Interaction Regions and Optical Design of the Rapid Cycling Medical Synchrotron

\author{
Javier Fernando Cardona \\ Brookhaven National Laboratory, Upton, New York
}

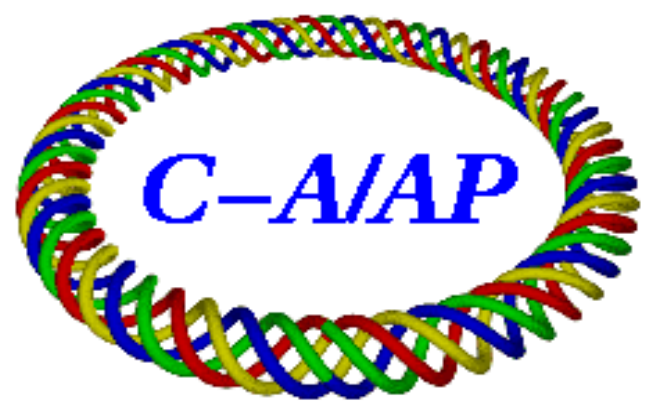

Collider-Accelerator Department Brookhaven National Laboratory

Upton, NY 11973 
Linear and Non Linear Studies at RHIC Interaction Regions and Optical Design of the Rapid Cycling Medical Synchrotron

A Dissertation Presented

by

Javier Fernando Cardona

to

The Graduate School

in Partial fulfillment of the

Requirements

for the Degree of

Doctor of Philosophy

in

Physics

State University of New York

at Stony Brook

August 2003 
Copyright by

Javier Fernando Cardona

2003 


\section{State University of New York at Stony Brook}

The Graduate School

\section{Javier Cardona}

We, the dissertation committee for the above candidate for the

Doctor of Philosophy degree,

hereby recommend acceptance of this dissertation.

Steve Peggs, Advisor

Professor of Physics

\section{Michael Rijssenbeek, Chairman of the Defense \\ Professor of Physics}

\section{Jacobus Verbaarschot, Committee member \\ Professor of Physics}

\section{Jim Murphy, Committee member Associate Chair for Accelerator, NSLS, BNL}

This dissertation is accepted by the Graduate School

Dean of the Graduate School 
Abstract of the Dissertation

\title{
Linear and Non Linear Studies at RHIC Interaction Regions and Optical Design of the Rapid Cycling Medical Synchrotron
}

\author{
by \\ Javier Fernando Cardona \\ Doctor of Philosophy \\ in \\ Physics \\ State University of New York \\ at Stony Brook \\ 2003
}

Development and application of the action and phase technique used to evaluate and correct local errors, linear and non linear (skew quadrupole errors, gradient errors and sextupole errors), at RHIC interaction regions is presented in the first part of this thesis.

The skew quadrupole errors have their origin on the roll angles of the quadrupoles. It is then possible to estimate the skew quadrupole error present in a RHIC triplet if all the roll angles of the quadrupoles of a particular triplet are known. These values were estimated with the measured roll angles during the 2002 RHIC shutdown period and compared to the measured skew quadrupole errors obtained with the action and phase technique. The agreement is fairly good validating the action and phase technique for at least skew 
quadrupole errors.

Another way of validating the action and phase technique is by intentionally introducing known values of errors while attempting to measure the values with the technique. This was done for skew quadrupole errors and gradient errors with excellent results. Analysis of some of the experiments shows that the set errors can be reproduced by the technique with accuracies below 5 percent. Same experiments were repeated for sextupole errors an a clear correlation between the measured and the set error was found but the precision in this case is not as good as for the linear errors case.

The optical design of the Rapid Cycling Medical Synchrotron and related efforts to optimize the design are presented in the second part of this thesis. An interesting outcome of this work is the development of the so called IBEFUMFO technique which allow a better understanding of the optical parameters involved in a lattice design and hence facilitate the task of the designer.

The rapid repetition frequency of the RCMS has raised concerns about the sextupole components induced in the beam due to strong Eddy currents. Tracking simulations with Marylie have been done in order to evaluate the effect of these sextupoles in quantities like the dynamic aperture and the footprint of the accelerator. 
To my mother 


\section{Contents}

Contents vi

List of Figures $\quad$ xii

List of Tables $\quad$ xxvi

Preface $\quad x \times x i$

Acknowledgments $\quad$ xxxvi

1 Basic Accelerator Optics 1

1.1 Introduction . . . . . . . . . . . . . . . . 1

1.2 Equations of Motion ................ . . 3

1.3 Solution to Betatron equations . . . . . . . . . . . . . . 10

1.4 Matrix Formalism of the Betatron Motion . . . . . . . . . . . 11

1.5 Magnet Errors and Closed Orbits . . . . . . . . . . . . . 14

1.6 Quadrupole or Linear Errors . . . . . . . . . . . . . . . . . . . 18

1.7 Beta-Beat Modulation . . . . . . . . . . . . . 19

1.8 Linear Coupling . . . . . . . . . . . . . . . . . 20 
1.8.1 Coupling from Rotated Quadrupoles . . . . . . . 20

1.8.2 Coupling from Solenoidal Fields . . . . . . . . . . 26

1.8.3 Global Coupling: $\Delta q_{\min } \ldots \ldots . . \ldots 27$

2 Action and Phase Jump Analysis 31

2.1 The Method .................... 34

2.2 Application of Action and Phase analysis to RHIC 2000 First turn Orbits . . . . . . . . . . . . . . . . 35

3 Linear Errors at RHIC's IRs and First Turn Trajectory Analysis

3.1 Determination of Skew Quadrupole

Errors from Action and Phase Graphs . . . . . . . . . . . 43

3.2 RHIC Local Linear Coupling Correction System . . . . . . . . 47

3.3 Analysis of First Turn Simulated Trajectories . . . . . . . . . 49

3.4 RHIC 2000 Difference First Turn Trajectory Analysis . . . . . 52

4 Correction of Linear Coupling during the RHIC 2001 Run 57

4.1 Local Correction of Skew Quadrupole Errors at RHIC IRs . . 57

4.2 Global Correction of Linear Coupling . . . . . . . . . . . . . 60

5 Linear Errors from Closed Orbit Analysis 63

5.1 Closed Orbits and Action and Phase Analysis . . . . . . . . 64

5.2 Determination of Linear Errors from Individual Closed Orbits $\quad 70$

5.3 Simulation of Gradient and Skew Errors . . . . . . . . . . . 73 
5.4 Dipole Correctors and IR errors . . . . . . . . . . . . . . . 75

5.5 Effect of Nonlinear Errors in the Determination of Linear Errors 78 5.5.1 Experiment .................. 78

5.5.2 Analysis . . . . . . . . . . . . . . . . . . . 79

5.5.3 Results for Proton Experiments . . . . . . . . . 85

5.5.4 Results for Gold Ion Experiments . . . . . . . . . . 89

5.5.5 Tune correction of the lattice model . . . . . . . . . . 93

5.6 Comparison between RHIC Run 2000 and RHIC Run $2001 \quad$ • 95

6 Skew Error Measurements and Roll Angle Measurements 101

6.1 Introduction . . . . . . . . . . . . . . . . 101

6.2 Skew Error Measurements and $2 \phi$

Hypothesis . . . . . . . . . . . . . . . . . 102

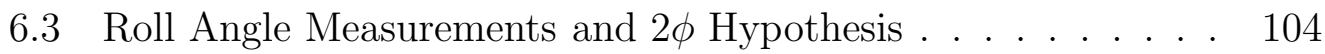

$\begin{array}{llr}7 & \text { Nonlinear Errors } & 113\end{array}$

7.1 The Method . . . . . . . . . . . . . . . 113

7.2 Experiment to Find non Linear Errors . . . . . . . . . . . . . 115

7.2.1 Analysis ................... 116

7.2.2 Results..................... 120

8 Calibration Experiments $\quad 125$

8.1 Calibration Experiments for Skew

Quadrupole Correctors . . . . . . . . . . . . . . 125 
8.2 Calibrating Gradient Error

Measurements ................... . . 126

8.3 Sextupole Corrector Calibration Experiment . . . . . . . . . 130

9 Software for Analysis of First Turn Orbits and Closed Orbits 135

9.1 Preprocessing the Orbit Data . . . . . . . . . . 135

9.2 The Main Program . . . . . . . . . . . . . . . . . . . . . . . 138

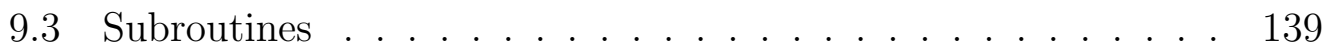

9.3.1 Subroutine GETBETA . . . . . . . . . . . . . 139

9.3.2 Subroutine GETPOSX and GETPOSY . . . . . . . . . 140

9.3.3 Subroutine INITIATEPOS . . . . . . . . . . . . . . . 140

9.3.4 Subroutine PRINCIPALX and PRINCIPALY . . . . . 141

9.4 Auxiliar Software . . . . . . . . . . . . . . . . . 145

10 Concluding Remarks 146

11 Proton Therapy 150

11.1 Introduction . . . . . . . . . . . . . . . . 150

11.2 Physics of Proton Therapy . . . . . . . . . . . . . . . . 151

11.3 Dosimetry of Proton Therapy . . . . . . . . . . . . . . . . 155

12 General Design Considerations for the Rapid Cycling Medical $\begin{array}{ll}\text { Synchrotron (RCMS) } & 157\end{array}$

12.1 General Requirements of a Therapy Facility . . . . . . . . . . 157

12.2 Design Choices . . . . . . . . . . . . . . . . 158 
13.1 The Basic Formulas of IBEFUMFO $\ldots \ldots \ldots$

14 Lattice Design of the RCMS 173

14.1 Ring Optics Design . . . . . . . . . . . . . . . . . . . 174

14.2 Injection $\ldots \ldots \ldots \ldots \ldots \ldots$

14.3 Extraction . . . . . . . . . . . . . . . . . 187

14.4 Delivery System Optics . . . . . . . . . . . . . . . . . . . . 192

14.4.1 Tolerances of the New Ring . . . . . . . . . . 198

14.5 Magnet Considerations . . . . . . . . . . . . . . . . . 198

14.6 Comparisons with Previous Designs . . . . . . . . . . . 202

14.7 Comparisons with other Medical Accelerators . . . . . . . 203

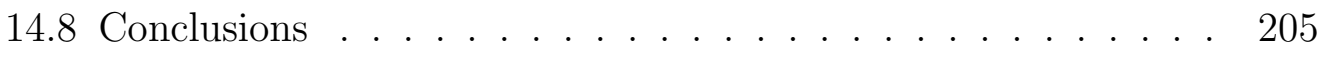

15 Effect of the Eddy Current in the beam of the RCMS 207

15.1 Introduction . . . . . . . . . . . . . . . . 207

15.2 Magnetic Multipoles Generated by Eddy Currents . . . . . . . 207

15.3 Marylie Simulations . . . . . . . . . . . . . . . . . . . 211

15.3.1 Preparation of the Lattice and Main Dipole Splitting . 212

15.3.2 Effect of the Sextupole Components on the Dynamic Aperture of the RCMS Ring . . . . . . . . . . . 215

15.3 .3 Footprints . . . . . . . . . . . . . . 220

15.4 Conclusions . . . . . . . . . . . . . . . . . . 220 
A Multipolar Expansion

B Equivalent Multipolar Coefficients in RHIC IRs

B.1 Calculation of the Equivalent Skew

Quadrupole for a RHIC triplet . . . . . . . . . . . . . 228

B.2 Calculation of the Equivalent Skew

Quadrupole for a RHIC IR . . . . . . . . . . . . . . . . 230

B.3 Calculation of the Equivalent Quadrupole Gradient for a RHIC

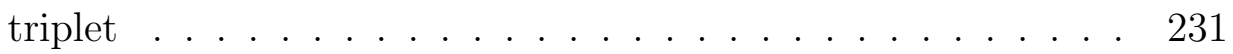

B.4 Calculation of the Equivalent Normal

Sextupole for a RHIC triplet . . . . . . . . . . . . . . 233

B.5 Calculation of the Equivalent Skew Sextupole for a RHIC Triplet236

B.6 Equivalent Octupole Coefficients . . . . . . . . . . . . . . 236

B.7 Multipolar Expansion for an Equivalent Magnetic Kick in a RHIC Triplet . . . . . . . . . . . . . . . . 238

C Fits of Orbit Data to the Betatron Equation in RHIC Arcs 240

$\begin{array}{ll}\text { D The Orbit Bump Method } & 245\end{array}$

E Common problems found in the Action and Phase Analysis of $\begin{array}{lr}\text { Difference Orbits } & 249\end{array}$

F Skew Quadrupole Correctors 253 


\section{List of Figures}

1.1 a) Schematic of the dipole magnet. b) Schematic of the quadrupole magnet. ........................ 2

1.2 Trajectory A remains inside the magnet for a longer length and hence this trajectory is more bended than trajectory $\mathrm{B}$. The net effect is that the trajectories A and B approach to each other after they pass the magnet (focusing effect). . . . . . . . . . 3

1.3 Focusing by the longitudinal fringe field in dipoles. a) Magnetic field lines on the plane perpendicular to the bending plane. b) Magnetic field in the bending plane. . . . . . . . . . . . . 4

1.4 Combined function magnets are dipole magnets with a quadrupole component created by tilting the magnet faces. . . . . . . . 5

1.5 Frame of reference for equation of motion. . . . . . . . . . . 6

1.6 Comparison of reference orbit path length $d s$ and particle path length $v d t[2] . \ldots \ldots \ldots \ldots$

1.7 Betatron orbit with magnetic error at $s=0$. It is shown that $x^{\prime}\left(s_{0}-\epsilon\right)=x^{\prime}\left(s_{0}-\epsilon+2 \pi Q\right) \ldots \ldots \ldots 16$ 
1.8 Deflection of a particle trajectory by a short magnet with mag-

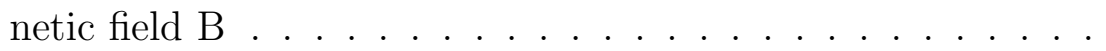

1.9 The direction of the magnetic forces in 4 different points of a quadrupole magnetic field. . . . . . . . . . . . . . . . . . . 22

1.10 Measurement of $\Delta q_{\text {min }}$ from coupled tunes. . . . . . . . . . . . 29

2.1 a) Action analysis of an orbit calculated with the betatron equation. b) Phase analysis of the same orbit. c) Representation of the RHIC lattice. The long bars represent quadrupoles while the short ones represent dipoles. . . . . . . . . . . . . .

2.2 a) Action analysis in the vertical plane of a difference trajectory obtained during the 2000 RHIC run. The oscillations were excited by a the dipole corrector bo7-tv7 located at $s=528.2 \mathrm{~m}$. b) Phase analysis of the same orbit. c) Lattice representation of RHIC. . . . . . . . . . . . . . . . .

2.3 a) Action analysis in the horizontal plane of a difference trajectory obtained during the 2000 RHIC run. In this case, the oscillations are induced from the vertical plane due to strong coupling at the IRs. b) Phase analysis of the same orbit. c) Lattice representation of RHIC. . . . . . . . . . . . . . . . 40 
2.4 principal window of the RHIC orbit display program. a) First turn orbit excited just before IR12. b) Lattice representation of RHIC. c) Induced orbit in the vertical plane due to coupling at IR's. . . . . . . . . . . . . . . . . .

3.1 a) Measured orbit (small circles) in the vertical plane fitted to the model (solid line) arc by arc. The action and phase of the model is obtained by averaging action and phases within each arc (see subroutine fitted $_{p}$ os in Appendix C). b) Lattice representation of RHIC. c) Action analysis of the measured orbit. d) Phase analysis of the measured orbit. . . . . . . . . . . . . 46

3.2 Horizontal beta functions used during the polarized proton RHIC run (Nov 302001 to Jan 23 2002). Beta functions are significantly bigger at the interaction regions than in any other place of the ring. . . . . . . . . . . . . . . . . . . . . . . . . . . 48

3.3 Schematic of 8 o'clock RHIC IR. The beam position at a particular skew quadrupole corrector is determined with the closest BPM to such corrector in each triplet. As an example, the closest BPM to the left skew quadrupole corrector $(\mathrm{sq07c2b})$ in the figure is bo7-b3. If this BPM doesn't give a proper reading of the beam position, bo7-b1 is used instead. . . . . . . . . . 54 
4.1 Induced orbits in the horizontal plane after creating a vertical orbit bump around the right triplet of 8 o'clock. Setting the skew quadrupole corrector to the calculated value the rms of the induced orbit is about 3 times smaller. . . . . . . . . . 58

4.2 Horizontal and Vertical tunes while the skew quadrupole strength of family \#1 is varied ( Jun 4 RHIC 2001 run ). . . . . . . . . 61

5.1 a) Simulated RHIC orbit excited with a dipole corrector at $s=$ $215 \mathrm{~m}$ and with a magnetic kick error $\Delta x^{\prime}$ at $\mathrm{s}=1945 \mathrm{~m}$. b) Action analysis of the orbit in a). c) Phase analysis of the orbit

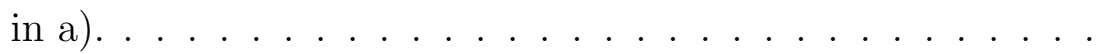

5.2 Relation between magnetic kicks and beam position. This data was taken in the right triplet of IR2 of the Blue ring. The different dots in all 3 lines correspond to different strength settings of the dipole corrector bo7-tv13. . . . . . . . . . . . 80

5.3 Relation between Magnetic kicks and Beam Position for Gold ions in triplet 7 of the Yellow ring when dipole corrector yi10th13 is used to shift the closed orbit. . . . . . . . . . . .

5.4 Relation between Magnetic kicks and Beam Position for Gold ions in triplet 7 of the Yellow ring when dipole corrector yi10tv12 is used to shift the closed orbit. . . . . . . . . 
5.5 a) Yellow ring difference orbit. b) Lattice representation of RHIC. c) Action analysis of orbit in a). d) Phase analysis of the orbit in a). The model (solid line in window a)) follows irregularly the BPM data and action and phase can hardly be considered as constant values. . . . . . . . . . . . . .

5.6 a) Difference orbit taken during the RHIC 2001 run. b) Lattice representation of RHIC. c) Action analysis of the orbit in a). d) Phase analysis of the orbit in a). The tilt on the curves of phase is due to difference between the real tunes of the machine and the tunes of the model. . . . . . . . . . . . . . . . . . . . . . . 94

5.7 a) Difference orbit taken during the RHIC 2001 run. b) Lattice representation of RHIC. c) Action analysis of the orbit in a). d) Phase analysis of the orbit in a).The tilt on the curves of phase gets significantly reduced when the horizontal tune of the model is changed from 28.22 to $28.12 \ldots \ldots$. . . . . . . 96

6.1 In order to cancel the field angle, the magnets are rotated opposite to the field angle when they are placed in the tunnel. It is possible that during this alignment procedure the magnets at the IR were rotated in the same direction that the field angle giving rise to a total roll angle equal to twice the field angle. . 103 
6.2 Skew errors from orbit measurements and skew errors calculated from the database field angles are compared. The measured errors were taken from Table 5.6 and Table D.1. . . . . . . . 105

6.3 a) Representation of a RHIC IR quadrupole. b) Configuration of the RHIC IR quadrupoles at any IR. The quadrupoles in the IRs are placed in the tunnel with their lead ends not always facing the same direction. . . . . . . . . . . . . . 106

6.4 a) Magnet located in clockwise direction (CW). b) Magnet located in counterclockwise direction (CCW). . . . . . . . . . 107

6.5 The roll angles of some of the IR quadrupoles were measured during the RHIC 2002 shutdown period and compared with the twice the field angles measured before the installation of the magnets. . . . . . . . . . . . . . . . . 108

6.6 The field angles were measured in the tunnel during the RHIC 2002 shutdown period by the magnet department at BNL. All the angles were measured from the Q1 no lead end and they are reported in the last column of the table (J. Animesh Courtesy). 109

6.7 The measured $A_{1}$ for all triplets of the Blue ring are compared with the calculated value that uses the measured roll angles. . 110

6.8 The measured values of $A_{1}$ found in Blue and Yellow triplets are compared with the corresponding values calculated from the measured roll angles, the 2 Field angle hypothesis and the value to which the skew quadrupole correctors were set. . . . . . . 112 
7.1 Graphs of magnetic kick vs beam position extracted from BPM measurements obtained by changing the strength of the horizontal dipole corrector bo6-th14. Even though the linear errors dominated these curves, nonlinear behavior are also present and it is possible to determine such nonlinearities from polynomial

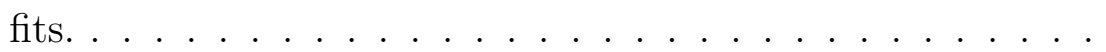

7.2 Graphs of magnetic kick vs beam position extracted from BPM measurements obtained by changing the strength of the vertical dipole corrector bo6-tv17. Some of the points in these graph don't follow the expected linear trend of the curves in some cases due to poor fittings of the original orbits. . . . . . . . . 118

8.1 Calibration curve for the right skew quadrupole corrector (bi8qS3 or SQ08C2B) at IR8. . . . . . . . . . . . . . . . . . 127

8.2 The strength of bo7-qd1 is varied in the RHIC ramp editor manager while the equivalent gradient error $B_{1}^{x}$ is measured. . 128

8.3 Same as Fig. 8.2 but now measuring $B_{1}^{y}$. The precision of this experiment is better when compared to the previous case revealing the existence of a systematic error of about 0.1 in the slope of the curve. . . . . . . . . . . . . . . . . . . . . . 129 
8.4 Measurements of magnetic kick vs beam position at the location of the sextupole corrector used in the sextupole calibration experiment. Evaluation of $\chi^{2}$ for different sextupole strengths and polynomial fits makes evident the effect of the sextupoles in the orbit measurements. . . . . . . . . . . . . . 133

8.5 Sextupole calibration curve obtained with difference orbits collected during the RHIC 2003 dAu run. . . . . . . . . . . . 134

9.1 schematic diagram of the PRINCIPALX subroutine. PRINCIPALX and PRINCIPALY are the same except for the evaluation of the beta functions in each case. . . . . . . . . . . . . . . 142

9.2 Convention used by the main program to label the arcs and the 2 sides of each RHIC IR. Every step represents and arc which is labeled with an odd number. It is also illustrated how each side of the IRs is labeled. Average action and phase in each arc as well as associated quantities are labeled with the number that corresponds to their arc. The same is valid for the quantities associated with the triplet like skew quadrupole corrector strengths and beam position measurements at the skew quadrupole corrector. . . . . . . . . . . . . . . . . . 143

11.1 Dose deposition profile of a $190 \mathrm{Mev}$ proton beam in Tissue. The dose deposition near the skin is low compared to the deposition about $20 \mathrm{~cm}$ deeper. . . . . . . . . . . . . . . 152 
11.2 Range of protons in water as function of the incident proton kinetic energy. The behavior of the curve is almost linear in the range of interest. . . . . . . . . . . . . . . 153

11.3 Dose deposition for three different types of radiation: Electrons, $\mathrm{X}$-Rays and protons. The proton dose deposition is much narrower than the other 2 types. . . . . . . . . . . . . . 154

13.1 FODO cells structure of different lengths are matched by inserting two quadrupoles between them. (the narrow rectangles represent quadrupoles.) . . . . . . . . . . . . . 165

13.2 Symmetric FODO cell scheme made of a half defocusing quadrupole with focal length $\mathrm{f} / 2$, drift of Length $\mathrm{L}$, focusing quadrupole of focal length L, and half defocusing quadrupole of focal length $\mathrm{f} / 2.167$

13.3 The inter-space between quadrupoles of a FODO cell has a maximum for some value of the minimum beta function in the FODO cell . . . . . . . . . . . . . . . . . . 170

14.1 Perspective view of the racetrack layout of the synchrotron. . . 174

14.2 Plan view of the racetrack layout of the synchrotron. . . . . . 175

14.3 The arc is made out of 7 dipole which lead to a lattice with reflexion symmetry about the center of the arc. . . . . . . . . 177 
14.4 The application of IBEFUMFO easily allows to increase the length of the FODO cells in the straight section while keeping small the beta functions in the arcs. (the arcs can be identified by the wide rectangles that represent dipoles) . . . . . . . . 180

14.5 Lattice functions for the whole RCMS ring. . . . . . . . . . . . 181

14.6 Plan drawing of the synchrotron, showing a broad view of the injection interface with the tandem Van de Graaf injector, and the extraction interface with the first few switchyard elements.

14.7 Perspective views of the injection interface. . . . . . . . . . . 183

14.8 Schematic view of the interface between the tandem Van de Graaf injector and the synchrotron. . . . . . . . . . . . . 183

14.9 Plan view of the interface between the tandem Van de Graaf injector and the synchrotron. . . . . . . . . . . . . 184

14.10Nominal trajectory of beam passing through the injection inflector. (Note that beam enters from the left, in this sketch.) . 184

14.11Plan view of the electrostatic injection inflector. Beam enters at the right from the tandem Van de Graaf injector. . . . . . . 188

14.12 Apertures at downstream end of electrostatic inflector. . . . . 188

14.13 The green and blue ellipses in the schematic represent $\pm 2.5 \sigma$ (rms) of the beam envelope at the upstream and downstream ends of the inflector. . . . . . . . . . . . . . . . . 188

14.14 Perspective view of extraction (foreground) and injection (background) interfaces. . . . . . . . . . . . . . . . . . 189 
14.15 Schematic view of the extraction interface layout. . . . . . . . 189

14.16 Plan view of the extraction interface layout. . . . . . . . . . 189

14.17 Nominal extraction trajectory of the beam leaving the synchrotron. . . . . . . . . . . . . . . . . . . 191

14.18 Extraction Septum Magnet . . . . . . . . . . . . . . 192

14.19 Plan view of the upstream end of the septum magnet, and septum magnet apertures. . . . . . . . . . . . . . 193

14.20 Schematic of the upstream end of the septum magnet, and septum magnet apertures. . . . . . . . . . . . . . . . 193

14.21 The interface between the extraction line and the switch yard simultaneously suppress the dispersion initiated by the septum magnet and smoothly matches the beta functions to the FODO cells structure of the switch yard. . . . . . . . . . . . . 195

14.22 The distances and strengths of 3 quadrupoles before the rotation point are set such that the alpha functions are equal to zero and the beta functions are equal in both planes. . . . . . 196

14.23 The achromatic gantry is built with seven 30 degrees dipole. Dispersion is match such that it is zero at the gantry output. . 197

14.24 The trajectories and beam pipes of the switch yard magnet within the iron of the 22.5 degrees dipole showing that the beam can go through the magnet even when it is off. . . . . . . . . . 200 
14.25 The trajectory of the beam when the $22.5^{\circ}$ magnet is used as $6.5^{\circ}$ magnet. The $6.5^{\circ}$ trajectory fits loosely within the pipe of the magnet. . . . . . . . . . . . . . . . . 201

15.1 The main dipoles are divided in $\mathrm{n}$ sector bends and the multipolar elements are inserted between them. The edges of the magnet are carefully modeled with general bending magnets that allow individual pole phase rotation of the faces of the magnet. Fringe fields in the edge are also added. . . . . . . . . 213

15.2 Computation of the fractional difference of beta functions between MAD and Marylie. The black line corresponds to the fractional difference of the horizontal beta functions while the red line corresponds to the fractional difference of the vertical beta functions. . . . . . . . . . . . . . . 216

15.3 Horizontal phase space ellipses at one location of the ring after tracking for 62530 turns with different integrated sextupole strengths. The nominal sextupole strength of $0.522 \mathrm{~T} / \mathrm{m}$ is represented by the red ellipse. . . . . . . . . . . . . . . 217

15.4 Horizontal phase space ellipses at one location of the ring after tracking for 62530 turns with different integrated sextupole strengths. The nominal sextupole strength of $0.522 \mathrm{~T} / \mathrm{m}$ is represented by the red ellipse. . . . . . . . . . . . . . . . 218 
15.5 Amplitude distribution of particles before doing tracking to evaluate tune footprints. . . . . . . . . . . . . . . 221

15.6 Horizontal tune distribution of the particles after tracking for 62530 turns. . . . . . . . . . . . . . . . . . 222

15.7 Vertical tune distribution of the particles after tracking for 62530

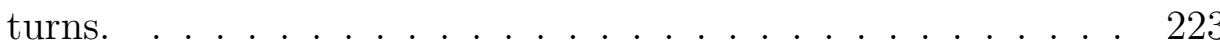

C.1 The orbit data (weak signals)in the arc that goes from IR8 to IR10 of the previous figure is compared with the modeled orbits obtained with three different methods. . . . . . . . . . . . 243

D.1 a) A closed orbit bump about the left triplet of IR8 is built in the horizontal plane. b) Lattice representation of RHIC. c) Coupling in the triplet induces an orbit oscillation in the vertical plane. The rms value of this betatron oscillation can be used to calculate the skew quadrupole error. . . . . . . . . . . 246

E.1 a) Orbit measurements and corresponding modeling in two RHIC arcs. Each arc is fitted independently. b) Lattice representation of two RHIC arcs. The arcs correspond to the group of bars at each side of the smaller group which, in turns, represent the triplet magnets at the IR's. c) Action analysis of the orbit in a). d) Phase analysis of the orbit in a). . . . . . . . . 251 
E.2 a) Detail of a difference orbit showing all six BPMs used at the 8 o'clock RHIC IR. The central BPMs (bxs) are necessary to make individual estimates of the triplet errors. The b3s BPMs are used to estimate the beam positions at the correctors. If any of these BPMs fails, the closer b1 will be used instead. b) Left and right quadrupole triplets of the 8 o'clock RHIC IR (long bars).The short bars represent the Dx magnets used to control the collision of the beams. c) Action analysis of the orbit in a). b) Phase analysis of the orbit in a). . . . . . . . . . . 252

F.1 View of the magnetic lines of a focusing quadrupole rotated 45 degrees in the counterclockwise direction from the downstream face of the magnet. . . . . . . . . . . . . . . . . . 254 


\section{List of Tables}

1.1 Phenix solenoids parameters. Some of the parameters were taken from $[31] \ldots \ldots \ldots \ldots$

3.1 Three different approaches were used to find $y^{s c}$ in the program MODULAR_IR2 ...................... 51

3.2 Measured Quadrupole Skew Errors(Run 2000) in Blue ring. More than 40 difference trajectories with kicks at different positions were used to obtain this table . . . . . . . . . . 55

4.1 Skew quadrupole correctors at IR8, IR10 and IR2 were set by using the orbit bump method on line. Since the settings obtained in this way were consistent with the measured values, the remaining correctors were set in one step according the measured values. In order to compare the second and the third column of this table it is neccesary to add the strengths of the left and right triplets of each IR. . . . . . . . . . . . . . 
5.1 skew errors from closed orbit analysis (RHIC 2000 orbits). No gradient errors are considered for this analysis. . . . . . . . .

5.2 Skew and Gradients obtained from Closed Orbit Analysis. Orbits taken during the 2001 RHIC run. . . . . . . . . . . . . . 72

5.3 Effect of the difference phase advance between the dipole corrector used to excite the betatron oscillation and the IR under study in the determination of linear errors. diff_phase is not exactly the phase advance between corrector and and IR but rather it is a normalized number that indicate how close to the IR the closed orbit has a maximum (see Chapter 9). All the orbits were simulated with $A_{1}=0.0011 / \mathrm{m}$ and $B_{1}=-0.001$ $1 / \mathrm{m} \ldots \ldots \ldots \ldots \ldots \ldots \ldots \ldots \ldots \ldots \ldots \ldots$

5.4 Closed orbits with skew errors in the right triplet of IR10 were simulated and MODULAR_IR2 was then used to extract the errors from the simulated orbits. As before the linear errors used in the simulation were $A_{1}=0.0011 / \mathrm{m}$ and $B_{1}=-0.001$

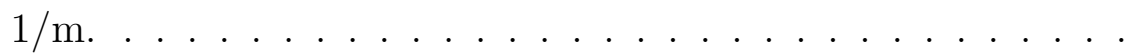

5.5 Sample of error analysis done with protons orbits at the left triplet of IR8 in the Blue ring. . . . . . . . . . . . . .

5.6 Linear errors in the Blue ring triplets. Proton orbits were used

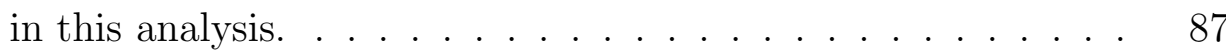

5.7 Linear errors in Yellow Ring Triplets. Proton orbits were used in this analysis. . . . . . . . . . . 
5.8 Error analysis in Blue ring assuming that the gradients are the same in both planes . . . . . . . . . . . . . . . 88

5.9 Linear errors in some of the Yellow triplets obtained from gold ion difference orbits. When only difference orbits created with one corrector are available for a specific IR Eq. 5.17 is used

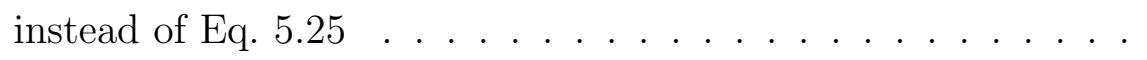

5.10 Linear Errors from difference orbits in the Blue ring. The model has been re-tuned from $\mathrm{Qx}=28.22$ to $\mathrm{Qx}=28.12$. . . . .

5.11 Comparison between skew error measurements done in the Blue ring during the run 2000 vs the corresponding measurements done during the run 2001 (All values are given in $10^{-3} 1 / \mathrm{m}$ ). .

5.12 Strengths of the Triplet Quadrupoles at IR6 and IR10. The third column corresponds to the nominal strength of the quadrupoles used in the Blue ring during the Gold run in 2000. The fourth column corresponds to the nominal strengths used in the Blue ring during the proton run in 2001. . . . . . . . . . . . 99

5.13 Calculated skew quadrupole errors from Eq. B.6 for the Gold lattice used during the RHIC 2000 run and the proton lattice used during the RHIC 2001 run. The roll angles were assumed to be twice the field angles measured before the magnets were installed in the tunnel (see Chapter 6). . . . . . . . . . . . . 100 
7.1 Linear and Quadratic Coefficients after fitting data to Eq. 7.5 and Eq. 7.6. Linear coefficients are given in $\mu \mathrm{rad} / \mathrm{mm}$ while the quadratic coefficients are given in $\mu \mathrm{rad} / \mathrm{mm}^{2}$. . . . . . . . 121

7.2 Equivalent Multipole Coefficients. The units are $\frac{\mu \mathrm{rad}}{\mathrm{mm}}$ or $10^{-3} \frac{1}{\mathrm{~m}}$ for the linear coefficients and $\frac{\mu r a d}{m^{2}}$ or $\frac{1}{m^{2}}$. . . . . . . . . 124

12.1 Primary parameters of the Rapid Cycling Medical Synchrotron

14.1 Relation between the magnets needed in the arc and their lengths to always produce a total bend of 180 degrees with the same magnetic field . . . . . . . . . . . . . . 176

14.2 Basic matching parameters in the straight section of the RCMS ring. . . . . . . . . . . . . . . . . . . 177

14.3 Injection Kicker and Inflector Parameters . . . . . . . . . . . . 185

14.4 Extraction Kicker and Septum Magnet Parameters . . . . . . 190

14.5 A variation of the quadrupole strengths by $2 \%$ lead to the changes shown on the table. The variations are considered insignificant for operations purposes. . . . . . . . . . . . 198

14.6 Comparison between the number of magnets needed before and after doing the trajectory studies. Those studies show that C-type magnets and 6.5 degree magnet can be replaced with combined function magnets. . . . . . . . . . . . . . . 202

14.7 Cost change between some of the systems of the old design of the RCMS and the corresponding ones to the most recent design.202 
14.8 Magnet Pole Gap and Magnet Pole Tip Width for 3 Different Facilities. . . . . . . . . . . . . . . .

15.1 Sextupole components induced by Eddy currents in an elliptical beam pipe for different values of the major semi-axes $a$. . . 209

15.2 Quadrupoles strengths of the multipoles inserted in the main dipoles used in the Marylie Software. Direct conversion from the MAD lattice leads to tunes that differ slightly from the design ones. Readjustment of the quadrupole strengths in the third significant figure are then neccesary to bring back the tunes to their original values. . . . . . . . . . . . . . . . . . . 214

15.3 The radius $r$ for which particles start to get lost in the tracking simulations is found as function of the sextupole strength. The chromaticities $Q x^{\prime}$ and $Q y^{\prime}$ are also calculated in each run. . . 219

C.1 Effectiveness of the fit for 3 different procedures on the same orbit data. . . . . . . . . . . . . . . . . . . . 244

D.1 Calculated IR skew corrector strengths. . . . . . . . . . . . . 247

E.1 Preliminary analysis of difference orbits used to evaluate triplet errors at 8'o clock IR. Only analysis in the horizontal plane are shown. . . . . . . . . . . . . . . . . . . . . 249 


\section{Preface}

\section{Part I: Linear and Non Linear Studies at RHIC Interaction Regions}

One of the fundamental quantities of an accelerator is the luminosity. The interaction regions or the places where the experiments are placed in the accelerator should be designed to maximize the luminosity. From the optical point of view, this means that the beam must be as small as possible at the interaction point. This can be achieved by a special group of magnets placed at each side of the interaction point. These magnets have relatively high magnetic fields when compared with the regular magnets used in other places of the accelerator. As a consequence, any misalignment of the interaction region magnets as well as any defect in their magnetic fields have a bigger impact in the performance of the accelerator than misalignments or defects in the regular magnets. Traditionally, global correction methods have been used to correct for the errors all around the ring with satisfactory results in most of the cases. However when strong errors are localized in particular places like the interaction regions the global compensation methods are less robust and 
difficult to implement during machine operation.

In order to circumvent this problem, accelerator like RHIC and the LHC have implemented packages of multipole correctors in the interaction regions to perform local compensation besides the traditional global compensation methods.

The values at which these local corrector must be set are expected to be determined by either magnetic measurements or beam based measurements.

The beam based measurements are preferred over the magnetic measurements because the first ones allow to evaluate the field errors under the current conditions of the machine. Several techniques have been proposed to perform the error measurements based on beam observables and such techniques are currently under development. The first part of this thesis show the development and implementation of the orbit action jump technique to measure local coupling errors, gradient errors and nonlinear errors at the interaction regions. This technique has been successfully used in RHIC to compensate local coupling errors at the interaction regions and there are solid experimental evidences that this technique can precisely evaluate integrated gradient errors at RHIC interaction regions.

Chapter 1 introduces some elementary concepts in accelerator physics that will be used throughout this thesis. Even though most of the results shown in this Chapter are well known in the field there are few results that were derived during the course of this work and that are fundamental for the results shown in the next chapters. Chapter 2 describes the basics of the orbit action jump 
technique, its application to RHIC and some results that help to identify the Interaction Regions as the source of linear coupling during the 2000 run.

The quantitative determination of skew quadrupole errors from the action and phase variables obtained from first turn orbit analysis is presented in Chapter 3. Since the ultimate goal of the error measurements is to set up the correctors it is neccesary to introduce in this chapter the local correction system implented in some of the RHIC interaction regions.

Chapter 4 describes the local correction done during the RHIC 2001 run where the estimates for skew quadrupole errors done in the previous Chapter were used. Comparisons with the Orbit Bump method and evaluation of the minimum tune separation (a method widely used for coupling evaluation) indicates that the correction was successfully performed.

The action and phase analysis is extended to closed orbits in Chapter 5. A general method that precisely evaluates linear errors and that can be easily extended to measure nonlinear errors is also shown for the first time in this Chapter. Different species (protons and gold ions) are used to evaluate the errors. Comparisons between the errors found during the 2000 RHIC run ( Chapter 3) and the ones found during the 2001 run (this Chapter) are presented at the end of the chapter. During the the 2002 shutdown period the roll angles of some of the quadrupole of the triplets were measured. These measurements allowed the direct comparison of the skew quadrupole error measurements done in the previous chapters with the skew errors calculated directly from the measured angles. The results of this comparison (shown in 
Chapter 6) is completely positive strongly validating the orbit based measurements. The general method to evaluate linear error presented in Chapter 5 is extended to include also non linear errors in Chapter 7. Results of some of the experiments performed during the 2001 RHIC run suggest that it might be possible to extract at least sextupole component errors with the method presented in this Chapter.

The calibration Chapter (Chapter 8) the estimated values from the orbit values for known values of skew quadrupole strengths, gradient errors and normal sextupole strengths. The correspondence between the measured and the set values of skew and gradient errors is excellent (below 5\%). For the sextupole case there is a clear correlation between measured and set values but the precision of the measurements is not as good as for the linear case. Further studies and experimentation are needed to identify the sources of errors in this case. Chapter 9 is a brief description of the code and associated subroutines that were written to extract the action and phase variables from the orbit measurements, software for quantitative analysis, orbit preprocessing, etc.

\section{Part II: Optical Design of the Rapid Medical Synchrotron (RCMS)}

The Rapid Cycling Medical Synchrotron was initially conceived at FERMILAB in 1992 and after the University of Pennsylvania shows serious intentions of acquiring a proton therapy facility, a collaboration with center at Brookhaven National Laboratory (BNL) was established between Advanced 
Energy Systems (AES), Lawrence Berkeley Laboratory (LBL), Stony Brook University, ACCEL in Germany and BNL. The output of this collaboration was initially a preconceptual design in 1999, an internal review in January of 2002 and a conceptual design in March of 2003.

This part of the thesis is the contribution to the RCMS collaboration in the optical design and associated efforts to reduce the cost of the facility.

Chapter 11 is a brief introduction to proton therapy. The general requirements and the design choices of the RCMS are explained in detail in Chapter 12 .

One of the interesting outcomes of the optical design of the RCMS was the development of the so called IBEFUMFO technique which became an important tool for optimization. The principle and development of this technique is explained in Chapter 13.

The whole optic design is presented in Chapter 14. It includes the optical design of the ring, delivery lines, the gantry optical interface: a special optical device developed for the RCMS, the injection and extraction systems, applications of the IBEFUMFO technique and comparisons with other accelerators.

The effect of the eddy currents due to the fast repetition frequency of the RCMS is discussed in Chapter 15. These studies are aimed to determine if sextupole correctors are needed in the accelerator. 


\section{Acknowledgments}

I consider myself very fortunate of having landed in the collider accelerator department at Brookhaven National Laboratory for my doctoral research. It is a very active place with plenty of possibilities for research and with an excellent scientific staff. I personally benefit from the interaction with many of the scientists of the department to whom I would like to thank.

I'm very thankful to Todd Satogata for helping me to start up with my equipment, for help on the beam experiments done at RHIC and for continuously solving many of my computer related problems.

I want to express my appreciation to Vadim Ptitsyn with whom I team up to do the linear studies at RHIC Interaction Regions and with whom I held frequent conversations that allow me to move easily in my RHIC studies. Dan T Abell did also a significant contribution to my research. He sat down with me for several weeks and introduce me to Marylie, the simulation program based on Lie Algebra developed by Alex Dragt, thesis advisor of Dan during his graduate studies. I particularly enjoy many of the conversations that we had about non linearities in accelerators and its connection with Marylie. I'm in debt with Dan for the precious help received on the research of Eddy currents 
in the RCMS which is presented on Chapter 15.

I have the opportunity to interact with Kip Gardner, particularly in the optics design of the RCMS lattice. This design was in part shaped by his valuable comments and the pressure he was putting to improve his design of the injection and extraction systems. I owe to him the sections of injection and extraction presented on Chapter 14 of this dissertation. Jorg Kewisch Steve Peggs and I sat down in several occasions to discuss the many issues of the RCMS lattice. I collected many good ideas from that discussions and I also obtained several software tools developed by Jorg to assist lattice design. I found these tools very useful in the lattice design of the RCMS.

Many thanks to Fulvia Pilat for the space that she gave to perform my experiments in the beam experiments section that she coordinates and for the many opportunities I had to show my work in her weekly meeting. I also appreciated the confidence she put on me to present the RHIC Interaction Region Studies in the 2001 snowmass conference.

Many Thanks to Dejan Trbojevic that also allow me to show my work several times in the commissioners meeting, provide me with critical data about the rolls of the quadrupoles at the Interaction Regions and stimulated the work related with RHIC that I present in this dissertation.

Many Thanks to Steven Tepikian who helped me to solve several MAD related problems and RHIC lattice related problems. Many Thanks to Johannes Van Zeijts for the assistance given to plan several of the beam experiments presented in this dissertation. I also want to thank all the administrative 
staff specially Mary Campbell, Marion Heimerle and Gladys Blas who always attend promptly all my required paper work for travels, housing ,etc.

I also want to express my gratitude and appreciation to Ray Fliller and Vajid Ranjbar my former officemates with whom I interchange all spectrum of ideas, physics related and non physics related making my days at the office more enjoyable. I also found valuable the company and input of my later officemates Ubaldo Iriso and Rama Calaga.

Many thanks to Rogelio Tomás García who made valuable comments in some of the chapters of this dissertation.

I am very thankful to my advisor ,Steve Peggs, definitively the person with whom I interact the most during my stay at the lab and the person responsible for shaping most of my extraordinary experience at BNL. I'm not going to enumerate all the activities in which I received his guidance support and encouragnent because I will end up with a very long list. All of the above and more describes briefly and precisely the work that we have done together. I also enjoy been part of the Fulbright program in the US. I have the opportunity to attend many of their meetings and special programs. These events were always full of friendly faces, excellent organization, and interesting amenities.

Many thanks to Matthieu Delecroix, Francisco Martinez and Diana Hernandez. They were supportive friends upon my arrival to Stony Brook.

I would specially like to thank Pat Peiliker who diligently took care of many of the formalities that the university required from me during my stay 
at Stony Brook.

I would like to thank the financial and administrative support provided by the Institute of International Education (IIE) through the Fulbright program, Colciencias and "Universidad Nacional de Colombia" in the first part of my program of studies and then the financial and administrative support provided by Stony Brook University and Brookhaven National Laboratory in the later part of my program of studies. 


\section{Part I: Linear and Non Linear Studies at RHIC Interaction Regions}




\section{Chapter 1}

\section{Basic Accelerator Optics}

\section{$1.1 \quad$ Introduction}

The acceleration of particles usually requires the confinement of the particles in a small space generically called beam. Confinement of the beam is usually achieved by dipole and quadrupole magnets placed in a configuration called strong focusing. The dipole magnets define the ideal orbit of the particle while the quadrupole magnets keep the beam focused and as close as possible to the ideal orbit. Dipole magnets have a constant vertical magnetic field (Fig. 1.1 a)) that produces a force that bends the particle in the horizontal plane. Quadrupole magnets (Fig. 1.1 b)) produce magnetic fields that vary linearly with the position of the beam with respect to the center of the quadrupole. Such magnetic fields are characterized by a constant $B^{\prime}$ called the magnetic gradient. Dipole magnets also have quadrupole components which can focus the beam either horizontally or vertically. In a sector bend magnet (see Fig. 1.2) for example, there is a net horizontal quadrupole effect. This is 
a)
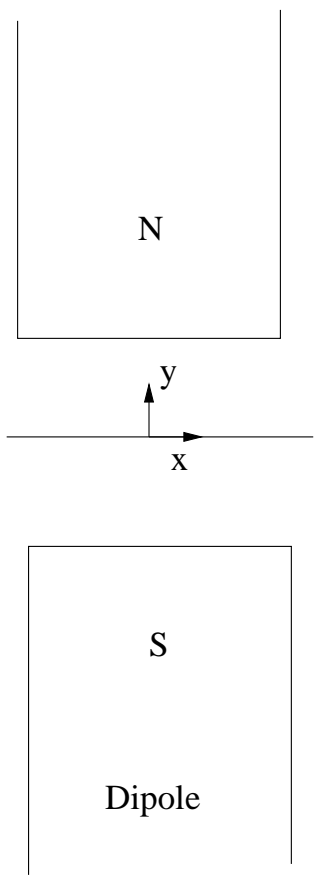

b)

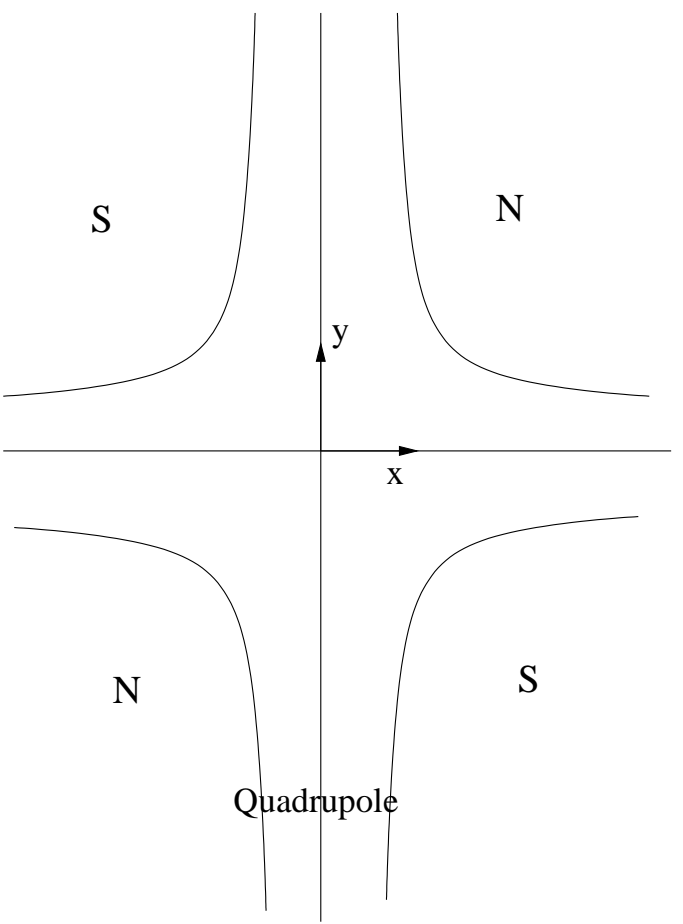

Figure 1.1: a) Schematic of the dipole magnet. b) Schematic of the quadrupole magnet.

due to the fact that particles that enter the magnet at different heights respect the central line of the magnet travel different distances inside the magnet.

Vertical quadrupole effects are produced by the irregular pattern of the magnetic field at the edge of the magnet (see Fig. 1.3). This effect appears only when $\alpha$, the angle between the magnet's end face and the perpendicular to the beam direction, is different from zero (see Fig. 1.3). The focusing system of the first accelerator was based on this effect giving place to what is know as weak focusing.

Strong quadrupoles can also be added to a dipole by tilting the inner faces 


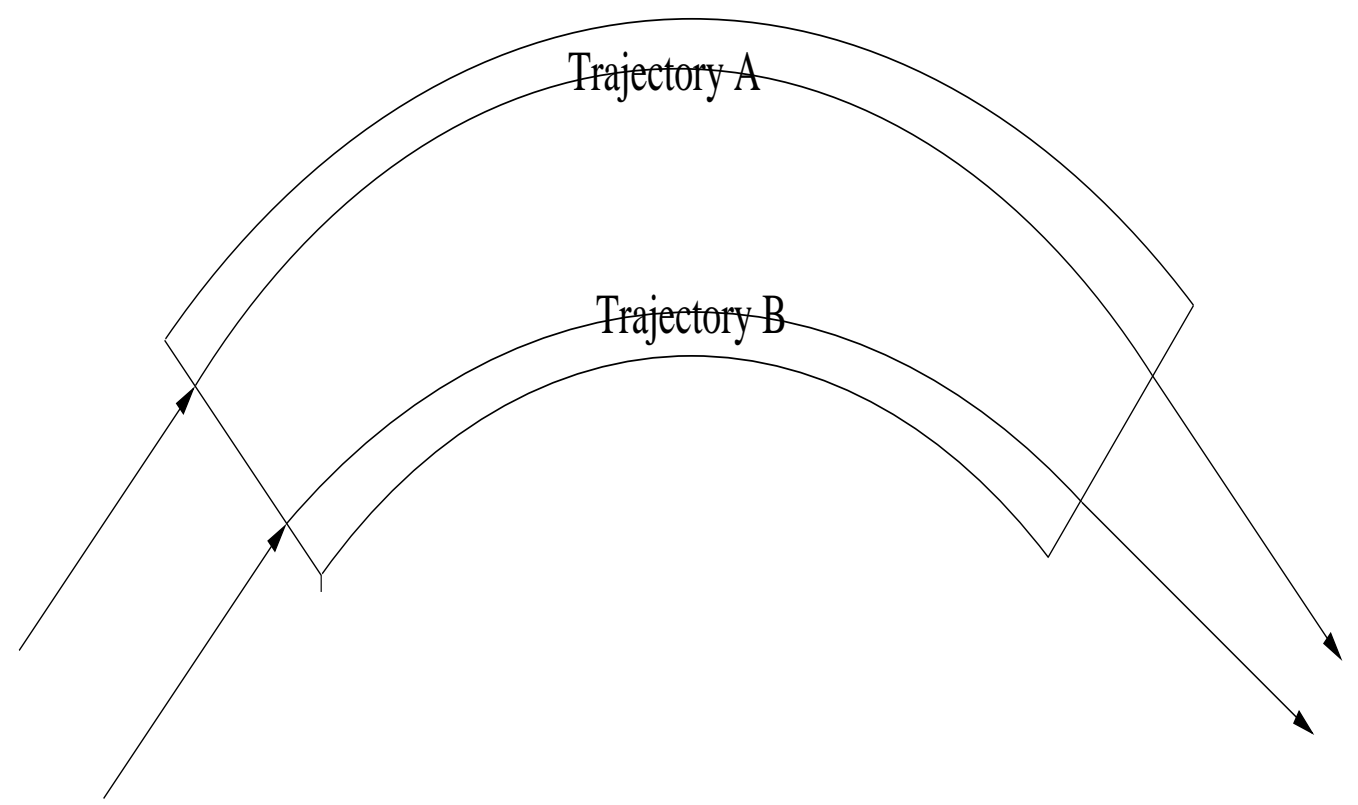

Figure 1.2: Trajectory A remains inside the magnet for a longer length and hence this trajectory is more bended than trajectory $\mathrm{B}$. The net effect is that the trajectories $\mathrm{A}$ and $\mathrm{B}$ approach to each other after they pass the magnet (focusing effect).

of the magnet like shown in Fig. 1.4. These magnets are known as combined function magnets and they can be generally found in small accelerators.

\subsection{Equations of Motion}

This section contains a mathematical description of the particles in a strong focusing accelerator with specific mathematical derivations that are used in the analysis of some of the experiments described in this thesis work.

The reference frame chosen for the study of the motion, Fig. 1.5, is also found in [2], although the mathematical derivation here is slightly different. 

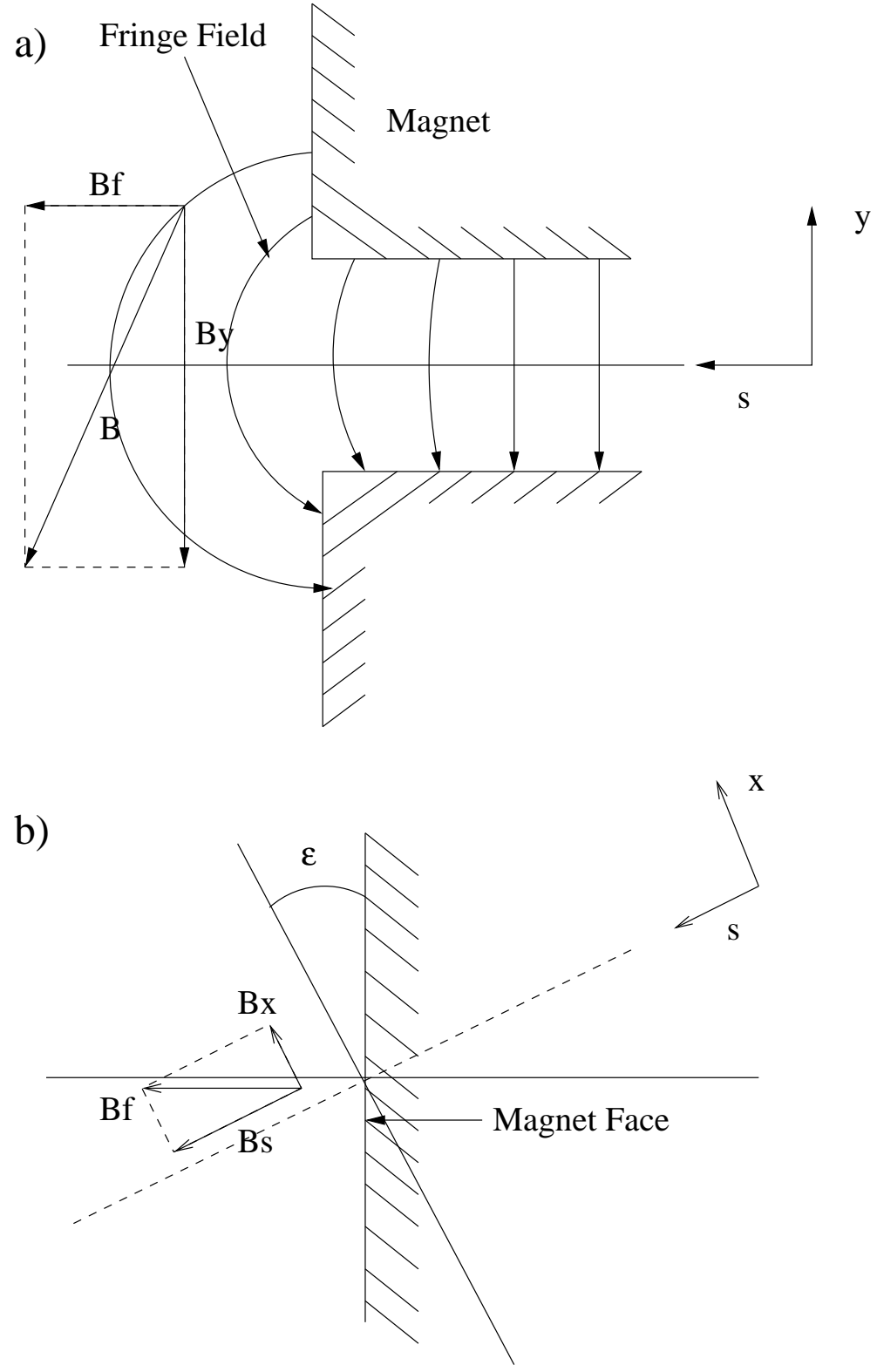

Figure 1.3: Focusing by the longitudinal fringe field in dipoles. a) Magnetic field lines on the plane perpendicular to the bending plane. b) Magnetic field in the bending plane. 


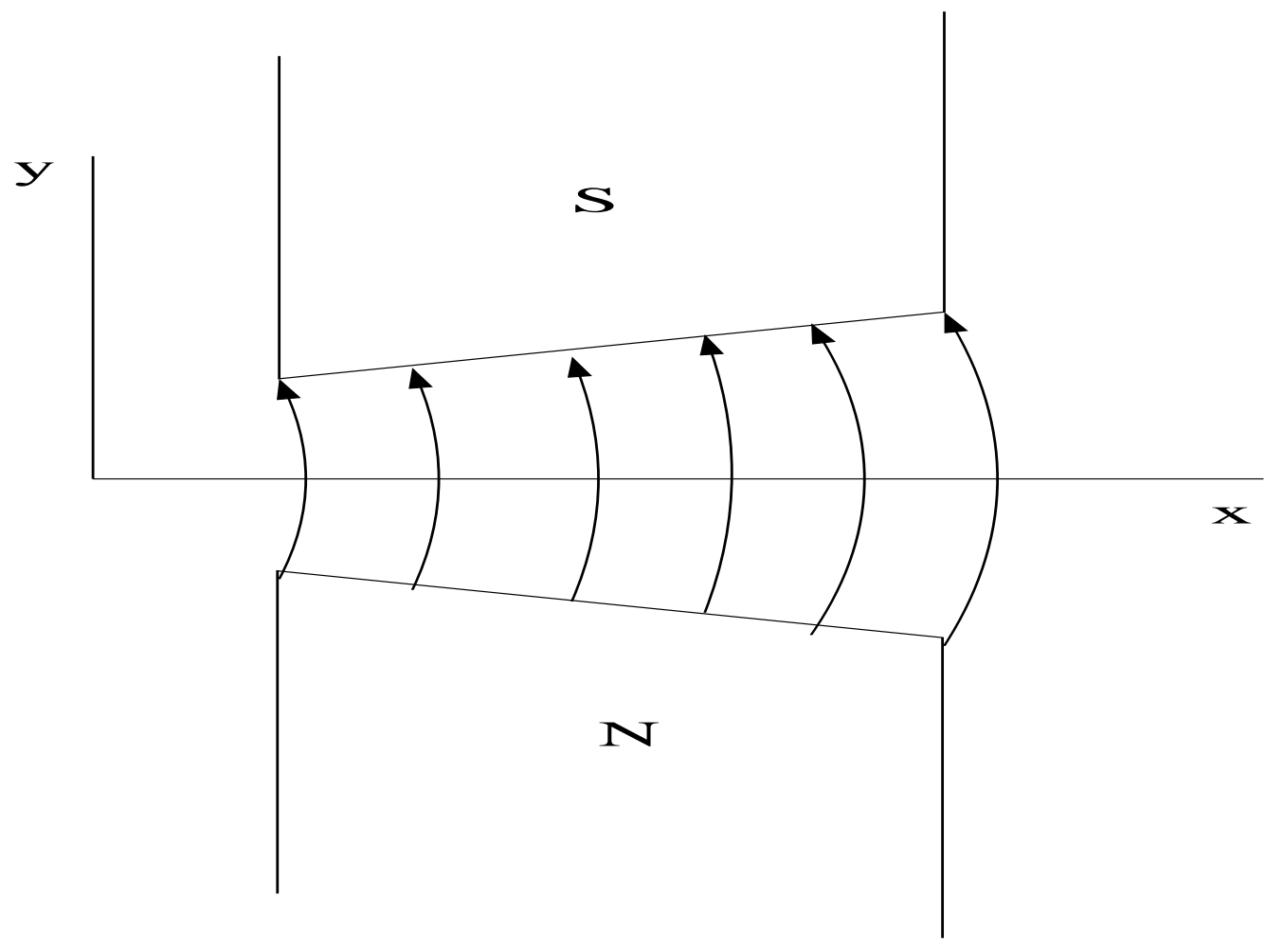

Figure 1.4: Combined function magnets are dipole magnets with a quadrupole component created by tilting the magnet faces.

The motion is generally divided into longitudinal and transverse components. The longitudinal motion is described by the axial coordinate "s" while the transverse motion is described by the " $\mathrm{x}$ " and " $\mathrm{y}$ " coordinates. The longitudinal motion depends mainly on radio frequency $(\mathrm{RF})$ cavities used for acceleration while the transverse motion depends on the magnetic optics of the accelerator. In this section, only transverse motion will be described. To simplify the algebra, the equations will be written from the non-inertial reference frame $x$ y $s$ which requires to add a centrifugal force in the $+x$ direction 


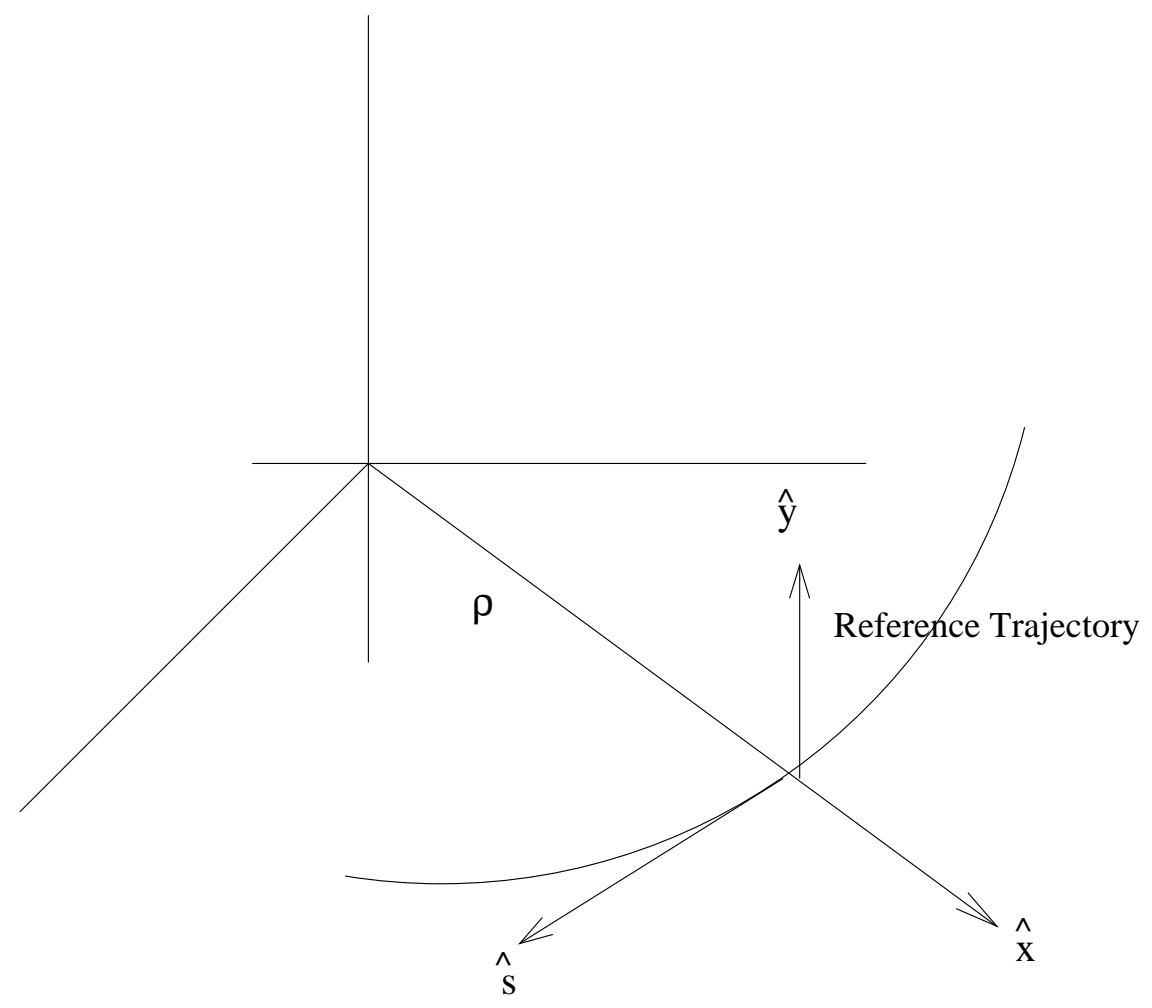

Figure 1.5: Frame of reference for equation of motion.

(see equation 6.20 in [3]). The equations of motion are then:

$$
\frac{d \vec{p}}{d t}=q \vec{v} \times \vec{B}+\vec{F} \text { centrifugal }
$$

Where the $q$ is the charge of the particle, $v$ its longitudinal velocity and $\vec{B}$ a generic magnetic field. Horizontal and vertical components of Eq. 1.1 are:

$$
\begin{aligned}
\frac{d}{d t}\left(\gamma m \frac{d x}{d t}\right) & =-q v B_{y}+\text { Fcentrifugal } \\
\frac{d}{d t}\left(\gamma m \frac{d y}{d t}\right) & =q v B_{x}
\end{aligned}
$$


where $m$ is the mass of the particle and if only dipole and quadrupole magnetic fields are assumed and no radiation effects are considered ( $\gamma$ is constant), it follows from Eq. 1.2 that:

$$
\begin{aligned}
& \gamma m \frac{d^{2} x}{d t^{2}}=-B_{y} v q+\gamma m \frac{v^{2}}{\rho+x} \\
& \gamma m \frac{d^{2} y}{d t^{2}}=B_{x} v q
\end{aligned}
$$

where $B_{x}$ is the horizontal component of the magnetic field, $B_{y}$ is the vertical component of the magnetic field, $v$ is the longitudinal velocity of the particle, $\rho$ is the radius of the central orbit, and $q$ is the charge of the particle.

Eq. 1.3 must be given as function of the azimuthal coordinate $s$ rather than the time $t$. The following relations can be extracted from Fig. 1.6:

$$
\begin{aligned}
d s & =\rho d \theta \\
v d t & =(\rho+x) d \theta
\end{aligned}
$$

which lead to:

$$
d s=\rho d \theta=v d t \frac{\rho}{\rho+x}
$$

where $v$ is the velocity of the particle along $s$. Here we assumed that the transverse velocities are very small when compared with $v$. It is now necessary to express the derivatives of time as derivatives of $s$. The chain rule of the calculus allow us to write:

$$
\frac{d^{2} x}{d t^{2}}=\left(\frac{d s}{d t}\right)^{2} \frac{d^{2} x}{d s^{2}}+\frac{d^{2} s}{d t^{2}} \frac{d x}{d s}
$$




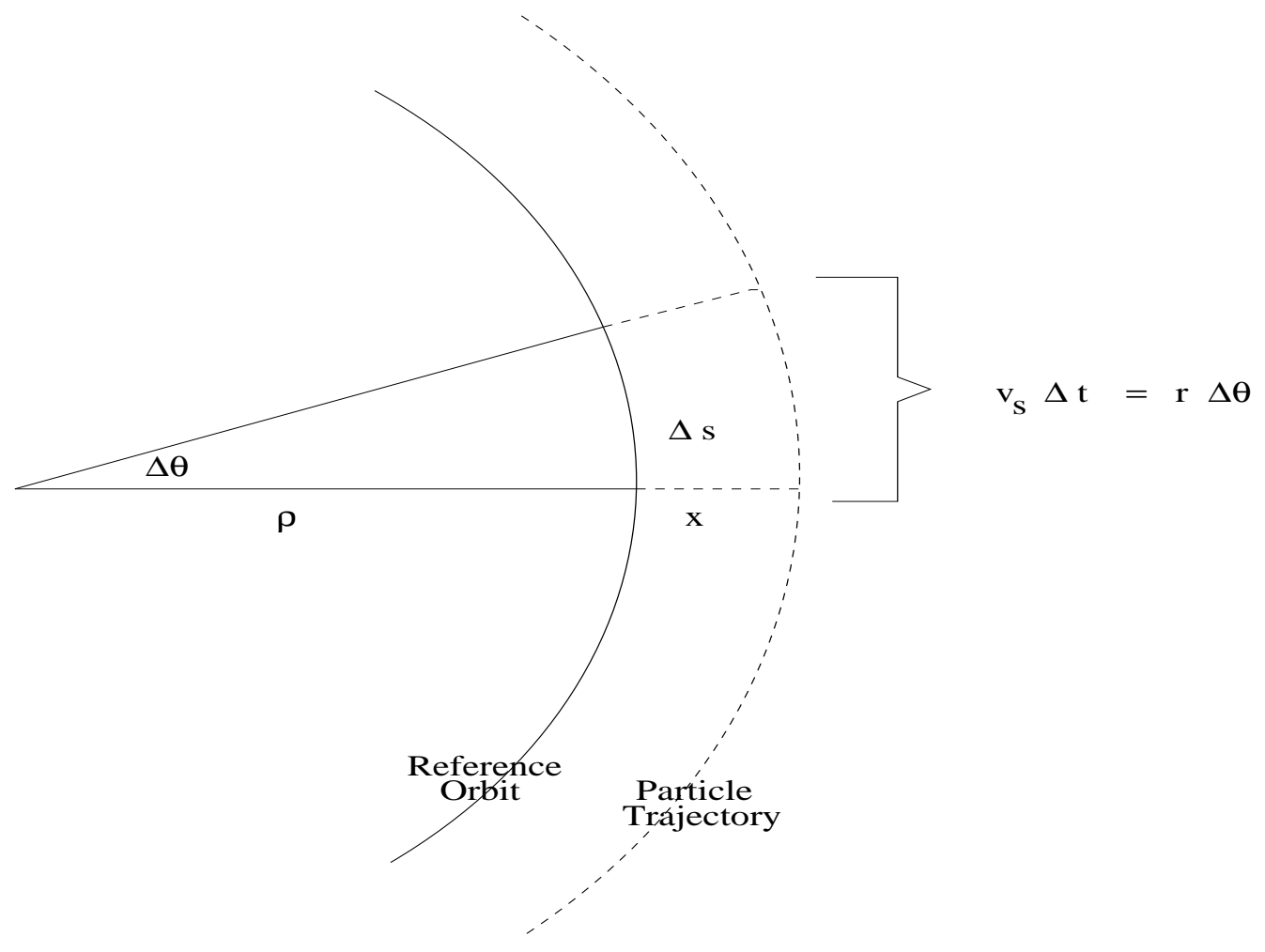

Figure 1.6: Comparison of reference orbit path length $d s$ and particle path length $v d t[2]$.

$$
\begin{aligned}
& =\left(\frac{v \rho}{\rho+x}\right)^{2} \frac{d^{2} x}{d s^{2}}+\frac{d x}{d s} \frac{v \rho}{\rho+x} \frac{d x}{d t} \\
& =\left[\frac{d^{2} x}{d s^{2}}+\left(\frac{d x}{d s}\right)^{2}\right]\left(\frac{v \rho}{\rho+x}\right)^{2}
\end{aligned}
$$

where Eq. 1.5 has been used to obtain the first and second derivative of $s$ vs $t$.

The quadratic term in Eq. 1.7 can be ignored since $\frac{d x}{d t}$ is a small quantity. In such case:

$$
\frac{d^{2} x}{d t^{2}}=\frac{d^{2} x}{d s^{2}}\left(\frac{v \rho}{\rho+x}\right)^{2}
$$


Similarly for the y plane we have:

$$
\frac{d^{2} y}{d t^{2}}=\frac{d^{2} y}{d s^{2}}\left(\frac{v \rho}{\rho+x}\right)^{2}
$$

Replacing Eq. 1.7 and Eq. 1.8 in Eq. 1.3 and assuming that $x^{2}$ is negligible respect $\rho^{2}$ we have:

$$
\begin{aligned}
\frac{d^{2} x}{d s^{2}}+\frac{1}{\rho^{2}} x+\frac{1}{B \rho}\left(B_{y}-B_{0}\right) & =0 \\
\frac{d^{2} y}{d s^{2}}-\frac{B_{x}}{B \rho} & =0
\end{aligned}
$$

where $B_{0}$ is the dipole magnetic field that only have a vertical component and $B \rho$ is what is known as the magnetic rigidity and it is defined by:

$$
B \rho=\frac{\gamma m v}{q}
$$

The magnetic fields can be expressed in function of the quadrupole gradients:

$$
\begin{aligned}
B_{x}(s) & =B^{\prime}(s) y \\
B_{y}(s)-B_{0} & =B^{\prime}(s) x
\end{aligned}
$$

Using these results in Eq. 1.9 we have:

$$
\begin{aligned}
\frac{d^{2} x}{d s^{2}}+\left[\frac{1}{\rho^{2}}+\frac{B^{\prime}(s)}{B \rho}\right] x & =0 \\
\frac{d^{2} y}{d s^{2}}-\frac{B^{\prime}(s)}{B \rho} y & =0
\end{aligned}
$$


Both differential equations horizontal and vertical can be generically written as:

$$
z^{\prime \prime}+K_{z}(s) z=0
$$

where $z^{\prime \prime}=\frac{d^{2} z}{d s^{2}}$ and $z$ can be either $x$ or $y$. Eq. 1.13, generally called Hills equation, is similar to the harmonic oscillator equation except that $K_{z}$, the spring constant, depends on the azimuthal coordinate $s$. Transverse motion described by Eq. 1.13 is usually known as betatron motion.

\subsection{Solution to Betatron equations}

Since Eq. 1.13 resembles the harmonic oscillator equation with a variable spring constant it is natural to propose a solution of the form:

$$
x(s)=A w(s) \sin [\psi(s)+\delta] .
$$

Substitution of this solution in the Hills equation leads to equations that relate $\psi(s)$ and $w(s)$ :

$$
\begin{aligned}
\psi^{\prime} & =\frac{k}{w(s)^{2}}, \\
w^{3}\left(w^{\prime \prime}+K w\right) & =k^{2}
\end{aligned}
$$

where $k$ is a constant of integration. The solution of these equations gives a complete description of the transverse motion of a single particle. Usually $w(s)$ and its derivative are written in terms of the so called Courant-Snyder 
parameters [1]:

$$
\begin{aligned}
\beta(s) & \equiv \frac{w^{2}(s)}{k} \\
\alpha(s) & \equiv-1 / 2 \frac{d \beta(s)}{d s}=-1 / 2 \frac{d}{d s}\left(\frac{w^{2}(s)}{k}\right), \\
\gamma(s) & \equiv \frac{1+\alpha^{2}}{\beta} .
\end{aligned}
$$

The solution of the equations of motion with the above definitions now has the form:

$$
x(s)=\sqrt{2 J \beta(s)} \sin [\psi(s)+\delta]
$$

where $J$ and $\delta$ are constant of motion that depend on the initial conditions. The constant $J$ is usually referred as the action of the motion.

Eq. 1.17 can also be used to describe the motion of the beam envelope of a bunch of particles. In such a case the amplitude of the oscillation is related with the beam size $\sigma$ by the equation:

$$
\sigma=\sqrt{\epsilon \beta}=\sqrt{<J>\beta}
$$

where the emittance of the beam $\epsilon$ has been defined as the average action of all particles within the bunch.

\subsection{Matrix Formalism of the Betatron Mo- tion}

It is common to find problems in which a particle starting with phase space coordinates $x_{0}$ and $x_{0}^{\prime}$ goes trough a beam line and the final space coordinates 
must be determined. The turn by turn matrix $T$ is a very useful tool to solve those kind of problems. In general, $T$ can be written as:

$$
\begin{aligned}
\left(\begin{array}{c}
x \\
x^{\prime}
\end{array}\right)_{s_{0}+C} & =T\left(\begin{array}{c}
x \\
x^{\prime}
\end{array}\right)_{s_{O}} \\
& =\left(\begin{array}{cc}
\cos \Delta \psi_{C}+\alpha \sin \Delta \psi_{C} & \beta \sin \Delta \psi_{C} \\
-\gamma \sin \Delta \psi_{C} & \cos \Delta \psi_{C}-\alpha \sin \Delta \psi_{C}
\end{array}\right)\left(\begin{array}{c}
x \\
x^{\prime}
\end{array}\right)_{s_{O}}
\end{aligned}
$$

where $\Delta \psi_{C}$ is usually expressed as $2 \pi Q_{0}$ where $Q_{0}$ is the tune of the machine. Beam lines are usually divided in small parts each of one is characterized by its own matrix $M_{i}$. The total Matrix $T$ for a beam line containing $n$ elements line can then be written as:

$$
T=M_{n} \ldots M_{i} \ldots M_{0} .
$$

Some of the typical $M$ matrices are:

$$
M_{\text {drift }}=\left(\begin{array}{cc}
1 & L \\
1 & 0
\end{array}\right)
$$

for a drift of length $\mathrm{L}$ and

$$
M_{\text {quad }}=\left(\begin{array}{cc}
1 & 0 \\
k l & 1
\end{array}\right)
$$

for a short quadrupole of integrated strength $k l$.

As example we will calculate the tune shift $\Delta Q$ that a thin quadrupole gradient error $k l$ produces on an otherwise perfect lattice. The stability conditions in the tunes will also be discussed. The optical matrix for a lattice perturbed by a thin quadrupole gradient error $k l$ is given by [20]:

$$
T=\left(\begin{array}{cc}
\cos 2 \pi Q_{0}+\alpha \sin 2 \pi Q_{0} & \beta \sin 2 \pi Q_{0} \\
-\gamma \sin 2 \pi Q_{0} & \cos 2 \pi Q_{0}-\alpha \sin 2 \pi Q_{0}
\end{array}\right)\left(\begin{array}{cc}
1 & 0 \\
k l & 1
\end{array}\right)
$$


or

$$
T=\left(\begin{array}{cc}
\cos 2 \pi Q_{0}+\alpha \sin 2 \pi Q_{0}+\beta k l \sin 2 \pi Q_{0} & \beta \sin 2 \pi Q_{0} \\
-\gamma \sin 2 \pi Q_{0}+k l\left(\cos 2 \pi Q_{0}-\alpha \sin 2 \pi Q_{0}\right) & \cos 2 \pi Q_{0}-\alpha \sin 2 \pi Q_{0}
\end{array}\right) .
$$

Linear stability of the particle motion can be inferred from the trace of this matrix. Stability is ensured when [21] :

$$
-1 \leq \frac{1}{2} \operatorname{Tr} T \leq 1
$$

or

$$
\left|\cos 2 \pi Q_{0}+\frac{1}{2} \beta k l \sin 2 \pi Q_{0}\right|=\left|\cos 2 \pi\left(Q_{0}+\Delta Q\right)\right| \leq 1
$$

where $\left(Q_{0}+\Delta Q\right)$ is the tune value for the perturbed machine. The term $1 / 2 \beta k l \sin 2 \pi Q_{0}$ can make this inequality invalid, especially for values of $Q_{0}$ very close to one or a half integer. The machine is then in an unstable state known as the half integer resonance. If the tune shift $\Delta Q$ is small then,

$$
\cos 2 \pi\left(Q_{0}+\Delta Q\right) \simeq \cos 2 \pi Q_{0}-2 \pi \Delta Q \sin 2 \pi Q_{0}
$$

and then,

$$
\Delta Q=-\frac{1}{4 \pi} \beta k l
$$

The effect of several small gradient deviations can be written as:

$$
\Delta Q=-\frac{1}{4 \pi} \sum_{i}(\beta)_{i}(k l)_{i}
$$

Eq. 1.20 can also be written for values of $s$ different to $s_{0}+C$ (see for example [2]):

$$
T\left(s_{0}->s_{1}\right)=
$$




$$
\left(\begin{array}{cc}
\sqrt{\frac{\beta_{1}}{\beta_{1}}} \cos \Delta \psi+\alpha_{0} \sin \Delta \psi & \sqrt{\beta_{0} \beta_{1}} \sin \Delta \psi \\
-\frac{1+\alpha_{0} \alpha_{1}}{\sqrt{\beta_{1} \beta_{0}}} \sin \Delta \psi+\frac{\alpha_{0}-\alpha_{1}}{\beta_{1} \beta_{0}} \cos \Delta \psi & \sqrt{\frac{\beta_{0}}{\beta_{1}}}\left(\cos \Delta \psi-\alpha_{2} \sin \Delta \psi\right)
\end{array}\right)
$$

A problem of initial conditions that can be easily solved with the matrix formulation is when the beam is disturbed by a magnetic kick $\Delta x^{\prime}$ relative to the design trajectory. In such a case $x\left(s_{0}\right)=0$ and $x^{\prime}\left(s_{0}\right)=\Delta x^{\prime}$ which together with Eq. 1.31 leads to:

$$
x(s)=\Delta x^{\prime} \sqrt{\beta\left(s_{0}\right) \beta(s)} \sin \left(\psi(s)-\psi\left(s_{0}\right)\right)
$$

where $s_{0}$ is the place at which the $\Delta x^{\prime}$ is located. Eq. 1.31 is the basis for analysis of first turn orbits as we will see in the following chapters.

\subsection{Magnet Errors and Closed Orbits}

In circular accelerators, magnet errors like misalignments or magnetic field errors produces a net displacement of the so called closed orbit, a special orbit that closes in itself and around which the particles oscillate. Ideally the closed orbit corresponds to the design trajectory of the accelerator (usually $x(s)=0$ and $y(s)=0$ ). However, magnetic errors separate the design trajectory from the closed orbit in a way that can be predicted with the following equation:

$$
\begin{aligned}
x^{\prime \prime}+K_{x}(s) x & =-\frac{\Delta B_{y}(s)}{B \rho} \\
& = \\
y^{\prime \prime}+K_{y}(s) y & =\frac{\Delta B_{x}(s)}{B \rho}
\end{aligned}
$$


where $\Delta B_{x}(s)$ and $\Delta B_{y}(s)$ are the errors of the magnetic field in the horizontal and the vertical plane with respect to the design values. Eq. 1.32 can be easily inferred from Eq. 1.9.

In general, $\Delta B_{y}(s)$ and $\Delta B_{x}(s)$ can have normal multipolar components $a_{n}$ and skew multipolar components $b_{n}$ as indicated in the following equation.

$$
\Delta B_{x}^{\prime}-i \Delta B_{y}^{\prime}=B_{0} \sum_{0}^{\infty}\left(b_{n}+i a_{n}\right)(x+i y)^{n} .
$$

The usual problem in the machine optics is to have some few significant magnetic errors each with an effective length $\Delta l$ much smaller than the total ring length. This allows to write the magnetic errors as delta Dirac functions as follows:

$$
x^{\prime \prime}+K_{x}(s) x=-\sum_{i} \delta\left(s_{i}\right) \frac{\Delta B\left(s_{i}\right)}{B \rho} \Delta l=\sum_{i} \delta\left(s_{i}\right) \Delta x^{\prime}\left(s_{i}\right)
$$

where $s_{i}$ are the positions where the magnetic errors are localized $\Delta l$ is the length of the magnet and $\Delta x^{\prime}\left(s_{i}\right)$ represent the magnetic kicks at each $s_{i}$ position. The magnetic kick $\Delta x^{\prime}$ in general depends on $x\left(s_{i}\right)$ and $y\left(s_{i}\right)$ according to Eq. 1.33.

Notice that when $s_{i}<s<s_{i+1}$ Eq. 1.34 is equal to Eq. 1.13 and hence the beta functions found for the lattice without errors should also satisfy Eq. 1.15 which in turn means that the beta functions found for the lattice without errors can be used to model the real orbit in the intervals defined by the magnetic errors. This is valid despite the fact that errors might change the closed orbit beta functions. 
The general solution of Eq. 1.34 will be shown here for the case when only 1 magnetic error $\Delta x^{\prime}\left(x_{0}, y_{0}\right)$ is present.

The natural ansatz solution for Eq. 1.34 is Eq. 1.17. Since we are looking for a periodic solution equation $\left(x\left(\psi\left(s_{0}\right)\right)=x\left(\psi\left(s_{0}\right)+2 \pi Q\right)\right)$ and assuming $\psi\left(s_{0}\right)=0$ we have:

$$
\cos (-\delta)=\cos (2 \pi Q-\delta)
$$

One of the solutions of this equation lead to $\delta=\pi Q$. The other solution has

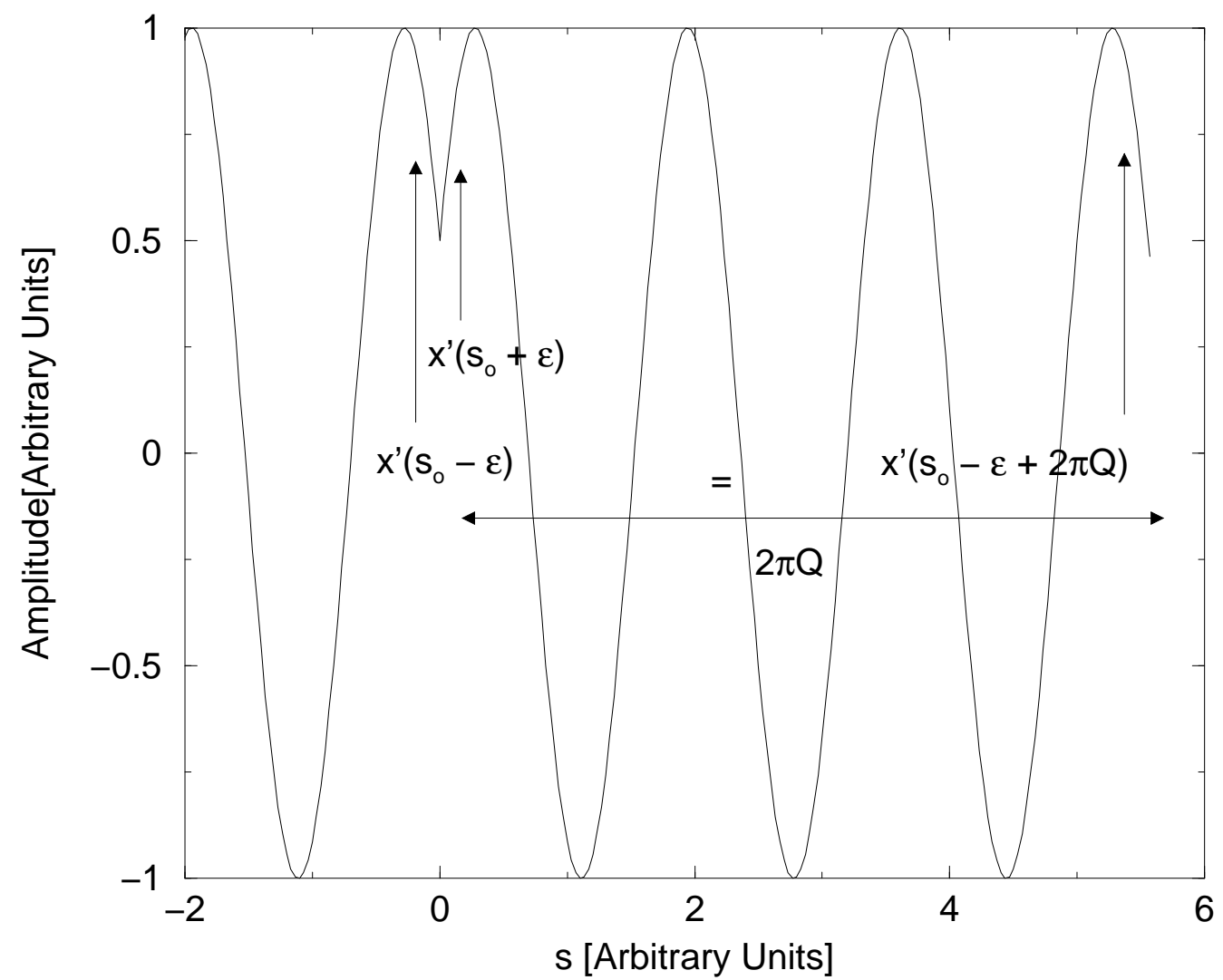

Figure 1.7: Betatron orbit with magnetic error at $s=0$. It is shown that $x^{\prime}\left(s_{0}-\epsilon\right)=x^{\prime}\left(s_{0}-\epsilon+2 \pi Q\right)$ 
an additional term $2 k \pi$ where $k$ is any integer number. The constant $\sqrt{2 J}$ can be found by first integrating Eq. 1.34 in the interval $s_{0}-\epsilon$ and $s_{0}+\epsilon$ where $\epsilon$ is and infinitesimally small number,

$$
\int_{s_{0}-\epsilon}^{s_{0}+\epsilon} x^{\prime \prime} d s+\int_{s_{0}-\epsilon}^{s_{0}+\epsilon} K(s) x d s=\int_{s_{0}-\epsilon}^{s_{0}+\epsilon} \delta\left(s_{0}\right) \Delta x^{\prime}\left(x_{0}, y_{0}\right) d s .
$$

After doing the integration we have:

$$
\lim _{\epsilon \rightarrow 0}\left(x^{\prime}\left(s_{0}+\epsilon\right)-x^{\prime}\left(s_{0}-\epsilon\right)\right)=\Delta x^{\prime}\left(x_{0}, y_{0}\right)
$$

where $x^{\prime}\left(s_{0}+\epsilon\right)$ is a limit coming from the right hand side and can be easily evaluated using the ansatz solution,

$$
\lim _{\epsilon \rightarrow 0} x^{\prime}\left(s_{0}+\epsilon\right)=-\frac{\sqrt{2 J}}{\sqrt{\beta\left(s_{0}\right)}}\left[\frac{1}{2} \cos (2 \pi Q)-\sin (\pi Q)\right],
$$

$x^{\prime}\left(s_{0}-\epsilon\right)$ on the other hand is a limit coming from the left hand side. In this case $\psi(s)$ must be changed to $\psi(s)+2 \pi Q$ (see Fig. 1.7) and then we have:

$$
\lim _{\epsilon \rightarrow 0} x^{\prime}\left(s_{0}-\epsilon\right)=-\frac{\sqrt{2 J}}{\sqrt{\beta(s)}}\left[\frac{1}{2} \cos (\pi Q)+\sin (\pi Q)\right] .
$$

Substituting the previous results in Eq. 1.37 it is possible to find the constant of the motion $\sqrt{2 J}^{1}$ as function of known parameters.

$$
\sqrt{2 J}=\frac{\Delta x^{\prime}\left(x_{0}, y_{0}\right) \sqrt{\beta}}{2 \sin (\pi Q)}
$$

which finally allows us to write the closed orbit equation as:

$$
x(s)=\frac{\Delta x^{\prime}\left(x_{0}, y_{0}\right) \sqrt{\beta}}{2 \sin (\pi Q)} \cos (\psi(s)-\pi Q)
$$

\footnotetext{
${ }^{1}$ In this case $J$ is a constant only in the intervals defined by the errors
} 
In general Eq. 1.41 should be written as:

$$
x(s)=\frac{\Delta x^{\prime}\left(x_{0}, y_{0}\right) \sqrt{\beta}}{2 \sin (\pi Q)} \cos \left(\left|\psi(s)-\psi\left(s_{0}\right)\right|-\pi Q\right)
$$

where $\psi\left(s_{0}\right)$ is the phase advance at the place where the magnetic kick $\Delta x^{\prime}$ is located.

If there is more than one magnetic kick in the ring the total closed orbit can be written as:

$$
x(s)=\sum_{i} \frac{\Delta x^{\prime}\left(x_{i}, y_{i}\right) \sqrt{\beta(s)}}{2 \sin (\pi Q)} \cos \left(\left|\psi(s)-\psi\left(s_{i}\right)\right|-\pi Q\right)
$$

\subsection{Quadrupole or Linear Errors}

Magnetic errors in an accelerator machine can be classified as dipole errors, quadrupole errors, sextupole errors and so on. The most common and stronger errors are usually the dipole and quadrupole errors. This dissertation is mainly concern with quadrupole errors ( we will also refer to them as linear errors) and they will be discussed in some detail in the following paragraphs.

There are 2 kinds of linear errors: normal gradient errors and skew quadrupole errors. Gradient errors appear for example when the real magnetic field of a quadrupole magnet is different from the design value. Skew

quadrupole errors appear when the quadrupole magnets are rolled about a longitudinal axis.

Gradients errors have undesirable effects like tune shifts already discussed in Section 1.4. Tune shifts can drive the machine to the so called tune reso- 
nances. Such tune resonances are predicted by Eq. 1.32 and they are basically tunes at which the beam amplitude grows without limit.

Another unwanted effect of the gradient errors is the so called beta-beat modulation which consist of oscillations of the beta functions over-imposed to the design beta functions. The most common effect of skew quadrupole errors is the so called linear coupling. Linear coupling can also be produced by solenoidal fields like the ones present in the experimental detectors. In the following sections we will briefly review all the mentioned effects.

\subsection{Beta-Beat Modulation}

The variation of the beta functions in the ring due to quadrupole gradient errors $\Delta k$ can be predicted with the formula:

$$
\Delta \beta(s)= \pm \frac{\beta(s)}{2 \sin \pi Q_{0}} \int_{s}^{s+C} \Delta k(\sigma) \beta(\sigma) \cos 2\left[|\mu(\sigma)-\mu(s)|-\pi Q_{0}\right] d \sigma
$$

A deduction of this equation can be seen for example in [22]. There the lattice is divided in 2 segments. One segment that goes from the observer position $s$ to the quadrupole error and other segment that goes from the quadrupole error position back to the observer. Three matrices can then be used to completely describe the lattice, one for each segment and the third for the quadrupole error. Comparison of the resultant matrix with general form matrix in function of the Courant Snyder parameters lead to Eq. 1.44.

Notice from Eq. 1.44 that a single quadrupole error can affect the beta functions all around the ring. Even though that is a valid mathematical de- 
scription of the effect of a quadrupole error in an optical lattice this description usually lead to complicated mathematical analysis.

Section 1.5 showed that it was possible to describe the particle orbit between errors while still using the unperturbed beta functions. This alternate mathematical approach significantly simplifies the error lattice description, as will be shown in the next chapters.

\subsection{Linear Coupling}

In principle, betatron oscillations in both transverse planes are independent of each other. However, rotated quadrupoles fields due to misalignment and occasionally solenoidal fields associated with high energy experiments can lead to some degree of coupling.

\subsubsection{Coupling from Rotated Quadrupoles}

In order to understand how the coupling arises in rotated quadrupoles I first analyze how the slope of the orbit ( $\mathrm{x}^{\prime}$ or $\mathrm{y}^{\prime}$ ) will change after crossing a quadrupole. According to Fig. 1.8 the geometrical relations between $\Delta x^{\prime}$, the angular deflection of the orbit respect its design orbit, $\Delta l$, the length of the magnet, and $\rho$, the radius of curvature of the trajectory through the magnetic field, is found to be:

$$
\Delta x^{\prime}=-\frac{\Delta l}{\rho}
$$




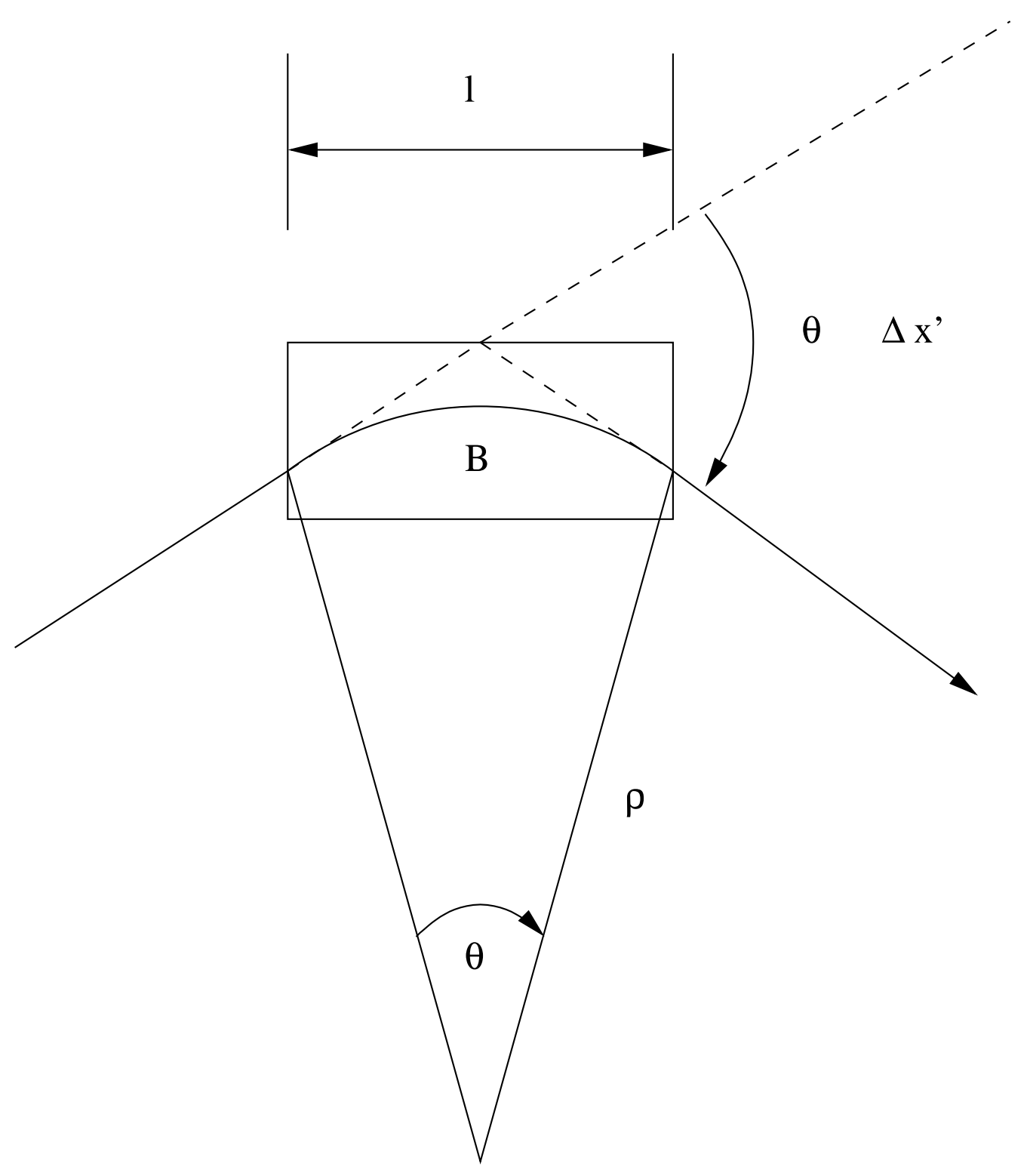

Figure 1.8: Deflection of a particle trajectory by a short magnet with magnetic field B 


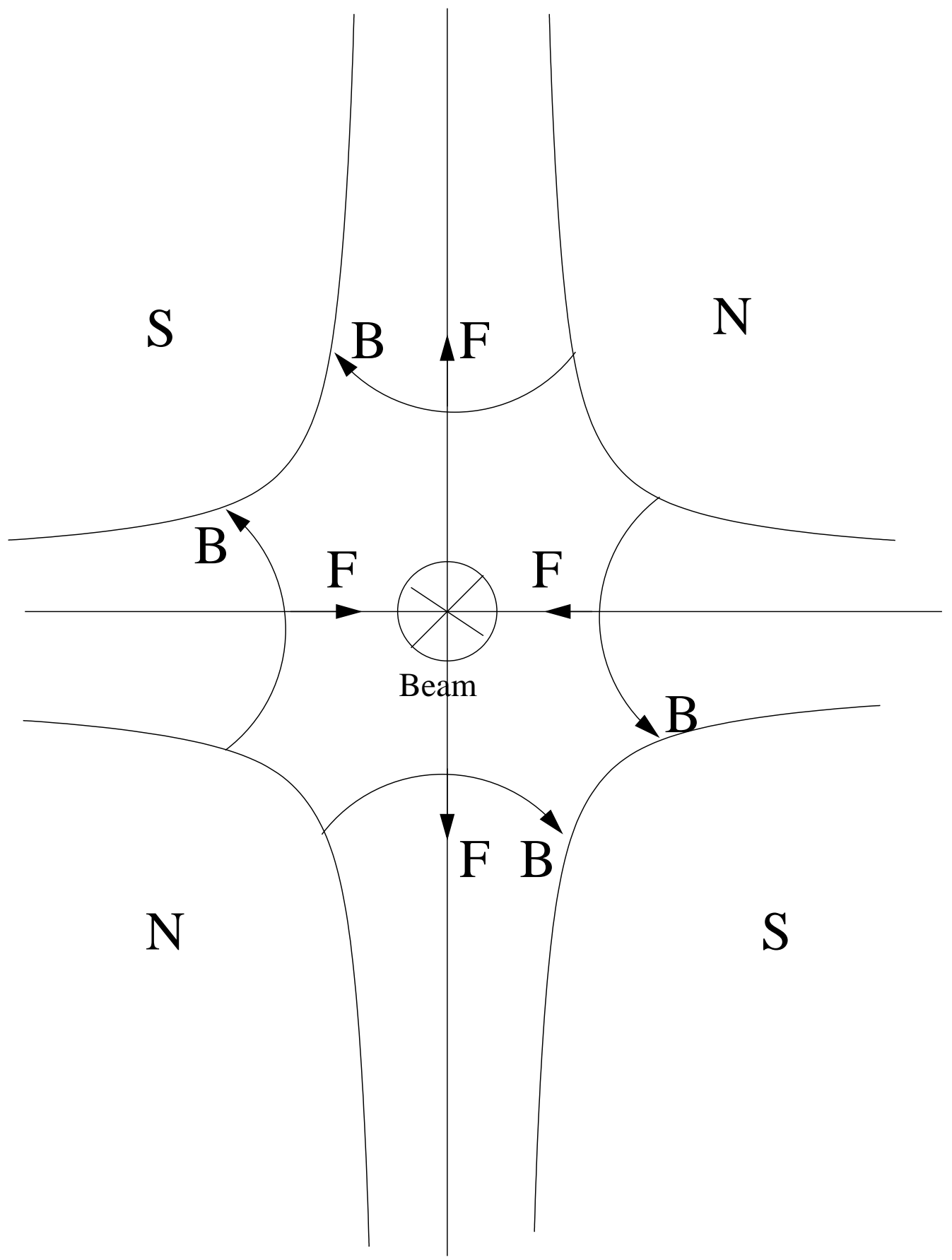

Figure 1.9: The direction of the magnetic forces in 4 different points of a quadrupole magnetic field. 


$$
\Delta y^{\prime}=\frac{\Delta l}{\rho}
$$

where the thin lens approximation has been used. The sign difference in the two expressions is a consequence of the direction of the magnetic forces in a quadrupole as can be seen in Fig. 1.9. Fig. 1.9 shows that while a particle in a positive horizontal position experiences a kick in the $-x$ direction a particle in a positive vertical position experiences a kick in the $+y$ direction.

Eq. 1.45 together with Eq. 1.10 lead to:

$$
\begin{aligned}
\Delta x^{\prime} & =-\frac{q B_{y} \Delta l}{p} \\
\Delta y^{\prime} & =\frac{q B_{x} \Delta l}{p}
\end{aligned}
$$

where we have used the condition of equilibrium for a particle with charge $e$ moving through an arc of radius $\rho$ with momentum $p$ in a magnetic field.

The components of a magnetic field of a perfect aligned quadrupole are given by:

$$
\begin{aligned}
& B_{x}=B^{\prime} y \\
& B_{y}=B^{\prime} x
\end{aligned}
$$

If the quadrupole is rotated about a longitudinal axis by an angle $\phi$ the new expressions of the magnetic field are given by:

$$
B_{x}=B^{\prime}(y \cos (2 \phi)-x \sin (2 \phi))
$$




$$
B_{y}=B^{\prime}(x \cos (2 \phi)+y \sin (2 \phi))
$$

Using the definition for magnetic rigidity in Eq. 1.10 and Eq. 1.49:

$$
\begin{aligned}
& \Delta x^{\prime}=-\frac{B_{y} \Delta l}{B \rho}=-\frac{B^{\prime} \Delta l}{B \rho}(x \cos (2 \phi)+y \sin (2 \phi)) \\
& \Delta y^{\prime}=\frac{B_{x} \Delta l}{B \rho}=\frac{B^{\prime} \Delta l}{B \rho}(y \cos (2 \phi)-x \sin (2 \phi))
\end{aligned}
$$

Under the assumption that $\phi$ is a small angle the following relations can be obtained :

$$
\begin{aligned}
\Delta x^{\prime} & =-\frac{B^{\prime} \Delta l}{B \rho}(x+2 \phi y) \\
\Delta y^{\prime} & =\frac{B^{\prime} \Delta l}{B \rho}(y-2 \phi x)
\end{aligned}
$$

This Equation give the total magnetic kick that a particle experiences when it goes through the quadrupole. Such magnetic kick has two major sources: the gradient of the quadrupole itself and the induced kick from the opposite plane due to the roll of the quadrupole. The gradient of the quadrupole can be divided between a design gradient, $\frac{B_{0}^{\prime} \Delta l}{B \rho}$, and a gradient error, $\frac{B_{e r r}^{\prime} \Delta l}{B \rho}$. It is then possible to write:

$$
\begin{aligned}
\Delta x^{\prime} & =-\left(\frac{B_{0}^{\prime} \Delta l}{B \rho}+\frac{B_{e r r}^{\prime} \Delta l}{B \rho}\right)(x+2 \phi y) \\
& \cong-\left(\frac{B_{0}^{\prime} \Delta l}{B \rho}+\frac{B_{e r r}^{\prime} \Delta l}{B \rho}\right) x-\frac{B_{0}^{\prime} \Delta l}{B \rho} 2 \phi y
\end{aligned}
$$




$$
\begin{aligned}
& =\Delta x_{0}^{\prime}-\frac{B_{e r r}^{\prime} \Delta l}{B \rho} x-\frac{B_{0}^{\prime} \Delta l}{B \rho} 2 \phi y \\
& =\Delta x_{0}^{\prime}-B_{1} x+A_{1} y
\end{aligned}
$$

and similarly,

$$
\begin{aligned}
\Delta y^{\prime} & \cong \Delta y_{0}^{\prime}+\frac{B_{e r r}^{\prime} \Delta l}{B \rho} y-\frac{B_{0}^{\prime} \Delta l}{B \rho} 2 \phi x \\
& =\Delta y_{0}^{\prime}+B_{1} y+A_{1} x
\end{aligned}
$$

where

$$
\begin{aligned}
\Delta x_{0}^{\prime} & =-\frac{B_{0}^{\prime} \Delta l}{B \rho} x \\
\Delta y_{0}^{\prime} & =\frac{B_{0}^{\prime} \Delta l}{B \rho} y \\
A_{1} & =-\frac{2 B^{\prime} \Delta s \phi}{B \rho} \\
B_{1} & =\frac{B_{e r r}^{\prime} \Delta s}{B \rho}
\end{aligned}
$$

Terms of second order are neglected in Eq. 1.51 and Eq. 1.52.

Error analysis in this work involved the determination of $\Delta x^{\prime}-\Delta x_{0}^{\prime}$ and $\Delta y^{\prime}-\Delta y_{0}^{\prime}$ which we will call simply $\Delta x^{\prime}$ and $\Delta y^{\prime}$. With these new definitions Eq. 1.51 and Eq. 1.52 can be rewritten as:

$$
\begin{aligned}
& \Delta x^{\prime}=-B_{1} x+A_{1} y \\
& \Delta y^{\prime}=B_{1} y+A_{1} x
\end{aligned}
$$




\subsubsection{Coupling from Solenoidal Fields}

Not all sources of linear coupling are included in Eq. 1.56. It is also possible to obtain coupling through the variables $y^{\prime}$ and $x^{\prime}$ when axial magnetic fields are present. The mathematical form of such terms can be easily obtained from the Lorentz force (see for example [26]) and is given by:

$$
\Delta x_{s}^{\prime}=\frac{\Delta L B_{s} y^{\prime}}{B \rho}
$$

where $\Delta x_{s}^{\prime}$ is the horizontal kick that the orbit experiences due to the axial magnetic field. I will evaluate the ratio between the terms that account for the axial magnetic field and the skew quadrupole.

$$
\frac{\Delta x_{s}^{\prime}}{\Delta x_{\text {skew }}}=\frac{\Delta L B s x^{\prime}}{B \rho A_{1} x}
$$

where $\Delta x_{\text {skew }}$ is the kick due to the skew quadrupole alone.The maximum values of $x$ and $x^{\prime}$ are given by [30]:

$$
\begin{aligned}
& x_{\max }=\sqrt{\frac{\epsilon \beta}{\pi}} \\
& x_{\max }^{\prime}=\sqrt{\frac{\epsilon \gamma}{\pi}}
\end{aligned}
$$

Replacing Eq. 1.59 in Eq. 1.58 we get:

$$
\frac{\Delta x_{s}^{\prime}}{\Delta x_{\text {skew }}}=\frac{\Delta L B_{s} \sqrt{\gamma}}{B \rho \sqrt{\beta}}
$$

The main source of axial magnetic fields in RHIC are the solenoids used in the

experiments like PHENIX. Using typical values of the PHENIX experiment 


\begin{tabular}{|c|c|c|c|}
\hline Description & Symbol & Value (injection) & Value (storage) \\
\hline Magnetic Rigidity & $|B \rho|$ & $97.5 \mathrm{~T}-\mathrm{m}$ & $839.5 \mathrm{~T}-\mathrm{m}$ \\
Beta at Phenix & $\beta$ & $4.8 \mathrm{~m}$ & $3.1 \mathrm{~m}$ \\
Gamma at Phenix & $\gamma$ & $0.32 \mathrm{~m}(-1 / 2)$ & $0.32 \mathrm{~m}(-1 / 2)$ \\
Solenoid Lenght & $\Delta L$ & $1 \mathrm{~m}$ & $1 \mathrm{~m}$ \\
Axial Field & $B_{s}$ & 0.5 Tesla & $0.5 \mathrm{Tesla}$ \\
\hline
\end{tabular}

Table 1.1: Phenix solenoids parameters. Some of the parameters were taken from $[31]$

(Table 1.1), Eq. 1.60 evaluated at injection is 1.31 and the same equation evaluated at storage is 0.2 . The axial magnetic fields produced by the experiment might then have a significant effect in the linear coupling of the machine, at least for injection. During accelerator physics beam experiments and machine development is then neccesary to make sure that these magnets are off.

\subsubsection{Global Coupling: $\Delta q_{\min }$}

This dissertation is mainly concerned with local error evaluation and correction. However, there are global measurements that are used in this thesis to test the effectiveness of the local correction. One of those quantities is

the $\Delta q_{\text {min }}$, the minimum possible separation between the horizontal and the vertical plane.

$\Delta q_{\min }$ can be found after solving the system of differential equations (see Eq. 1.32):

$$
x^{\prime \prime}+K(s) x=-\frac{B_{y}(s)}{B \rho}
$$




$$
=\frac{\Delta x^{\prime}}{\Delta l}
$$

and similarly for the y plane:

$$
\begin{aligned}
y^{\prime \prime}+K(s) y & =\frac{B_{x}(s)}{B \rho} \\
& =\frac{\Delta y^{\prime}}{\Delta l}
\end{aligned}
$$

Therefore the equations of motion become:

$$
\begin{aligned}
x^{\prime \prime}+K_{x}(s) x & =-\frac{B^{\prime}}{B \rho}(x+2 \phi y) \\
y^{\prime \prime}+K_{y}(s) y & =\frac{B^{\prime}}{B \rho}(y-2 \phi x)
\end{aligned}
$$

The general solution for these equation can be seen for example in [23]. Those solutions are 2 eigen-modes in terms of which any particle motion can be described. The 2 eigenfrequencies of the eigen-modes are([24], [29]):

$$
q_{I, I I}=\frac{1}{2}\left(q_{x}+q_{y} \pm \sqrt{(q x-q y)^{2}+\Delta q_{\min }^{2}}\right)
$$

where $q_{x}$ and $q_{y}$ are the fractional tunes which one would expect without coupling, $q_{I, I I}$ are the couple tunes, and $\Delta q_{\min }$ is a quantity that is directly related with the coupling errors throughout the machine (see for example [24], [25], [27]). $\Delta q_{\min }$ can be measured by varying any of the tunes the tunes $q_{x}$ or $q_{y}$ while measuring the real tunes of the machine in both planes like in Fig. 1.10. The closest distance between $q_{I}$ and $q_{I I}$ graphs is equal to $\Delta q_{\min }$ as can be verified from Eq. 1.64 . 


\section{Coupled Tunes vs X Tune Knob}

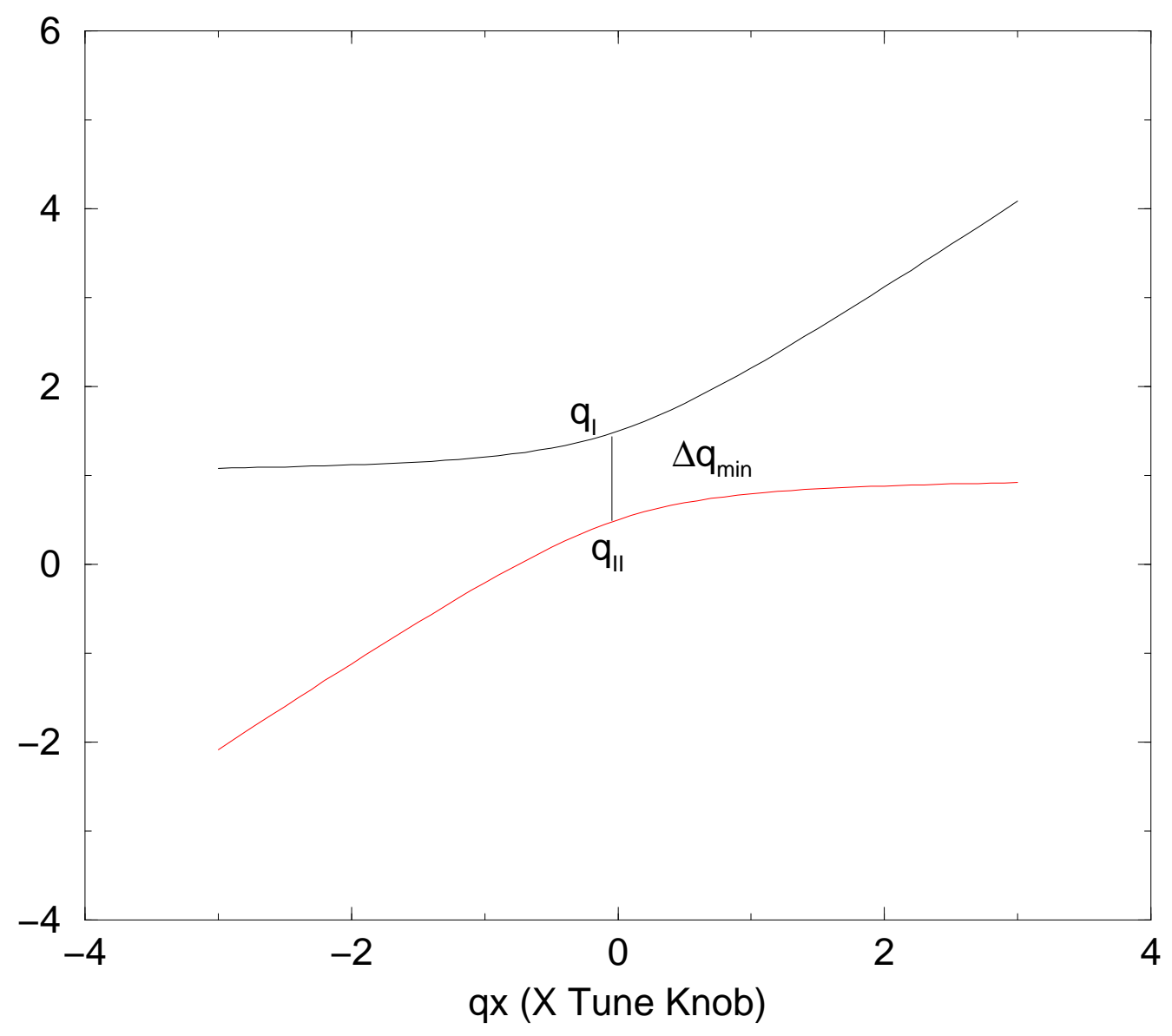

Figure 1.10: Measurement of $\Delta q_{\min }$ from coupled tunes. 
$\Delta q_{\text {min }}$ is a very important quantity used on a routine basis to determine the global coupling of an accelerator and it will be used here to evaluate the effectiveness of the local decoupling performed with the techniques developed in this thesis. 


\section{Chapter 2}

\section{Action and Phase Jump Analysis}

Optics errors in an accelerator lattice can be studied systematically by well known techniques like the Measured Response Matrix [4]. In this technique the strengths of all dipole correctors in the ring are varied while registering the change in the orbit data. This information can be used afterwards to accurately calibrate the linear optics in electron storage rings.

There are other techniques like the Model Independent Analysis (MIA) [5] that were initially applied in LINACS and recently this technique has been extended to storage rings [6].

In MIA analysis, the particle centroid motion is described in terms of temporal and spatial patterns. The BPM data of several pulses from a LINAC or turn by turn trajectories from a ring are then used to create a matrix and after Singular Value Decomposition Analysis [7] the parameters affecting the

beam motion can be determined. One of the advantages of this technique 
is that doesn't require a beam line model and is non-invasive or minimally invasive to accelerator operations.

During the early stage of an accelerator commissioning, methods that provide information about errors at one specific location of the ring are preferred. One of the most common techniques of this kind is the closed orbit bump. A closed orbit bump is generally created with 3 dipole correctors set to values such that the orbit is perturbed only in a localized region of the accelerator. More than 3 dipole correctors are also used to gain control over parameters like the maximum position of the bump and the angle respect to the azimuthal coordinate $s$.

Closed orbits bumps can be used to find certain kind of lattice errors (see Appendix D). During the 2000 and 2001 RHIC commissioning, closed orbit bumps were made around some of the triplets in the Interactions Regions of the accelerator rings, leading to reliable values of the skew quadrupole errors [16]. The technique also give estimates of the triplet integrated gradient error.

Closed orbit bumps in combination with tune measurements are also a valuable tool to evaluate nonlinear errors. This procedure was used in the RHIC 2001 run[17] and the RHIC 2003 run to correct non linearities at the RHIC IR's. For a complete reference of the methods that have been used or methods that are under current development to measure non linearities see [18].

Beam based alignment techniques are also widely used to measure quadrupole error alignments ( see [8] and [9]). The "classical" beam based alignment 
technique is to vary the strength of one or several quadrupoles and measure the downstream deflection. This technique is limited by the systematic error caused by the shift of quadrupole center when its strength is varied.

There is still another technique to localize and measure errors based on the fact that regions bounded by magnetic errors can be modeled with the design beta functions even though the real beta functions might change (see Section 1.5). The general idea is to fit certain sectors of the orbit to the designed model and systematically increase the extension of the sector until the fit start to fail. The place where the fit starts to fail is a potential place where a magnetic error might be present. This technique has been used successfully in the past on closed orbits ( see for example [13] and [14]) and most recently in the PEP-II commissioning [15]) .

This approach, however, is not the most common approach to error analysis in an accelerator lattice. One of the reasons is that in its original form this technique requires many iterations just to find the location of a particular error.

The Action and Phase analysis that is described in this chapter was born as a simple tool to find gross optics errors in RHIC but it has evolved into a technique that is able to give precise error estimates of a group of quadrupole magnets such as a triplet. This information is enough, for example, to set local correctors in order to correct for the linear magnetic errors without realigning the magnets as it will be explained below. This method also allowed the development of a technique to find nonlinear errors in the interaction regions. 
Since orbits for action and phase analysis are produced with dipole correctors, action and phase analysis is free from systematic errors like the ones introduced by the quadrupoles in the beam based alignment techniques mentioned previously.

Another advantage of the action and phase analysis method is the ability to combine the strength of techniques to find local errors (like the orbit bump method) and the strength of general methods that allow the determination of many errors with the same set of orbits.

In this Chapter I will describe the basic idea of action and phase analysis and the first evidences that lead to linear and non linear error determination at RHIC IR's.

\subsection{The Method}

Under ideal conditions, the action and phase of the betatron oscillations of a particle should remain constant throughout the machine. Magnetic errors in the different elements of the ring can lead to a change of these two constants of motion. These changes can indeed be used to determine the location of such errors and their strengths.

It is then necessary to have measurements of action $J$ and phase $\psi$ in many places in the ring as possible. The Beam Position Monitors are the natural choice to indirectly measure $J$ and $\psi$ all around the ring. This is done 
by taking pairs of adjacent BPM's and inverting the equations:

$$
\begin{aligned}
& x_{1}=\sqrt{2 J \beta_{1}} \sin \left(\psi_{1}-\psi\right) \\
& x_{2}=\sqrt{2 J \beta_{2}} \sin \left(\psi_{2}-\psi\right)
\end{aligned}
$$

to obtain:

$$
\begin{aligned}
J & =\frac{\left(x_{1}^{2}+x_{2}^{2}-2 x_{1} x_{2} \cos \left(\psi_{1}-\psi_{2}\right)\right)}{\sin ^{4}\left(\psi_{2}-\psi_{1}\right)} \\
\tan \psi & =\frac{x_{1} \sin \psi_{2}-x_{2} \sin \psi_{1}}{x_{1} \cos \psi_{2}-x_{2} \cos \psi_{1}}
\end{aligned}
$$

where $x_{1}$ and $x_{2}$ correspond to any two adjacent BPM measurement, $\beta_{1}, \beta_{2}, \psi_{1}$ and $\psi_{2}$ are their corresponding beta functions and phase advances and finally $J$ and $\psi$ are the action and phase.

Eq. 2.2 is applied to all adjacent BPM pair measurements in the ring to obtain functions of action and phase with respect to $s$.

\subsection{Application of Action and Phase analysis to RHIC 2000 First turn Orbits}

In order to apply the algorithm defined by Eq. 2.2 to RHIC BPM measurements it is necessary to know the design beta functions and phase advances at all BPM's in the ring. The MAD program [10] is used in RHIC to numerically

find the beta functions and phase advances defined by Eq. 1.16. The solution 
given by MAD for the beta functions corresponds to an ideal lattice and it is a solution that closes on itself for analysis of closed orbits.

A software application (MODULAR_IR2) was created to read the BPM orbit data, the Twiss files and other auxiliary files ( see Chapter 9 ) and to repeatedly apply Eq. 2.2 and create graphs of $J$ and $\psi$ as function of the axial coordinate $s$.

In order to test the early versions of MODULAR_IR2, a small program that generates single pass orbits (BMODEL for Blue ring and YMODEL for Yellow ring) from the betatron equation (Eq. 1.17) was created. This simple program allows the user to choose the amplitude of the betatron oscillation or, equivalently, the action $J$ and the phase advance $\delta$. The first simulation was done by setting a single dipole corrector bo7-th12 such that the downstream action of the betatron oscillation was $2000 \mathrm{~nm}$. Fig. 2.1 is obtained after processing the previous simulated single pass orbits with MODULAR_IR2. Action and phase remain constant except at one point that corresponds to the location of the dipole corrector bo7-th12. The values of action and phase in the graph downstream of the dipole corrector exactly correspond to the values used in the program BMODEL to simulate the orbit. The third graph of Fig. 2.1 is a representation of RHIC lattice. The thin long bars represent quadrupole magnets while the long short bars in between represent dipole magnets. The regions limited for big empty spaces are known as the Interaction Regions (IR's).

The real trajectories to be analyzed were produced by turning on a dipole 


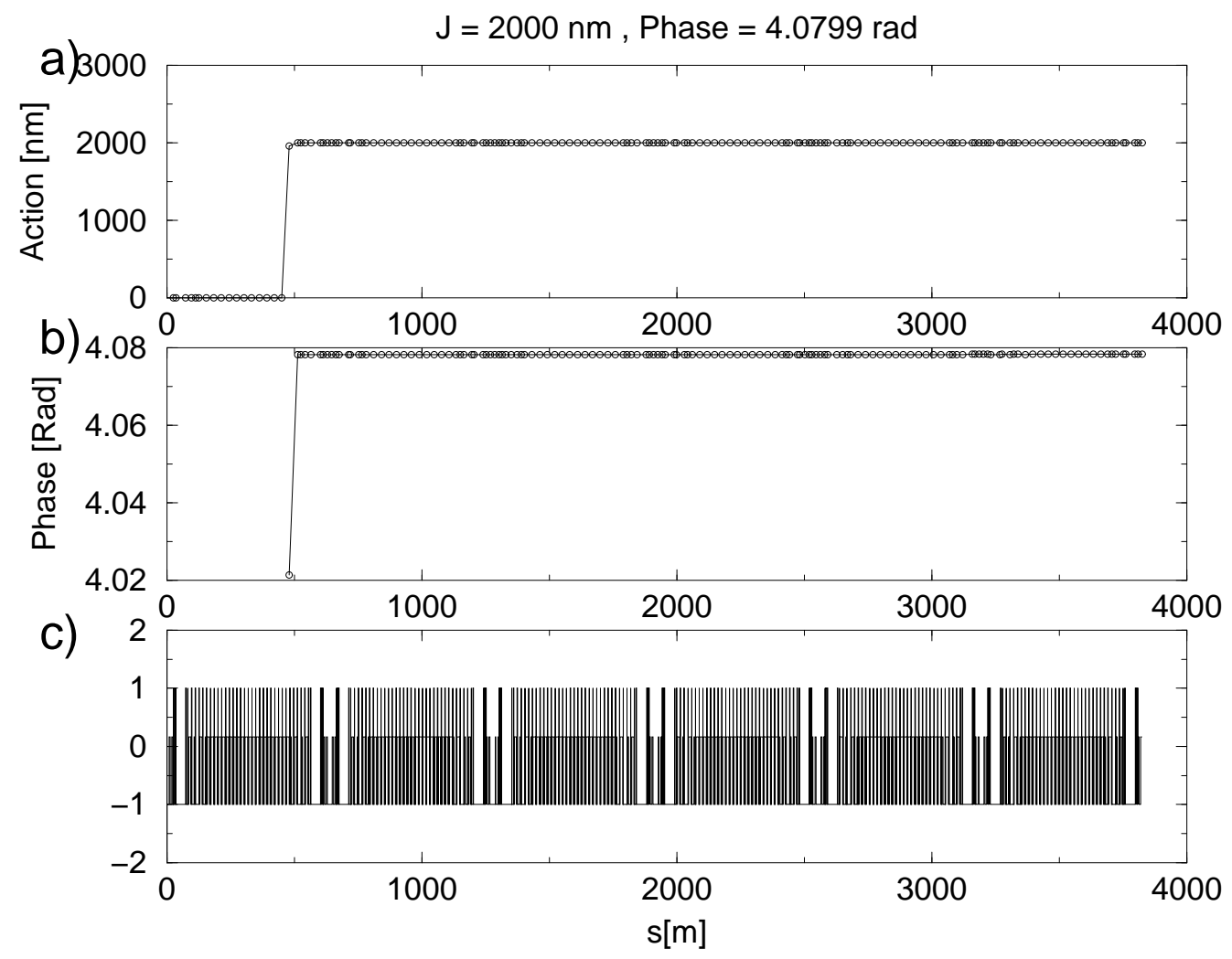

Figure 2.1: a) Action analysis of an orbit calculated with the betatron equation. b) Phase analysis of the same orbit. c) Representation of the RHIC lattice. The long bars represent quadrupoles while the short ones represent dipoles. 
corrector and then making the difference trajectory with the closest single trajectory in time available with the corrector off (baseline). The strength of the dipole corrector is chosen such that the betatron oscillations are as big as possible to get a strong orbit signal while avoiding beam losses as much as possible. Angles of a few tenths of mRad are an adequate value in the RHIC case giving first turn trajectories as large as $10 \mathrm{~mm}$ or more in the arcs. The difference trajectories are helpful, first to eliminate any possible dipole kick present in the ring except the one that excites the oscillation and second to eliminate any possible systematic error in the BPM measurements.

Fig 2.2 and Fig. 2.3 show the action and phase analysis of an orbit excited with a vertical dipole corrector (bo7-tv7 in RHIC nomenclature) located at $s=528.2 \mathrm{~m}$. It is possible to identify in these graphs regions were the action and phase remains roughly constant, while they make significant jumps at the IR's.

The possible source of these jumps can be either gradient errors, skew quadrupole errors, or any other multipole component of the IR quadrupole magnets (triplets). Dipole errors cannot be the source of these jumps because the difference orbit eliminates any possible dipole error present in the orbit except for the one that is exciting the orbit. Some of the first turn difference orbits taken during RHIC 2000 run indicate the presence of significant linear coupling at the IR's.Take for example the orbit shown in Fig. 2.4. This orbit was excited with a horizontal dipole corrector at $s=1672.9 \mathrm{~m}$. As the betatron oscillation goes through the first IR between $s=603 \mathrm{~m}$ and $s=675 \mathrm{~m}$, a 

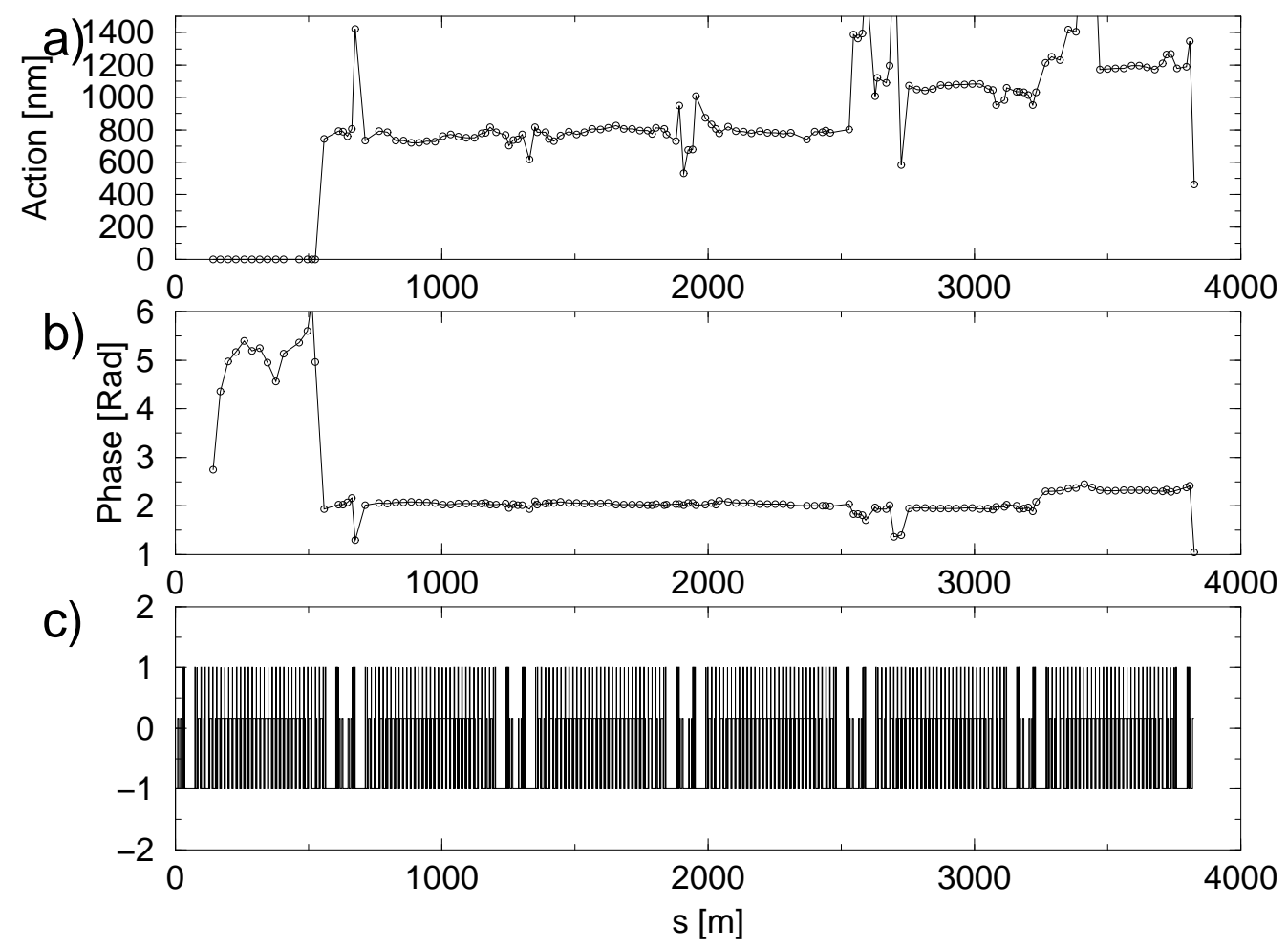

Figure 2.2: a) Action analysis in the vertical plane of a difference trajectory obtained during the 2000 RHIC run. The oscillations were excited by a the dipole corrector bo7-tv7 located at $s=528.2 \mathrm{~m}$. b) Phase analysis of the same orbit. c) Lattice representation of RHIC. 


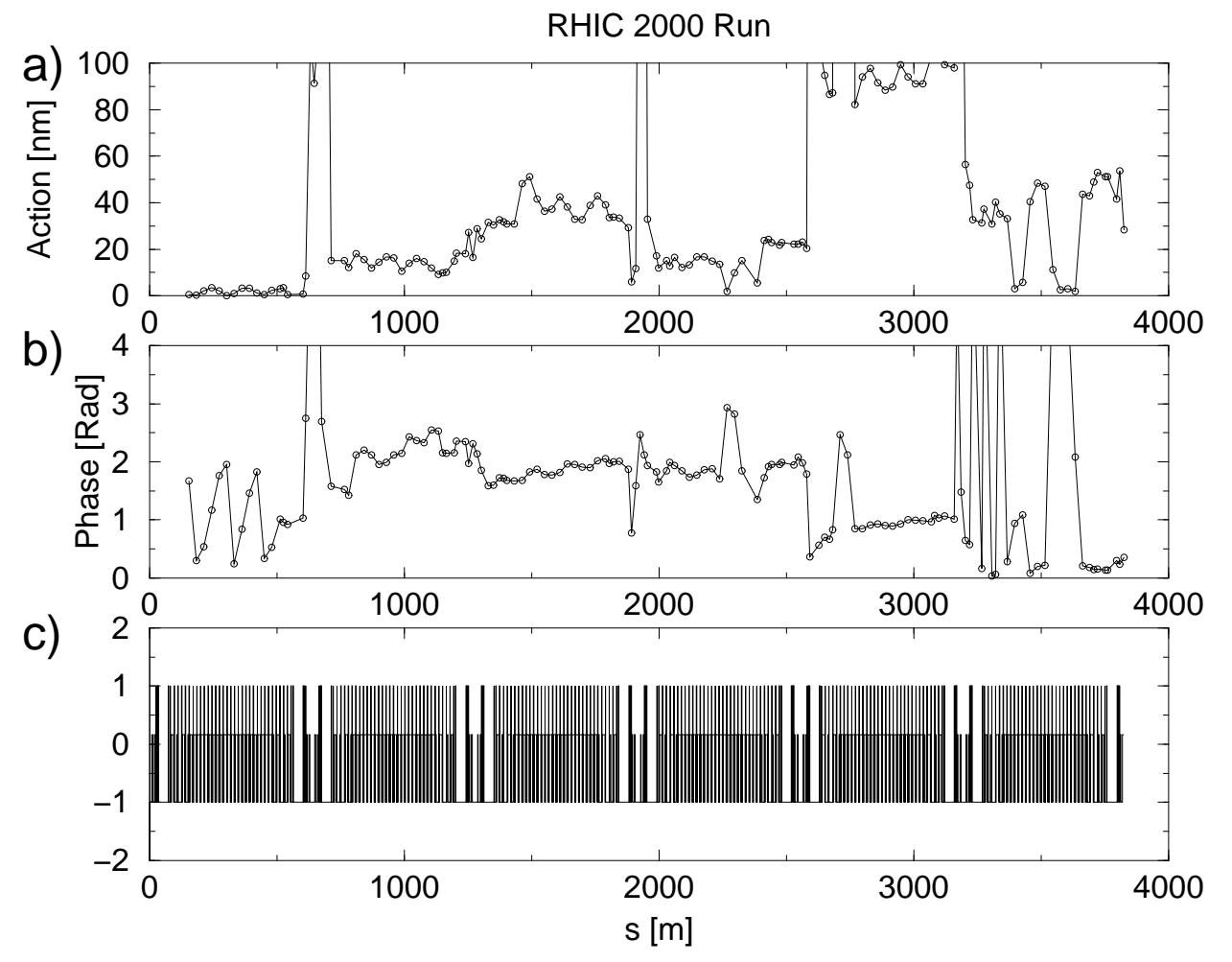

Figure 2.3: a) Action analysis in the horizontal plane of a difference trajectory obtained during the 2000 RHIC run. In this case, the oscillations are induced from the vertical plane due to strong coupling at the IRs. b) Phase analysis of the same orbit. c) Lattice representation of RHIC. 

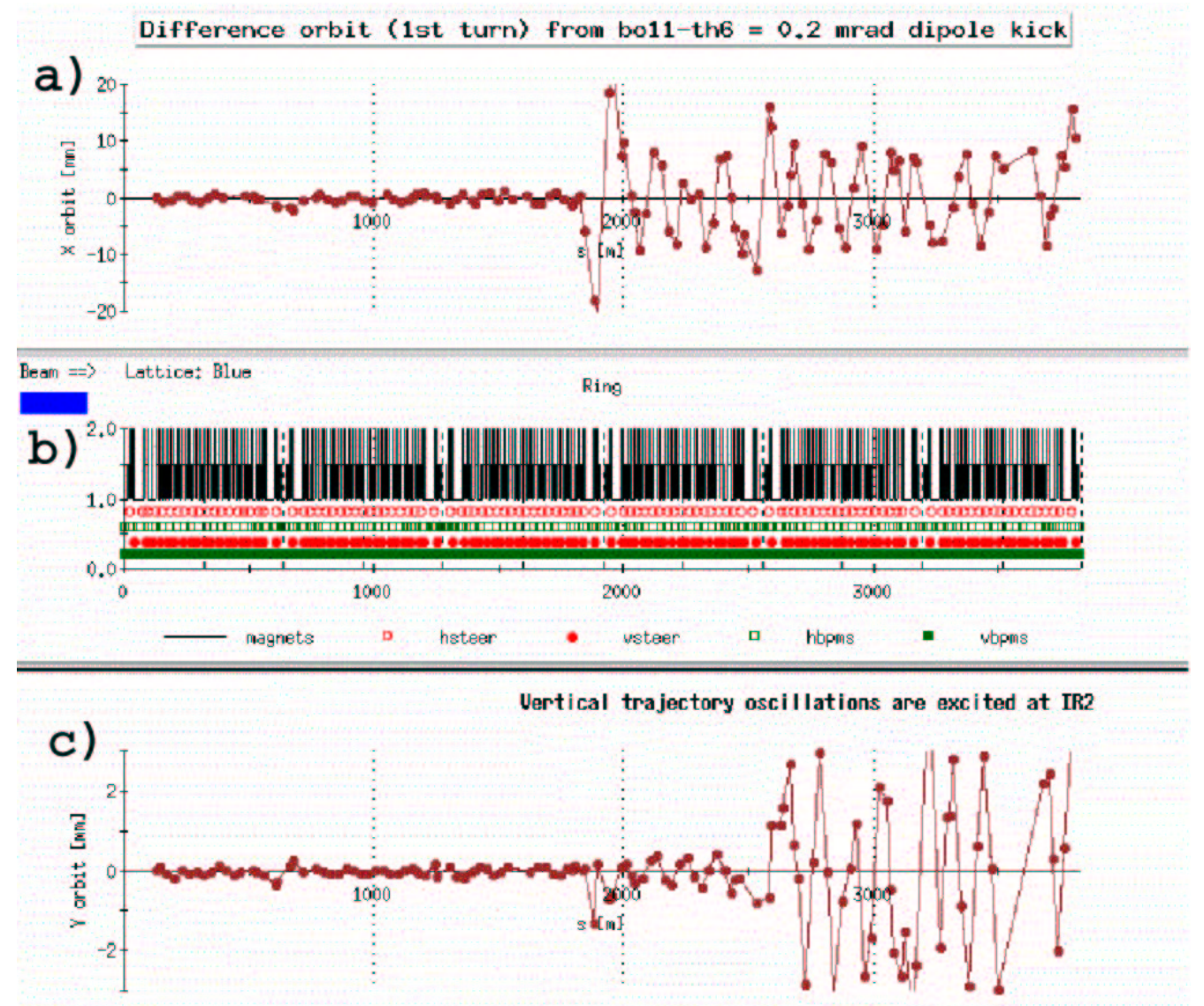

Figure 2.4: principal window of the RHIC orbit display program. a) First turn orbit excited just before IR12. b) Lattice representation of RHIC. c) Induced orbit in the vertical plane due to coupling at IR's. 
betatron oscillation is induced in the opposite plane. This can be clearly seen for the change in the amplitude of the orbit in the vertical plane. These are clear signs of local coupling sources in the machine, and since the induced amplitude grew proportionally with the amplitude of the excited orbit it was possible to conclude that the coupling was linear. 


\section{Chapter 3}

\section{Linear Errors at RHIC's IRs and First Turn Trajectory Analysis}

The most significant sources of optics errors during the RHIC 2000 run were located at the interaction regions as was shown in Chapter 2. Those errors turned out to be linear errors, i.e. gradient error and skew quadrupole errors. The procedure to quantify such errors from the action and phase analysis of first turn difference trajectories is discussed in this chapter.

\subsection{Determination of Skew Quadrupole Errors from Action and Phase Graphs}

The graphs of action and phase shown in Fig. 2.2 and Fig. 2.3 suggest that the orbit can be modeled with an independent betatron oscillation in each arc. This fact is also supported by the theoretical results obtained in Section 1.5. For each arc the difference lies in the $\psi$ and $J$ that best fit the orbit. Action 
and phases between adjacent arcs can be used to estimate the skew quadrupole errors at the interaction regions. The formulas to be employed are derived in the following lines.

Assume there is a magnetic error $\Delta x^{\prime}$ at some position $s_{0}$ inside a specific IR. The orbit in the adjacent arc to the IR under study such that $s<s_{0}$ can be represented by:

$$
x_{L}(s)=\sqrt{2 J_{L} \beta_{x}(s)} \sin \left(\psi_{x}(s)-\psi_{L}\right)
$$

and the orbit in the adjacent arc to the IR under study such that $s>s_{0}$ will be:

$$
\begin{aligned}
x_{R}(s) & =\sqrt{2 J_{R} \beta_{x}(s)} \sin \left(\psi_{x}(s)-\psi_{R}\right) \\
& =x_{L}(s)+\Delta x^{\prime} \sqrt{\beta\left(s_{0}\right) \beta_{x}(s)} \sin \left(\psi_{x}(s)-\psi\left(s_{0}\right)\right)
\end{aligned}
$$

where the the subscripts $L$ and $R$ were used to label quantities evaluated at $s<s_{0}$ and at $s>s_{0}$ respectively.

Since $\sin \left(\psi_{x}(s)\right)$ and $\cos \left(\psi_{x}(s)\right)$ are orthogonal functions the following equations can be derived from Eq. 3.2:

$$
\begin{gathered}
\Delta x^{\prime} \sqrt{\beta\left(s_{0}\right)} \cos \left(\psi\left(s_{0}\right)\right)=\sqrt{2 J_{R}} \cos \left(\psi_{R}\right)-\sqrt{2 J_{L}} \cos \left(\psi_{L}\right) \\
\Delta x^{\prime} \sqrt{\beta\left(s_{0}\right)} \sin \left(\psi\left(s_{0}\right)=\sqrt{2 J_{R}} \sin \left(\psi_{R}\right)-\sqrt{2 J_{L}} \sin \left(\psi_{L}\right)\right.
\end{gathered}
$$


Squaring and adding these two equations leads to:

$$
\Delta x^{\prime}=\sqrt{\frac{2 J_{R}+2 J_{L}-4 * \sqrt{J_{R}} \sqrt{J_{L}} \cos \left(\psi_{R}-\psi_{L}\right)}{\beta\left(s_{0}\right)}}
$$

and if Eq. 3.3 are divided we get:

$$
\tan \psi\left(s_{0}\right)=\frac{\sqrt{2 J_{R}} \sin \left(\psi_{R}\right)-\sqrt{2 J_{L}} \sin \left(\psi_{L}\right)}{\sqrt{2 J_{R}} \cos \left(\psi_{R}\right)-\sqrt{2 J_{L}} \cos \left(\psi_{L}\right)}
$$

Eq. 3.4 is a very important result. It relates the strength of the magnetic error with quantities that can be determined experimentally. Such quantities ( $J_{L}$, $J_{R}, \psi_{L}$ and $\left.\psi_{R}\right)$ can be extracted from plots of action and phase like the one shown in Fig. 3.1. Since magnetic errors in the arcs are small when compared with the interaction region errors, phase and action are assumed constant within the arcs. It is then easy to do a rough estimate of all required actions and phases by just drawing horizontal lines over the distributions of actions and phases within each arc ${ }^{1}$. Fig. 3.1 shows how each variable is obtained from the graph to estimate $\Delta x^{\prime}$ at IR12. $\Delta x^{\prime}$ is a quantity that contains information about the strength of the magnetic error at the particular place of study in the ring. RHIC has a local correction system to suppress these errors in each triplet of all interaction regions. In the following section, $\Delta x^{\prime}$ will be related with the strengths needed in the RHIC local correction system in order to suppress the skew quadrupole errors.

\footnotetext{
${ }^{1}$ More rigorous algorithms were developed to find action and phases in the arc and they will be discussed in Appendix C
} 


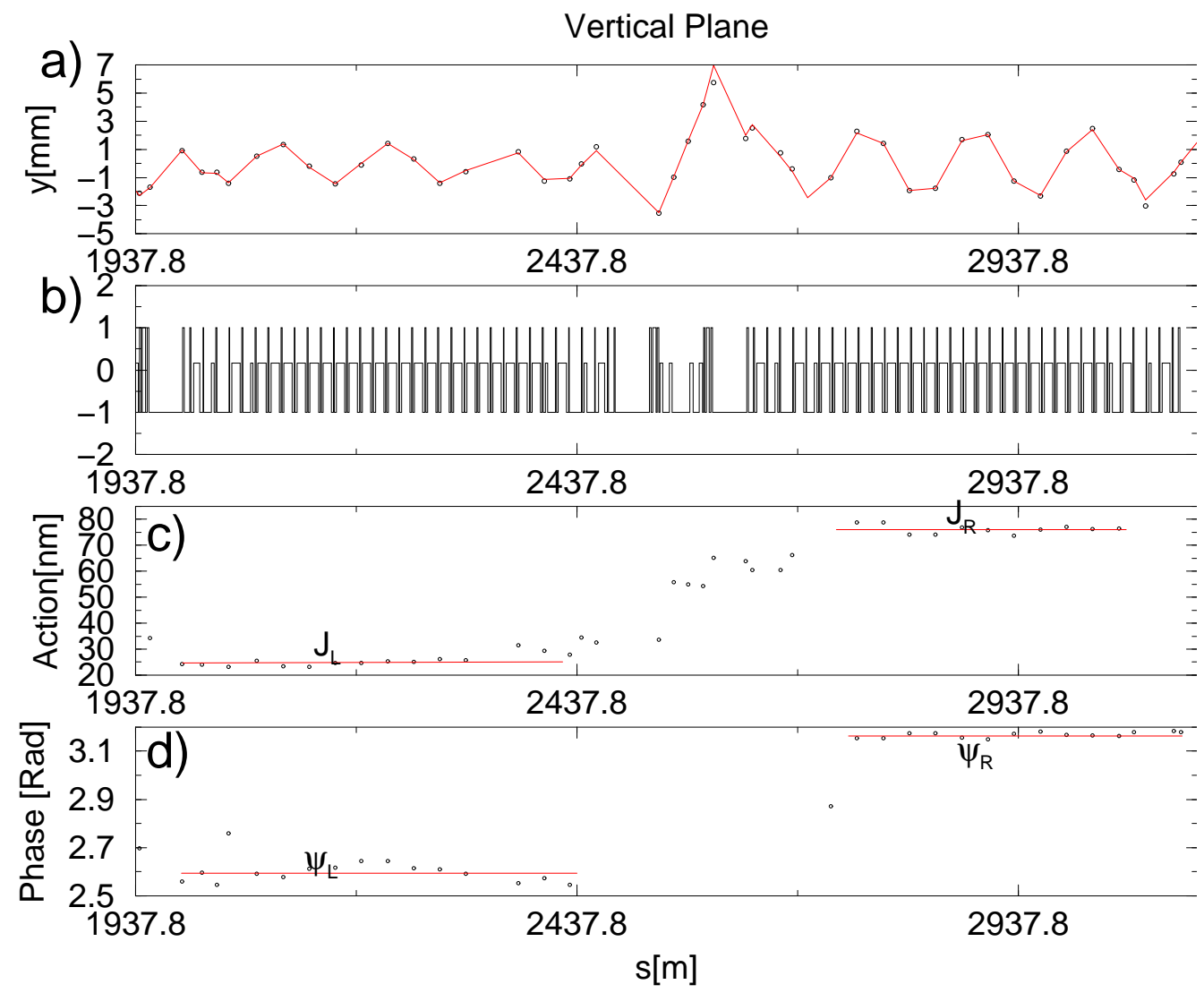

Figure 3.1: a) Measured orbit (small circles) in the vertical plane fitted to the model (solid line) arc by arc. The action and phase of the model is obtained by averaging action and phases within each arc (see subroutine fitted $_{p}$ os in Appendix C). b) Lattice representation of RHIC. c) Action analysis of the measured orbit. d) Phase analysis of the measured orbit. 


\subsection{RHIC Local Linear Coupling Correction System}

Since the beta functions at RHIC IRs can be many times bigger than in any other place in the ring (see Fig. 3.2), IRs are specially sensitive to magnet errors, particularly roll errors. Studies performed during the RHIC design stage show that rolls of the order of mrad can cause tune shifts of about 0.1 units, big enough to lead the beam to an undesirable resonance condition [11]. In order to correct for these effects, each RHIC IR was provided with 2 skew quadrupole corrector packages, one for each triplet of the corresponding IR (see [12]).

In order to understand how the corrector works, it is convenient to write the total orbit $x_{\text {total }}$ produced by the skew error in the triplet and the corrector:

$$
x_{\text {total }}(s)=\left[\sqrt{2 J_{x}^{\text {Trip }}}-s k_{r e} y^{s c} \sqrt{\beta_{x}^{s c}}\right] \sqrt{\beta_{x}(s)} \sin \left(\psi_{x}(s)-\psi_{x}^{\text {Trip }}\right)
$$

where $J_{x}^{\text {Trip }}=\left(\Delta x^{\prime}\right)^{2} \frac{\beta\left(s_{0}\right)}{2}, y^{s c}$ is the beam position in the plane of the kick (in this case the vertical plane) at the location of the skew quadrupole corrector, $\beta_{x}^{s c}$ is the beta function at the position of the skew quadrupole corrector, $s k_{r e}$ is the strength of the corrector in numbers that can be understood by the Ramp Editor Manager (see Chapter. F), $\psi_{x}^{\text {Trip }}$ the phase at the triplet and $\beta_{x}(s)$ and $\psi_{x}(s)$ the beta functions and phase functions. The strength of the corrector must be adjusted such that the oscillation due to the skew error is suppressed or in mathematical terms $x_{\text {total }}=0$. Using this condition in Eq. 3.6 


\section{Horizontal Beta Functions}

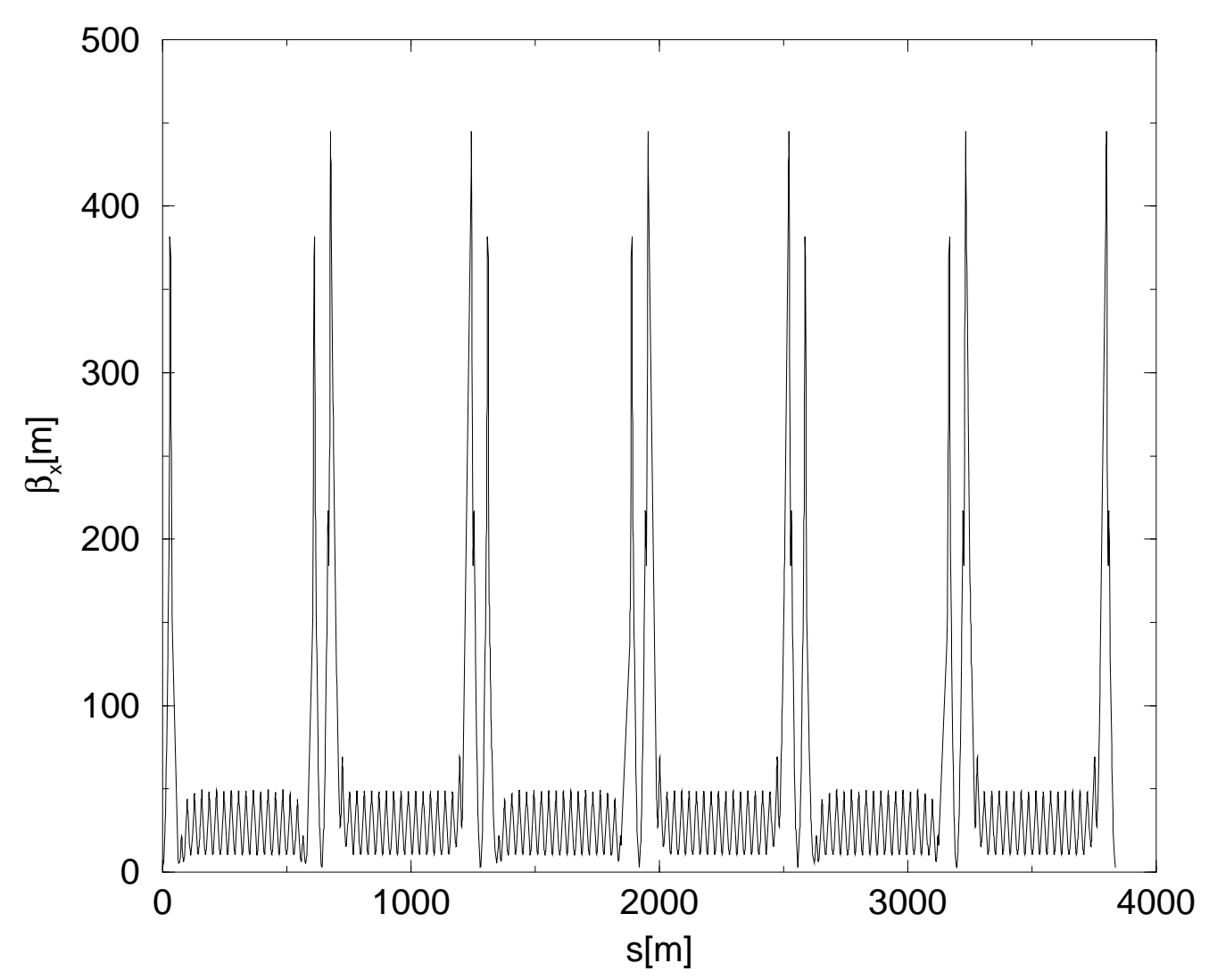

Figure 3.2: Horizontal beta functions used during the polarized proton RHIC run (Nov 302001 to Jan 23 2002). Beta functions are significantly bigger at the interaction regions than in any other place of the ring. 
the strength needed in the corrector is:

$$
s k_{r e}=\frac{\sqrt{2 J_{x}^{\text {Trip }}}}{y^{s c} \sqrt{\beta_{x}^{s c}}}
$$

Since the two skew quadrupole correctors of a specific IR have a phase advance close to $\pi$, it is possible to use a single skew quadrupole corrector to compensate the skew errors in the whole IR. This is specially useful when it is not possible to have individual estimates of $J_{x}^{\text {Trip }}$ for the left and right triplets, but rather an integrated value $J_{x}^{I R}$. In that case the optimal value of the corrector is given

by Eq. 3.7 when $J_{x}^{\text {Trip }}$ is replaced by $J_{x}^{I R}$, the equivalent action associated with the overall magnetic kick due to skew errors that the orbit receive at the IR.

Notice that $J_{x}^{\text {Trip }}$ or equivalently $\Delta x^{\prime}$ is a signed quantity. This is not obvious from Eq. 3.4 which only calculates the absolute value of $\Delta x^{\prime}$. The sign of $\Delta x^{\prime}$ is determined by Eq. 3.5. The variable $\psi\left(s_{0}\right)$ corresponds to the phase advance of the triplet under study. If Eq. 3.5 gives a phase very close to the phase of the triplet then the sign of $\Delta x^{\prime}$ is positive. On the other hand, if Eq. 3.5 gives a phase very close to the phase of the triplet plus $\pi$ then the sign of the $\Delta x^{\prime}$ is negative.

\subsection{Analysis of First Turn Simulated Trajec- tories}

Before doing analysis in real orbits, simple simulation of orbits with skew quadrupole errors were made by modifying the software BMODEL that was 
mentioned earlier, and that will be described in Chapter 9.

The simulation is basically a trajectory obtained with Eq. 1.17, for example in the vertical plane. A similar orbit is created in the horizontal plane starting at the location of the skew quadrupole error, with an oscillation amplitude given by:

$$
\sqrt{2 J_{y}^{\text {Trip }}}=s k_{\text {err }} x^{e r r} \sqrt{\beta_{x}^{e r r}}
$$

where $s k_{e r r}$ is the simulated skew quadrupole error, $x^{e r r}$ is the horizontal position of the beam, $\beta_{x}^{e r r}$ is the horizontal beta function evaluated at the location of the skew quadrupole error. Notice that the amplitude of the induced oscillation in the vertical plane is proportional to the horizontal position of the beam at the place where the error is simulated.

The orbit is analyzed with the software MODULAR_IR2, as before, to find the action and phase before and after the skew quadrupole error. Since $J_{L}$ is zero in this case $J_{\text {Trip }}=J_{R}$ and $s k_{r e}$ can be easily calculated from Eq. 3.7, and their values verified as those values used to simulate the orbit.

Orbits were simulated with skew quadrupole errors at IR10, IR12 and IR2. MODULAR_IR2 was then used to extract the skew quadrupole errors from the simulated orbits. The extracted values were not exactly the same as the ones fed into the simulation, as can be seen in the third column Table 3.1. The reason lies in the fact that the program approximates $y^{s c}$ in Eq. 3.7 by the formula:

$$
y^{s c}=\sqrt{\frac{\beta_{y}^{s c}}{\beta_{y}^{b p m}}} y^{b p m}
$$


where $y^{b p m}$ is the beam position at the closest BPM to the skew quadrupole corrector, and $\beta_{y}^{b p m}$ is the beta function in the same BPM. The approximation here is to consider that the phase advance between the BPM and the skew quadrupole corrector is equal to zero.

\begin{tabular}{|c|c|c|c|c|}
\hline IR & $\begin{array}{c}\text { simulated } \\
\left(10^{-3} 1 / \mathrm{m}\right)\end{array}$ & $\begin{array}{c}\text { aprox. } \\
\left(10^{-3} 1 / \mathrm{m}\right)\end{array}$ & $\begin{array}{c}\text { linear inter. } \\
\left(10^{-3} 1 / \mathrm{m}\right)\end{array}$ & $\begin{array}{c}\text { fit all BPM's } \\
\left(10^{-3} 1 / \mathrm{m}\right)\end{array}$ \\
\hline IR10 & 1 & 0.973 & 0.989 & .99999 \\
IR12 & 3 & 3.09 & 3.79 & 2.9999 \\
IR2 & 6 & 5.99 & 7.55 & 6.0000 \\
\hline
\end{tabular}

Table 3.1: Three different approaches were used to find $y^{s c}$ in the program MODULAR_IR2

A second way of calculating $y^{s c}$ was by linear interpolation between the 2 closest BPM measurements. This approach corresponds to the fourth column in Table 3.1 where significant discrepancies with the simulated values can be seen. Linear interpolation doesn't seem a good method to work inside the IR. This might be due to the rapid change of the beta functions inside the IR. For example, in IR12 the vertical beta function of the BPMs used to do the linear interpolation is about $200 \mathrm{~m}$ while at the place where the error was simulated (between the BPMs) the vertical beta function is about $300 \mathrm{~m}$.

The third approach is to fit all BPMs measurements to the betatron equation in the closest arc to the skew quadrupole corrector under consideration. The model obtained in this way is then used to extrapolate $y^{s c}$. The values in the fifth column of Table 3.1 correspond to the skew errors calculated with this approach. The agreement between simulated values and extracted values 
in this case goes beyond the sixth significant figure. Later, we will see that there are almost no differences between the first and the third approach when they are applied to real data and hence they can be use indifferently.

\subsection{RHIC 2000 Difference First Turn Trajec- tory Analysis}

During the first RHIC runs in 1999 and 2000, difference trajectories produced by variations of dipole correctors strengths were taken on the first turn after injection, and stored for general optics studies. Those orbits can be found in the RHIC control system in the same root directory /operations/app_store/RhicOrbitDisplay/Blue/orbit_data/. For the analysis presented in this chapter the orbits were taken from the following subdirectories:

- /Run2001/June/Jun30EveDiffOrb/

- /Archive/April/Apr08Diff/

- /Archive/April/April29DiffOrbs/

- /Archive/May/May15Owl/

- /Archive/Run1999/

Several factors should be considered before using Eq. 3.7 to find $s k_{r e}$. First, not all orbits are adequate to find skew errors in all triplets. One of 
the reasons is the phase advance between the dipole corrector position and the triplet $\Delta \psi^{c t}$. Desirable phase advances are numbers close to odd multiples of $\pi / 2$, since then the orbit oscillation will be maximum at the triplet and the betatron oscillation induced in the opposite plane will also be maximum.

The second factor to consider is how close the dipole corrector used to produce the betatron oscillation is to the IR under study. If the dipole corrector is in the arc that immediately precedes the IR under study (left hand side for Blue ring and right hand side for Yellow ring) the strengths derived from the orbits will be called principal strengths, otherwise the strengths will be called secondary strengths.

The main difference between principal and secondary strengths lies in the induced orbit in the arc that immediately precedes the IR under study. For principal strengths, the arc induced orbit that immediately precedes the IR under study is zero, assuming no betatron oscillations are induced by other magnets in the arc. The same orbit segment is in general different from zero for the case of secondary strengths.

A third factor to take into account is the BPM measurement that is used in Eq. 3.7 to evaluate the strength needed in the skew quadrupole corrector. The BPM used for this purpose is usually the one just outside of the IR defined by the triplets which in Fig. 3.3 corresponds to either bo7-b3 or bi8-b3. This BPM has almost the same phase as its nearest skew quadrupole corrector, and therefore the procedure to estimate the position of the beam at the skew quadrupole corrector is simplified. When any of these BPMs is missing the 


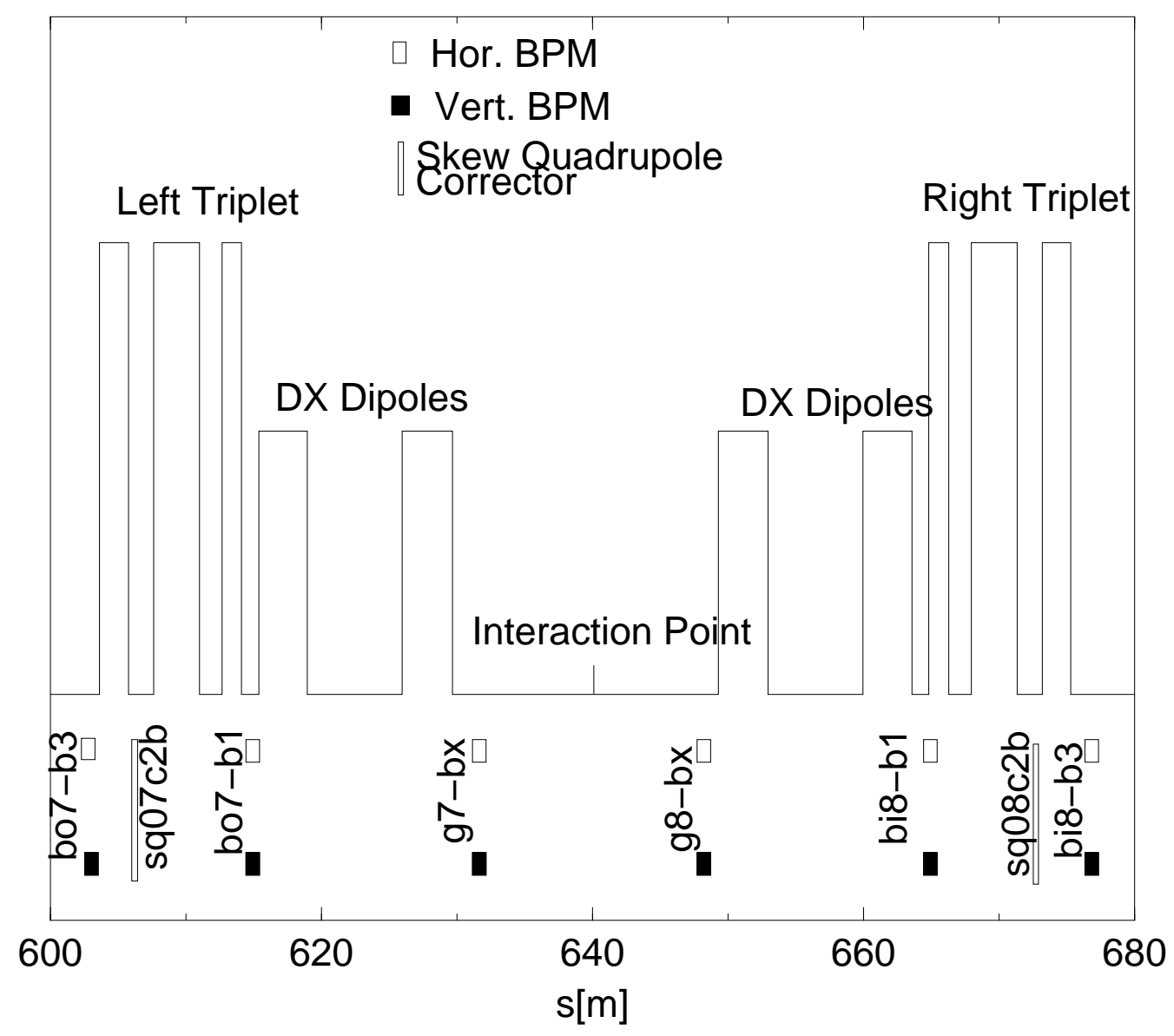

Figure 3.3: Schematic of 8 o'clock RHIC IR. The beam position at a particular skew quadrupole corrector is determined with the closest BPM to such corrector in each triplet. As an example, the closest BPM to the left skew quadrupole corrector $(\mathrm{sq07c} 2 \mathrm{~b})$ in the figure is bo7-b3. If this BPM doesn't give a proper reading of the beam position, bo7-b1 is used instead. 
corresponding b1 BPM can be used instead.

In principle, only one difference trajectory would be needed to extract the corrector strength required at a particular IR. However, several difference trajectories produced by different dipole correctors and different dipole corrector strengths are used to reduce the uncertainty in the measurement.

Principal and secondary strengths were used in IR8, IR10, IR12 and IR2 to do an average estimate of $s k_{r e}$ (see Table 3.2). The uncertainty corresponds to the variation of $s k_{r e}$ with the different orbits used to do the calculation. On the other hand $s k_{r e}$ strengths for IR4 and IR6 have big uncertainties when principal and secondary strengths are considered simultaneously $(0.45 \pm 0.27$ for IR4 and $.69 \pm .2$ for IR6). Table 3.2 shows the values of $s k_{r e}$ for IR4 and IR6 when only principal strengths are considered. The bigger uncertainties in

\begin{tabular}{|c|c|}
\hline IR & $s k_{r e}$ \\
& $\left(10^{-3} 1 / \mathrm{m}\right)$ \\
\hline IR8 & $0.67 \pm 0.07$ \\
IR10 & $1.00 \pm 0.15$ \\
IR12 & $0.18 \pm 0.03$ \\
IR2 & $0.99 \pm 0.14$ \\
IR4 & $0.63 \pm 0.06$ \\
IR6 & $0.60 \pm 0.15$ \\
\hline
\end{tabular}

Table 3.2: Measured Quadrupole Skew Errors(Run 2000) in Blue ring. More than 40 difference trajectories with kicks at different positions were used to obtain this table

$s k_{r e}$ when secondary strengths are considered might be due to the presence of gradient errors. Primary strengths are not sensitive to gradients errors since the position of the beam in the IR is zero or very close to zero, as discussed ear- 
lier. Nonlinear effects might also influence the differences observed in principal strength uncertainties and the secondary strengths uncertainties.

The orbit bump method (see Appendix D) was also used during the RHIC run 2000 to estimate $s k_{r e}$. The orbit bump method provided one strength for each triplet rather than the whole IR. these numbers, however, can be compared with the ones obtained on Table 3.2 by just adding the strengths found in the 2 triplets at each IR (see Section B.2). There is a reasonable good agreement between the values estimated with the orbit bump method and the method presented here as can be verified on Table D.1.

The values $y^{s c}$ or $x^{s c}$ at each IR needed to calculate the corresponding $s k_{r e}$ in Table 3.2 were found by fitting the BPM measurement in the the closest arc to the skew quadrupole corrector, as explained earlier in this chapter. Values of $s k_{r e}$ were also estimated using the approximate formula to find $y^{s c}$ from the closest BPM. The differences with Table 3.2 for most of the IRs is below $6 \%$. In other words, for real data, the fitting routine doesn't introduce a big improvement in the determination of the skew quadrupole errors. 


\section{Chapter 4}

\section{Correction of Linear Coupling during the RHIC 2001 Run}

This chapter has the purpose of illustrating the procedure followed during the RHIC 2001 run to perform local correction at the IRs making special emphasis on the importance of the orbit measurements during the correction procedure.

Global correction is also presented because the results obtained from this procedure favors the orbit measurements as a tool to perform local linear decoupling.

\subsection{Local Correction of Skew Quadrupole Er- rors at RHIC IRs}

As mentioned in Section 3.2 RHIC has corrector packages located at each triplet of the IRs. These corrector packages include skew quadrupole correctors that are used for local correction of linear coupling. 


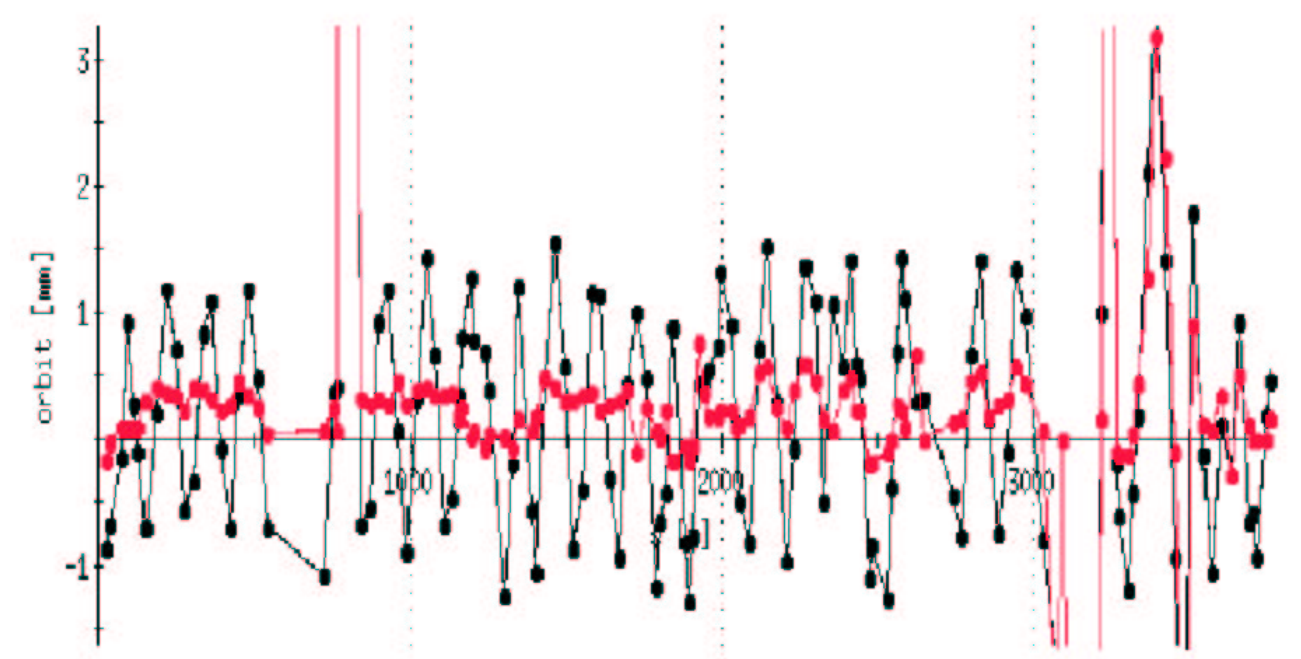

Figure 4.1: Induced orbits in the horizontal plane after creating a vertical orbit bump around the right triplet of 8 o'clock. Setting the skew quadrupole corrector to the calculated value the rms of the induced orbit is about 3 times smaller.

The values at which such correctors should be set has been determined from orbit measurements in the previous chapter (see Table 3.2). Such values can be put in either the left triplet skew quadrupole corrector or the right triplet skew quadrupole corrector to locally correct the coupling effect at a specific IR. It is also possible to set the correctors such that their sum equals the skew quadrupole error (see demonstration in Appendix B.2) . This would guarantee that the correction is effective at least outside the IRs. However, other methods like the orbit bump method (see Appendix D) and the method that will be presented in the next chapter make predictions for each corrector individually. The actual skew quadrupole corrector setting was done during the RHIC 2001 run (Gold Run). The procedure was basically to choose some 
starting value for the corrector of a particular triplet. Then, orbit bumps were done around such triplet while monitoring the induced orbit in the opposite plane (see Fig. 4.1). Several settings were tried and the one that give the minimum induced orbit in the opposite plane was chosen. This procedure was followed for IR8, IR10 and IR2. The resultant settings were consistent with the results of the orbit bump method and first turn analysis as well (see Table 4.1). The other correctors were then set in 1 step by using the predicted values by the two methods.

\begin{tabular}{|c|c|c|}
\hline Triplet & $\begin{array}{c}s k_{r e} \\
\text { Actual Setting } \\
10^{-3} 1 / \mathrm{m}\end{array}$ & $\begin{array}{c}s k_{r e} \text { all IR } \\
\text { Mesured Values } \\
10^{-3} 1 / \mathrm{m}\end{array}$ \\
\cline { 1 - 2 } 1 & -0.20 & \multirow{2}{*}{0.99} \\
\hline 2 & 1.20 & \multirow{2}{*}{0.63} \\
\hline 3 & 0.320 & \multirow{2}{*}{0.60} \\
\hline 4 & 0.320 & \multirow{2}{*}{0.67} \\
\hline 5 & 0.40 & \multirow{2}{*}{1.00} \\
\hline 6 & 0.10 & \multirow{2}{*}{0.18} \\
\hline 7 & -0.80 & \\
\hline 8 & 1.30 & \multicolumn{2}{|c|}{} \\
\hline 10 & 0.65 & \\
\hline 11 & 0.09 & \\
\hline 12 & 0.09 & \\
\hline
\end{tabular}

Table 4.1: Skew quadrupole correctors at IR8, IR10 and IR2 were set by using the orbit bump method on line. Since the settings obtained in this way were consistent with the measured values, the remaining correctors were set in one step according the measured values. In order to compare the second and the third column of this table it is neccesary to add the strengths of the left and right triplets of each IR. 


\subsection{Global Correction of Linear Coupling}

We have seen that the orbit bumps (Fig. 4.1) made during the local correction procedure indicates that the measurements from first turn orbits are a reliable way of finding the strengths of the skew quadrupole correctors.

Comparisons with magnetic measurements is another strong indication that the orbit measurements indeed give accurate numbers to set the local skew quadrupole correctors and it will be described on detail on Chapter 6 .

Another possible indication that favors orbit measurements is the global decoupling measurements made during the RHIC 2001 run, some of which will be presented in the following paragraphs.

RHIC has 3 families of skew quadrupole correctors in the arcs located at an appropriate phase advance between them to facilitate the global correction of linear coupling. The procedure to do the correction is to measure the coupled tunes but instead of varying the tune knobs as was shown in Section 1.8, the skew quadrupole family strengths are varied one by one. Fig. 4.2 shows a typical tune scan with skew quadrupole family \#1 while the other two families are set to fixed values. The point at which the tunes are the closer determines the optimal strength setting for the skew quadrupole family under study and also the $\Delta q_{\text {min }}$. In principle $\Delta q_{\text {min }}$ should be zero if the quadrupole families are properly adjusted. Experimental results show that this is not always the

case and that there is always a $\Delta q_{\min }$ different from zero (This is evident in Fig. 4.2) that changes with the settings of the local skew quadrupole correctors. 


\section{Decoupling Scan - Family \#1}

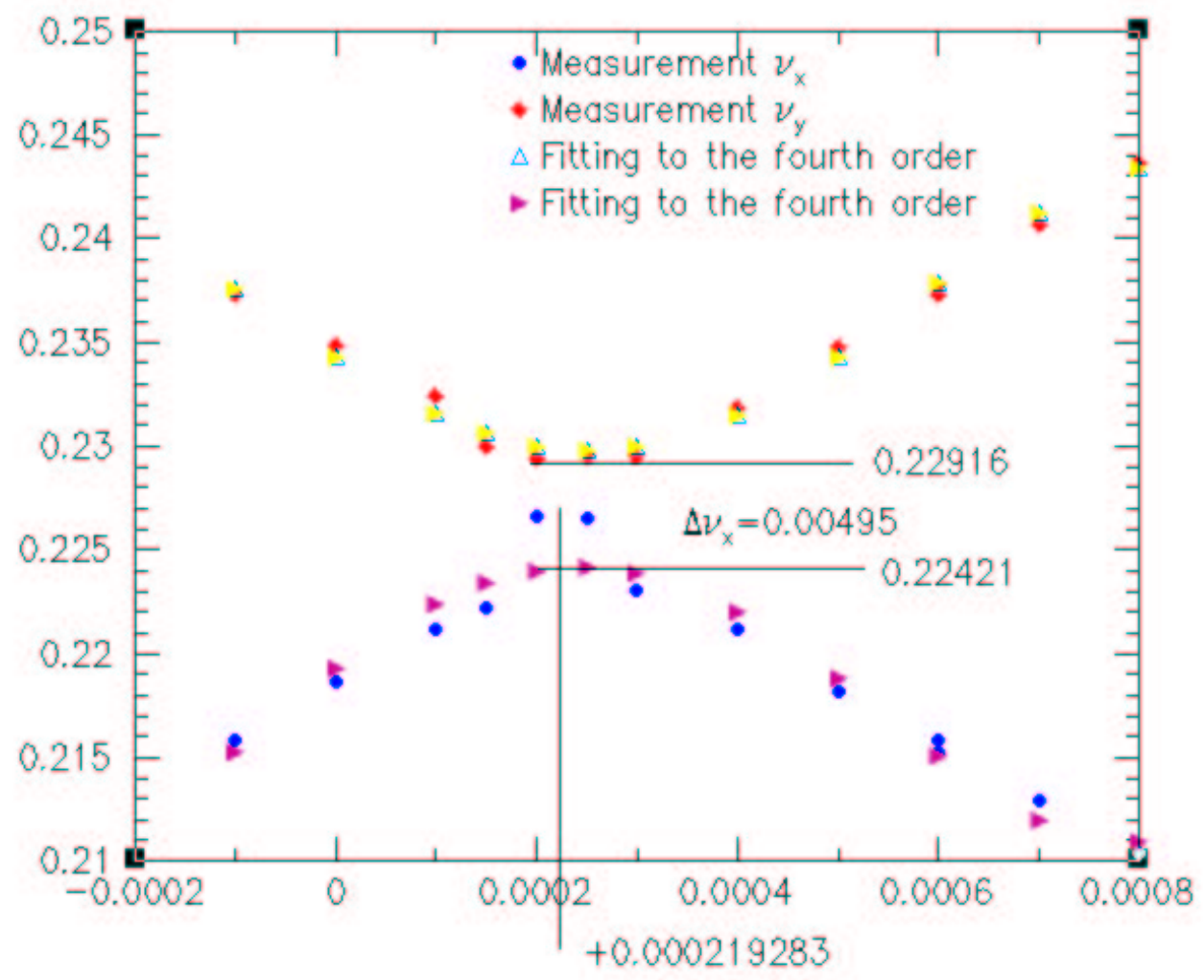

Figure 4.2: Horizontal and Vertical tunes while the skew quadrupole strength of family \#1 is varied ( Jun 4 RHIC 2001 run ).

As example I will cite the measurements of $\Delta q_{\min }$ done on May 31 and July 12 in the RHIC 2001 run.

The measured $\Delta q_{\min }$ on May 31 of 2001 was about 0.007 without any local correction. The value of 0.007 is the minimum $\Delta q_{\min }$ obtained for all the measurements performed before the local linear coupling correction.

After the local linear coupling correction was done in all Blue ring IRs, 
$\Delta q_{\min }$ was measured and its value was 0.0005 , one order of magnitude smaller than the minimum value obtained before the local linear coupling correction.

The strengths used in the skew quadrupole families to reach $\Delta q_{\min }$ also indicate that the sources of linear coupling were reduced after the local linear coupling correction was done. The skew quadrupole families were set to Family $\# 1=0$, Family $\# 2=0.0012^{1}$, Family \#3 = 0 on May 31 of 2001 (before decoupling) and the same families were set to Family \#1 = 0, Family \#2 = -0.0006 , Family $\# 3=0$ (after decoupling).

\footnotetext{
${ }^{1}$ One of the power supplies for this family of skew quadrupoles was broken when this family was set. This means that only 12 out 16 quadrupole were on, which in turns means that the effective strength was 0.0009
} 


\section{Chapter 5}

\section{Linear Errors from Closed Orbit Analysis}

First turn orbits are only available at injection and they are very susceptible to random fluctuations. Closed orbits, on the other hand, are available at injection, storage and through the ramp and since closed orbits are obtained from the average of many turns orbits the chances of having random fluctuations are minimized.

Closed orbits are also possible particle trajectories and hence the analysis done with first turn orbits in Chapter 3 should also be valid for closed orbits. This is partially true because the cases that were studied in Chapter 3 assume the presence of only skew quadrupole errors. A more complete description should also include gradient errors and non linear errors.

In this chapter, a general technique that will allow to measure linear and non linear errors at RHIC IRs from orbit measurement will be developed. Even though, the technique is applied only to closed orbits in this chapter, the same 

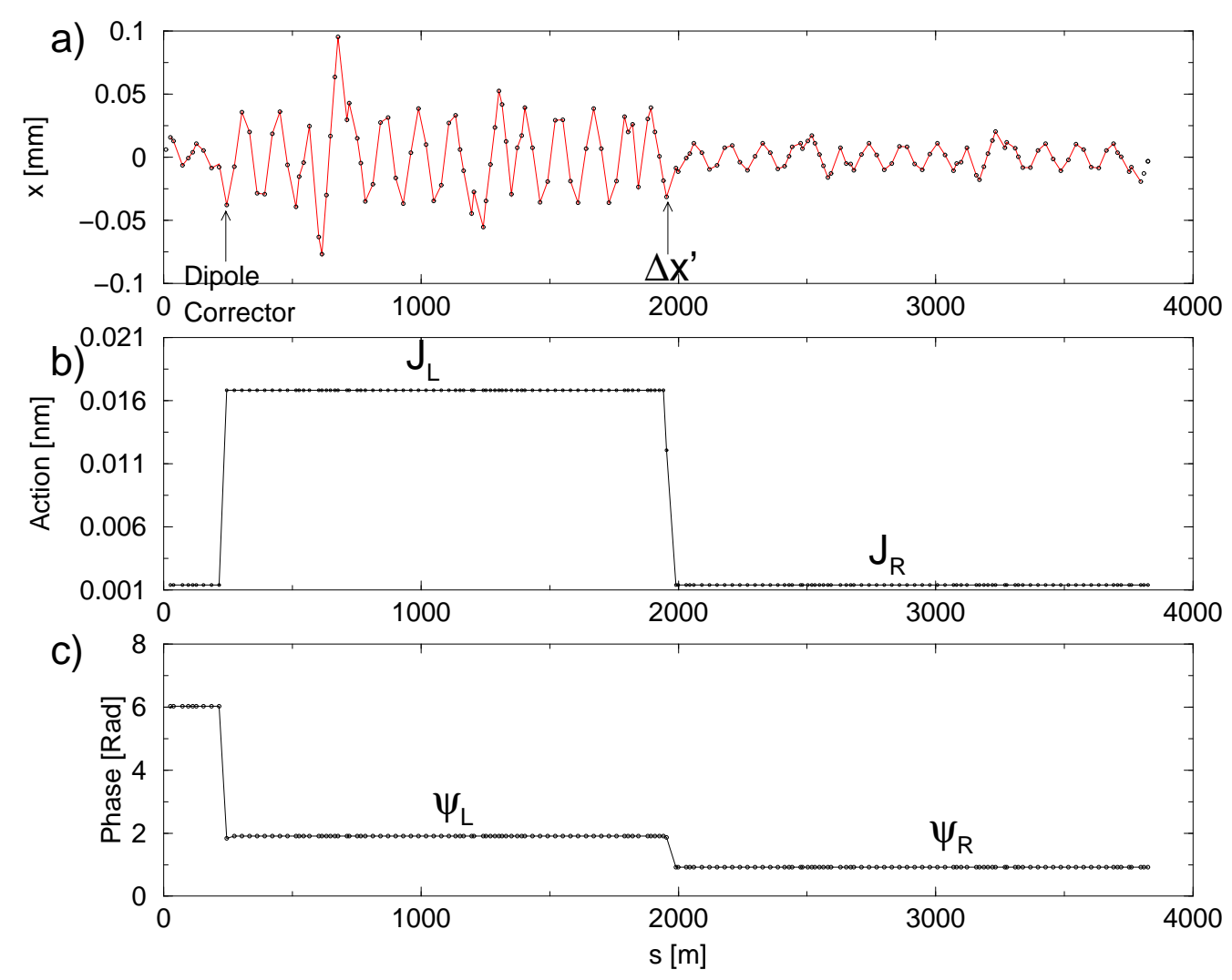

Figure 5.1: a) Simulated RHIC orbit excited with a dipole corrector at $s=$ $215 \mathrm{~m}$ and with a magnetic kick error $\Delta x^{\prime}$ at $\mathrm{s}=1945 \mathrm{~m}$. b) Action analysis of the orbit in a). c) Phase analysis of the orbit in a).

technique can be used indifferently for first turn orbits and turn by turn orbits.

\subsection{Closed Orbits and Action and Phase Anal- ysis}

It was mentioned in the introduction of this Chapter that closed orbits are also possible particle trajectories and hence analysis done for particle trajec- 
tories are also valid for closed orbits. In this section, it will be shown that the previous statement is valid at least for Eq. 3.4, which was originally deduced for orbit trajectories.

Start by assuming that there is a magnetic error $\Delta x^{\prime}$ located at phase $\psi_{I}$, and that a dipole corrector has been activated far away from the magnetic error (see Fig. 5.1). Using superposition principle and the fact that between the errors it is possible to use the designed lattice functions (see Section 1.5) the resultant closed orbit for $\psi(s)<\psi_{I}$ is:

$$
\begin{aligned}
\sqrt{2 J_{L} \beta(s)} \sin \left(\psi(s)-\psi_{L}\right) & =A \sqrt{\beta(s)} \sin \left(\psi(s)-\psi_{A}\right) \\
& +\frac{\Delta x^{\prime} \sqrt{\beta_{I} \beta(s)}}{2 \sin (\pi Q)} \cos \left(\psi_{I}-\psi(s)-\pi Q\right)
\end{aligned}
$$

where $J_{L}$ and $\psi_{L}$ are the action and phase associated with the resultant closed orbit for $\psi(s)<\psi_{I}$ (see Fig. 5.1), A and $\psi_{A}$ are the amplitude and phase associated with the closed orbit due to the dipole corrector, $\beta_{I}$ is the beta function at the place where the magnetic error is, and $Q$ corresponds to the tune of the machine.

It is convenient to write the contribution of the magnetic error to the closed orbit, $x_{D K}(s)$, in its sinusoidal form. That is:

$$
\begin{aligned}
x_{D K}(s) & =\frac{\Delta x^{\prime} \sqrt{\beta_{I} \beta(s)}}{2 \sin (\pi Q)} \cos \left(\psi_{I}-\psi(s)-\pi Q\right) \\
& =\frac{\Delta x^{\prime} \sqrt{\beta_{I} \beta(s)}}{2 \sin (\pi Q)} \sin \left(\psi_{I}-\psi(s)-\pi Q+\pi / 2\right)
\end{aligned}
$$




$$
\begin{aligned}
& =-\frac{\Delta x^{\prime} \sqrt{\beta_{I} \beta(s)}}{2 \sin (\pi Q)} \sin \left(\psi(s)-\chi_{L}\right) \\
& =-\sqrt{2 J_{I} \beta(s)} \sin \left(\psi(s)-\chi_{L}\right)
\end{aligned}
$$

where, in order to simplify the notation, $\chi_{L}$ and $J_{I}$ has been defined in the following way:

$$
\chi_{L}=\pi / 2-\pi Q+\psi_{I}
$$

and

$$
J_{I}=\frac{\Delta x^{2} \beta_{I}}{8 \sin ^{2}(\pi Q)}
$$

Replacing Eq. 5.2 and the definitions of $\chi_{L}$ and $J_{I}$ in Eq. 5.1, we have:

$$
\begin{aligned}
\sqrt{\left(2 J_{L} \beta(s)\right) \sin \left(\psi(s)-\psi_{L}\right)=} & A \sqrt{(\beta(s))} \sin \left(\psi(s)-\psi_{A}\right) \\
- & \sqrt{2 J_{I} \beta(s)} \sin \left(\psi(s)-\chi_{L}\right) \\
= & \sqrt{\beta(s)}\left[A\left(\sin \psi(s) \cos \psi_{A}-\sin \psi_{A} \cos \psi(s)\right)\right. \\
& \left.-\sqrt{2 J_{I}}\left(\sin \psi(s) \cos \chi_{L}-\sin \chi_{L} \cos \psi(s)\right)\right] \\
= & \sqrt{\beta(s)}\left[\left(A \cos \psi_{A}-\sqrt{2 J_{I}} \cos \chi_{L}\right) \sin \psi(s)\right. \\
& \left.\left(-A \sin \psi_{A}+\sqrt{2 J_{I}} \sin \chi_{L}\right) \cos \psi(s)\right]
\end{aligned}
$$

The last equation is compared with:

$$
\sqrt{2 J_{L} \beta(s)} \sin \left(\psi(s)-\psi_{L}\right)=\sqrt{2 J_{L} \beta(s)}\left[\sin \psi(s) \cos \psi_{L}-\sin \psi_{L} \cos \psi(s)\right]
$$

to obtain: 


$$
\begin{gathered}
\sqrt{2 J_{L}} \cos \psi_{L}=A \cos \psi_{A}-\sqrt{2 J_{I}} \cos \chi_{L} \\
-\sqrt{2 J_{L}} \sin \psi_{L}=-A \sin \psi_{A}+\sqrt{2 J_{I}} \sin \chi_{L}
\end{gathered}
$$

Similar relations can be obtained for $\psi(s)>\psi_{I}$. As before, the superposition principle is used to find the resultant closed orbit.

$$
\begin{aligned}
\sqrt{2 J_{R} \beta(s)} \sin \left(\psi(s)-\psi_{R}\right) & =A \sqrt{\beta(s)} \sin \left(\psi(s)-\psi_{A}\right) \\
& +\frac{\Delta x^{\prime} \sqrt{\beta_{I} \beta(s)}}{2 \sin (\pi Q)} \cos \left(\psi(s)-\psi_{I}-\pi Q\right)
\end{aligned}
$$

where $J_{R}$ and $\psi_{R}$ are the action and phase associated with the resultant closed orbit for $\psi(s)>\psi_{I}$ and $\mathrm{A}$ and $\psi_{A}$ are the amplitude and phase associated with the closed orbit due to the dipole corrector.

It is convenient to write the contribution of the magnetic error to the closed orbit, $x_{D K}(s)$, in its sinusoidal form. That is:

$$
\begin{aligned}
x_{D K}(s) & =\frac{\Delta x^{\prime} \sqrt{\beta_{I} \beta(s)}}{2 \sin (\pi Q)} \cos \left(\psi(s)-\psi_{I}-\pi Q\right) \\
& =\frac{\Delta x^{\prime} \sqrt{\beta_{I} \beta(s)}}{2 \sin (\pi Q)} \sin \left(\psi(s)-\psi_{I}-\pi Q+\pi / 2\right) \\
& =\frac{\Delta x^{\prime} \sqrt{\beta_{I} \beta(s)}}{2 \sin (\pi Q)} \sin \left(\psi(s)-\chi_{R}\right) \\
& =\sqrt{2 J_{I} \beta(s)} \sin \left(\psi(s)-\chi_{R}\right)
\end{aligned}
$$


where $\chi_{R}=\pi Q+\psi_{I}-\pi / 2$. Replacing Eq. 5.9 in Eq. 5.8 we have:

$$
\begin{aligned}
\sqrt{\left(2 J_{R} \beta(s)\right) \sin \left(\psi(s)-\psi_{R}\right)=} & A \sqrt{(\beta(s)) \sin \left(\psi(s)-\psi_{A}\right)}+ \\
+ & \sqrt{2 J_{I} \beta(s)} \sin \left(\psi(s)-\chi_{R}\right) \\
= & \sqrt{\beta(s)}\left[A\left(\sin \psi(s) \cos \psi_{A}-\sin \psi_{A} \cos \psi(s)\right)\right. \\
& \left.+\sqrt{2 J_{I}}\left(\sin \psi(s) \cos \chi_{R}-\sin \chi_{R} \cos \psi(s)\right)\right] \\
= & \sqrt{\beta(s)}\left[\left(A \cos \psi_{A}+\sqrt{2 J_{I}} \cos \chi_{R}\right) \sin \psi(s)\right. \\
& \left.\left(-A \sin \psi_{A}-\sqrt{2 J_{I}} \sin \chi_{R}\right) \cos \psi(s)\right]
\end{aligned}
$$

The last equation is compared with:

$$
\sqrt{2 J_{R} \beta(s)} \sin \left(\psi(s)-\psi_{R}\right)=\sqrt{2 J_{R} \beta(s)}\left[\sin \psi(s) \cos \psi_{R}-\sin \psi_{R} \cos \psi(s)\right]
$$

to obtain:

$$
\begin{gathered}
\sqrt{2 J_{R}} \cos \psi_{R}=A \cos \psi_{A}+\sqrt{2 J_{I} R} \cos \chi_{R} \\
-\sqrt{2 J_{R}} \sin \psi_{R}=-A \sin \psi_{A}-\sqrt{2 J_{I} R} \sin \chi_{R}
\end{gathered}
$$

Combining Eq. 5.7 and Eq. 5.12:

$$
\begin{aligned}
\sqrt{2 J_{L}} \cos \psi_{L}-\sqrt{2 J_{R}} \cos \psi_{R} & =-\sqrt{2 J_{I}} \cos \chi_{L}-\sqrt{2 J_{I}} \cos \chi_{R} \\
& =-\sqrt{2 J_{I}}\left(\cos \left(\chi_{L}\right)-\cos \left(\chi_{R}\right)\right)
\end{aligned}
$$




$$
\begin{aligned}
-\sqrt{2 J_{L}} \sin \psi_{L}+\sqrt{2 J_{R}} \sin \psi_{R} & =\sqrt{2 J_{I}} \sin \chi_{L}+\sqrt{2 J_{I}} \sin \chi_{R} \\
& =\sqrt{2 J_{I}}\left(\sin \chi_{L}+\sin \chi_{R}\right)
\end{aligned}
$$

Squaring and adding the 2 previous results lead to:

$$
\begin{aligned}
2 J_{L}+2 J_{R}-2\left(2 \sqrt{J_{L} J_{R}}\right)\left(\cos \psi_{L} \cos \psi_{R}+\sin \psi_{L} \sin \psi_{R}\right) & =4 J_{I} \\
& +4 J_{I} \cos \chi_{L} \cos \chi_{R} \\
& \\
& +4 J_{I} \sin \chi_{L} \sin \chi_{R} \\
J_{L}+J_{R}-2 \sqrt{J_{L} J_{R}} \cos \left(\psi_{L}-\psi_{R}\right) & =2 J_{I} \\
& +2 J_{I} \cos \left(\chi_{L}-\chi_{R}\right)
\end{aligned}
$$

where $\chi_{L}-\chi_{R}=\pi-2 \pi Q$ according to the previous definitions. Inserting this result in Eq. 5.14 we have:

$$
\begin{aligned}
J_{L}+J_{R}-2 \sqrt{J_{L} J_{R}} \cos \left(\psi_{L}-\psi_{R}\right) & =J_{I}(2-2 \cos (2 \pi Q)) \\
& =2 J_{I}\left(1-\cos ^{2} \pi Q-\sin ^{2} \pi Q\right) \\
& =2 J_{I}\left(1-\left(1-2 \sin ^{2} \pi Q\right)\right) \\
& =4 J_{I} \sin ^{2}(\pi Q)
\end{aligned}
$$

From the last equation and the definition of $J_{I}$ in Eq. 5.4 it is finally possible to relate $\Delta x^{\prime}$ with the measured action and phases by the equation: 


$$
\Delta_{x}^{\prime}=\sqrt{\frac{2 J_{L}+2 J_{R}-4 \sqrt{J_{L} J_{R}} \cos \left(\psi_{L}-\psi_{R}\right)}{\beta_{I}}}
$$

which is the same result obtained for first turn orbits (Eq. 3.4) as expected.

The fact that the same equation to obtain $\Delta x^{\prime}$ is valid for first turn orbits and for closed orbits allows the same software to be used for both cases. The only difference arises when errors are calculated at IR6. The reason is the following: to calculate errors in IR6, orbit information in the arc that goes from IR4 and IR6 and orbit information between the arc that goes from IR6 to IR8 are required. In first turn orbits, measurements in the arc between IR6 and IR8 of the second turn are required to extract errors at IR6. Closed orbits, on the other hand, use the arc between IR6 and IR 8 contained in the only turn orbit to estimate errors at IR6.

\subsection{Determination of Linear Errors from In- dividual Closed Orbits}

During the RHIC 2000 run, a few difference closed orbits were taken for diverse purposes. To find the corrector strengths from these orbits, Eq. 3.4 and Eq. 3.7 were used in the same way they were used for first turn trajectories in Chapter 3. The results can be seen in Table 5.1. Measurement at IR2 and IR6 were not possible due to multiple reasons like BPMs not working properly at the IRs or poor fits. In general there are a well identified set of reasons to discard an IR measurement and they are systematically check not only here 


\begin{tabular}{|r|c|}
\hline IR & $s k_{r e}$ \\
& $\left(10^{-3} 1 / \mathrm{m}\right)$ \\
\hline 8 & $0.54 \pm 0.18$ \\
10 & $0.56 \pm 0.41$ \\
12 & $0.18 \pm 0.07$ \\
4 & $0.35 \pm 0.22$ \\
\hline
\end{tabular}

Table 5.1: skew errors from closed orbit analysis (RHIC 2000 orbits). No gradient errors are considered for this analysis.

but in all the analysis that involve determination of errors with the aid of the action and phase analysis. Appendix E summarizes the common failures found in the difference orbits that make invalid an IR measurement.

One important observation about the results of Table 5.1 is the large uncertainties associated with the skew quadrupole errors. In Section 3.4, I made the distinction between principal and secondary strengths based on the value of the action previous to the error. If such action was zero the strength would be a principal strength, otherwise it would be a secondary strength. Since it is unlikely to have arcs with action equal or closed to zero in closed orbits, most of the strengths that can be extracted from closed orbits are secondary strengths.

Strengths extracted from first turn orbits also had bigger uncertainties when the secondary strengths were taken into account (see Section 3.4). This effect might be due to the presence of gradient errors simultaneously with the presence of skew quadrupole errors at the IRs. Eq. 3.7 to find $s k_{r e}$ was deduced under the assumption that no gradient errors were present, this works well for principal strengths, because the orbit before the error is zero or close to zero, 
and the contribution of the gradient term is zero even if the gradient itself is different from zero.

For secondary strengths the contribution of the gradient error to the total error $\Delta x^{\prime}$ can be significant and must be included in the calculations. Eq. 1.56 allows to find the skew error $A_{1}$ and the gradient error , $B_{1}$, as function of the measured quantities $\Delta x^{\prime}, \Delta y^{\prime}$, the horizontal position of the beam , $x$, and the vertical position of the beam , $y$, at the position where $\Delta x^{\prime}$ and $\Delta y^{\prime}$ are evaluated. Such relations are:

$$
\begin{aligned}
& A_{1}=\frac{y \Delta x^{\prime}+x \Delta y^{\prime}}{x^{2}+y^{2}} \\
& B_{1}=\frac{y \Delta y^{\prime}-x \Delta x^{\prime}}{x^{2}+y^{2}},
\end{aligned}
$$

Using the same orbits that were used to produce Table 5.1, Eq. 5.17 leads to Table 5.2.

\begin{tabular}{|c|c|c|}
\hline IR & $\begin{array}{c}A_{1} \\
\left(10^{-3} 1 / \mathrm{m}\right)\end{array}$ & $\begin{array}{c}B_{1} \\
\left(10^{-3} 1 / \mathrm{m}\right)\end{array}$ \\
\hline 8 & $0.62 \pm 0.14$ & $0.22 \pm 0.61$ \\
10 & $1.08 \pm 0.35$ & $1.38 \pm 1.16$ \\
12 & $0.19 \pm 0.07$ & $0.09 \pm 0.74$ \\
4 & $0.65 \pm 0.53$ & $1.76 \pm 2.80$ \\
\hline
\end{tabular}

Table 5.2: Skew and Gradients obtained from Closed Orbit Analysis. Orbits taken during the 2001 RHIC run.

Except for IR4, Table 5.2 shows a reduction in the uncertainty associated with the skew quadrupole error $A_{1}$ in the IRs when compared with Table 5.1. 
Also, the average values are much closer to the ones found from first turn orbits (Table 3.2). However $B_{1}$, the gradient error, has very big uncertainties associated with it.

Later, we will see that the big uncertainties associated with the gradient errors have their origin in the fact that $B_{1}$ is not the same in both planes; there will be a $B_{1}^{x}$ and $B_{1}^{y}$.

$A_{1}$ happens to be equal to $s k_{r e}$, the ramp editor value at which the skew quadrupole corrector must be set to cancel the coupling. There is a double change of sign here. The corrector needed by definition should cancel the effect of the original $A_{1}$ error. This is the first change of sign. The second change of sign is obtained due to the way $s k_{r e}$ has been defined (see Appendix F).

\subsection{Simulation of Gradient and Skew Errors}

Closed orbit simulations were performed with a simple program based on the closed orbit equations. In this program there are basically two sources for closed orbit displacements. The first source is the dipole corrector that in the real experiment is set to a relatively large value and that will mainly determine the closed orbit. The second source is the error that is to be simulated and that will produce another closed orbit that can be superimposed on the one produced by the dipole corrector. This approach is exact: no approximation has been made at this point. The procedure is correct even for kicks from non-dipole sources, according to the discussions presented in Section 1.5. 
Magnetic errors other than dipole kicks depends on the beam position , either horizontal or vertical at the position where the errors are. For closed orbits, the beam positions at the location of the errors also depends on the errors themselves. The simple program used to produce closed orbits need as an input these beam positions before the program can produce the closed orbit. The calculations of the beam position at the places where the errors are were done only for linear errors, and they will be reproduced partially in the following paragraphs.

In general, the orbits produced by the dipole kick and the linear error together can be written as:

$$
\begin{aligned}
x(s) & =\frac{\Delta x^{\prime} \sqrt{\beta_{x}(s) \beta_{x}\left(s_{0}\right)} \cos \left[\left|\psi(s)-\psi\left(s_{0}\right)\right|-\pi Q\right]}{2 \sin \pi Q} \\
& +\frac{\left(A_{1} y\left(s_{0}\right)-B_{1} x\left(s_{0}\right)\right) \sqrt{\beta_{x}(s) \beta_{x}\left(s_{0}\right)} \cos \left[\left|\psi(s)-\psi\left(s_{0}\right)\right|-\pi Q\right]}{2 \sin \pi Q}
\end{aligned}
$$

and

$$
\begin{aligned}
y(s) & =\frac{\Delta y^{\prime} \sqrt{\beta_{y}(s) \beta_{y}\left(s_{0}\right)} \cos \left[\left|\psi(s)-\psi\left(s_{0}\right)\right|-\pi Q\right]}{2 \sin \pi Q} \\
& +\frac{\left(A_{1} x\left(s_{0}\right)+B_{1} y\left(s_{0}\right)\right) \sqrt{\beta_{x}(s) \beta_{x}\left(s_{0}\right)} \cos \left[\left|\psi(s)-\psi\left(s_{0}\right)\right|-\pi Q\right]}{2 \sin \pi Q}
\end{aligned}
$$

where $\Delta x^{\prime}$ and $\Delta y^{\prime}$ are the dipole kicks provided by the correctors and $A_{1}$ and $B_{1}$ the linear coefficients of the errors. Evaluating Eq. 5.18 and Eq. 5.19 at $s_{0}$ 
we have:

$$
\begin{aligned}
x\left(s_{0}\right)= & \frac{\Delta x^{\prime} \beta_{x}\left(s_{0}\right) \cos [\pi Q]}{2 \sin \pi Q} \\
& +\frac{\left(A_{1} y\left(s_{0}\right)-B_{1} x\left(s_{0}\right)\right) \beta_{x}\left(s_{0}\right) \cos [\pi Q]}{2 \sin \pi Q} \\
y\left(s_{0}\right)= & \frac{\Delta y^{\prime} \beta_{y}\left(s_{0}\right) \cos [\pi Q]}{2 \sin \pi Q} \\
& +\frac{\left(A_{1} x\left(s_{0}\right)+B_{1} y\left(s_{0}\right)\right) \beta_{x}\left(s_{0}\right) \cos [\pi Q]}{2 \sin \pi Q}
\end{aligned}
$$

This is a $2 \times 2$ linear equation system in the variables $x\left(s_{0}\right)$ and $y\left(s_{0}\right)$, which can be solved with standard methods. Once $x\left(s_{0}\right)$ and $y\left(s_{0}\right)$ are known Eq. 5.18 and Eq. 5.19 can be evaluated at each point in the ring.

The simple simulator based on Eq. 5.18 and Eq. 5.19 was used routinely to test the software to find strengths with closed orbits.

\subsection{Dipole Correctors and IR errors}

The simulator was also used to study how the difference phase advance between the dipole corrector used to excite the betatron oscillation and the IR under study, $\Delta \psi^{c i}$, would affect the determination of skew quadrupole errors.

With this objective in mind, orbits with a skew error and a gradient error located at the skew quadrupole corrector of triplet 9 were simulated using many different dipole correctors to excite the betatron oscillation. The simulated orbits were then analyzed with the software MODULAR_IR2 which extracts the skew errors and gradient errors. Results can be seen in Table 5.3. 


\begin{tabular}{|l|c|c|c|}
\hline Corrector & diff_phase & $\begin{array}{c}A_{1} \\
\left(10^{-3} 1 / \mathrm{m}\right)\end{array}$ & $\begin{array}{c}B_{1} \\
\left(10^{-3} 1 / \mathrm{m}\right)\end{array}$ \\
\hline bi4-th9 & 0.01 & 1.000 & -0.984 \\
bi12-th17 & 0.03 & 0.985 & -0.974 \\
bi4-th13 & 0.11 & 0.985 & -0.993 \\
bi4-th5 & 0.17 & 0.989 & -1.025 \\
bo3-th12 & 0.29 & 0.978 & -1.022 \\
bi5-th9 & 0.35 & 0.970 & -1.024 \\
bo2-th18 & 0.39 & 0.992 & -0.961 \\
bi5-th13 & 0.45 & 0.102 & -0.964 \\
\hline
\end{tabular}

Table 5.3: Effect of the difference phase advance between the dipole corrector used to excite the betatron oscillation and the IR under study in the determination of linear errors. diff_phase is not exactly the phase advance between corrector and and IR but rather it is a normalized number that indicate how close to the IR the closed orbit has a maximum (see Chapter 9 ). All the orbits were simulated with $A_{1}=0.0011 / \mathrm{m}$ and $B_{1}=-0.0011 / \mathrm{m}$

In theory, the values in the table should be exactly equal to the values used in the simulation. The small differences are due to the fact that MODULAR_IR2 approximates the beam position at the skew quadrupole corrector with Eq. 3.9, as mentioned previously. Some dependence between $\Delta \psi^{c i}$ and the determination of the linear errors by MODULAR_IR2 was expected due to this approximation, but the variations are minimal. This is due in part to the fact that the simulations were done introducing errors exactly at the left skew quadrupole corrector of the IR, which is also the place where MODULAR_IR2 calculates the equivalent skew quadrupole error.

The real situation is that errors will be distributed in all 6 quadrupoles of the IR, and hence the determination of the equivalent skew quadrupole 
error is expected to be sensitive to $\Delta \psi^{c i}$. Table 5.4 show results of simulations where the skew error was located in the right triplet, while MODULAR_IR2 extracted the equivalent skew quadrupole error at the left skew quadrupole corrector.

\begin{tabular}{|l|c|c|c|}
\hline Corrector & diff_phase & $\begin{array}{c}A_{1} \\
\left(10^{-3} 1 / \mathrm{m}\right)\end{array}$ & $\begin{array}{c}B_{1} \\
\left(10^{-3} 1 / \mathrm{m}\right)\end{array}$ \\
\hline bi4-th9 & 0.01 & 0.704 & -1.021 \\
bi12-th17 & 0.03 & 1.214 & -0.909 \\
bi4-th13 & 0.11 & 1.238 & -1.025 \\
bi4-th5 & 0.17 & 1.167 & -0.634 \\
bo3-th12 & 0.29 & 1.282 & -0.602 \\
bi5-th9 & 0.35 & 1.357 & -0.592 \\
bo2-th18 & 0.39 & 1.058 & -1.688 \\
bi5-th13 & 0.45 & 0.417 & -1.654 \\
\hline
\end{tabular}

Table 5.4: Closed orbits with skew errors in the right triplet of IR10 were simulated and MODULAR_IR2 was then used to extract the errors from the simulated orbits. As before the linear errors used in the simulation were $A_{1}=$ $0.0011 / \mathrm{m}$ and $B_{1}=-0.0011 / \mathrm{m}$.

In this case the difference between the simulated values and those extracted with MODULAR_IR2 are significant, and they increase as the phase advance gets closer to 0.5. According to Table 5.4 it seems that a phase advance of less than 0.1 will be adequate to determine linear errors. A program called BPHASE_ADV for the Blue ring, and YPHASE_ADV for the Yellow ring were written to calculate diff_phase between a particular IR and all dipole correctors in the ring. From the output of these programs it is then easy to choose the dipole correctors with the proper phase advance to a specific IR. 


\subsection{Effect of Nonlinear Errors in the Determi- nation of Linear Errors}

In Section 5.2 we have seen that gradient errors play an important role in the determination of the skew quadrupole errors. It is then logical to ask if nonlinear errors are also important in the determination of either skew quadrupole errors and gradient errors as well. In the following sections, some of the experiments that were done with this purpose in mind are presented.

A very important aspect of this section is that will be the foundation for the development of a novel technique to find nonlinear errors at the IRs from beam measurements that will be completely describe in Chapter 7 . This technique will also facilitate the determination and automation of linear errors at the IRs.

\subsubsection{Experiment}

The experiment is basically to shift the closed orbit by changing the strength of a specific dipole corrector. This procedure was followed for protons in the RHIC 2001 run where the strength was changed four times. Similar experiments were made with gold ions in the RHIC 2001 run, but the strength of the dipole correctors used was changed only twice.

As before, difference orbits are created respect to a baseline before doing the action and phase analysis as explained in Section 2.2. 


\subsubsection{Analysis}

Since for this case the orbits to analyze are closed orbits, the resolution of the measurements is better than for first turn trajectories. In particular, the 2 BPMs located in the center of each IR can be used to calculate action and phase inside that region. This extra information is used to independently estimate errors at each triplet or specifically the magnetic kick errors $\Delta x^{\prime}$ and $\Delta y^{\prime}$.

The magnetic kick errors $\Delta x^{\prime}$ and $\Delta y^{\prime}$ are functions of the horizontal and vertical beam position $x_{0}$ and $y_{0}$. These two last variables are not independent. There is a relation between them that depends on the amount of coupling present in the ring, and that is easily determined experimentally. This means that $\Delta x^{\prime}$ and $\Delta y^{\prime}$ can be seen as quantities that depend on only one variable, either, $x_{0}$ or $y_{0}$.

Fig. 5.2 is a typical graph showing $\Delta x^{\prime}$ (line with long dashes), $\Delta y^{\prime}$ (solid line) and $x_{0}$ as a function of $y_{0}$ at the right triplet of IR 2, for a set of four difference orbits taken after changing the strength of the vertical dipole corrector bo7-tv13 to $-0.1 \mathrm{mrad},-0.05 \mathrm{mrad}, 0.05 \mathrm{mrad}$ and $0.1 \mathrm{mrad}$ (each point in the graph corresponds to a different dipole corrector strength).

As can be seen in Fig. 5.2, the behavior of $\Delta x^{\prime}$ and $\Delta y^{\prime}$ as a function of $x_{0}$ is almost linear, pointing to the fact that the contribution of nonlinear errors to the action and phase jump at the right triplet of IR 2 is very small compared with the contribution from linear errors. 


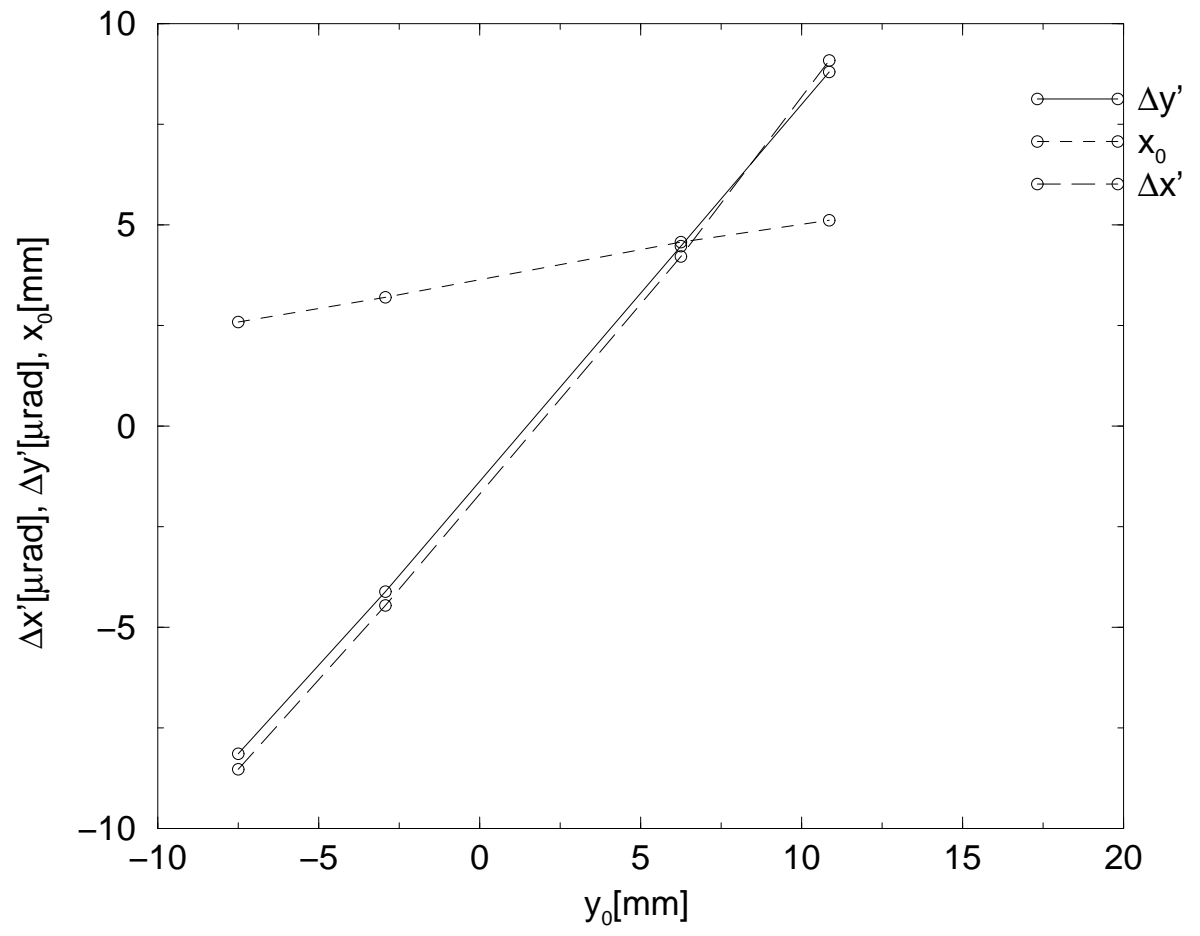

Figure 5.2: Relation between magnetic kicks and beam position. This data was taken in the right triplet of IR2 of the Blue ring. The different dots in all 3 lines correspond to different strength settings of the dipole corrector bo7-tv13. 
According to Eq. 1.56, only 2 coefficients $\left(A_{1}, B_{1}\right)$ have to be determined from the slopes of the curves of Fig. 5.2. Studies after the analysis done in Section 5.2 show that coefficients $B_{1}$ has to be different in the horizontal and the vertical plane (see Section B.3) due to the fact that more than one magnet is contributing to the magnetic error $\Delta x^{\prime}$ and $\Delta y^{\prime}$.

The correct equation correspond to the linear part of Eq. B.33 which is:

$$
\begin{aligned}
& \Delta x^{\prime}=A_{1}^{e q} y_{0}-B_{1}^{x} x_{0} \\
& \Delta y^{\prime}=A_{1}^{e q} x_{0}+B_{1}^{y} y_{0}
\end{aligned}
$$

There are now 3 unknown coefficients: $A_{1}, B_{1}^{x} B_{1}^{y}$ and there are only 2 curves that provide information about those coefficients. The third curve just gives the relation between $x_{0}$ and $y_{0}$. It is then necessary to do a similar experiment in the vertical plane that will provide the additional slope needed to find the 3 unknown coefficients.

Graphs obtained by changing the strength of the horizontal corrector (subscript a) and graphs obtained by changing the strength of the vertical corrector (subscript b) lead to the following system of equations:

$$
\begin{aligned}
& y_{0}=m_{a} x_{0}+b_{a} \\
& x_{0}=m_{b} y_{0}+b_{b}
\end{aligned}
$$


and

$$
\begin{aligned}
& \Delta x^{\prime}=c_{1}^{x a} x_{0}+\text { constant } \\
& \Delta y^{\prime}=c_{1}^{y a} x_{0}+\text { constant } \\
& \Delta x^{\prime}=c_{1}^{x b} y_{0}+\text { constant } \\
& \Delta y^{\prime}=c_{1}^{y b} y_{0}+\text { constant }
\end{aligned}
$$

here, $m_{a}$ and $m_{b}, c_{1}^{x_{a}}, c_{1}^{x b}, c_{1}^{y a}$ and $c_{1}^{y b}$ are constants that are directly found from graphs like Fig. 5.2. Putting together Equations 5.21, 5.22 and 5.23, it is possible to relate the unknown coefficients, $A_{1}^{e q}, B_{1}^{x}$ and $B_{1}^{y}$ with the constants extracted from the kick vs position graphs in the following way:

$$
\begin{aligned}
c_{1}^{x a} & =m_{a} A_{1}^{e q}-B_{1}^{x} \\
c_{1}^{y a} & =A_{1}^{e q}+m_{a} B_{1}^{y} \\
c_{1}^{x b} & =A_{1}^{e q}-m_{b} B_{1}^{x} \\
c_{1}^{y b} & =m_{b} A_{1}^{e q}+B_{1}^{y}
\end{aligned}
$$

Only 3 equations are needed from the previous set of equations to solve for the 3 unknown coefficients. Standard methods are applied to the 3 selected equations to obtain: 


$$
\begin{aligned}
A_{1}^{e q} & =\frac{c_{1}^{y a}-c_{1}^{y b} m_{a}}{1-m_{a} m_{b}} \\
B_{1}^{x} & =\frac{c_{1}^{x a}-c_{1}^{y a} m_{a}+c_{1}^{y b} m_{a}{ }^{2}-c_{1}^{x a} m_{a} m_{b}}{-1+m_{a} m_{b}} \\
B_{1}^{y} & =\frac{c_{1}^{y b}-c_{1}^{y a} m_{b}}{1-m_{a} m_{b}}
\end{aligned}
$$

Eq. 5.25 requires at least 3 different orbits.

There is an alternate way of finding $A_{1}, B_{1}^{x}$ and $B_{1}^{y}$ with only 2 orbits. In order to find the corresponding relation we write:

$$
\begin{aligned}
& \Delta x_{1}^{\prime}=-B_{1}^{x} x_{1}+A_{1}^{e q} y_{1} \\
& \Delta y_{1}^{\prime}=A_{1}^{e q} x_{1}+B_{1}{ }^{y} y_{1}
\end{aligned}
$$

and similarly for the second orbit:

$$
\begin{aligned}
& \Delta x_{2}^{\prime}=-B_{1}^{x} x_{2}+A_{1}^{e q} y_{2} \\
& \Delta y_{2}^{\prime}=A_{1}{ }^{e q} x_{2}+B_{1}^{y} y_{2}
\end{aligned}
$$

Choosing only 3 of these equations it is possible to find the linear coefficients with the relations:

$$
B_{1}^{x}=\frac{y_{1} \Delta x_{2}^{\prime}-y_{2} \Delta x_{1}^{\prime}}{-\left(x_{2} y_{1}\right)+x_{1} y_{2}}
$$




$$
\begin{aligned}
B_{1}^{y} & =\frac{x_{1}^{2} \Delta x_{2}^{\prime}+x_{2} y_{1} \Delta y_{1}^{\prime}-x_{1}\left(x_{2} \Delta x_{1}^{\prime}+y_{2} \Delta y_{1}^{\prime}\right)}{y_{1}\left(x_{2} y_{1}-x_{1} y_{2}\right)} \\
A_{1}^{e q} & =\frac{x_{1} \Delta x_{2}^{\prime}-x_{2} \Delta x_{1}^{\prime}}{-\left(x_{2} y_{1}\right)+x_{1} y_{2}}
\end{aligned}
$$

The 2 orbits used to evaluate these coefficients should be independent of each other. That means that the orbits should be excited with different dipole correctors and with a difference of phase advance different to $\pi$ or any multiple of $\pi$.

Since the orbits must be independent of each other they cannot fulfill the phase advance requirement $(\Delta \psi \approx \pi / 2)$ simultaneously. This has consequences in the determination of the beam position at the skew quadrupole corrector with Eq. 3.9. Eq. 3.9 is an approximation that relies on the fact that the phase advance from the nearest BPM to the skew quadrupole corrector is very small. The importance of this small phase advance is determined by the difference phase advance between the dipole corrector used to excite the betatron oscillation and the interaction region. The farther this phase advance is from 90 degrees the bigger is the effect on the difference phase advance between skew corrector and the nearest BPM to the skew quadrupole corrector. If accurate results are required this difference must be taken into account in the calculations. 


\subsubsection{Results for Proton Experiments Different Horizontal and Vertical Gradients}

If different equivalent gradients are assumed in the horizontal and the vertical plane it is necessary to use difference orbits produced by a horizontal and a vertical dipole corrector to obtain the linear errors. Table 5.5 shows several pair of dipole correctors that were used to obtain the difference orbits of protons required to evaluate the errors at the left triplet of IR8.

Each pair produces two graphs like Fig. 5.2 which usually are straight lines. The slopes of the straight lines are then used in Eq. 5.25 to find the corresponding errors.

Several pairs of dipole correctors are used in order to evaluate the uncertainty of the measurements that are mainly due to the difference phase advance $\Delta \psi^{c i}$, as discussed earlier.

The same procedure is followed for most of the RHIC triplets leading to Table 5.6. It is important to mention that most of the triplets have small skew errors as expected, since the skew quadrupole correctors were on when the experiments were done. Another important comment is that difference orbits produced with the same pair of dipole correctors are used to evaluate errors in more than one IR.

Table 5.7 shows the results obtained from the analysis of difference orbits in the Yellow ring. Only two pairs of dipole correctors were used in each case. The uncertainty was calculated as half the difference between the mea- 


\begin{tabular}{|c|c|c|c|c|}
\hline Corrector & Triplet & $\begin{array}{c}A_{1} \\
\left(10^{-3} 1 / \mathrm{m}\right)\end{array}$ & $\begin{array}{c}B_{1}^{x} \\
\left(10^{-3} 1 / \mathrm{m}\right)\end{array}$ & $\begin{array}{c}B_{1}^{y} \\
\left(10^{-3} 1 / \mathrm{m}\right)\end{array}$ \\
\hline bo2-th14 & 7 & -0.006 & 0.655 & 0.211 \\
bo6-tv15 & & & & \\
bo3-th10 & 7 & -0.003 & 0.641 & 0.213 \\
bo6-tv15 & & & & \\
bo3-th12 & 7 & -0.114 & 0.788 & 0.206 \\
bo6-tv15 & & & & \\
Average & 7 & -0.067 & 0.694 & 0.210 \\
Error & & \pm 0.020 & \pm 0.050 & \pm 0.002 \\
\hline
\end{tabular}

Table 5.5: Sample of error analysis done with protons orbits at the left triplet of IR8 in the Blue ring.

surements obtained for each pair of correctors. Again, the skew quadrupole errors were small, as expected, since the skew quadrupole correctors were on when the difference orbits were taken. The errors shown on Table 5.6 and Table 5.7 put a limitation in the minimum skew error that can be measured at around $10^{-4} 1 / \mathrm{m}$. Many of the skew errors fall around or maybe below this value, as was expected, since the local skew correctors were on when the data to produce Table 5.6 were taken. The magnitude of the error associated with each measurement depends more on the dipole corrector that is chosen to produce the betatron oscillations, than on the BPM measurements. This is an indication that the measurements are sensitive to the difference of phase advance between the dipole corrector and the IR. Even though the orbits used to find the errors at a particular IR were chosen with optimal phase advance 


\begin{tabular}{|c|r|r|r|}
\hline Triplet & \multicolumn{1}{|c|}{$\begin{array}{c}A_{1} \\
\left(10^{-3} 1 / \mathrm{m}\right)\end{array}$} & \multicolumn{1}{c|}{\begin{tabular}{c}
\multicolumn{1}{c|}{$B_{1}^{x}$} \\
\end{tabular}} & \multicolumn{1}{c|}{$\left.B_{1}^{y} 1 / \mathrm{m}\right)$} \\
\hline 1 & $-0.866 \pm 0.152$ & $-0.655 \pm 0.013$ & \multicolumn{1}{c|}{$4.674 \pm 1.805$} \\
2 & $-0.270 \pm 0.001$ & $-3.991 \pm 0.000$ & $0.961 \pm 0.005$ \\
7 & $-0.067 \pm 0.024$ & $0.690 \pm 0.040$ & $0.200 \pm 0.002$ \\
8 & $0.162 \pm 0.031$ & $-0.280 \pm 0.030$ & $-0.355 \pm 0.003$ \\
9 & $-0.048 \pm 0.007$ & $-0.235 \pm 0.009$ & $-0.850 \pm 0.010$ \\
10 & $0.206 \pm 0.027$ & $1.490 \pm 0.019$ & $0.715 \pm 0.000$ \\
11 & $0.076 \pm 0.031$ & $-6.676 \pm 1.128$ & $4.618 \pm 0.003$ \\
12 & $-0.316 \pm 0.073$ & $4.766 \pm 0.307$ & $-6.398 \pm 0.014$ \\
\hline
\end{tabular}

Table 5.6: Linear errors in the Blue ring triplets. Proton orbits were used in this analysis.

\begin{tabular}{|c|c|c|c|}
\hline Triplet & $\begin{array}{c}A_{1} \\
\left(10^{-3} 1 / m\right)\end{array}$ & $\begin{array}{c}B_{1}^{x} \\
\left(10^{-3} 1 / m\right)\end{array}$ & $\begin{array}{c}B_{1}^{y} \\
\left(10^{-3} 1 / m\right)\end{array}$ \\
\hline 1 & $0.016 \pm 0.014$ & $1.889 \pm 0.054$ & $-1.21 \pm 0.001$ \\
\hline 2 & & -1.2 & 0.861 \\
\hline 9 & $65 \pm 0.03$ & $1.248 \pm 0.0$ & $-1.007 \pm 0.004$ \\
\hline 10 & $0.147 \pm 0.044$ & $-2.675 \pm 2.120$ & $1.030 \pm 0.003$ \\
\hline
\end{tabular}

Table 5.7: Linear errors in Yellow Ring Triplets. Proton orbits were used in this analysis.

in one of the planes, it was not always possible to meet the same condition in the other plane. It is possible to have complete control over this problem if a horizontal and vertical dipole corrector are used simultaneously to produce the betatron oscillation.

Another possible source of error is the slight difference in tunes between the model used to do the analysis and the real tune of the machine. This difference creates a slight slope in the graphs of phase vs $s$ in the arcs. A re-tuning of the lattice model should reduce this tilt in the phase. Doing all 


\begin{tabular}{|c|r|r|c|}
\hline Triplet & \multicolumn{1}{|c|}{$\begin{array}{c}A_{1} \\
\left(10^{-3} 1 / \mathrm{m}\right)\end{array}$} & \multicolumn{1}{c|}{$\begin{array}{c}B_{1} \\
\left(10^{-3} 1 / \mathrm{m}\right)\end{array}$} & Correctors Plane \\
\hline 1 & $-1.085 \pm 0.005$ & $-1.770 \pm 0.020$ & Vertical \\
2 & 1 & $0.775 \pm 0.005$ & Vertical \\
7 & $0.005 \pm 0.009$ & $0.680 \pm 0.035$ & Horizontal \\
8 & $0.083 \pm 0.104$ & $-0.287 \pm 0.029$ & Horizontal \\
9 & $0.018 \pm 0.001$ & $-0.840 \pm 0.015$ & Vertical \\
10 & $0.120 \pm 0.050$ & $1.290 \pm 0.010$ & Horizontal \\
11 & $-2.160 \pm 0.830$ & $-6.270 \pm 0.707$ & Horizontal \\
12 & $1.450 \pm 0.290$ & $4.480 \pm 0.270$ & Horizontal \\
\hline
\end{tabular}

Table 5.8: Error analysis in Blue ring assuming that the gradients are the same in both planes

previous corrections it is not unreasonable to expect measurements of the skew errors with accuracies at the $10^{-5} 1 / \mathrm{m}$ level.

\section{Same Horizontal and Vertical Gradients}

If it is assumed that the horizontal and vertical equivalent gradients are the same (as it was done when the action and phase analysis was applied to RHIC orbits for the first time [28]) it is then possible to find the linear coefficients with only one orbit produced by either a horizontal dipole corrector, or by a vertical dipole corrector. This assumption seems to work well when the betatron oscillation in the plane opposite to the exciting dipole corrector is very small compared to the betatron oscillation in the plane of the exciting dipole corrector. Table 5.8 shows that indeed the skew quadrupole errors calculated in this way are very close to the ones reported in Table 5.6, but with higher uncertainties. Gradient errors calculated with orbits produced with a horizontal dipole corrector can be compared with the horizontal gradients 
found previously in Table 5.6. The same is true for the vertical plane. This analysis it is interesting because allows to approximate 2 linear coefficients with only one difference orbit. It is shown here also because it was the first approach used to analyze the data.

\subsubsection{Results for Gold Ion Experiments}

Dipole corrector strengths were varied only twice for gold ions, but for each strength several orbits were taken. Two different strengths are enough to calculate linear errors, assuming that nonlinear errors are insignificant when

compared to linear errors. As an example, the graphs of magnetic kicks for the experiment performed with yi10-th13 are shown in Fig. 5.3. Notice that there are two group of points in each curve of Fig. 5.3. These points correspond to different orbits taken with the same strength, and they give an idea of the uncertainty in the determination of the magnetic kicks.

In order to apply Eq. 5.25 it is then neccesary to have a similar graph to Fig. 5.3, but this time the closed orbit should be shifted by a vertical dipole corrector. Such a graph is shown in Fig. 5.4, where the vertical dipole corrector yi10-tv12 is used. Curves of $\Delta y^{\prime}$ vs $y_{0}$ and $x_{0}$ vs $y_{0}$ are repeatable, since the dots in each group are very close to each other. This is not the case for $\Delta x^{\prime}$ vs $y_{0}$, where the uncertainty is very large. It is possible to track the reason why the uncertainty is so large by looking at the orbits behind the points in Fig. 5.4. One of such orbits is shown in Fig. 5.5. It is clear that the fit is not as good as it is in Fig. 3.1 which is also obvious in the actions and phases 


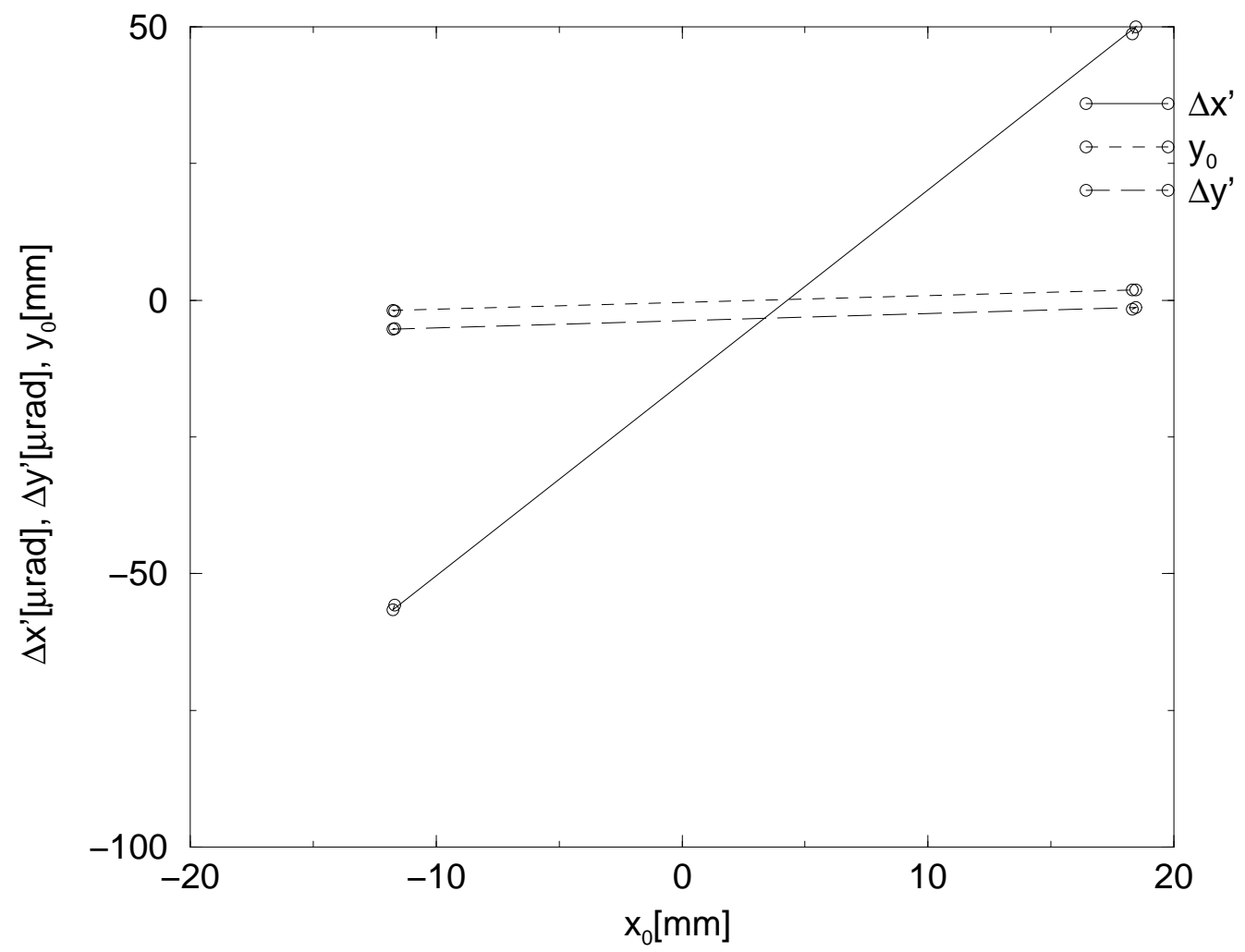

Figure 5.3: Relation between Magnetic kicks and Beam Position for Gold ions in triplet 7 of the Yellow ring when dipole corrector yi10-th13 is used to shift the closed orbit. 


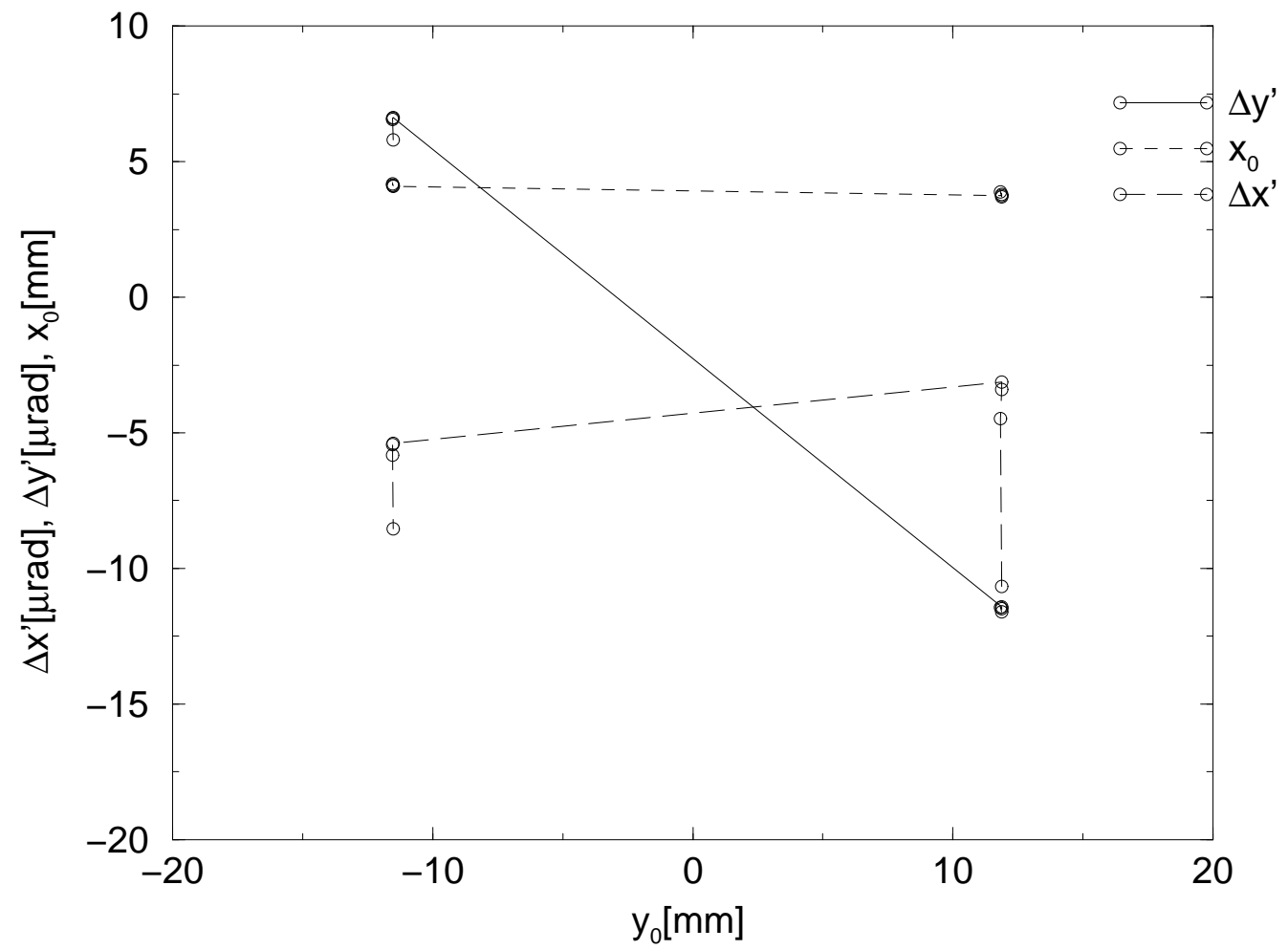

Figure 5.4: Relation between Magnetic kicks and Beam Position for Gold ions in triplet 7 of the Yellow ring when dipole corrector yi10-tv12 is used to shift the closed orbit. 


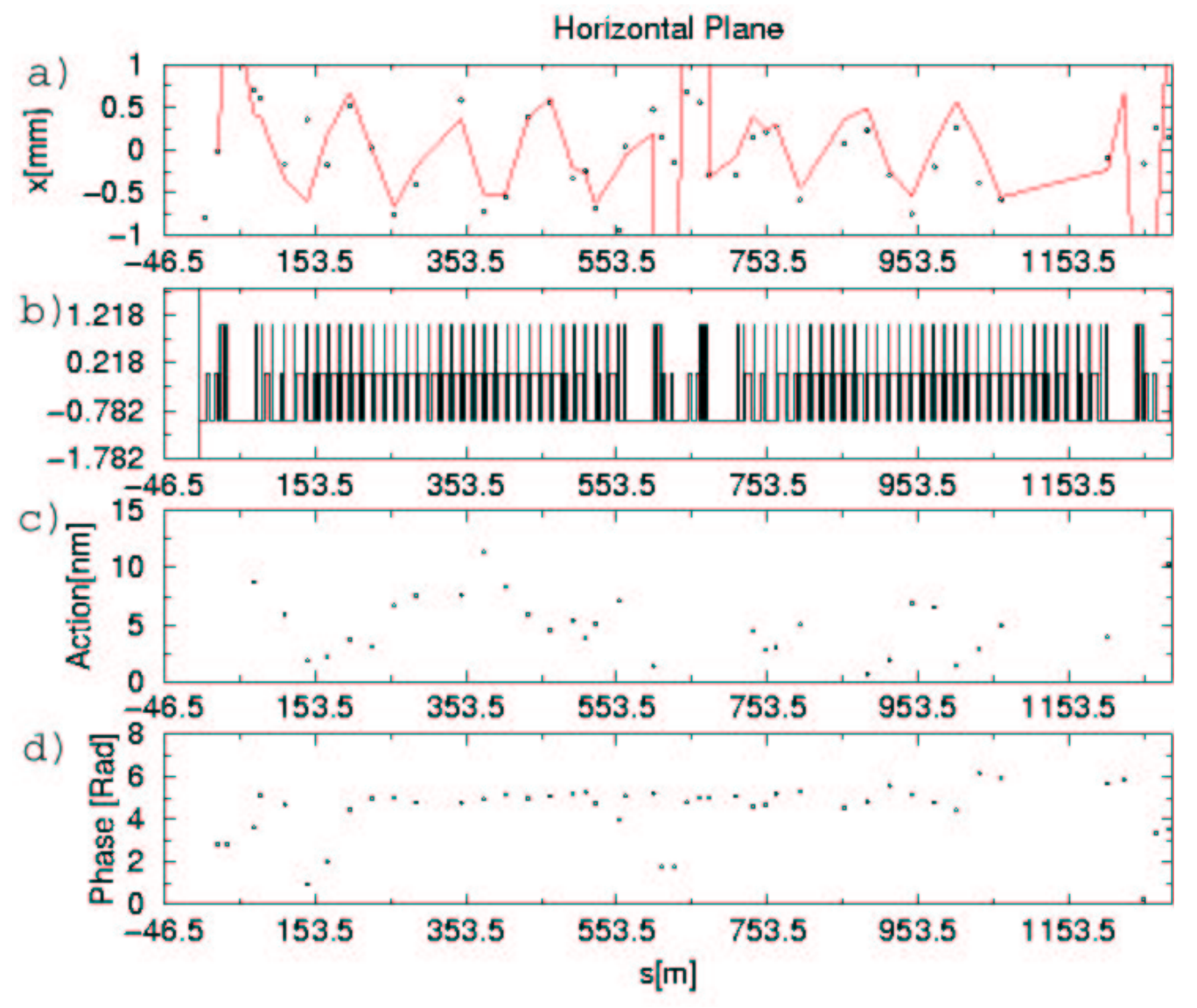

Figure 5.5: a) Yellow ring difference orbit. b) Lattice representation of RHIC. c) Action analysis of orbit in a). d) Phase analysis of the orbit in a). The model (solid line in window a)) follows irregularly the BPM data and action and phase can hardly be considered as constant values. 
having large variations in the arcs.

The fast pace of RHIC commissioning during the year 2001 only permitted difference orbits to be taken with gold ions in the Yellow ring.

Table 5.9 shows the errors that were obtained in some of the Yellow triplets. When not enough useful orbits were available to do the analysis with Eq. 5.25, Eq. 5.17 were used to extract $A_{1}$ and $B_{1}$ from a single orbit. According to studies in the previous section, this formula seems to be a good approximation to find 2 of the 3 linear errors.

\begin{tabular}{|c|c|c|c|c|}
\hline Corrector & Triplet & $\begin{array}{c}A_{1} \\
\left(10^{-3} 1 / \mathrm{m}\right)\end{array}$ & $\begin{array}{c}B_{1}^{x} \\
\left(10^{-3} 1 / \mathrm{m}\right)\end{array}$ & $\begin{array}{c}B_{1}^{y} \\
10^{-3} 1 / \mathrm{m}\end{array}$ \\
\hline yi10-tv14 & 3 & 1.55 & & -2.82 \\
yi10-th13 \& yi10-tv14 & 4 & 0.19 & -4.40 & -2.05 \\
yi10-tv12 & 5 & -1.32 & & 6.34 \\
yi10-th11 \& yi10-tv12 & 6 & -3.15 & 3.30 & 6.03 \\
yi10-th13 & 7 & 0.55 & -3.44 & \\
yi10-th13 & 8 & -0.98 & 5.07 & \\
yi10-th13 & 11 & 0.68 & -1.90 & \\
yi10-th13 & 12 & -0.63 & 2.77 & \\
\hline
\end{tabular}

Table 5.9: Linear errors in some of the Yellow triplets obtained from gold ion difference orbits. When only difference orbits created with one corrector are available for a specific IR Eq. 5.17 is used instead of Eq. 5.25

\subsubsection{Tune correction of the lattice model}

Graphs of phase and action show that there is a slight tilt of these two variables in the arcs of the accelerator where otherwise phase and action should be constant (see Fig. 5.6). The slight tilt is due to a difference between the real tunes of the machine and the tunes of the model. Hence, it is necessary 


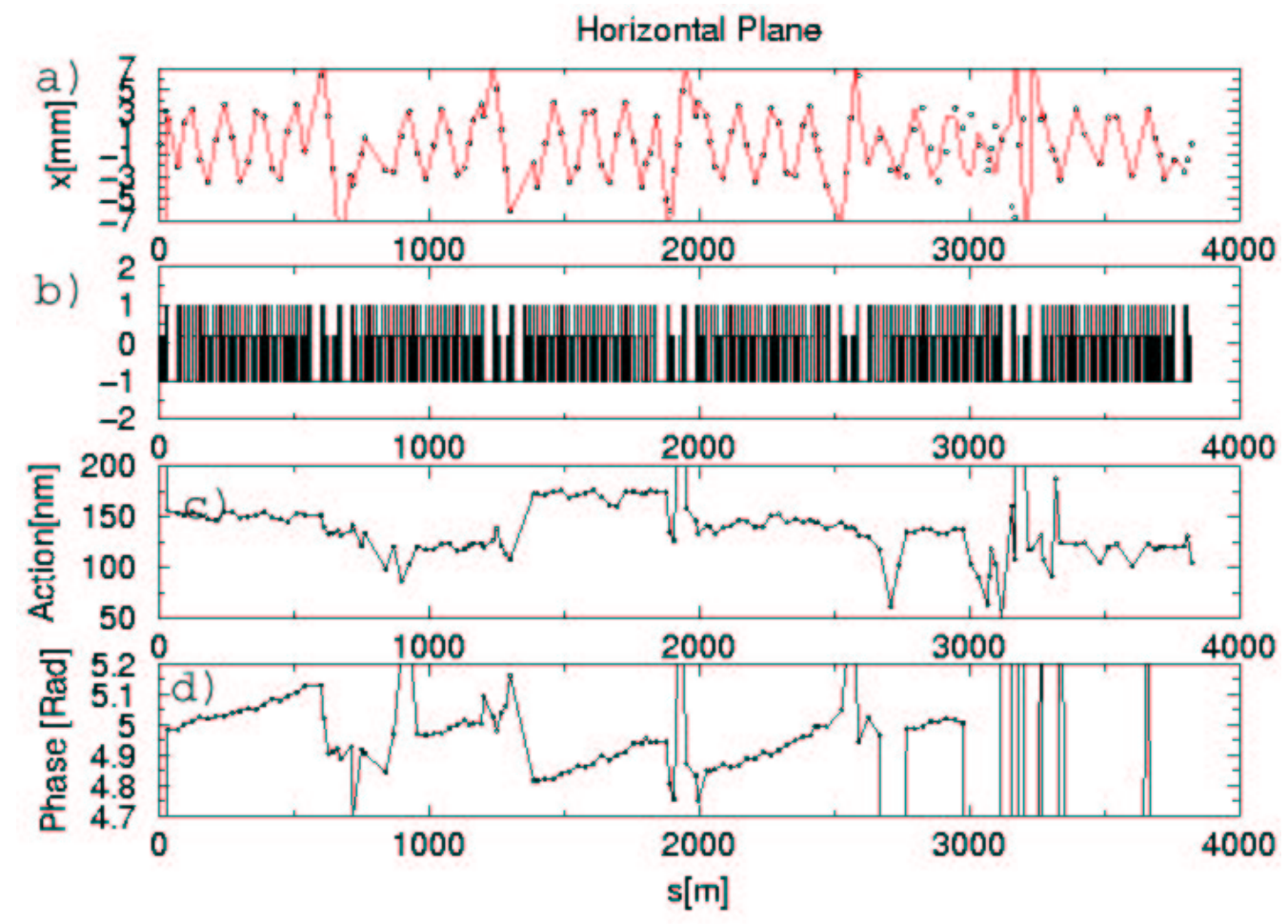

Figure 5.6: a) Difference orbit taken during the RHIC 2001 run. b) Lattice representation of RHIC. c) Action analysis of the orbit in a). d) Phase analysis of the orbit in a). The tilt on the curves of phase is due to difference between the real tunes of the machine and the tunes of the model. 
to re-tuned the model until the tilt disappear.

All the analysis done in the previous section were repeated with a re-tuned model with results that can be seen in Table 5.10. There are no significant changes in the linear coefficients when they are compared with the corresponding values in Table 5.6. These results seem to indicate that small variations in tune don't have significant effects in the determination of the linear errors.

\begin{tabular}{|c|r|r|r|}
\hline Triplet & \multicolumn{1}{|c|}{$\begin{array}{c}A_{1} \\
\left(10^{-3} 1 / \mathrm{m}\right)\end{array}$} & \multicolumn{1}{c|}{$\begin{array}{c}B_{1}^{x} \\
\left(10^{-3} 1 / \mathrm{m}\right)\end{array}$} & \multicolumn{1}{c|}{$\begin{array}{c}B_{1}^{y} \\
\left(10^{-3} 1 / \mathrm{m}\right)\end{array}$} \\
\hline 7 & $-0.067 \pm 0.024$ & $0.870 \pm 0.020$ & $0.210 \pm 0.001$ \\
8 & $0.199 \pm 0.025$ & $0.028 \pm 0.027$ & $-0.350 \pm 0.003$ \\
9 & $-0.048 \pm 0.007$ & $-0.230 \pm 0.009$ & $-0.850 \pm 0.010$ \\
10 & $0.206 \pm 0.027$ & $1.496 \pm 0.019$ & $0.716 \pm 0.001$ \\
11 & $0.077 \pm 0.031$ & $-6.680 \pm 1.130$ & $4.610 \pm 0.003$ \\
12 & $-0.320 \pm 0.070$ & $4.770 \pm 0.310$ & $-6.399 \pm 0.014$ \\
1 & $-0.870 \pm 0.150$ & $-0.660 \pm 0.013$ & $4.670 \pm 1.800$ \\
2 & $-0.270 \pm 0.001$ & $-3.990 \pm 0.000$ & $0.960 \pm 0.005$ \\
\hline
\end{tabular}

Table 5.10: Linear Errors from difference orbits in the Blue ring. The model has been re-tuned from $\mathrm{Qx}=28.22$ to $\mathrm{Qx}=28.12$.

\subsection{Comparison between RHIC Run 2000 and RHIC Run 2001}

Table 5.11 shows the relations between the different coupling measurements made during RHIC Run 2000 and RHIC Run 2001.

The first column shows the values to which the skew quadrupole correctors were set in the corresponding triplets during RHIC Run 2001. The second column shows the measurement of the skew errors made during RHIC Run 


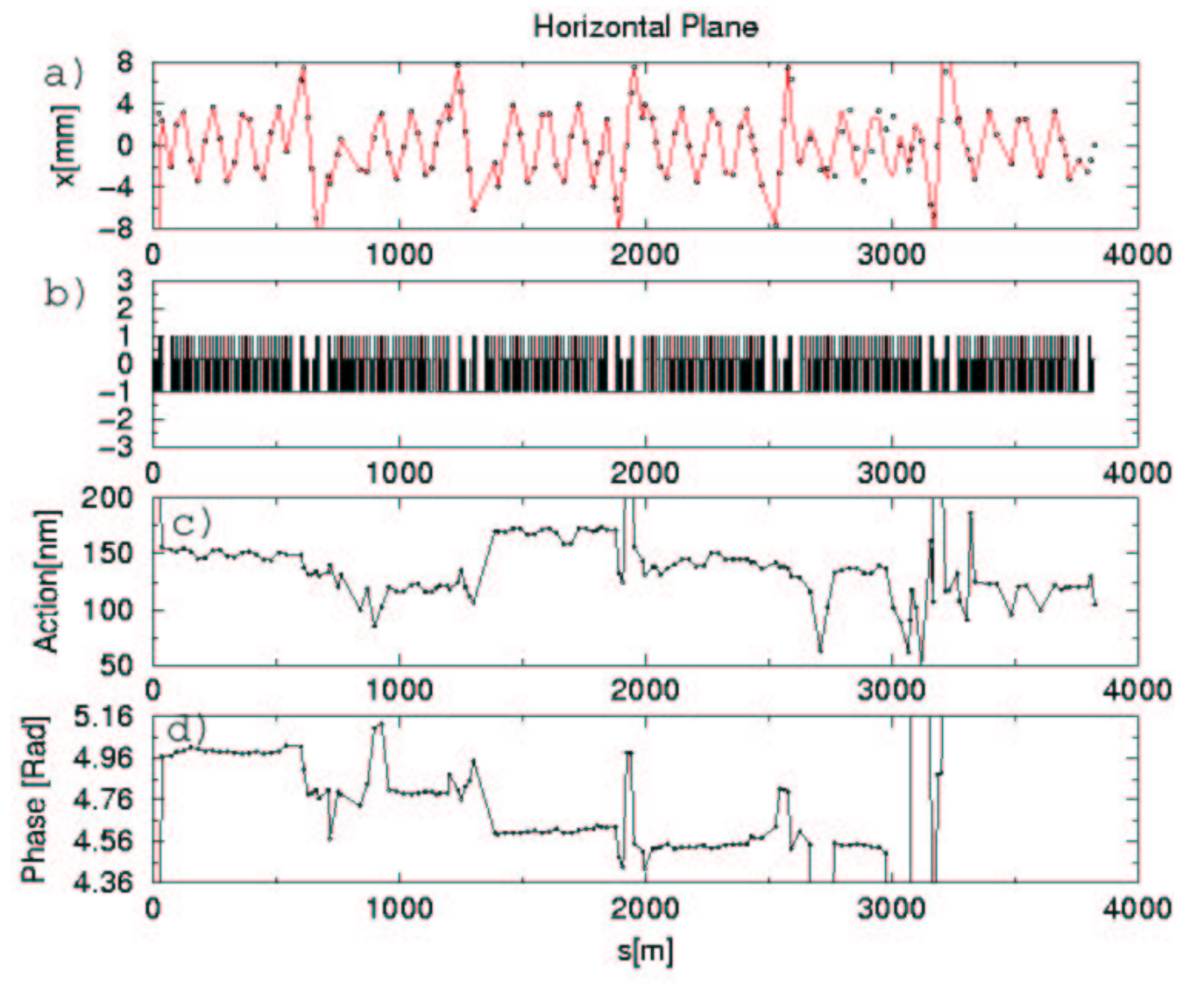

Figure 5.7: a) Difference orbit taken during the RHIC 2001 run. b) Lattice representation of RHIC. c) Action analysis of the orbit in a). d) Phase analysis of the orbit in a). The tilt on the curves of phase gets significantly reduced when the horizontal tune of the model is changed from 28.22 to 28.12 


\begin{tabular}{|c|c|c|c|c|c|c|}
\hline Triplet & Corrector & $\begin{array}{c}\text { Residual } \\
(2001)\end{array}$ & $\begin{array}{c}\text { Total } \\
\text { Triplet }\end{array}$ & Total IR & $\begin{array}{c}\text { Orbit Bump } \\
(2000)\end{array}$ & $\begin{array}{c}\text { Action- } \\
\text { Phase Jump }\end{array}$ \\
\hline 7 & -0.8 & 0 & -0.8 & \multirow{2}{*}{0.6} & -0.84 & \multirow{2}{*}{0.67} \\
\hline 8 & 1.3 & 0.1 & 1.4 & & 1.32 & \\
\hline 9 & 0.35 & -0.035 & 0.32 & \multirow{2}{*}{1.1} & & \multirow{2}{*}{1} \\
\hline 10 & 0.65 & 0.13 & 0.78 & & & \\
\hline 1 & 1 & -1.1 & -0.1 & \multirow{2}{*}{0.9} & -0.22 & \multirow{2}{*}{0.99} \\
\hline 2 & 0 & 1 & 1 & & 1.23 & \\
\hline
\end{tabular}

Table 5.11: Comparison between skew error measurements done in the Blue ring during the run 2000 vs the corresponding measurements done during the run 2001 (All values are given in $10^{-3} 1 / \mathrm{m}$ ).

2001 (see Table 5.8) with the action-phase jump method. This is naturally called the residual error since this is the error still present after turning on the corrector. The total triplet column corresponds to the sum of the previous two columns. The Total IR column is the sum of the left and right triplets of each IR from the previous column.

The Total Triplet column and the Total IR column are calculated with the purpose of making comparison with measurements made during Run 2000 when the correctors were off.

The Orbit Bump column shows the measurements of the skew errors made during Run 2000 with the Orbit Bump Method (see Table D.1). A good agreement between this last column and the total triplet column can be verified on the table. The other two columns to be compared are the action-phase jump column and the Total IR column. The action-phase jump column corresponds to the measurement made during RHIC Run 2000 with action-phase jump method (see Table 3.2). There is also good agreement in this case as can be 
verified on the table.

The agreement between the 2000 and the 2001 measurements are a little surprising since they were done with different lattices. However, the result is in agreement with the model predictions, as explained below.

The equivalent skew error depends on the individual gradients at each quadrupole of the triplet, and the equivalent skew errors also depend on the beta functions, as can be seen from Equations 1.54 and B.6. The lattice used during the RHIC 2000 Run differs with the corresponding lattice used in RHIC 2001 run only at IRs 6 and 10 . The beta function at the interaction point $\beta^{*}$ at these IRs was equal to 8 meters for the 2000 lattice, while the same quantity was equal to 3 meters for the 2001 lattice. In order to achieve the difference in $\beta^{*}$ the strengths of the quadrupoles at these IRs must be different. The difference in strengths, however, is as small as 2 percent (see Table 5.12).

The role of the beta functions in the determination of the equivalent skew quadrupole errors (see Table 5.13) was evaluated by direct calculation with the two different lattices assuming the roll angles were known . Calculated equivalent skew errors in IR10 and the left triplet of IR6 had a variation below 2 percent. Calculated equivalent skew errors in the right triplet of IR6 showed a relative large variation (more than 28 percent). 


\begin{tabular}{|c|c|c|c|}
\hline Triplet & Quadrupole & $\begin{array}{c}\mathrm{k} \text { Run } 2000 \\
1 / \mathrm{m}\end{array}$ & $\begin{array}{c}\mathrm{k} \text { Run } 2001 \\
1 / \mathrm{m}\end{array}$ \\
\hline \multirow{3}{*}{ Left IR6 } & Q3I6 & 0.1149 & 0.1165 \\
& Q2I6 & -0.1884 & -0.1900 \\
& Q1I6 & 0.0808 & 0.0828 \\
\hline \multirow{4}{*}{ Right IR66 } & Q1O6 & -0.0808 & -0.0828 \\
& Q2O6 & 0.1884 & 0.1900 \\
& Q3O6 & -0.1149 & -0.1165 \\
\hline \multirow{3}{*}{ Left IR10 } & Q3I10 & 0.1149 & 0.1165 \\
& Q2I10 & -0.1884 & -0.1900 \\
& Q1I10 & 0.0808 & 0.0828 \\
\hline \multirow{3}{*}{ Right IR10 } & Q1O10 & -0.0808 & -0.0828 \\
& Q2O10 & 0.1884 & 0.1900 \\
& Q3O10 & -0.1149 & -0.1165 \\
\hline
\end{tabular}

Table 5.12: Strengths of the Triplet Quadrupoles at IR6 and IR10. The third column corresponds to the nominal strength of the quadrupoles used in the Blue ring during the Gold run in 2000. The fourth column corresponds to the nominal strengths used in the Blue ring during the proton run in 2001. 


\begin{tabular}{|c|c|c|c|}
\hline Triplet & $\begin{array}{c}A_{1}\left(10^{-3} 1 / \mathrm{m}\right) \\
\text { Gold }(2000)\end{array}$ & $\begin{array}{c}A_{1}\left(10^{-3} 1 / \mathrm{m}\right) \\
\text { Proton }(2001)\end{array}$ & $\begin{array}{c}\text { Diff. } \\
\%\end{array}$ \\
\hline 1 & 0.0242 & 0.0243 & 0.246 \\
2 & 1.5250 & 1.5241 & -0.059 \\
3 & -0.6432 & -0.6438 & 0.085 \\
4 & 0.2931 & 0.2922 & -0.293 \\
5 & 0.6145 & 0.5956 & -3.170 \\
6 & 0.0374 & 0.0524 & 28.542 \\
7 & -1.5727 & -1.5731 & 0.025 \\
8 & 1.2007 & 1.2000 & -0.057 \\
9 & 0.4804 & 0.4819 & 0.317 \\
10 & 1.6918 & 1.7160 & 1.411 \\
11 & -0.1151 & -0.1163 & 1.055 \\
12 & -1.1693 & -1.1684 & -0.072 \\
\hline
\end{tabular}

Table 5.13: Calculated skew quadrupole errors from Eq. B.6 for the Gold lattice used during the RHIC 2000 run and the proton lattice used during the RHIC 2001 run. The roll angles were assumed to be twice the field angles measured before the magnets were installed in the tunnel (see Chapter 6). 


\section{Chapter 6}

\section{Skew Error Measurements and Roll Angle Measurements}

\subsection{Introduction}

As shown in Chapter 2, measurements based on orbit data taken during the RHIC 2000 run suggested that the sources of linear coupling in the machine were skew quadrupole errors produced by roll misalignments in the triplets at each IR. This hypothesis can be tested if the roll angles of the triplets are measured and their skew effect is calculated and compared with the measured skew errors. This is the main goal of this chapter where we will compare 2 different set of roll angle data with the corresponding skew errors obtained from the action and phase analysis. 


\subsection{Skew Error Measurements and $2 \phi$ Hypothesis}

The measured skew errors at the IRs suggest that the roll angles can be as big as several mrad. These values are extremely big since the accuracy of the alignment procedure was expected to be as low as tenths of mrad.

A possible answer for this problem can be found in the alignment procedure of the magnet. Before placing the magnets in the tunnel it is neccesary to measure what is called the field angle. The field angle is the angle between one of the transverse symmetry axis of the magnetic field and the corresponding transverse symmetry axis of the iron that supports the coil. Ideally this angle should be equal to zero but under real conditions this angle can be as big as several mrad for a typical RHIC magnet. It is then neccesary to measure the field angle for all the magnets and then rotate them in opposite direction to the field angle when they are placed in the tunnel. In this way, the effect of the magnetic field roll is minimized.

One possibility to explain the large angle implied by the skew error measurements is that the magnets were rotated in the same direction that the field angle giving rise to a total roll angle equal to twice the field angle (see Fig. 6.1). This hypothesis can be tested using the skew error measurements and comparing them with the values obtained from:

$$
A_{1}=\frac{\sum_{i=1}^{3}\left(-2 \frac{\phi_{i}}{f_{i}}\right) \sqrt{\beta_{x}^{i} \beta_{y}^{i}}}{\sqrt{\beta_{x}^{\text {Trip }} \beta_{y}^{\text {Trip }}}}
$$

Eq. 6.1 can be easily derived from Eq. B.6 and Eq. 1.54. The variables $f_{i}$, 


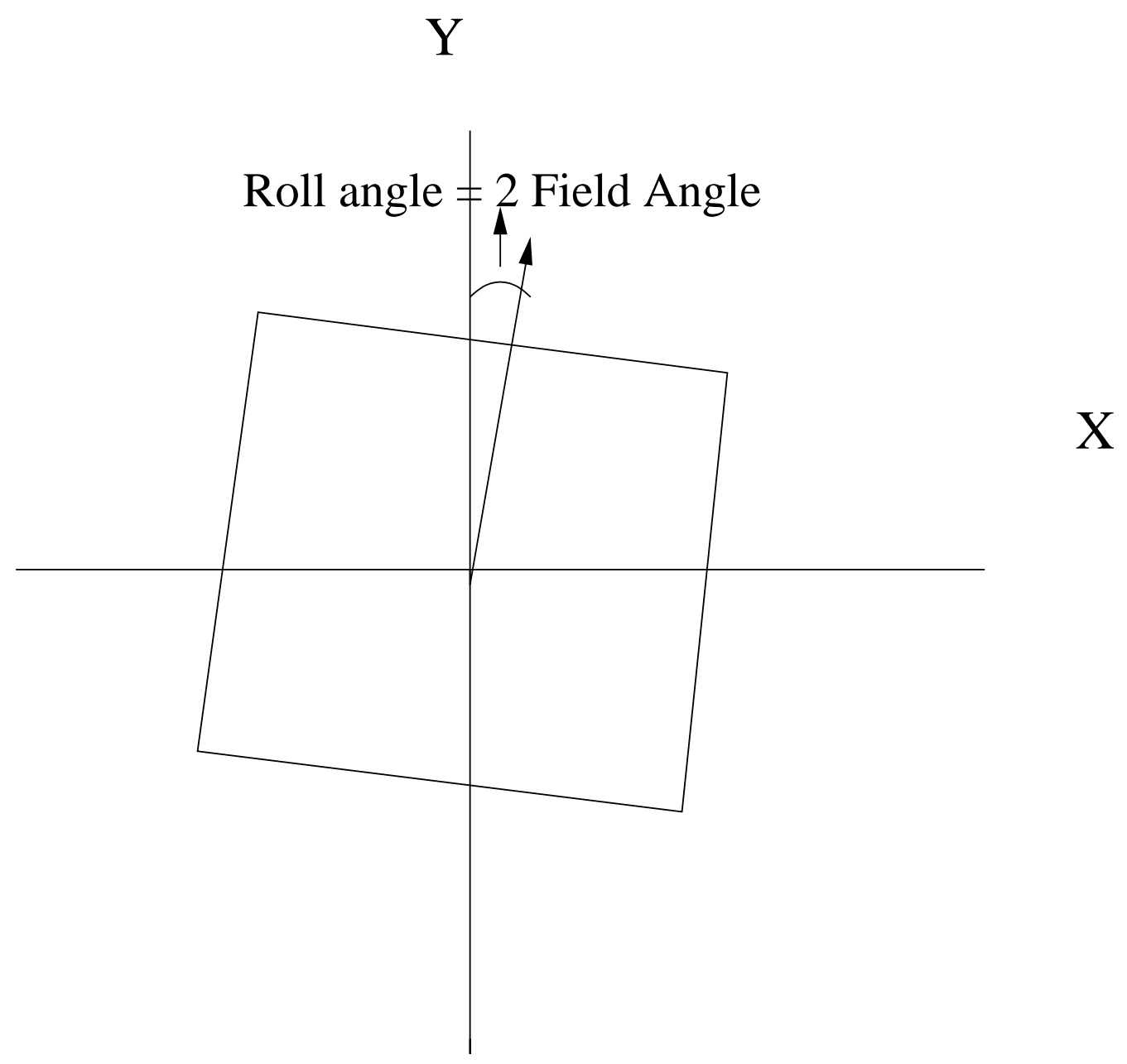

Figure 6.1: In order to cancel the field angle, the magnets are rotated opposite to the field angle when they are placed in the tunnel. It is possible that during this alignment procedure the magnets at the IR were rotated in the same direction that the field angle giving rise to a total roll angle equal to twice the field angle. 
$\phi_{i}, \beta_{x}^{i}, \beta_{y}^{i}$ in Eq. 6.1 correspond to the focal lengths, roll angles and beta functions (in both planes) of each individual quadrupoles that make compound

the triplet. $\beta_{x}^{\text {Trip }}, \beta_{y}^{\text {Trip }}$ are the beta functions at the place where the equivalent skew quadrupole error is calculated, in this case at the position of the skew quadrupole corrector.

According to Fig. 6.2 the skew errors obtained from the orbit measurements and the skew errors obtained from the database angles seem to have a good correlation at least for the stronger errors in the Blue ring.

The use of Eq. 6.1 requires some special considerations related with the way the field angles were measured and how they were placed in the ring. First, the field angle was always measured from the lead end of the magnet and second the triplet magnets are placed with their lead ends not always pointing in the same direction but as illustrated in Fig. 6.3. The position of the magnet lead end respect to the beam direction determines whether the magnet lead end is located CW (clockwise) or CCW (counter clockwise) (see Fig. 6.4). If the magnet lead end is located CW the database angles can be replaced in Eq. 6.1 with no change of sign but if the magnet lead end is located $\mathrm{CCW}$ the sign of the database angles must be changed.

\subsection{Roll Angle Measurements and $2 \phi$ Hypoth- esis}

During the 2002 shutdown period it was possible to directly measure the roll angles in some of the triplets and then prove the $2 \phi$ hypothesis directly. 


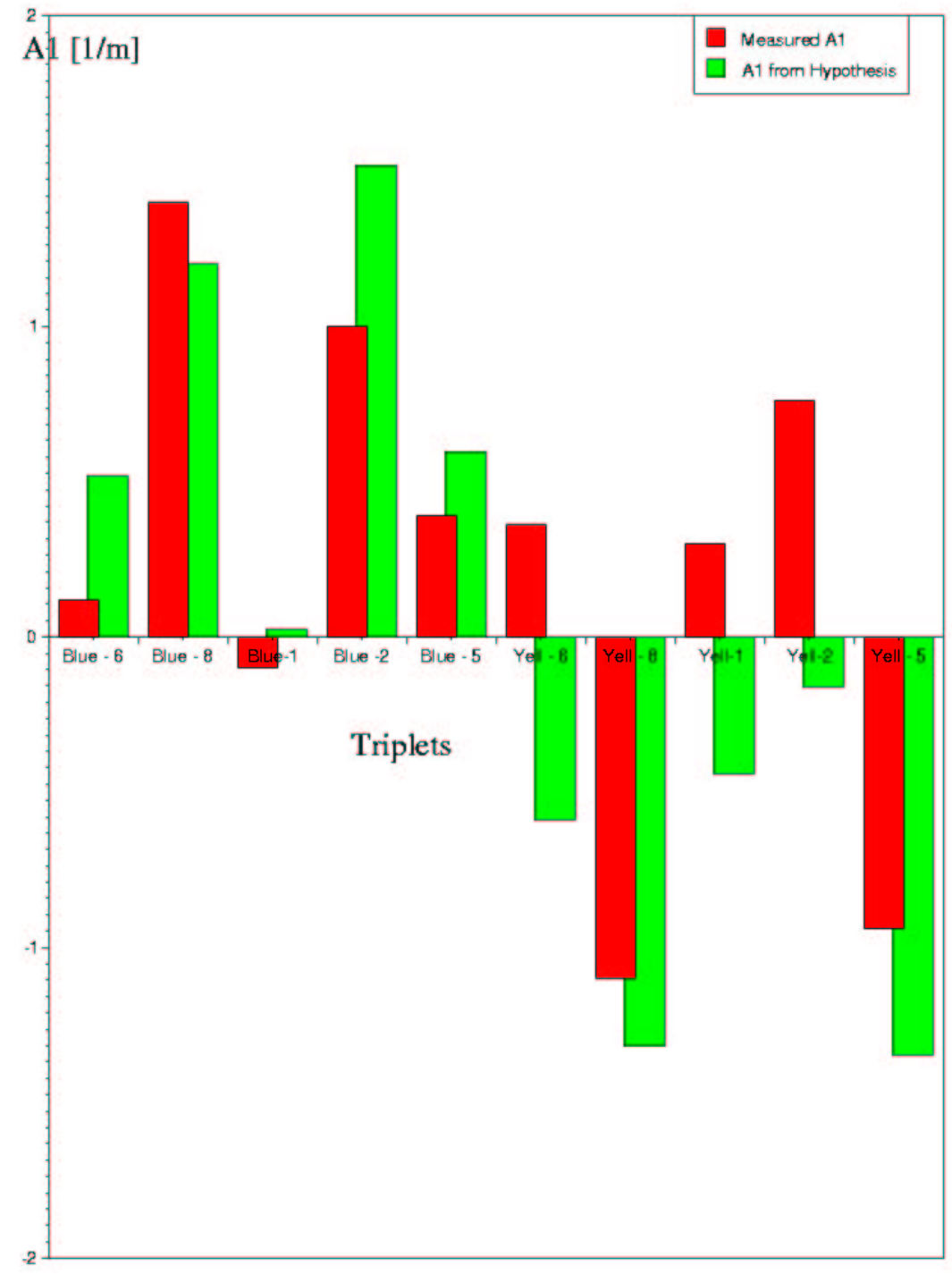

Figure 6.2: Skew errors from orbit measurements and skew errors calculated from the database field angles are compared. The measured errors were taken from Table 5.6 and Table D.1. 
a)

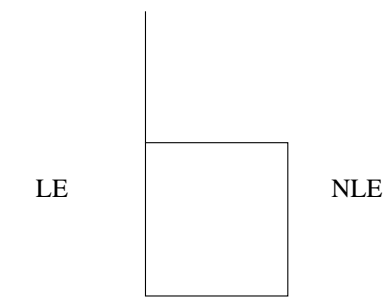

Left Triplet

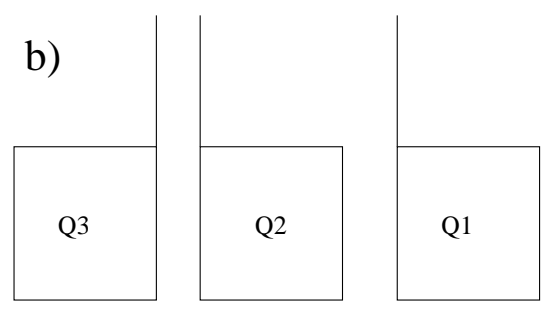

Right Triplet

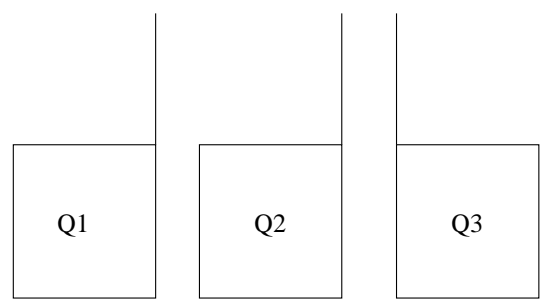

Figure 6.3: a) Representation of a RHIC IR quadrupole. b) Configuration of the RHIC IR quadrupoles at any IR. The quadrupoles in the IRs are placed in the tunnel with their lead ends not always facing the same direction.

The result of such comparisons can be seen on Fig. 6.5. The comparisons between the two sets of data show a relatively good agreement. Again, some conventions must be taken into account to do the comparisons. All the angles were measured from the Q1 no lead end (see Fig. 6.3 and Fig. 6.6). This means that the roll angles of the left triplet have the proper orientation to use Eq. 6.1 and no sign change is required. On the other hand, the sign of measured roll angles of the right triplet must be changed to use Eq. 6.1. The measured skew errors were also compared with the calculated skew errors using the measured roll angles (see Fig. 6.7). The positive results of Fig. 6.7 are a strong evidence that the major sources of coupling at the IRs are indeed roll quadrupoles.

Results of Fig. 6.2 and Fig. 6.7 are summarized in Fig. 6.8 where the 
a)

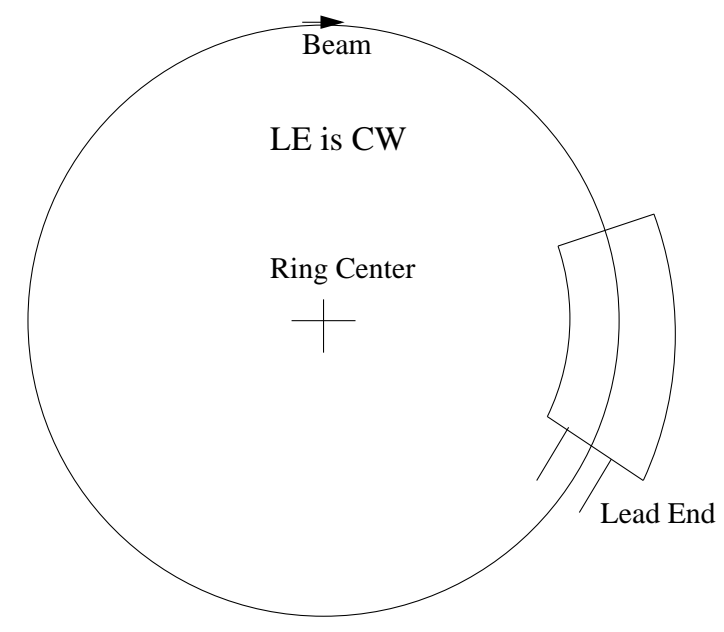

b)

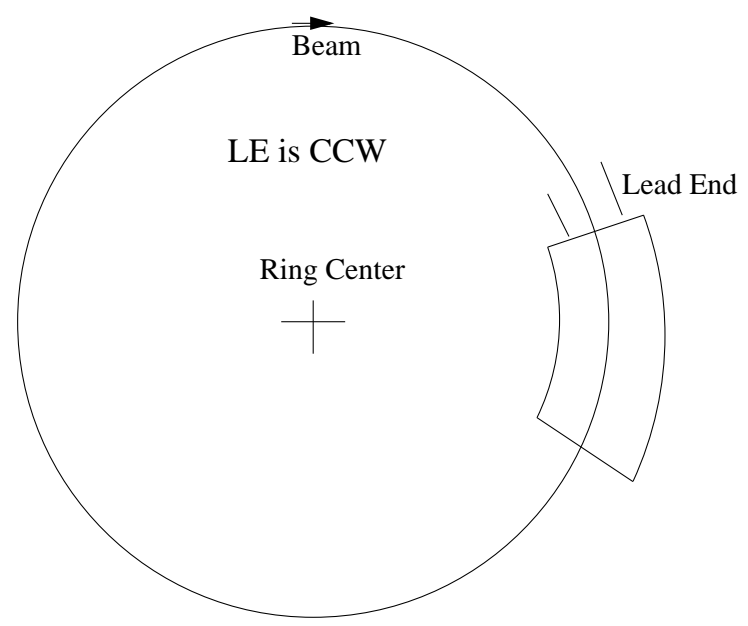

Figure 6.4: a) Magnet located in clockwise direction (CW). b) Magnet located in counterclockwise direction $(\mathrm{CCW})$. 


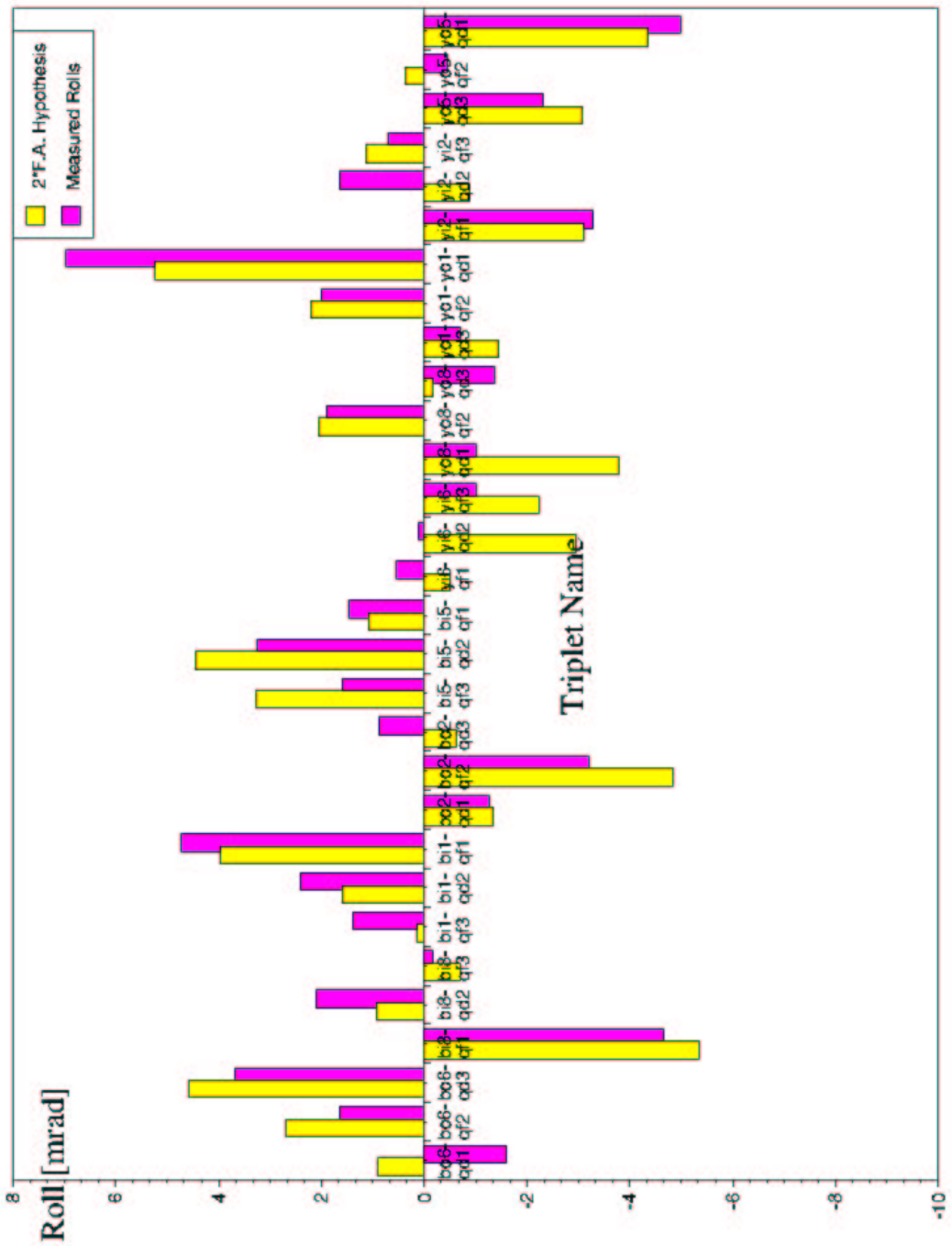

Figure 6.5: The roll angles of some of the IR quadrupoles were measured during the RHIC 2002 shutdown period and compared with the twice the field angles measured before the installation of the magnets. 
Summary of Field Angle Measurements in the Tunnel

\begin{tabular}{|c|c|c|c|c|c|c|c|c|c|c|c|}
\hline Sector & Ring & Q_ID & coldMass & $\begin{array}{l}\text { Meas. } \\
\text { Date }\end{array}$ & Run & $\begin{array}{l}\text { Viewed } \\
\text { from }\end{array}$ & $\begin{array}{c}\text { Quad } \\
\text { Field } \\
\text { Polarity }\end{array}$ & $\begin{array}{c}\text { Uncorrectided } \\
\text { Field Angle } \\
\text { (mrad) }\end{array}$ & $\begin{array}{c}\text { Calibration } \\
\text { Ofrset } \\
\text { (mrad) }\end{array}$ & $\begin{array}{c}\text { Intworal- } \\
\text { Center } \\
\text { Difference } \\
\text { (mrad) }\end{array}$ & $\begin{array}{l}\text { Estimated } \\
\text { Introgral } \\
\text { Fld, Ang. } \\
\text { (mrad) }\end{array}$ \\
\hline 5 & B-1 & CQ1102 & QRI121 & 6772002 & 4 & NLE & -1 & 2.05 & 0.46 & -0.13 & 1.46 \\
\hline 5 & B-1 & CQ2102 & QRK1OB & $6 / 7 / 2002$ & 20 & NLE & 1 & 3.85 & 0.46 & -0.15 & 3.25 \\
\hline 5 & B-I & $\cos 3102$ & QRJ117 & $6 / 7 / 2002$ & 11 & LE & -1 & 2.32 & 0.46 & -0.27 & 1.59 \\
\hline 5 & $Y=0$ & CQ1101 & QRI103 & $6 / 9 / 2002$ & 2 & NLE & -1 & -4.52 & 0.46 & $? ?$ & 4.98 \\
\hline 5 & $Y=0$ & CQ2103. & QRK103 & $6 / 9 / 2002$ & 10 & NLE & 1 & -0.22 & 0.46 & 0.22 & -0.46 \\
\hline 5 & $Y=0$ & cQ3103 & QRJ114 & $6 / 9 / 2002$ & 11 & LE & -1 & -1.58 & 0.46 & -0.26 & -2.30 \\
\hline 6 & $B-0$ & cQ1107 & QRI115 & $6 / 14 / 2002$ & 3 & NLF & -1 & 1.65 & 0.46 & 0.40 & 1.59 \\
\hline 6 & 80 & CQ2124 & QRK112 & $6 / 14 / 2002$ & 19 & NLE & 1 & -1.76 & 0.46 & 0.59 & -1.63 \\
\hline 6 & $B 0$ & CQ3112 & QRJ121 & $6 / 14 / 2002$ & 14 & LE & -1 & -2.64 & 0.46 & -0.59 & -3.69 \\
\hline 6 & $Y-1$ & CQ1115 & QRI123 & $6 / 12 / 2002$ & 3 & NLE & -1 & -0.57 & 0.46 & 0.48 & -0.55 \\
\hline 6 & $Y-1$ & cQ2107 & QRK125 & $6 / 12 / 2002$ & 10 & NLE & 1 & 0.33 & 0.46 & 0.03 & -0.10 \\
\hline 6 & $Y-]$ & $\cos 3113$ & QRJ120 & $6 / 12 / 2002$ & 14 & LE & -1 & 1.92 & 0.46 & -0.46 & 1.00 \\
\hline$B$ & $8-1$ & CQ1121 & QRI109 & $4 / 29 / 2002$ & 3 & NLE & 1 & 5.08 & 0.60 & 0.19 & 4.67 \\
\hline B & B-1 & CQ2110 & QRK122 & $4 / 29 / 2002$ & 9 & NLE & -1 & -2.06 & 0.60 & 0.56 & -2.10 \\
\hline$B$ & B-1 & cQ3105 & QRJ107 & $4 / 29 / 2002$ & 13 & LE & 1 & 0.74 & 0.60 & 0.03 & 0.17 \\
\hline$B$ & $Y=0$ & CQ1120 & QRI10B & $4 / 25 / 2002$ & 3 & NLE & 1 & $1.0 B$ & 0.46 & 0.41 & 1.02 \\
\hline$B$ & $y=0$ & CQ2123 & QRK109 & $4 / 25 / 2002$ & 10 & NLE & -1 & -1.54 & 0.46 & 0.11 & -1.89 \\
\hline B & $Y=0$ & cQ3125 & QRJ126 & $4 / 24 / 2002$ & 13 & LE & 1 & 2.12 & 0.46 & -0.29 & 1.37 \\
\hline
\end{tabular}

Figure 6.6: The field angles were measured in the tunnel during the RHIC 2002 shutdown period by the magnet department at BNL. All the angles were measured from the Q1 no lead end and they are reported in the last column of the table (J. Animesh Courtesy). 


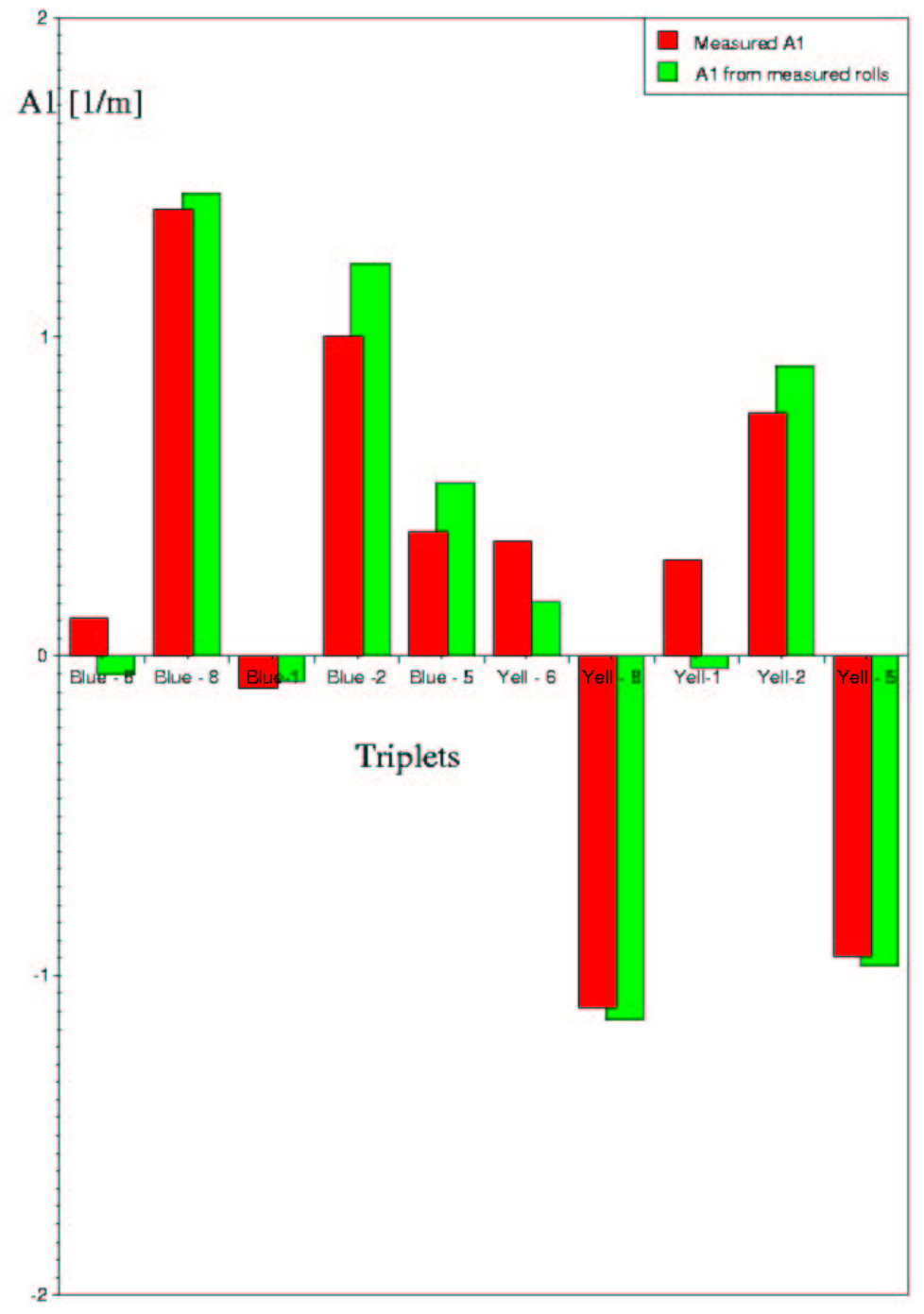

Figure 6.7: The measured $A_{1}$ for all triplets of the Blue ring are compared with the calculated value that uses the measured roll angles. 
strength of the corrector has been added. Except for few triplets the magnetic measurements are in agreement with the orbit measurements and the hypothesis that the triplet magnets have a roll of twice their original field angle. 


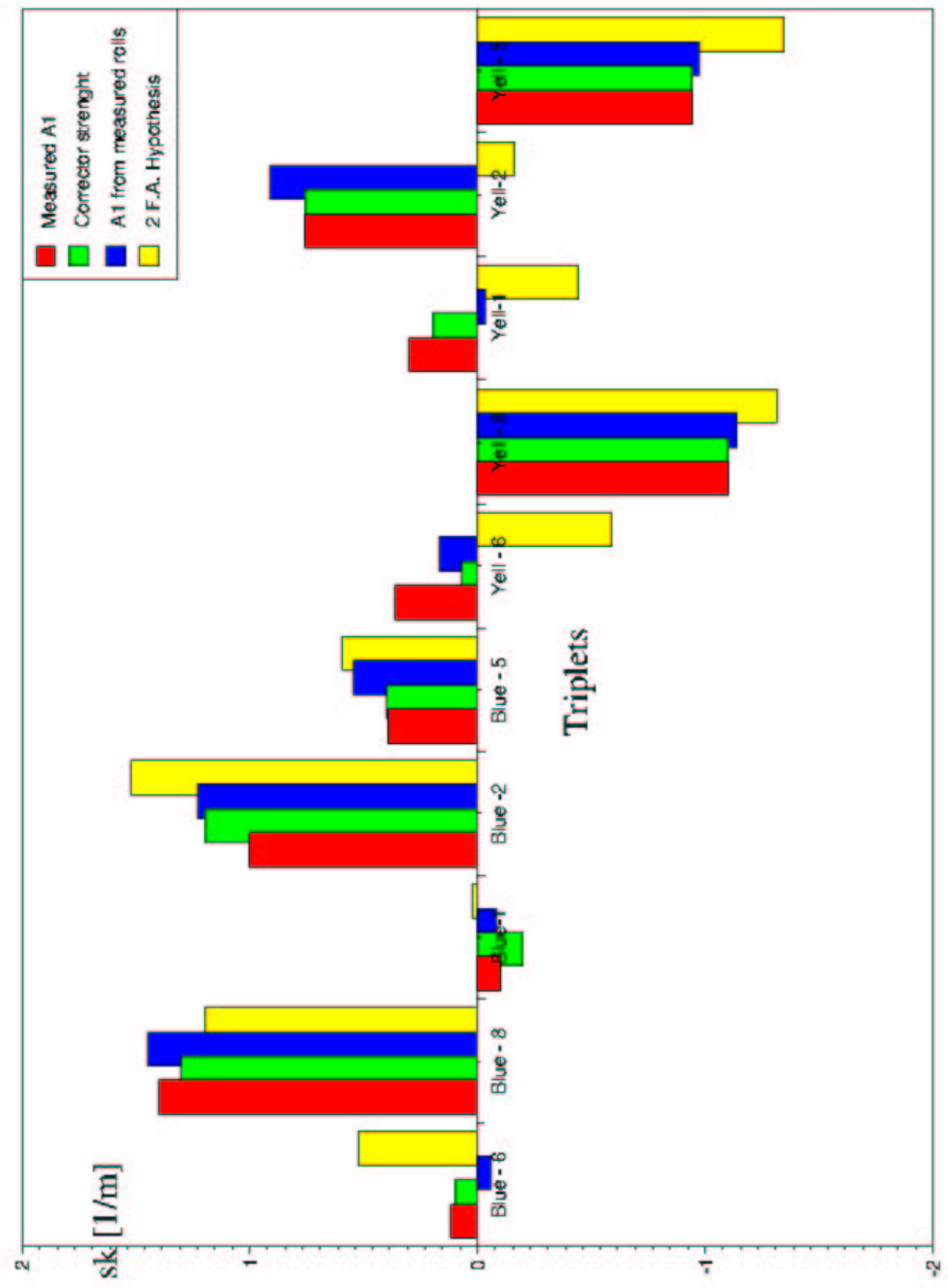

Figure 6.8: The measured values of $A_{1}$ found in Blue and Yellow triplets are compared with the corresponding values calculated from the measured roll angles, the 2 Field angle hypothesis and the value to which the skew quadrupole correctors were set. 


\section{Chapter 7}

\section{Nonlinear Errors}

Section 5.5 describe an experiment aimed to evaluate the nonlinear effects in the determination of the linear coefficients $A_{1}$ and $B_{1}$. Although, the effect of the nonlinearities were found to be significantly smaller that the linear effects on the mentioned experiments, in this chapter we will enhance the technique used in Section 5.5 hoping it would become sensitive enough to measure nonlinear coefficients in the RHIC triplets, specially sextupole components.

\subsection{The Method}

In general, any deviation from the design behavior of a magnet can be expressed by a kick error $\Delta x^{\prime}$ which is equal to:

$$
\Delta x^{\prime}=-\frac{\Delta B_{y} l}{B_{\rho}}
$$

where $\Delta B_{y}$ is the vertical component of the magnetic field error, $l$ is the total length of the magnet and $B_{\rho}$ is the rigidity. There is a similar equation for the 
kick in the vertical plane and it is given by:

$$
\Delta y^{\prime}=\frac{\Delta B_{x} l}{B_{\rho}}
$$

The magnetic error can be either a dipole , a quadrupole or any other multipolar field component.Expressing the magnetic fields in function of their multipolar components we have (see Eq. A.3):

$$
\begin{aligned}
& \Delta x^{\prime}=\left(A_{1} y_{0}-B_{1} x_{0}+2 A_{2} x_{0} y_{0}+B_{2}\left(-x_{0}^{2}+y_{0}^{2}\right)+\ldots\right) \\
& \Delta y^{\prime}=\left(A_{1} x_{0}+B_{1} y_{0}+2 B_{2} x_{0} y_{0}+A_{2}\left(x_{0}^{2}-y_{0}^{2}\right)+\ldots\right)
\end{aligned}
$$

$A_{1}$ corresponds to the skew quadrupole error while $B_{1}$ corresponds to the gradient quadrupole error, $x_{0}$ and $y_{0}$ are the horizontal and vertical position of the beam at some position $s_{0}$ inside the magnetic structure under consideration and $B_{2}$ and $A_{2}$ are the normal and skew sextupole components.

Expansion 7.3 is no longer valid when more than one magnet is involved in the magnetic kick. Equivalent coefficients will arise that depend on the individual coefficients of the magnets that build the whole magnetic structure for which the equivalent magnetic want to be determined and therefore Eq. 7.3 will change (see Eq. B.33). If the magnetic structures are RHIC triplets, it is shown in Appendix B (Eq. B.33) that the equivalent magnetic kick is given by:

$$
\Delta x_{e q}^{\prime}=A_{1}^{e q} y_{0}-B_{1}^{x} x_{0}+2 A_{2}^{x} x_{0} y_{0}-B_{2}^{x a} x_{0}^{2}+B_{2}^{x b} y_{0}^{2}+\ldots
$$




$$
\Delta y_{e q}^{\prime}=A_{1}^{e q} x_{0}+B_{1}^{y} y_{0}+2 B_{2}^{y} x_{0} y_{0}+A_{2}^{y a} x_{0}^{2}-A_{2}^{y b} y_{0}^{2}+\ldots
$$

In this case the coefficients and $s_{0}$ have a slightly different meaning than the ones in Eq. 7.3. Each of the coefficients of Eq. 7.4 represents the multipole that will produce the same effect as the corresponding 3 multipoles in the triplet under study when located at the position $s_{0}$.

As was seen in the previous chapter $\Delta x_{e q}^{\prime}$ and $\Delta y_{e q}^{\prime}$ can be determined experimentally from difference orbit analysis and the beam positions (horizontal and vertical) at $s_{0}$ can be easily inferred from the closest BPMs to $s_{0}$. One possible experiment to determine the multipole coefficients is to excite several betatron oscillations all of them with different amplitudes. That will provide

a set of points $\Delta x_{e q}^{\prime}, \Delta y_{e q}^{\prime}, x_{0}$ and $y_{0}$ (one point for every different amplitude) which can be used to evaluate the coefficients in Eq. 7.4.

These coefficients are directly related with the strengths that should be put in the correctors to locally correct for the error as was shown for the skew quadrupole errors in Chapter 4.

\subsection{Experiment to Find non Linear Errors}

This experiment is essentially the same experiment presented in Section 5.5 except that 10 different corrector strengths are used instead of four and only two dipole corrector are used: bo6-th14 and bo6-tv17. 


\subsubsection{Analysis}

Analysis for individual triplets doesn't seem to be possible from the difference orbits obtained for this experiment since the interaction region BPMs didn't register any measurement. However it is possible to find equivalent multipole errors for the whole interaction region. The dipole correctors used has an optimal phase advance to do the analysis at interaction region 8 .

As it has been discussed earlier the linear and nonlinear errors give a kick to the beam $\Delta x^{\prime}$ in the horizontal and $\Delta y^{\prime}$ in the vertical plane that can be evaluated at the IR of interest from the difference orbits. Graphs of $\Delta x^{\prime}$ vs $x$ or $y$ and $\Delta y^{\prime}$ vs $x$ or $y$ can then be drawn exactly as in the linear experiments.

Fig. 7.1 shows the graphs obtained from the horizontal dipole corrector difference orbits while Fig. 7.2 show the corresponding graphs obtained from the vertical dipole corrector difference orbits. The graphs are mainly linear but it is possible to note a slight curvature specially in Fig. 7.1 which might be an indication of the non linear components present at the interaction region.

Fitting the graphs produced by the horizontal dipole corrector to the polynomial equation:

$$
\begin{aligned}
& \Delta x^{\prime}=b^{x a}+c_{1}^{x a} x_{0}+c_{2}^{x a} x_{0}{ }^{2}+\ldots \\
& \Delta y^{\prime}=b^{y a}+c_{1}^{y a} x_{0}+c_{2}^{y a} x_{0}{ }^{2}+\ldots
\end{aligned}
$$




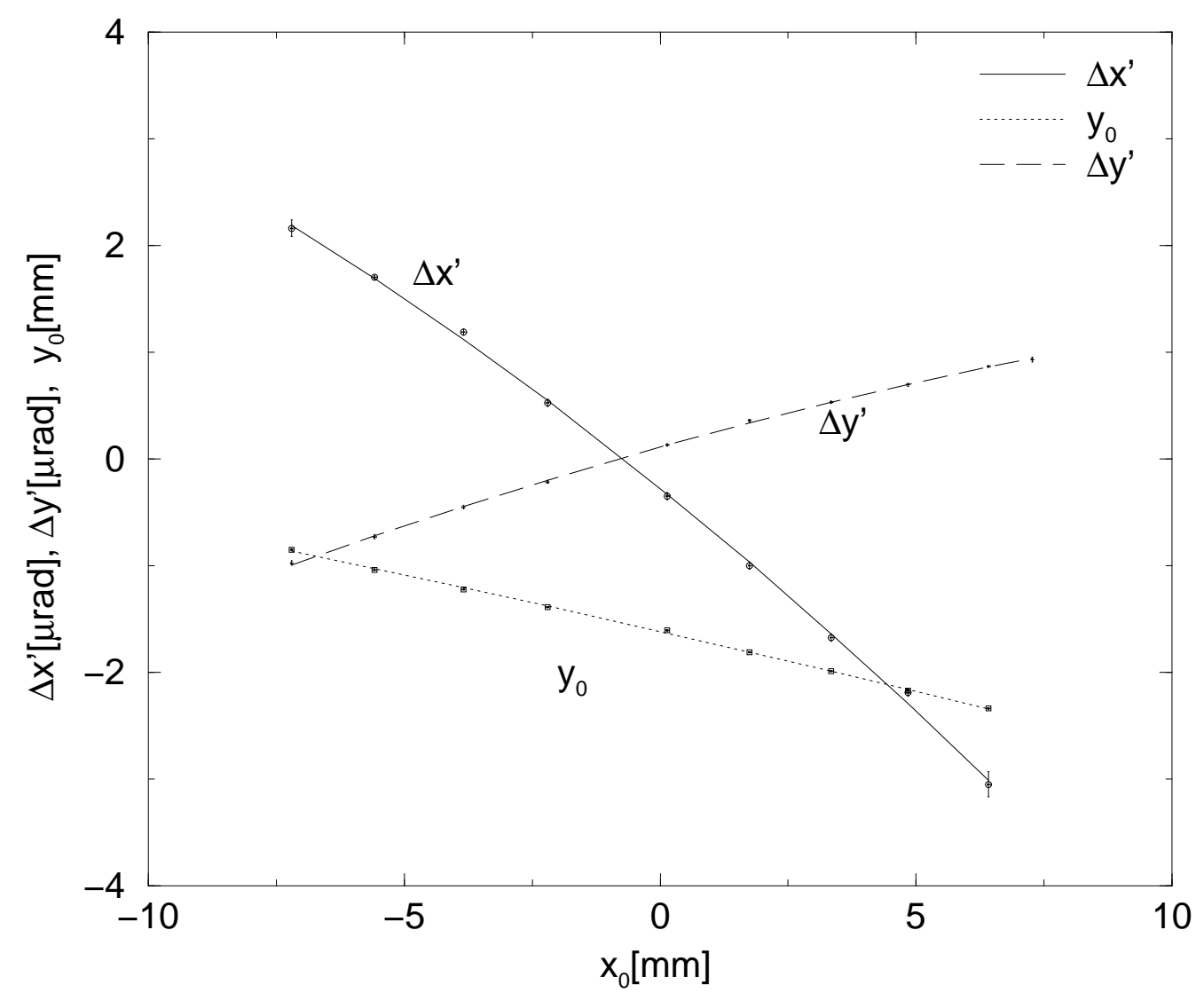

Figure 7.1: Graphs of magnetic kick vs beam position extracted from BPM measurements obtained by changing the strength of the horizontal dipole corrector bo6-th14. Even though the linear errors dominated these curves, nonlinear behavior are also present and it is possible to determine such nonlinearities from polynomial fits. 


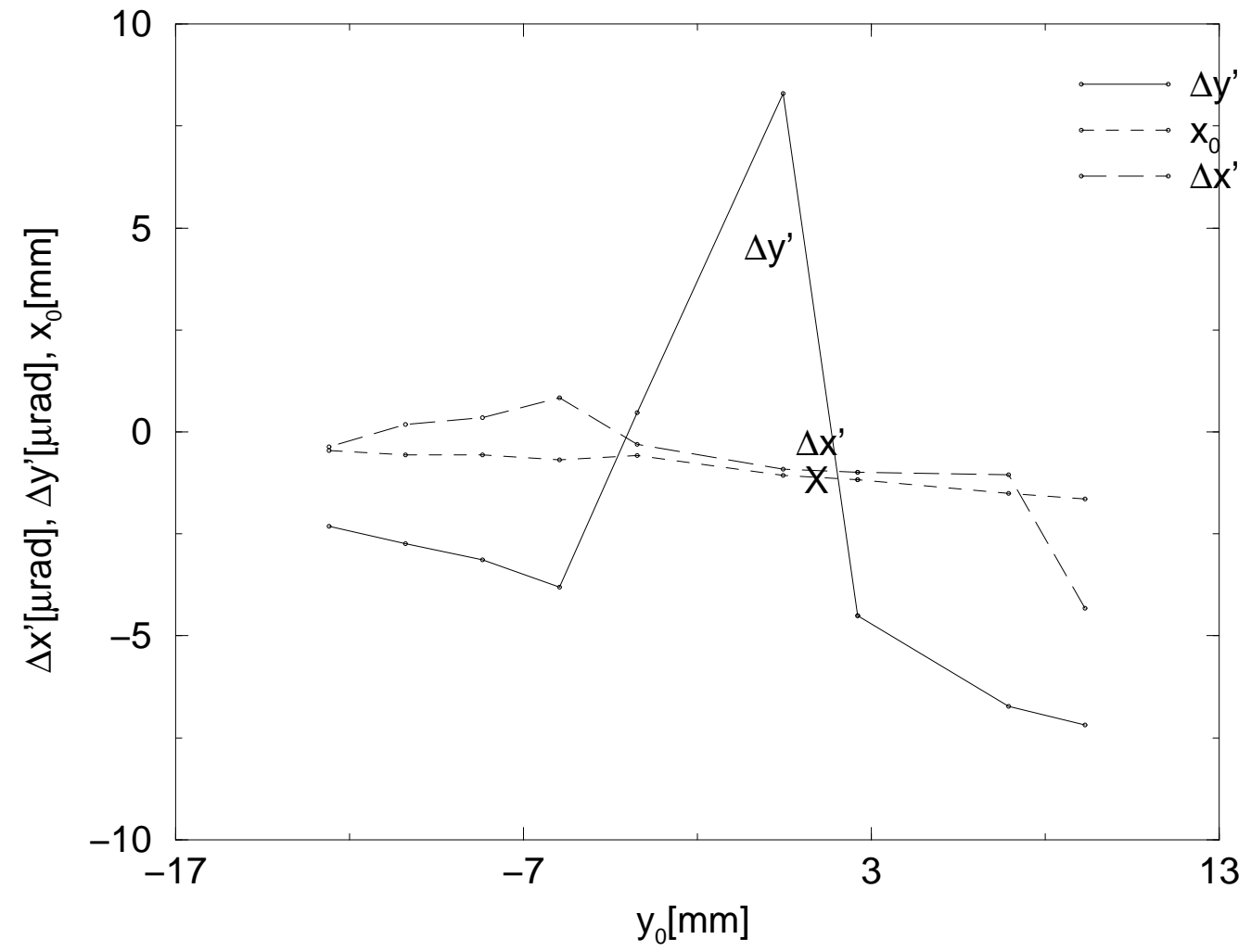

Figure 7.2: Graphs of magnetic kick vs beam position extracted from BPM measurements obtained by changing the strength of the vertical dipole corrector bo6-tv17. Some of the points in these graph don't follow the expected linear trend of the curves in some cases due to poor fittings of the original orbits. 
and fitting the graphs produced by the vertical dipole corrector to the polynomial equation:

$$
\begin{aligned}
& \Delta x^{\prime}=b^{x b}+c_{1}^{x b} x_{0}+c_{2}^{x b} x_{0}^{2}+\ldots \\
& \Delta y^{\prime}=b^{y b}+c_{1}^{y b} x_{0}+c_{2}^{y b} x_{0}^{2}+\ldots
\end{aligned}
$$

it is possible to find the coefficients $c_{1}^{x b}, c_{2}^{x b}, c_{1}^{y b}$ and $c_{2}^{y b}$ which can be directly related to the equivalent multipolar coefficients. Eq. 5.25 shows the relation between the multipolar coefficients and the equivalent linear errors. The relation between the quadratic coefficients and the equivalent sextupole errors will be shown in the next paragraph.

Assuming that the contribution of the octupole components are very small it is possible to rewrite Eq. 7.4 as:

$$
\begin{aligned}
& \Delta x^{\prime}=-B_{1}^{x} x-B_{2}^{x a} x^{2}+A_{1} y+2 A_{2}^{x} x y+B_{2}^{y} y^{2} \\
& \Delta y^{\prime}=A_{1} x+A_{2}^{x} x^{2}+B_{1}^{y} y+2 B_{2}^{y} x y-A_{2}^{y a} y^{2}
\end{aligned}
$$

Using the relations between $\mathrm{y}$ and $\mathrm{x}, y=m_{a} x+b_{a}$, and Eq. 7.5 in Eq. 7.7, it is found that:

$$
c_{2}^{x a}=B_{2}^{x a}+2 A_{2}^{x} m_{a}+B_{2}^{y} m_{a}{ }^{2}
$$




$$
c_{2}^{y a}=A_{2}^{x}+2 B_{2}^{y} m_{a}-A_{2}^{y a} m_{a}{ }^{2}
$$

Similarly for the coefficients associated with the vertical dipole corrector orbits we have:

$$
\begin{aligned}
c_{2}^{x b} & =B_{2}^{y}+2 A_{2}^{x} m_{b}-B_{2}^{x a} m_{b}{ }^{2} \\
c_{2}^{y b} & =-A_{2}^{y a}+2 B_{2}^{y} m_{b}+A_{2}^{x} m_{b}{ }^{2}
\end{aligned}
$$

where $m_{b}$ comes from the relation $x=m_{b} y+b_{b}$. Eq. 7.8 and 7.9 define a four by four linear equation system with solution:

$$
\begin{aligned}
A_{2}^{y a} & =-\left(\frac{-c_{2}^{y b}+2 c_{2}^{x b} m_{b}+3 c_{2}^{y b} m_{a} m_{b}-3 c_{2}^{y a} m_{b}{ }^{2}-2 c_{2}^{x a} m_{b}{ }^{3}+c_{2}^{y a} m_{a} m_{b}{ }^{3}}{\left(-1+m_{a} m_{b}\right)^{3}}\right) \\
A_{2}^{x} & =-\left(\frac{c_{2}^{y a}-2 c_{2}^{x b} m_{a}-c_{2}^{y b} m_{a}{ }^{2}+c_{2}^{y a} m_{a} m_{b}-c_{2}^{y b} m_{a}{ }^{3} m_{b}+2 c_{2}^{x a} m_{a} m_{b}{ }^{2}}{\left(-1+m_{a} m_{b}\right)^{3}}\right) \\
B_{2}^{y} & =-\left(\frac{c_{2}^{x b}-2 c_{2}^{y a} m_{b}+c_{2}^{x b} m_{a} m_{b}+2 c_{2}^{y b} m_{a}{ }^{2} m_{b}-c_{2}^{x a} m_{b}{ }^{2}-c_{2}^{x a} m_{a} m_{b}{ }^{3}}{\left(-1+m_{a} m_{b}\right)^{3}}\right) \\
B_{2}^{x a} & =-\left(\frac{-c_{2}^{x a}+2 c_{2}^{y a} m_{a}-3 c_{2}^{x b} m_{a}{ }^{2}-2 c_{2}^{y b} m_{a}{ }^{3}+3 c_{2}^{x a} m_{a} m_{b}+c_{2}^{x b} m_{a}{ }^{3} m_{b}}{\left(-1+m_{a} m_{b}\right)^{3}}\right)
\end{aligned}
$$

\subsubsection{Results}

Before fitting the the curves in Fig. 7.1 and Fig. 7.2 it is neccesary to make some observations about these figures. 
The curve that relates $\Delta y^{\prime}$ vs $y_{0}$ in Fig. 7.2 has 2 points that are well beyond the general trend of the other points of the same curve. Such 2 points correspond to the orbits that were taken when the strength of the dipole corrector bo6-tv17 was set to +0.02 radians and -0.02 radians. While the orbit taken with dipole strength equal to -0.002 radians doesn't fit properly to the model, the orbit taken with dipole strength equal to +0.002 radians agrees pretty well with the model. The only possibility to explain the problem in the +0.02 setting is the presence of a systematic error related with the baselines.

Discarding the 2 anomalous points in Fig. 7.2, the fit leads to the coefficients that are listed in Table 7.1

\begin{tabular}{|l|r|}
\hline$c_{1}^{x a}$ & $-0.399 \pm 0.006$ \\
$c_{1}^{x b}$ & $-0.185 \pm 0.002$ \\
$c_{1}^{y a}$ & $0.137 \pm 0.003$ \\
$c_{1}^{y b}$ & $-0.151 \pm 0.002$ \\
$m_{a}$ & $-0.104 \pm 0.002$ \\
$m_{b}$ & $-0.070 \pm 0.003$ \\
$c_{2}^{x a}$ & $-0.0034 \pm 0.0025$ \\
$c_{2}^{x b}$ & $0.0062 \pm 0.0011$ \\
$c_{2}^{y a}$ & $-0.0026 \pm 0.0010$ \\
$c_{2}^{y b}$ & $-0.0130 \pm 0.0003$ \\
\hline
\end{tabular}

Table 7.1: Linear and Quadratic Coefficients after fitting data to Eq. 7.5 and Eq. 7.6. Linear coefficients are given in $\mu \mathrm{rad} / \mathrm{mm}$ while the quadratic coefficients are given in $\mu \mathrm{rad} / \mathrm{mm}^{2}$.

In order to find all coefficients with subscript $a$ the graphs of Deltas were fit to a quadratic polynomial, then a third order polynomial and so on. $\chi^{2}$ was evaluated in each case and the polynomial which give the $\chi^{2}$ closer to one 
was chosen. The fitting procedure was done with a special routine of EXCELL (SOLVED) which easily allow to find the coefficients of the polynomial that minimize the $\chi^{2}$.

This procedure looks to minimize as much as possible the systematic error produced when significant multipole components are ignored in the fitting or when more multipoles than needed are consider in the fitting.

The coefficients with subscript $b$ didn't go trough the previous procedure. Only one fit to a cubic polynomial was done for those coefficients since that was the most common optimal polynomial in the analysis to obtain the coefficients with subscript $a$.

The errors shown on the right hand side are propagated errors and no direct statistical error measurements like in the previous tables.

The calculation of the propagated errors were done according to reference [32]. If a function of the form:

$$
y\left(x_{i}\right)=\sum_{k=1}^{m} a_{k} f_{k}\left(x_{i}\right)
$$

is linear in the parameters $a_{k}$ and the functions $f_{k}\left(x_{i}\right)$ are the monomials $f_{1}(x)=1, f_{2}(x)=x, f_{3}(x)=x^{2}$ and so on, then it is possible to define a matrix:

$$
\alpha_{l k} \equiv \sum\left[\frac{1}{\sigma_{i}^{2}} f_{l}\left(x_{i}\right) f_{k}\left(x_{i}\right)\right]
$$

with an inverse $\alpha^{-\mathbf{1}}$ that correspond to the error matrix $\sigma^{2}$. The diagonal terms of this matrix are the variances associated with the linear coefficients, quadratic coefficients, cubic coefficients, etc. 
In order to apply Eq. 7.12 it is necessary to know the uncertainty associated with each $y_{i}$ point $\sigma_{i}$. This uncertainty can be calculated from the uncertainty associated with each $x_{i}$ and propagating this uncertainty through Eq. 7.7. The uncertainty associated with each $x_{i}$ was calculated from the variations of this variable when different baselines taken during the experiment are used to produce the difference orbits.

A perl script (GET_COEFF_ERROR) was created to calculate the propagated uncertainty $\sigma_{i}$ and the $\alpha$ matrix from a set of 5 graphs of delta vs positions. Such graphs differ only in the baseline used in each case.

Once the $\alpha$ matrix is found, it is fed into a Mathematica notebook to invert the matrix and find the error matrix for the coefficients.

Linear regressions of the curves $x_{0}$ vs $y_{0}$ and $y_{0}$ vs $x_{0}$ performed in the application software XMGR also give the errors associated with $m_{a}$ and $m_{b}$.

The next step is the calculation of the equivalent multipole coefficients with Eq. 7.10 and their propagated errors with the general formula:

$$
\sigma_{z}^{2}=\sum\left[\sigma_{i}^{2}\left(\frac{\partial z}{\partial y_{i}}\right)^{2}\right]
$$

Those calculations has been done in Mathematica notebooks (sext coeff2.nb and linear3coeff2.nb) with results that can be seen on Table 7.2. Linear errors have small uncertainties associated while nonlinear errors have associated errors of the same order of magnitude of the error itself. Further studies are required to determine what variables causes the biggest uncertainties in the non linear coefficients. By now, it is possible to guess that if the number of 


\begin{tabular}{|c|r|}
\hline$A_{1}^{e q}$ & $0.122 \pm 0.003$ \\
$B_{1}^{x}$ & $0.386 \pm 0.001$ \\
$B_{1}^{y}$ & $-0.142 \pm 0.002$ \\
$A_{2}^{y a}$ & $0.0121 \pm 0.0003$ \\
$A_{2}^{x}$ & $-0.0012 \pm 0.0011$ \\
$B_{2}^{y}$ & $0.0061 \pm 0.0011$ \\
$B_{2}^{x a}$ & $0.0037 \pm 0.0025$ \\
\hline
\end{tabular}

Table 7.2: Equivalent Multipole Coefficients.The units are $\frac{\mu \mathrm{rad}}{\mathrm{mm}}$ or $10^{-3} \frac{1}{\mathrm{~m}}$ for the linear coefficients and $\frac{\mu \mathrm{rad}}{\mathrm{mm}^{2}}$ or $\frac{1}{\mathrm{~m}^{2}}$.

different settings of the dipole correctors are increased the uncertainty will go down. Also a better behavior of the vertical plane orbits will improve the situation. Measurements in the triplets and no in the whole interaction region will probably reduce the uncertainty too. Eq. 7.12 shows clearly that an increase of the amplitude of the oscillations will increase alpha making the errors smaller. Original experiments were done by exciting betatron oscillations in one plane and looking to the induced oscillations in the opposite plane. Next experiment will be done with betatron oscillations excited in both planes which will decrease the uncertainty according to the analysis done previously. At this time (August 2002) there is no data available to do direct comparisons with the values found for the sextupole coefficients in blue ring in the particular IR that was studied. However, measurements were done before the end of the RHIC run 2002 in the yellow ring with an alternate technique. This measurements have at least the same order of magnitude (see the RHIC elog book Jan 32002 and [33]) as the values found here for the sextupole coefficients. 


\section{Chapter 8}

\section{Calibration Experiments}

RHIC has packages of correctors that range from dipole correctors to dodecapole correctors that have been calibrated before installation in the tunnel. The correctors are activated through a special software (Ramp Editor Manager) that allows to set any corrector to a desired corrector strength.

Experiments in this chapter are aimed to find the relation between the ramp editor manager strengths to which some quadrupole and sextupole correctors are set and the corresponding values obtained with the action and phase analysis of the resultant orbits.

\subsection{Calibration Experiments for Skew Quadrupole Correctors}

This experiment consist of a set of first turn difference trajectories taken with different settings in a particular skew quadrupole corrector. Action and phase analysis and Eq. 5.17 then allow to measure the particular strength to 
which the skew quadrupole was set.

Fig. 8.1 shows the relation between the set value (number written in the RHIC ramp editor window) on the right skew quadrupole corrector at IR8 and the corresponding measured skew strength obtained from the action and phase analysis.

In order to find the $A_{1}$ from the orbit measurements, it is neccesary to use the horizontal and the vertical plane of the orbit. The fit of the horizontal orbit to the model is good but not in the vertical plane. Nonetheless, the overall trend of the calibration curve shown in Fig. 8.1 is as expected. Notice also that the slope is negative, which is in agreement with the results obtained in Appendix F, Eq. F.5.

\subsection{Calibrating Gradient Error Measurements}

It was shown in Chapter 5 that precise measurements of the equivalent gradient errors $\left(B_{1}^{x}\right.$ and $\left.B_{1}^{y}\right)$ at RHIC IRs can be done with the action and phase method. The accuracy of this technique can also be evaluated with the help of the action and phase method by inserting known values of gradient errors. Those gradient errors were inserted by varying the strength of the quadrupole bo7-qd1 by known quantities.

For each strength variation of bo7-qd1, a set of orbits are taken by varying the strengths of a horizontal dipole corrector and the same procedure is repeated for a vertical dipole corrector. The equivalent magnetic kick $\Delta x^{\prime}$ and 


\section{Skew Quadrupole Calibration}

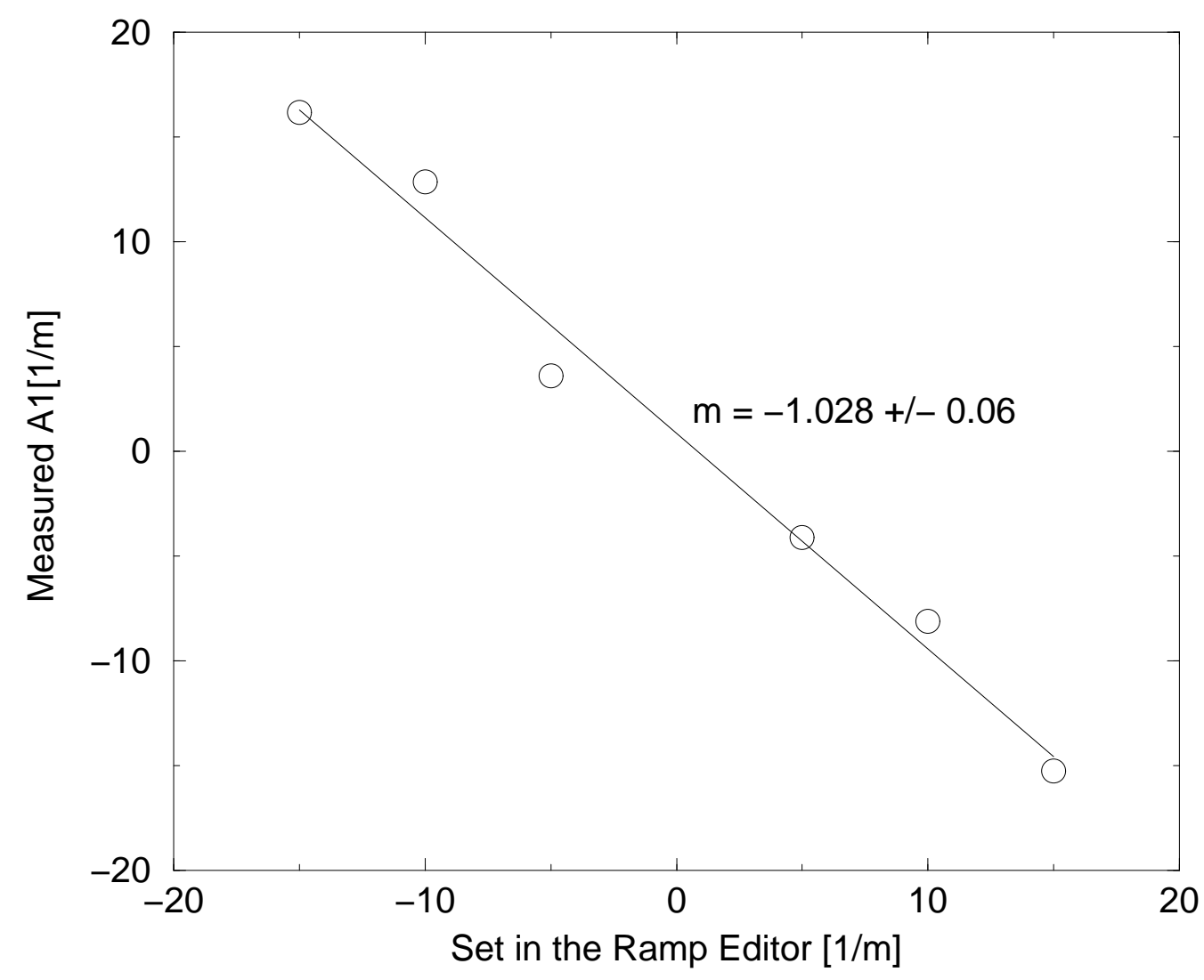

Figure 8.1: Calibration curve for the right skew quadrupole corrector (bi8-qs3 or SQ08C2B) at IR8. 


\section{Calibration Curve for bo7-qd1}

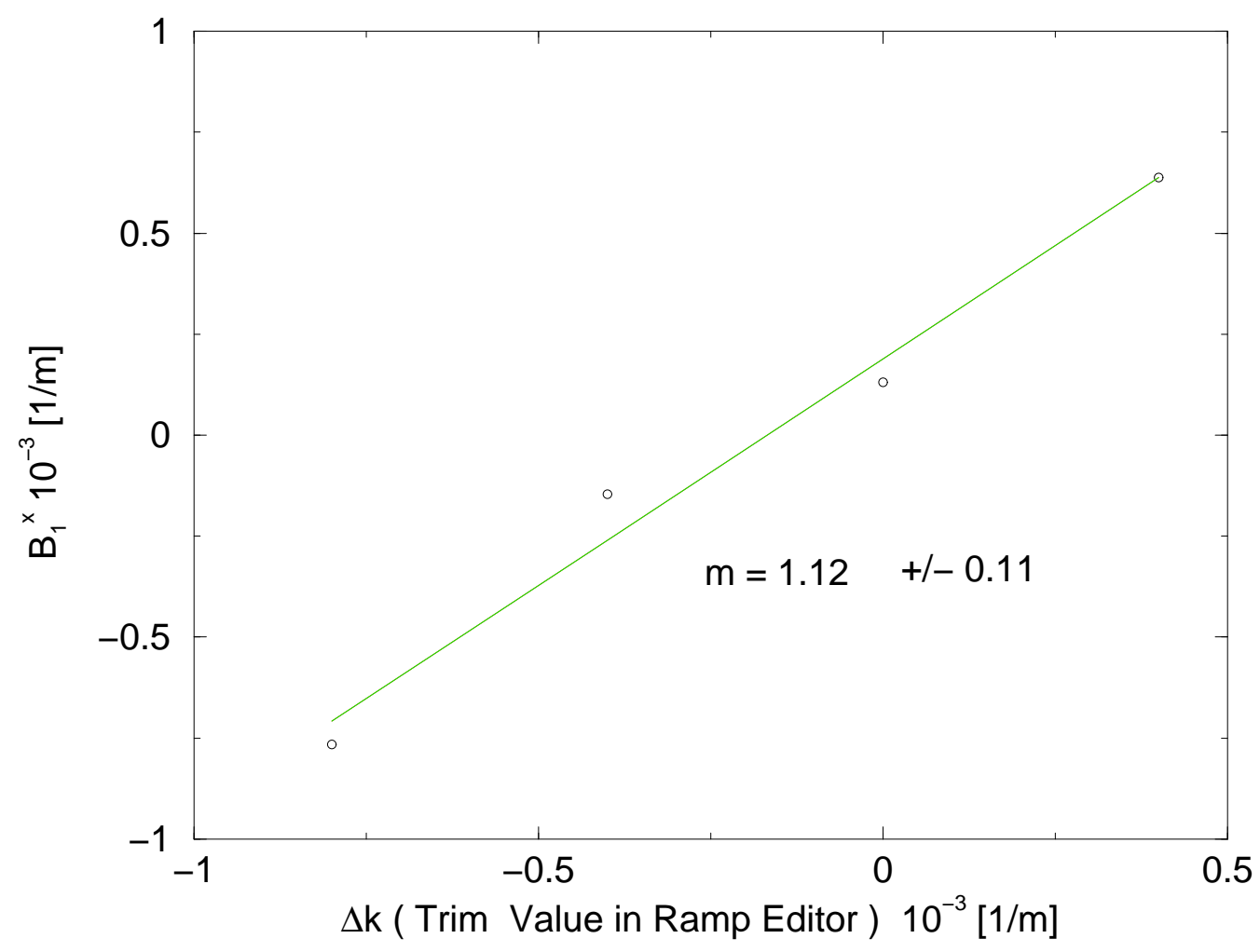

Figure 8.2: The strength of bo7-qd1 is varied in the RHIC ramp editor manager while the equivalent gradient error $B_{1}^{x}$ is measured.

$\Delta y^{\prime}$ at the relevant IR for each orbit are then evaluated by using Eq. 5.16 leading to graphs like the one shown in Fig. 5.2. The equivalent gradient errors can be evaluated from the mentioned figures using Eq. 5.25. Fig. 8.2 and Fig. 8.3 show the results of the previous analysis.

Since the $\Delta x^{\prime}, \Delta y^{\prime}, y$ and $x$ are measured at exactly the location where the bo7-qd1 is located, the equivalent gradient error of a RHIC triplet expressed 


\section{Calibration Curve for bo7-qd1}

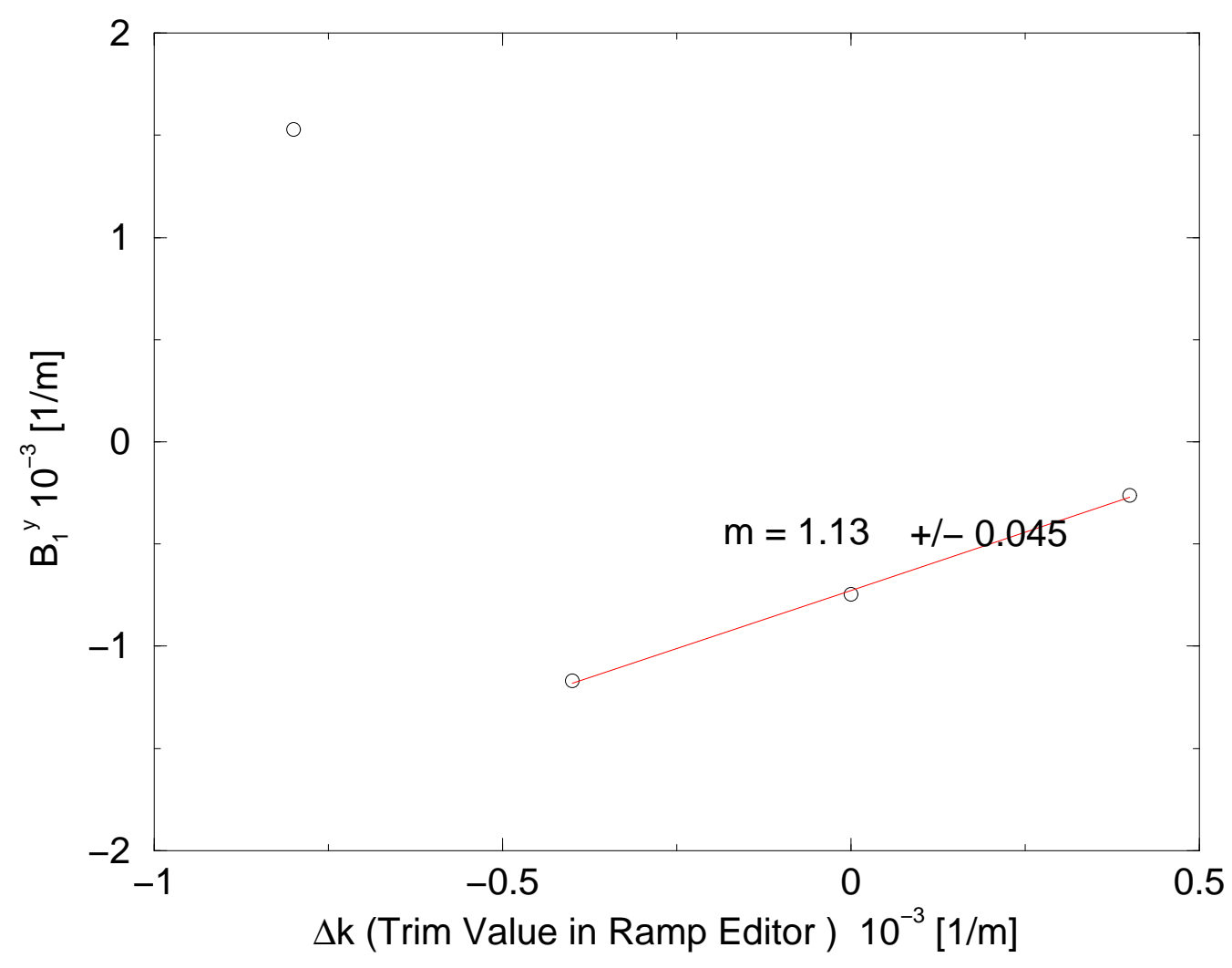

Figure 8.3: Same as Fig. 8.2 but now measuring $B_{1}^{y}$. The precision of this experiment is better when compared to the previous case revealing the existence of a systematic error of about 0.1 in the slope of the curve. 
in function of the individual gradient errors at each quadrupole of the triplet is reduced to:

$$
B_{1}^{z e}=B_{1}^{a}+\frac{\beta_{z b} B_{1}^{b}+\beta_{z c} B_{1}^{c}}{\beta_{z a}}
$$

where Eq. 8.1 follows from Eq. B.16 when $\beta_{z e}$ (with ze representing either $x e$ or $y e)$ is made equal to $\beta_{z a}$. The relation between the measured value $B_{1}^{z e}$ and the value used in bo7-qd1 is then expected to be 1:1 according to Eq. 8.1. Fig. 8.2 and Fig. 8.3 are not only linear relations but also reproduce the correct slope with a precision that can be as low as $4 \%$ and a accuracy below $10 \%$. It is important to emphasizes that these numbers are related to the gradient error not to the total strength of the particular quadrupole under study. The total strength can be found by adding the gradient error to the design strength or to the strength of the respective quadrupole used in the model in which the measurements will be based. The error in the determination of the total strength of the quadrupole can be as small as $0.06 \%$ according to these results.

\subsection{Sextupole Corrector Calibration Experi- ment}

During the RHIC 2003 deuteron-gold run additional calibration experiments with sextupoles were performed. The experiment is basically to set a sextupole corrector to some known strength and then take a series of orbits with different strengths of a particular dipole corrector. The experiment is then repeated for other 3 different sextupole corrector strengths. 
Graphs of $\Delta x^{\prime}$ and/or $\Delta y^{\prime}$ vs $x$ can be obtained for each different sextupole strength used (see Fig. 8.4) and a fit of the curves to Eq. B.33 can be used to evaluate the sextupole coefficients.

The normal sextupole coefficients $\left(B_{2}^{x a}\right.$ and $\left.B_{2}^{y}\right)$ obtained from the fits to the different set of measurements are function of the sextupole strength applied to the sextupole corrector used in the experiment (bo7-sx3). If the magnetic kicks and the beam positions in the previous graphs are measured at the location of the sextupole corrector, the functions that relate $B_{2}^{x a}$ and $B_{2}^{y}$ with the sextupole corrector strength $B_{2}^{\text {corr }}$ are:

$$
\begin{aligned}
& B_{2}^{x a}=B_{2}^{\text {corr }}+B_{2}^{\text {tripx }} \\
& B_{2}^{y}=B_{2}^{\text {corr }}+B_{2}^{\text {tripy }}
\end{aligned}
$$

where $B_{2}^{\text {tripx }}$ and $B_{2}^{\text {tripy }}$ are the normal sextupole coefficients produced by the combination of sextupole errors in all 3 quadrupoles of the triplet. Therefore, measuring $B_{2}^{x a}$ or $B_{2}^{y}$ is like measuring $B_{2}^{\text {corr }}$ plus and offset given by the original sextupole errors present at the quadrupoles of the triplet.

The calibration curve (measured value vs set values) obtained from the different series of measurements can be seen in Fig. 8.5. The uncertainties associated with each measurement are propagated errors derived from the estimated errors of the graphs of magnetic kick versus position from which the calibration curve was obtained. The calibration curve follows the expected 
trend but the propagated errors seem to be very small considering how much the points are scattered around the calibration curve. There are evidence that errors associated with the magnetic kicks from which the sextupole were extracted were underestimated. Indeed the quadratic fits done to the curves of magnetic kick versus the beam position (see Fig. 8.4) give values for $\chi^{2}$ equal to 2.3, an indication of too small uncertainties. The uncertainties associated with RHIC 2001 proton experiments magnetic kicks were 4 times smaller than the ones found in the RHIC 2003 dAu experiments. This might be due to some temporary condition of the machine but also might be related with the particle used. If this is the case, then it will be convenient to repeat this experiment with protons. Another factor that will reduce the errors is increasing the number of points used to determine each sextupole strength. Due to the time limitations only 4 points per sextupole strength were used in the RHIC 2003 dAu experiment. Increasing the amplitude of the betatron oscillation will definitively help to resolve the strength with better precision but the feasibility of increasing the amplitude beyond the maximum amplitude used of about 10 $\mathrm{mm}$ must be carefully examined. 


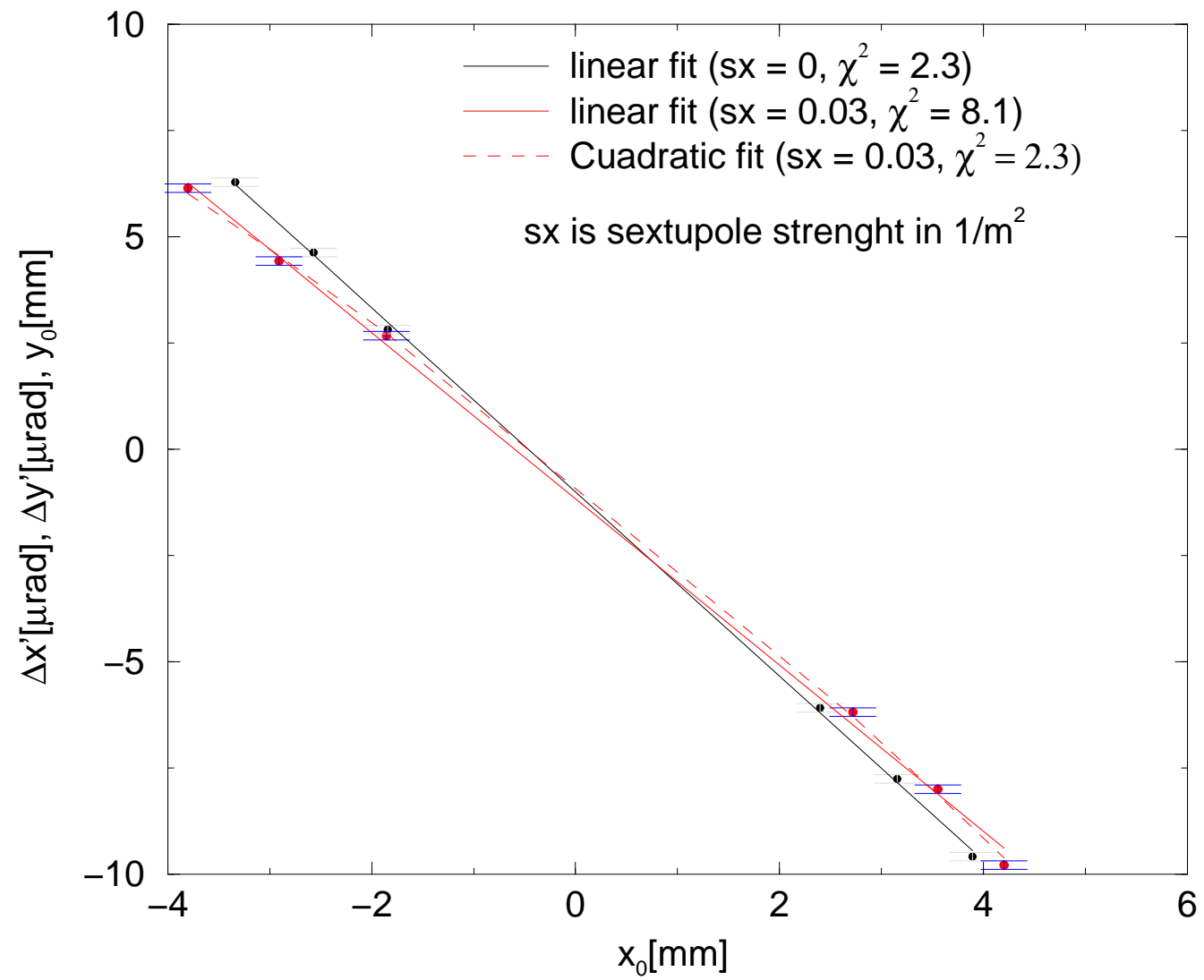

Figure 8.4: Measurements of magnetic kick vs beam position at the location of the sextupole corrector used in the sextupole calibration experiment. Evaluation of $\chi^{2}$ for different sextupole strengths and polynomial fits makes evident the effect of the sextupoles in the orbit measurements. 


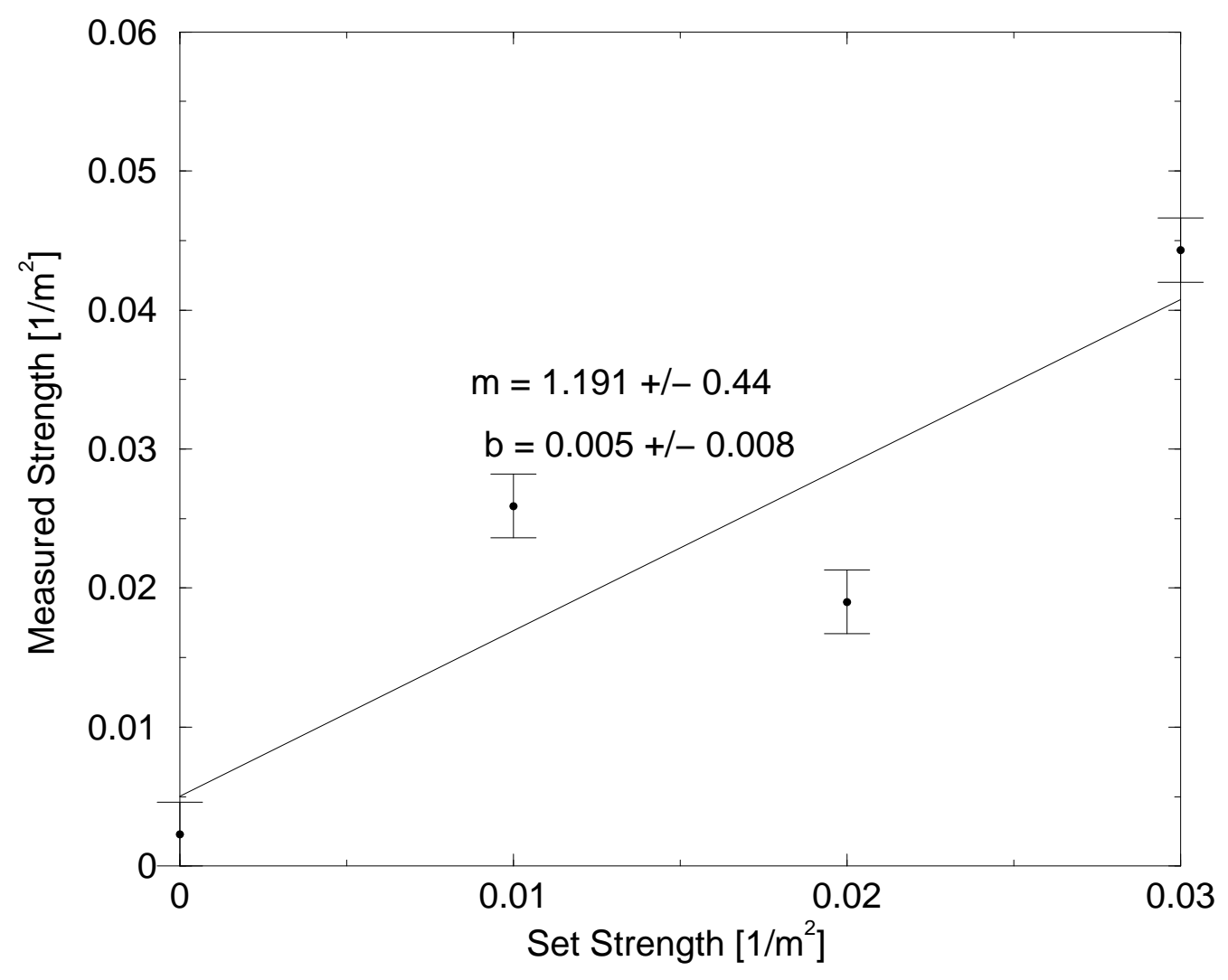

Figure 8.5: Sextupole calibration curve obtained with difference orbits collected during the RHIC 2003 dAu run. 


\section{Chapter 9}

\section{Software for Analysis of First Turn Orbits and Closed Orbits}

Most of the orbit analysis presented in this thesis requires extensive computations that can be efficiently performed by well designed programs. This chapter shows a complete description of the software that was developed in order to simplify the analysis of the experiments. The software has been design such that it might be possible in the future to do on-line measurements. All the software presented in this section is saved in the BNL public directory /rap/lattice_tools/ActionPhase/ unless otherwise indicated.

\subsection{Preprocessing the Orbit Data}

Orbit data in RHIC is stored in files *.sdds, which are convenient to have in ASCII format in order to easily process the orbit. The conversion to ASCII is done with a special application called SDDSCONVERT available in the SUN machines in the directory /usr/local/bin/. 
Difference orbits are the most common type of orbit used in this thesis. The RHIC Orbit Display Program has a very useful menu function that allow to do difference between two orbits in a windows environment. This function is enough when few orbits are being analyzed at the same time, but when it is neccesary to do analysis with a large number of orbits this process can become very slow. Line commands or scripts are preferred in those cases and several of them has been written in $\mathrm{C}++$ code and PERL.

BSUBSTRACT.C is a $\mathrm{C}++$ program that take 2 multi-turn orbits in ASCII format, jump to the fifth orbit, which is the actual first injection turn, and performed the difference between the two orbit measurements of each BPM. The results are saved in the same ASCII format as the original orbits are saved. Besides the first injection turn, the measurements of arc 6-8 corresponding to the second turn are also processed which are necessary to evaluate errors at IR6. There is also a version in which arc 6-8 orbit data is not processed (SUBS_IMPR) and different to BSUBSTRACT.C is a PERL script.

Closed orbits, different to multi-turn orbit, are stored in one turn files that requires a slight different program (BSUBS.CLOSE) to do the difference orbit.

It was not always possible to do the difference orbits with the programs described. There is a peculiar behavior with some of the orbits that I will described in the next paragraphs.

A typical line of an orbit file is: 
$s x x c c f s y y c c f$

where $s x$ is the position of a horizontal BPM, $x$ is the corresponding BPM reading, sy is the position of a vertical BPM, $y$ is the corresponding BPM reading and $c$ and $f$ are control characters

The programs described earlier rely in the fact the that two orbit files used to do the difference orbit are aligned when comparing column $s x$ and $s y$. This means that if a file has in one of its lines $s x=3000$ and $s y=3100$ the other file should have the same values in the same line. This is a fact that even the Rhic orbit display program uses to do the difference orbits.

Sometimes orbits that don't satisfy the above criteria are found and the programs to do the difference orbits fail.

The program SUBS_TRICKY (only closed orbits) was created to solve the mentioned problem. In this program, both orbits are read completely before processing them. Relevant values are stored in matrices that later are read to do the corresponding operations and write the final difference orbit file.

Besides the orbit data, the software developed needs a Twiss file with the designed lattice functions. This lattice file has a format very similar to the input lattice file of the Rhic Orbit Display Program. A simple $\mathrm{C}++$ program called CONVERTTWISS is used to convert the input lattice file of the Rhic Orbit Display Program into the input lattice file of the software presented here. In order to run this program, it is neccesary to give the name of the lattice input file of the Rhic Orbit and the name of the output file. For example, the line command "converttwiss twiss_feb2000.asc btwiss.asc", takes the RHIC 
twiss file "twiss_feb2000.asc" eliminates the intermediate labels in this file and store the result in btwiss.asc.

\subsection{The Main Program}

There are two different main programs with names MODULAR.C or MODULAR_IR.C. MODULAR.C has been written to extract error values from the whole IR while MODULAR_IR.C has been written to extract error values from individual triplets. Both programs MODULAR.C and MODULAR_IR.C have 3 principal parts:

- Determination of average actions and phases in each arc of the accelerator and beam positions at the IR skew quadrupole corrector.

- Calculation of $\Delta x^{\prime}$ according to Eq. 3.4 and coefficients $a_{1}$ and $b_{1}$ according to Eq. 5.17.

- Screen printout of the above quantities for all the IRs.

The main core of the first item are the subroutines PRINCIPALX, PRINCIPALY, STRENGTHS_YELL and STRENGTHS_BLUE. These subroutines also contain smaller subroutines. All the subroutines will be described in Section 9.3.

The second item is the straight application of Eq. 3.4 and Eq. 5.17 to find $\Delta x^{\prime}$ and the $a_{1}$ and $b_{1}$ coefficients. All the values required for these Equations are produced in the first item. 
The third item is a for-loop that allows to print any of the variables evaluated in the first or the second item for all triplets in the ring if the program MODULAR_IR.C is used or for all IRs if the program MODULAR.C is used. The for-loop also print two files "delta_ir_h.dat" and "delta_ir_v.dat". Each file contain 2 columns for each triplet for a total of 24 for all 12 triplets. The 2 columns contain either the values $\Delta x^{\prime}$ and $x_{s k}$, the position of the beam at the corresponding skew quadrupole corrector or the values $\Delta y^{\prime}$ and $y_{s k}$ depending on whether the file is "delta_ir_h.dat" or "delta_ir_v.dat". These files are not created every time the program MODULAR.C or MODULAR_IR.C is run. Instead, every time the program is run a new line is added to this file. This allow to analyze the output of many orbits together.

\subsection{Subroutines}

\subsubsection{Subroutine GETBETA}

This subroutine read the twiss file produced by CONVERTTWISS to find the value of the beta functions and phase advance for a given value of the axial coordinate $s$. This subroutine has one input, the axial coordinate $s$, and four outputs, the beta functions and phase advances in both planes. The program gives values for the lattice function for any real value of $s$ even though the original twiss file only has the value of the lattice functions for a finite number of $s$ positions. In order to do that, the subroutine identifies two consecutive lines with $s$ values that are below and above the given $s$ value. The values of 
the lattice functions of those lines are then used to do linear interpolations.

\subsubsection{Subroutine GETPOSX and GETPOSY}

These subroutines are used to read either the horizontal (GETPOX) or the vertical (GETPOSY) beam position from an sdds orbit file in ASCII format. The subroutines read one orbit line whenever they are invoked and advance one line forward. The outputs are the value of the coordinate $s$ and its corresponding BPM measurement. The subroutines are also sensitive to the flag $f$ (see Section 9.1) which indicates if the BPM measurement of the orbit line is valid or no. GETPOSX and GETPOSY generate outputs only for valid lines. If they encounter a non valid line, they go forward one orbit line and evaluate the flag $f$ again. This process continues until a valid BPM measurement is found.

\subsubsection{Subroutine INITIATEPOS}

Orbit files usually contained long headers before the BPM data. In order to use the subroutines GETPOSX and GETPOSY it is neccesary to place the pointer of the file where the orbit data starts. This is precisely the function of the INITIATEPOS subroutine. INITIATEPOS reads the sdds file in ASCII format and advance where the first line of orbit data is. From this point the subroutines GETPOSX and GETPOSY can be used as many times as neccesary until the orbit file is completely read. 


\subsubsection{Subroutine PRINCIPALX and PRINCIPALY}

These subroutines are the central core of the program to find error from the RHIC orbits. A schematic diagram of the subroutines PRINCIPALX is shown in Fig. 9.1. The subroutine PRINCIPALX starts by setting two indices: $\operatorname{arc}$ which indicate the accelerator arc that is been processed and $n$, the number of BPMs in the mentioned arc. In the next step, the orbit file is read with the subroutine GETPOSX. This subroutine recover the current value of $s$, the axial coordinate along the ring, and its corresponding horizontal BPM reading. The subroutine GETPOSX is used twice in order to obtain the 2 adjacents BPM readings required by Eq. 2.2 to find the corresponding action and phase at a specific location in the ring. It is also neccesary to read the corresponding values of the lattice functions for the 2 adjacent positions. The subroutine GETBETAS is used twice to obtain the required beta functions. All the variables found in the previous steps like action, phase, lattice functions are saved in vectors with a length equal to the number of BPMs in the arc under analysis. This process is repeated until the end of the arc is reached. At the end of the arc the variable arc is incremented and the average values of action and phase in the arc are calculated according to values stored in the vector mentioned previously. Other quantities that are calculated at the end of the arc are the horizontal and vertical position of the beam at the position of the skew quadrupole correctors in the triplets by any of the 3 methods explained in Section 3.3. Also the vector $x \operatorname{xarc}[\mathrm{n}]$ can be fitted to the model using one 


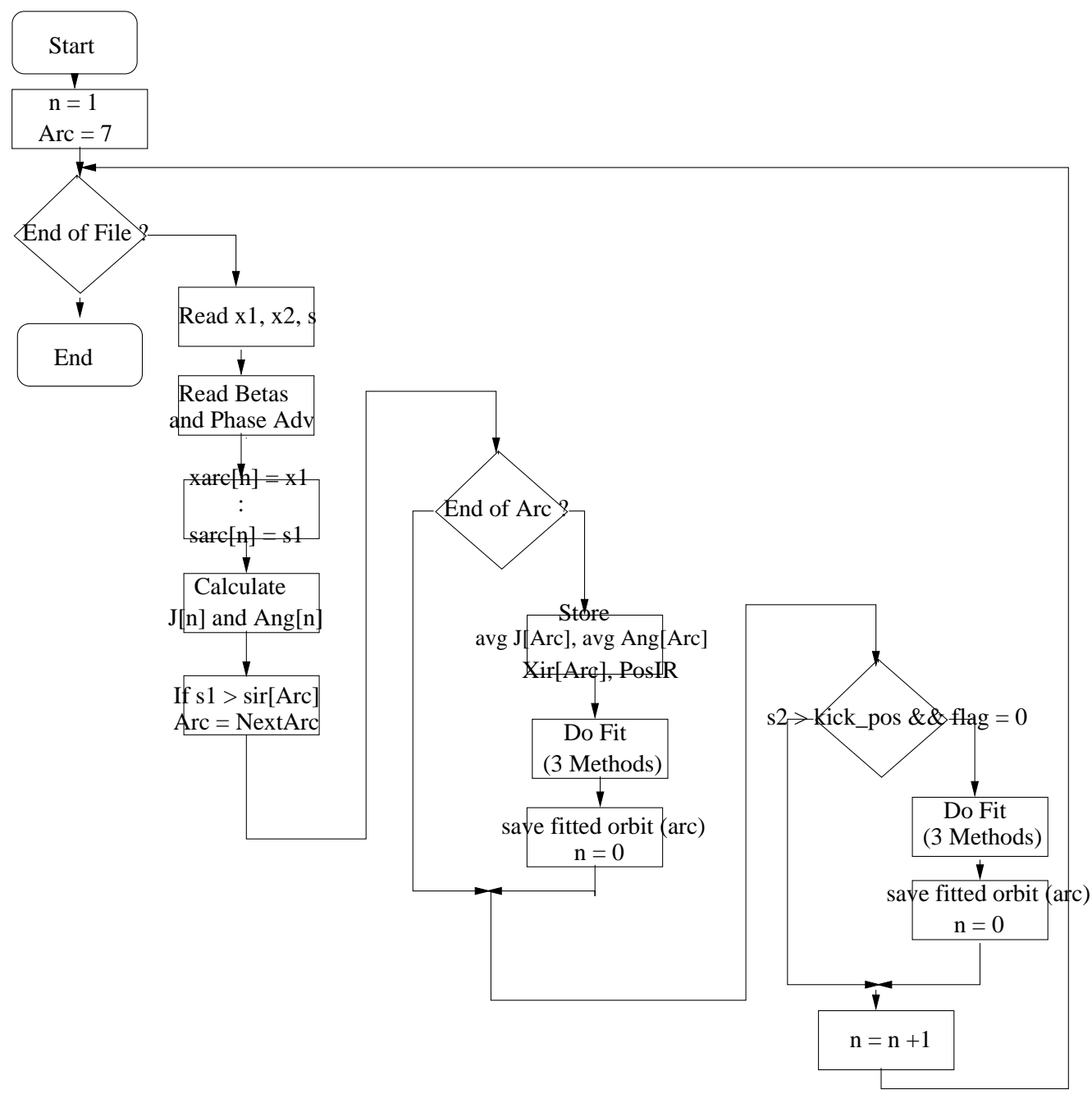

Figure 9.1: schematic diagram of the PRINCIPALX subroutine. PRINCIPALX and PRINCIPALY are the same except for the evaluation of the beta functions in each case. 


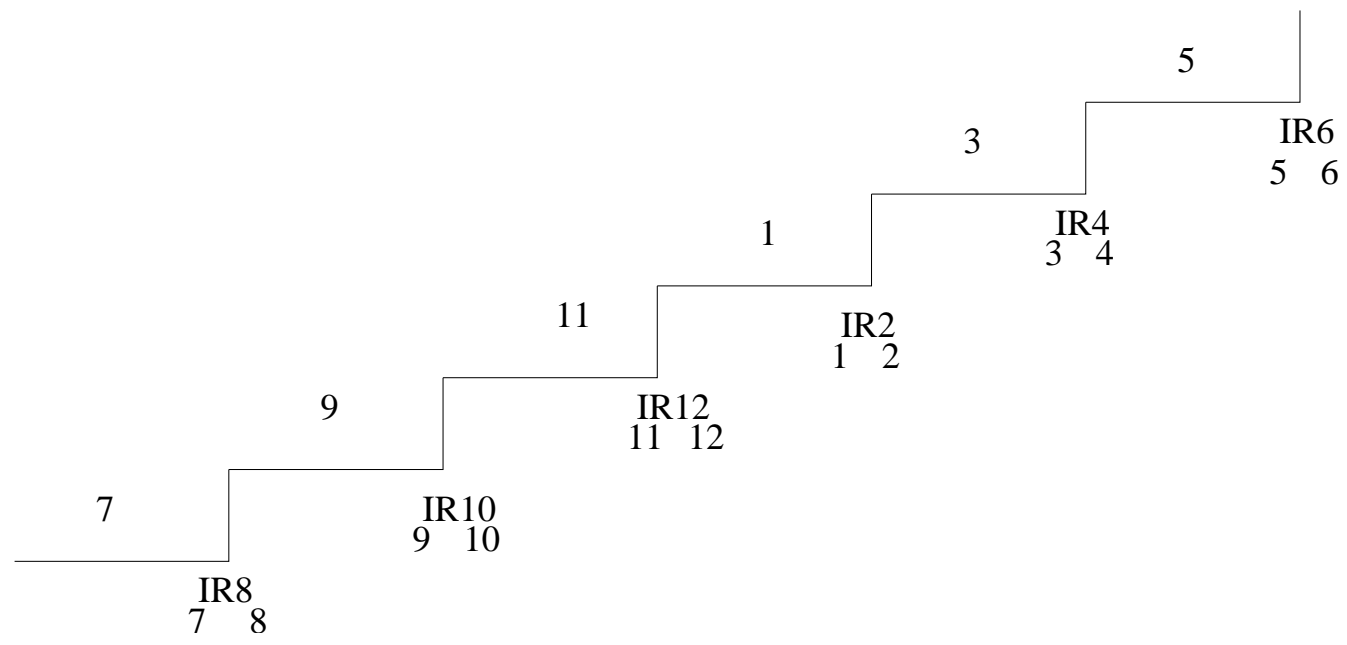

Figure 9.2: Convention used by the main program to label the arcs and the 2 sides of each RHIC IR. Every step represents and arc which is labeled with an odd number. It is also illustrated how each side of the IRs is labeled. Average action and phase in each arc as well as associated quantities are labeled with the number that corresponds to their arc. The same is valid for the quantities associated with the triplet like skew quadrupole corrector strengths and beam position measurements at the skew quadrupole corrector.

of the 3 different methods explained in Appendix C. The results of the fit are saved to a file that later can be retrieved to do comparisons with the real orbit. Finally the variable $n$ is returned to zero to prepare for analysis in the next arc.

In principle, the subroutine should produce 6 different values of average actions and phases, one for each arc. However, since the dipole kick used to either create the betatron oscillation in the case of the first turn trajectories or to shift the orbit in the case of the closed orbits, is present in the final difference orbit (see Fig. 2.2), the arc where the dipole corrector is must be 
divided in two parts. This division is performed within the last while-loop of Fig. 9.1 which basically selects the appropriate range of BPMs to be included in the calculation of average action and phase for the arc where the dipole corrector is located.

All the instructions described until now are repeated for any pair of adjacents BPMS until the end of the orbit file is reached. The most recent version of the subroutine has five inputs; plane of the kick to indicate if the dipole corrector was set in the horizontal or the vertical plane, position of the dipole corrector, name of the orbit file, position of all 12 skew quadrupole correctors and ring that is being analyzed either Blue or Yellow. The outputs are the averages actions and phases in six regions if the dipole corrector is in the opposite plane where the orbit is analyzed or seven regions if the dipole corrector is in the same plane in which the orbits are analyzed. Since the latest experiments will involve setting dipole correctors in both planes this subroutine is expected to be modified to always divide the ring in seven regions as discussed earlier. The other outputs are the beam position at the skew quadrupole correctors as well as the beta functions and phase advances. There is also an output called posirx_old that gives the value of the beam position at the skew quadrupole correctors but it is obtained in a different way than posirx. posirx_old is obtained using Eq. 3.9 while posirx_old is obtained by extrapolating the fit done in the previous arc with the subroutine BP_CORRECTOR (see Appendix C). 


\subsection{Auxiliar Software}

Several of the analysis performed in this thesis required additional software besides the MODULAR programs. The analysis performed in Section 5.5 and Chapter 7 , for example, require additional software to extract the values of the magnetic kick (either $\Delta x^{\prime}$ or $\Delta y^{\prime}$ ) as function of the beam position at a particular skew quadrupole corrector (either $x_{s c}$ or $y_{s c}$ ) from the delta_ir_h.dat and delta_ir_v.dat files. I have written a PERL program, EXTRACT_LIST and EXTRACT_LIST_IR, that takes the delta_ir_h.dat files and extract from them the column of delta values and beam positions for a given plane and triplet or IR and copy such values in another file with the name "ir_deltas.dat". For example, the command line "extract_list horizontal 8" read the columns $\Delta x$, $x_{s c}$ and $y_{s c}$ corresponding to the interaction region 8 and save such columns in the "ir_deltas.dat".

The procedure described in the previous paragraph usually involves a tedious process of typing at the LINUX prompt. In order to avoid this step, it is convenient to write a script that take all the orbits of the experiment and performed all the necessary steps to produce the "ir_deltas.dat" file. I have written PERL scripts that take all the orbits for a particular experiment, process all the orbits with either MODULAR.C or MODULAR_IR.C and create

the 3 columns file "ir_deltas.dat" for as many IR or triplets as desired. One example of such a script is "trip_bo3-th12.pl". This Perl script creates a shell script "trip_bo3-th12" that can be executed from a linux prompt. 


\section{Chapter 10}

\section{Concluding Remarks}

The development and application of a technique (action and phase analysis) to measure local errors at RHIC Interaction Regions, linear and nonlinear, has been presented in this first part of the dissertation.

Skew quadrupole errors at all RHIC Interaction Regions were found from the action and phase analysis on first turn difference orbits. These measurements were used successfully to locally correct for linear coupling in the machine.

The action and phase analysis were then generalized to closed orbits, where it was possible not only to extract skew quadrupole errors but also integrated gradient errors in some of the RHIC triplets with a precision that oscillate around $7 \%$. The error in the total gradient strength determination (no the error) can be as low as $0.01 \%$.

The skew quadrupole errors have their origin on roll quadrupoles. If the rolls associated with the quadrupoles (in this case quadrupoles of a RHIC triplet) are know then it is possible to estimate equivalent skew quadrupole 
errors associated with the triplet and comparisons can be done with the measured values. Roll angles of some of the RHIC triplets were measured during the 2002 RHIC shutdown period with this purpose in mind. There is a good agreement (within $15 \%$ ) between the calculated values with the measured angles and the measured skew quadrupole errors obtain from the action and phase analysis for most of the RHIC triplets considered.

The action and phase analysis was also extended to measure nonlinear errors specially sextupoles. The first results of these analysis on difference closed orbits give the correct order of magnitude for the expected sextupole components. We found that the order of magnitude of the statistical errors associated with the sextupole measurements is in some cases as big as the measurement itself but this can be improved by reasonable variations of the experiment.

Another set of interesting experiments were done by introducing know values of skew quadrupole errors, gradient errors and sextupole errors in the machine while trying to obtain their values with action and phase analysis. The correspondence between the measured values and the set values is around $5 \%$ for some of the experiments. Sextupole errors showed a clear relation between measured and set values. however, further analysis or experimentation is needed in this case to reduce the uncertainties associated with the sextupole measurements.

The fact that the action and phase analysis is specially useful at the interaction regions and allow to make very precise measurements at these 
locations is because the beta functions at the interaction regions are higher than anywhere else in the ring. In order to apply the action and phase analysis in the arcs and obtain the same precision as before in the error measurements one could intentionally raise the beta functions locally at the point of interest using for example beta bumps [57]. This is an interesting possibility to explore in the future that will expand the application of the action and phase analysis.

At the time this text is been written (May 2003), action and phase analysis with SPS (Super Proton Synchrotron at CERN) orbits are underway to determine the strength of known sextupole components are arbitrary locations of the ring [58]. Encouraging results have already found even before doing beta bumps. The action and phase analysis would combine in the future the advantages of methods like the response matrix to find errors all around the ring and the orbit bump method to find errors at localized places in the ring. The response matrix method calculates simultaneously all possible errors from a set of orbits obtained in one experiment while the orbit bump requires and experiment for every individual error. The action and phase analysis on the other hand, has the potential to determine from a set of orbits taken during one experiment, errors anywhere in the ring. The difference with the response matrix method in this case is that the errors are determined individually and they don't depend on each other. The amount of data proccessing in this case would be bigger than for the case of the response matrix method but this apparent drawback can be solved by well designed software. 


\section{Part II: Optical Design of the Rapid Cycling Medical Synchrotron}




\section{Chapter 11}

\section{Proton Therapy}

\section{$11.1 \quad$ Introduction}

Techniques like radiotherapy, chemotherapy, surgery and hadron therapy are used to treat a wide range of cancer manifestations. The most common techniques employed are radiotherapy and surgery or a combination of both. These techniques allow local tumor control which seems to be positively related with the chances of survival. Radiotherapy, however, has undesirable secondary effects that are difficult to minimize.

Hadron therapy unlike radiotherapy allows to irradiate cells very precisely, minimizing the irradiation of healthy tissue and hence the secondary effects that are common in radiotherapy. For this reason the use of hadron therapy, proton therapy in particular, is increasing specially in the last decade. During this interval of time, two dedicated proton facilities in the US and five in Japan have been commissioned and there are plans for many more in the future. 


\subsection{Physics of Proton Therapy}

When protons proceed through the tissue they cause ionization at the expense of their energy until they are finally stopped. R. Wilson was the first one to characterize this interaction in 1946 [34]. The dose depth distribution

shown in Fig. 11.1 is mainly determined by his first observations: "The dosage is proportional to the ionization per centimeter of path, or specific ionization, and this varies almost inversely with the energy of the proton. Thus the specifics ionization or dose is many times less where the proton enters the tissue at high energy than it is in the last centimeter of the path where the ionization is brought to a rest". The above properties make possible to irradiate within very precise boundaries a tumor located at certain depth under the skin.

The depth at which the protons are stopped or the range is a function of the incident energy of the protons as can be seen in Fig. 11.2. To reach most of the human organs the energy of the protons must vary between 70 $\mathrm{MeV}$ and $250 \mathrm{MeV}$. If it is necessary that the protons pass through the body before being stopped as it is required for some imaging techniques, the energy required must be bigger than $270 \mathrm{MeV}$.

Fig. 11.3 shows the dose depth distributions of electrons and X -Rays over imposed to the dose depth distribution of protons. The differences in these curves are mainly due to the different nature of the interactions of the different radiation with the tissue. While protons directly produce ionization, photons produces indirect ionization through process like photo-effect and pair produc- 


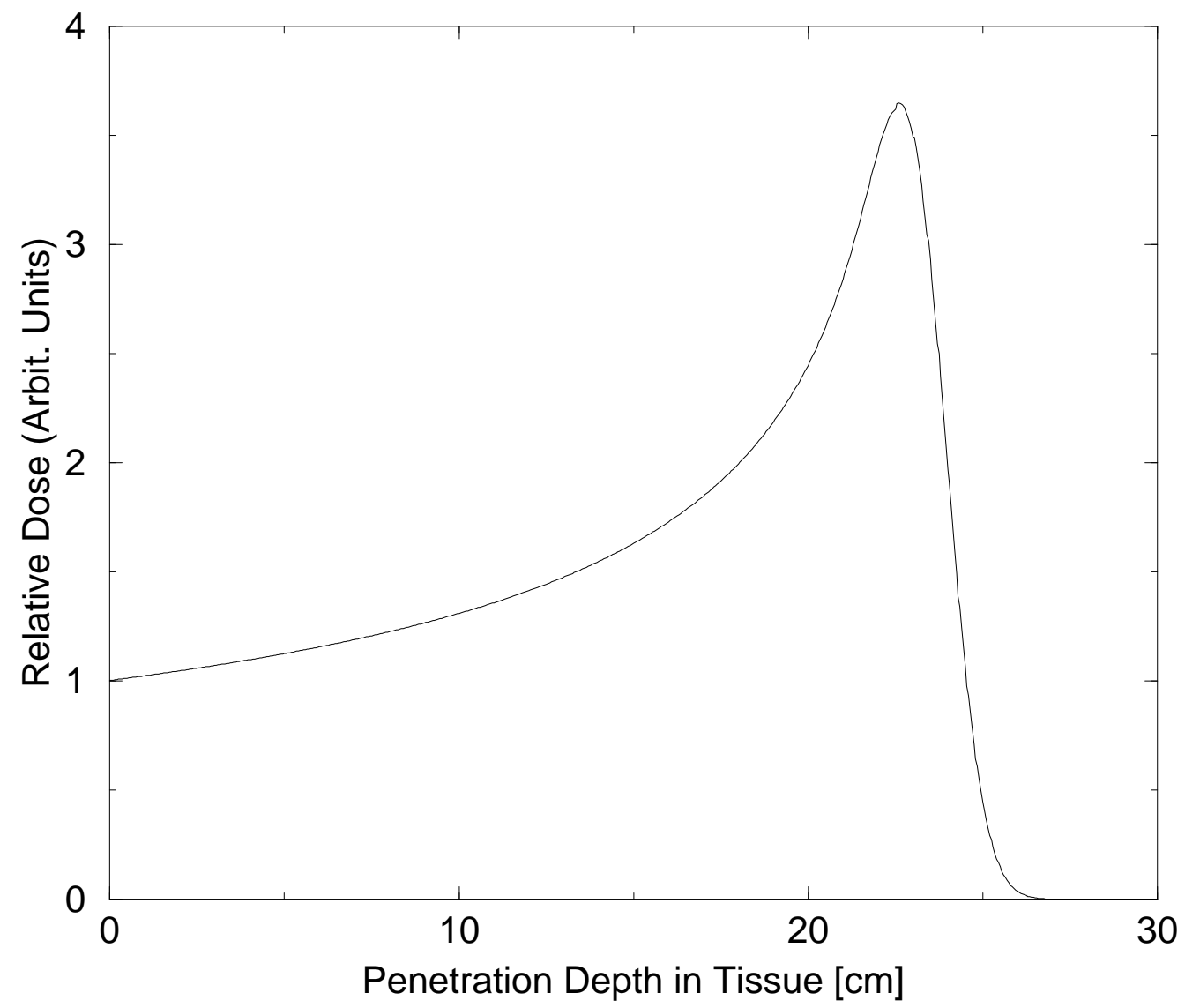

Figure 11.1: Dose deposition profile of a $190 \mathrm{Mev}$ proton beam in Tissue. The dose deposition near the skin is low compared to the deposition about $20 \mathrm{~cm}$ deeper. 


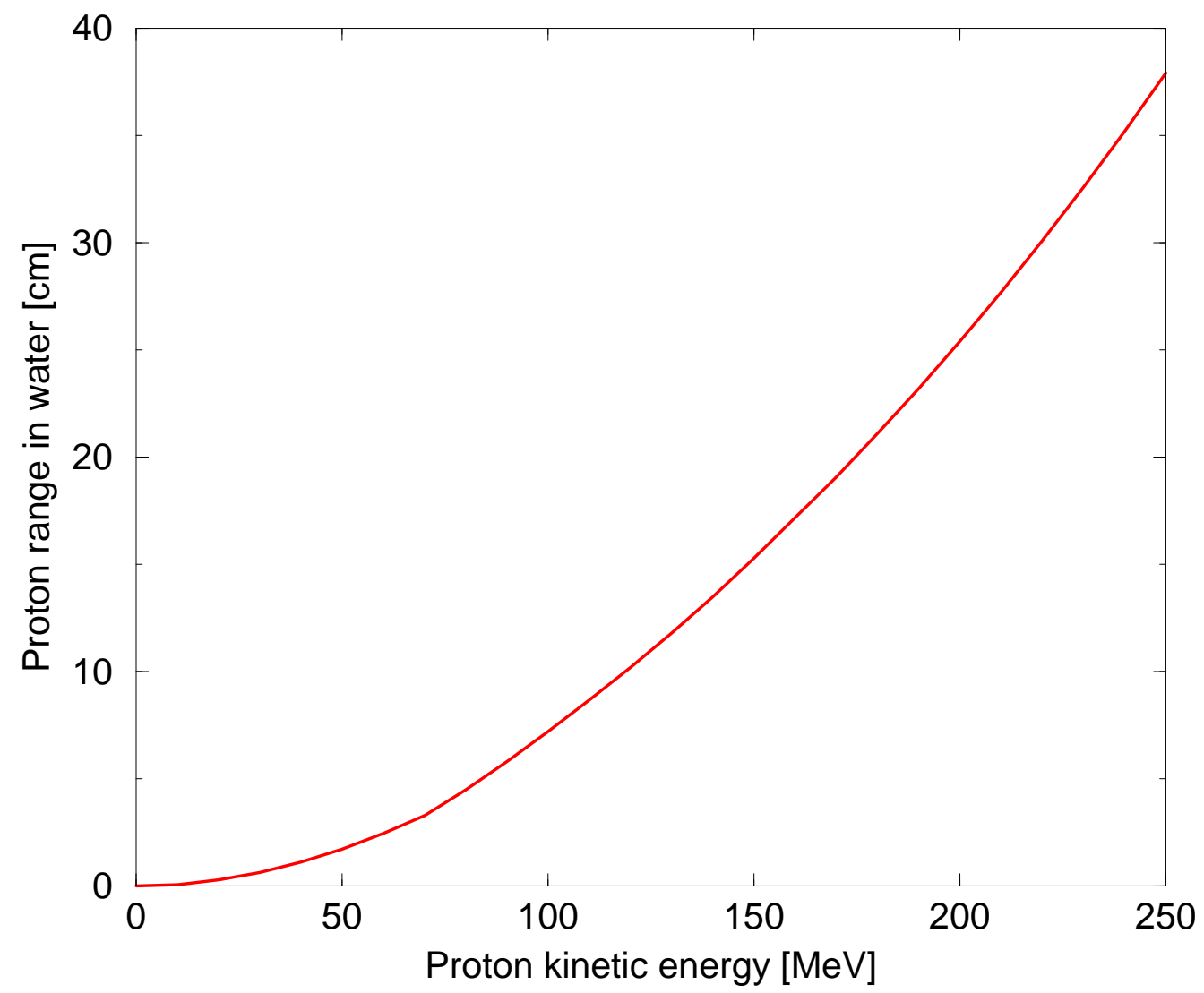

Figure 11.2: Range of protons in water as function of the incident proton kinetic energy. The behavior of the curve is almost linear in the range of interest. 


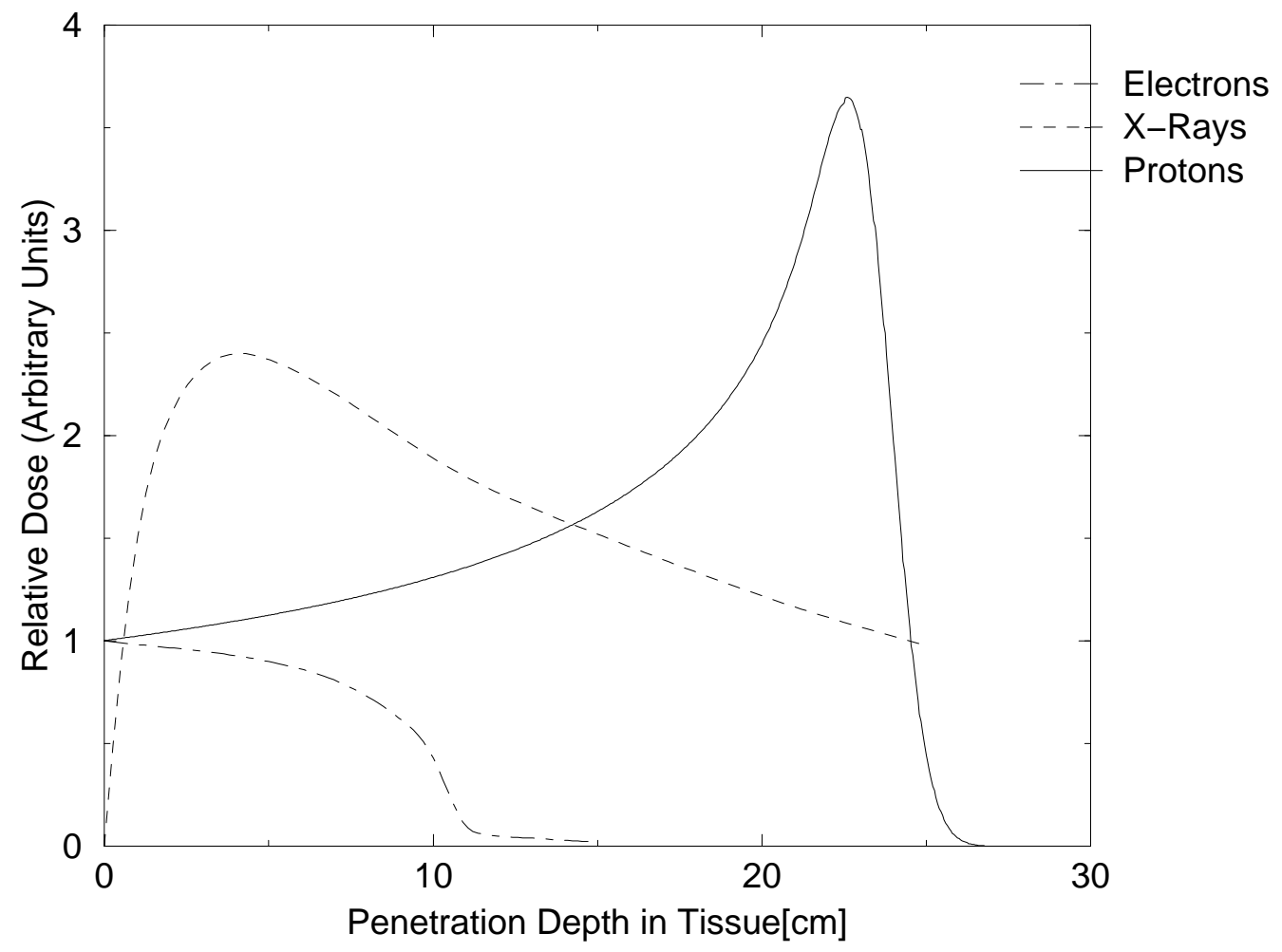

Figure 11.3: Dose deposition for three different types of radiation: Electrons, $\mathrm{X}$-Rays and protons. The proton dose deposition is much narrower than the other 2 types. 
tion. There is no energy lost of individual photons and hence the ionization cause by any photon at any depth has the same probability to occur. This leads to a exponentially decrease of the photon beam which means that most of the radiation will be absorbed by the tissue at the beginning of the photon path which is in agreement with the corresponding curve in Fig. 11.3. It is also worth it to mention that there is no abrupt stop of all the photons as in the protons case but they continuously decrease their intensity until they pass entirely the tissue.

From the above observations it is easy to see that radiotherapy with photons is more likely to act in healthy tissue than proton therapy. The broad peak of photon dose can be reduced by irradiating the tumor from different directions but even though proton dose distribution has a peak many times narrower than photon radiation.

Electrons also cause direct ionization as the protons do, but its range is more reduced due to the fact that electrons are many times lighter than protons and hence they are easily scattered. Electron radiation is useful only for superficial tumors or those just under the skin surface.

\subsection{Dosimetry of Proton Therapy}

In order to characterize the incoming radiation according to the energy that such radiation exchange with the tissue, the concept of Linear Energy Transfer (LET) was created. Photons can have LET around $0.3 \mathrm{Kev}$ per 
micrometer while LET for protons is in the range of $10 \mathrm{Kev}$ to $20 \mathrm{Kev}$ per micrometer.

Photons, protons, electrons and alpha particles are considered to have low LET. Neutrons and all other heavy ion are considered to have high LET. The reason for this division is related with the damage that the radiation causes in the tissue.

Radiation with low LET leave a pathway of spare ionization that causes minor damages to the cells (usually single break strands in the DNA structure) that the cells can repair itself. Particles with high LET in contrast, leave pathways with very dense ionization that can cause irreparable damage in the cells like double break of DNA strands.

Cancer cells usually lose their ability to repair themselves and a single break of their DNA seems to be enough to kill them. For this reason low LET radiation is preferred; it is enough to kill the cancer cells in one hand and on the other hand the effect in healthy tissue is repairable.

At the beginning of this chapter it was stated that hadron therapy has advantages over the conventional radiation therapy due to the fact that the dose distribution is more localized in the first one. Among the hadrons, the protons are the only ones with low LET. That make the protons very attractive for medical treatment and the particle of choice for the Rapid Cycling Medical Synchrotron. 


\section{Chapter 12}

\section{General Design Considerations for the Rapid Cycling Medical Synchrotron (RCMS)}

We will first present some of the requirements for a therapy accelerator because that will motivate the subsequent design choices. This discussion is an update of the original proposal of the RCMS done in FERMILAB in 1992 [35]. At that time, the facility was named Proton Therapy Accelerator (PTA). The project was then re-taken by BNL under the name of Rapid Cycling Medical Synchrotron or RCMS.

\subsection{General Requirements of a Therapy Fa- cility}

Besides the obvious requirement to deliver beam of the appropriate characteristics, preeminent design goals of a medical accelerator include reliability, economy, ease of maintenance, and patient safety. Contrast the design of high 
energy physics (HEP) research accelerators, where ultimate performance is commonly desired along with low capital cost. Beam users at a HEP laboratory are prepared to tolerate relatively frequent data taking interruptions, not only because that is the price of high energy performance but also because many technically skilled people are ready to effect repairs quickly. The manager of a medical radiation facility can not be so tolerant -only very infrequent rescheduling of patient treatment is acceptable and considerably fewer maintenance people are on site. Furthermore, although personnel safety is heavily emphasized at HEP facilities, additional safety measures are neccesary in the facility where patients are deliberately exposed to the beam.

The design of RCMS therefore emphasizes simplicity and modularity, for example by minimizing the number of different component types so that many parts are interchangeable. The RCMS design is also conservative in that the technologies adopted are well-establish by successfully use at existing accelerator. Furthermore, the design specifications are far from performance limits; the conservative methods used to estimate the performance limits have been validated by experience with other accelerators.

\subsection{Design Choices}

We believe that a synchrotron is the machine of choice for proton therapy. The most significant advantage over a linac or a cyclotron is that an appropriately designed synchrotron can straightforwardly produce a beam whose 
output energy is continuously variable within wide limits, a capability necessary for the beam delivery concepts to be described below. Linacs and cyclotrons can produce more intense beams, but well-designed synchrotrons can easily provide enough beam intensity for a therapy facility. The cost of a synchrotron is probably less than that of a linac and comparable to that of a cyclotron [36].

The proton synchrotron incorporates three features that are most important to achieving the design goals of the RCMS:

1. The Optical Lattice Design is based on the Strong Focusing Principle The magnet distribution or lattice includes regularly spaced quadrupoles that alternately focus and defocus the beam. This is the simplest realization of the alternating gradient concept, which results in strong net focusing in both transverse directions.

The optical design of the RCMS was made out FODO cells (see Chapter 14). These FODO cells might have dipole magnets between the quadrupoles (arc FODO cells) or they might be magnetically empty (straight section FODO cells). FODO optics are extremely simple and well behaved, with tight focusing that reduces the horizontal and vertical beam sizes, thereby reducing the cost of the magnets because their transverse sizes can be small.

2. The synchrotron is rapid-cycling and uses single turn extraction: Beam can be extracted from a synchrotron using single-turn fast extraction 
or resonant slow spill; fast extraction is preferable for several reasons. The minimal hardware and controls necessary to achieve single turn extraction lead to a simpler and more rugged system than that necessary to achieve slow extraction over thousand turns. Furthermore, the beam can be fast-extracted at arbitrary energy simply by changing the firing time of the extraction kicker; (of course the strengths of the extraction kicker, septum, and beam transport elements must be changed to track the beam momentum). Slow extraction inherently generates beam losses of order $1 \%$ or more, whereas fast extraction is inherently "clean". Unlike slow spill, fast extraction imposes no special requirements on the good-field aperture at extraction time. Painting the beam over a tumor volume during slow spill requires uniform spill; it is hard in practice to avoid fluctuations of the order of ten per cent in the spill rate of slowly extracted beam.

Fast extraction allows the choice of a rapid cycling synchrotron; slow spill takes so long that it effectively precludes rapid-cycling. A slow cycle essentially demands that a lot of protons occupy the accelerator simultaneously in order to achieve acceptable treatment times; slow extraction thus carries the threat of suddenly dumping a lot of beam into a localized region of a patient. A fast repetition rate allows the intensity requirements to be met with modest intensity per cycle, thereby eliminating intensity-dependent problems such as coherent instabilities 
and space-charge effects. It also allows scanning of large tumor volumes by delivering one beam bunch to each volume element or voxel, creating the desired dose distribution throughout the tumor while minimizing the dose delivered to normal tissue. This beam delivery method, which we call "pointillism", seems to be the most promising approach to realizing the full potential of proton therapy. Eros Pedroni and coworkers at PSI have developed a similar approach to beam delivery [37]. The high level of repeatability that comes with rapid cycling provides a natural way to ensure delivery of the intended number of protons on each cycle: if the accelerated beam falls outside tight intensity specifications, then it is trivial to reject that cycle and wait for the next, in order to continue a scan of the patient. In this sense the RCMS is a "digital" treatment accelerator. Finally, rapid cycling significantly reduces power costs, as shall be discussed below.

It is worth noting in passing that the $500 \mathrm{MeV}$ rapid cycling proton booster at the KEK high energy laboratory in Japan bears some similarities to the RCMS. For several years now it has used parasitic beam to treat a modest number of patients [38]

3. Beam is injected at a kinetic energy of $7 \mathrm{Mev}$ : The use of a relatively high injection energy bestows several advantages. It makes the protons more dynamically "rigid", significantly ameliorating the effect of the electrostatic "space charge" forces attempting to disrupt the bunch. 
In conjunction with the relatively small beam intensity per cycle, this means that the space charge effects are negligible. Also, since the beam shrinks adiabatically as it accelerates, a higher injection energy makes for a smaller beam, reducing the required synchrotron aperture. Higher injection momentum means stronger magnetic fields at injection time, reducing the effects of remanent fields. Finally a $7 \mathrm{MeV}$ linac can be used for parasitic radio-isotope production.

The kinetic energy of the beam extracted from the synchrotron will be between $70 \mathrm{MeV}$ and $250 \mathrm{MeV}$. A maximum energy of about 215-220 MeV is expected at the patient, if the beam is double scattered in the nozzle.

The facility will not be capable of proton radiography (unless the maximum energy is raised to about $270 \mathrm{MeV}$ ). An energy of $220 \mathrm{MeV}$ has a depth of penetration in tissue of approximately $30 \mathrm{~cm}$, an adequate depth for the treatment of most tumors.

The beam intensity will be about $3 \times 10^{12}$ protons per minute. Although this is small compared to the intensities achieved by some synchrotrons for high energy physics, still is not trivial to achieve in a small synchrotron with a low injection energy. The intensity requirement is "soft" in the sense that the highest intensities are needed only occasionally, mainly to treat large tumors in reasonable times (generally in exposure times of order a minute per visit), and the needs are sometimes inflated by allowing for various inefficiencies, especially the losses which inevitably accompany passive beam-spreading 
techniques. Nevertheless, the RCMS has been design to achieve a higher intensity, at least $3 \times 10^{12}$ protons per minute. The most important consequence of the high design intensity may be that no intensity dependence of the accelerator performance or beam sizes will be encountered in everyday operation as the intensity is adjusted over a range of values well below the ultimate performance limit. Higher intensities may also prove valuable for marginally shortening treatment times and/or for supplying beam to additional treatment rooms in the future; it would be folly to allow the intensity limitations of the accelerator to limit the size of the tumor that can be treated or the scope of the facility. In Table 12.1, we have added the emittance and the circumference of the synchrotron to the basic parameters of the RCMS already mentioned in this section.

\begin{tabular}{|c|c|c|}
\hline Maximum Extraction Energy & {$[\mathrm{MeV}]$} & 250 \\
Minimum Extraction Energy & {$[\mathrm{MeV}]$} & 70 \\
Injection Kinetic Energy & {$[\mathrm{MeV}]$} & 7 \\
Repetition Rate $f_{r e p}$ & {$[\mathrm{~Hz}]$} & 30 \\
Treatment protons per bunch N, min & & $1.0 \times 10^{7}$ \\
Treatment protons per bunch N, max & & $1.7 \times 10^{9}$ \\
Proton Flux R, max & {$[1 / \mathrm{min}]$} & $3.0 \times 10^{12}$ \\
Circumference C & {$[\mathrm{m}]$} & 28.6 \\
Normalized RMS emittance $\epsilon$ & {$[\mu \mathrm{m}]$} & 0.15 \\
\hline
\end{tabular}

Table 12.1: Primary parameters of the Rapid Cycling Medical Synchrotron 


\section{Chapter 13}

\section{IBEFUMFO: The Optics Design Strategy}

A typical problem in optics design is to match beam lines that have different lattice functions at the point where the two beam lines want to be joined. If such a difference is not corrected, the joined beam lines will readjust their lattice functions such that there will be an increase of the maximum beta function.

In some cases the growth of the beta functions is imperceptible and the overall performance of the whole beam line is not perturbed and hence correction is not needed.

When the growth of the beta functions is not tolerable, correction can be done, for example, by inserting quadrupoles between the 2 beam lines. The strengths of the quadrupoles and the distances between them are varied until the lattice functions are matched.

Fig. 13.1 is a simple example of the application of this method. In this 


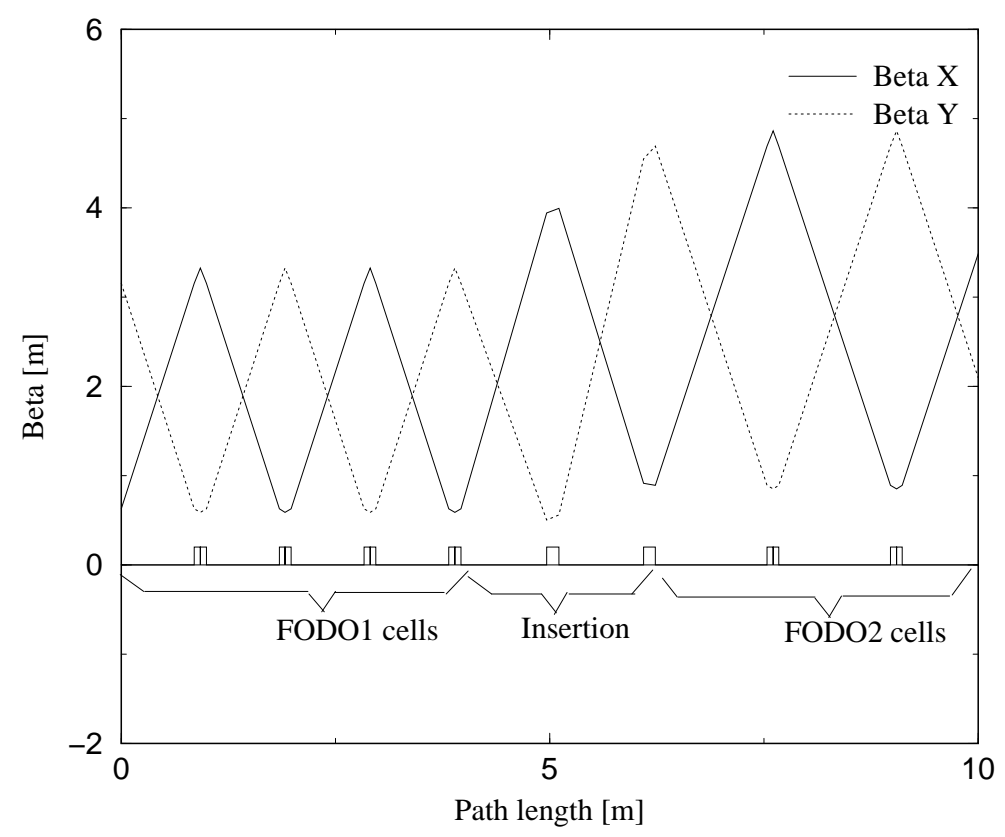

Figure 13.1: FODO cells structure of different lengths are matched by inserting two quadrupoles between them. (the narrow rectangles represent quadrupoles.) 
case, the beam lines to be matched are both made out of FODO cells with quadrupoles that have the same focal length but with different spacing between the quadrupoles.

The most common situation found in optical design is to have arbitrary incoming lattice functions that need to be matched to a FODO cell structure. In some cases the physical parameters (lengths and focusing strengths) of such FODO cells are known beforehand and hence the matching can be done by standard algorithms included in simulation software like MAD [10]. In other cases the physical parameters of the FODO cell are not known before the matching and the only restriction on the final FODO cell is the beam size or the maximum beta functions allowed. In this case it is convenient to develop relations that allow the physical parameters of the FODO cell to be found as function of such maximum beta functions. The matching process described in Fig. 13.1 using the mentioned relations is what we have named IBEFUMFO (Incoming BEta FUnction Matching to FOdo cells).

\subsection{The Basic Formulas of IBEFUMFO}

In the following lines I will deduced the relations between the physical parameters of the FODO cell and its maximum beta functions. It will be also neccesary to establish relations between the alpha functions of the FODO cell at the matching point and their corresponding beta functions. Assume a symmetric FODO cell like the one shown in Fig. 13.2. If the drifts have length 


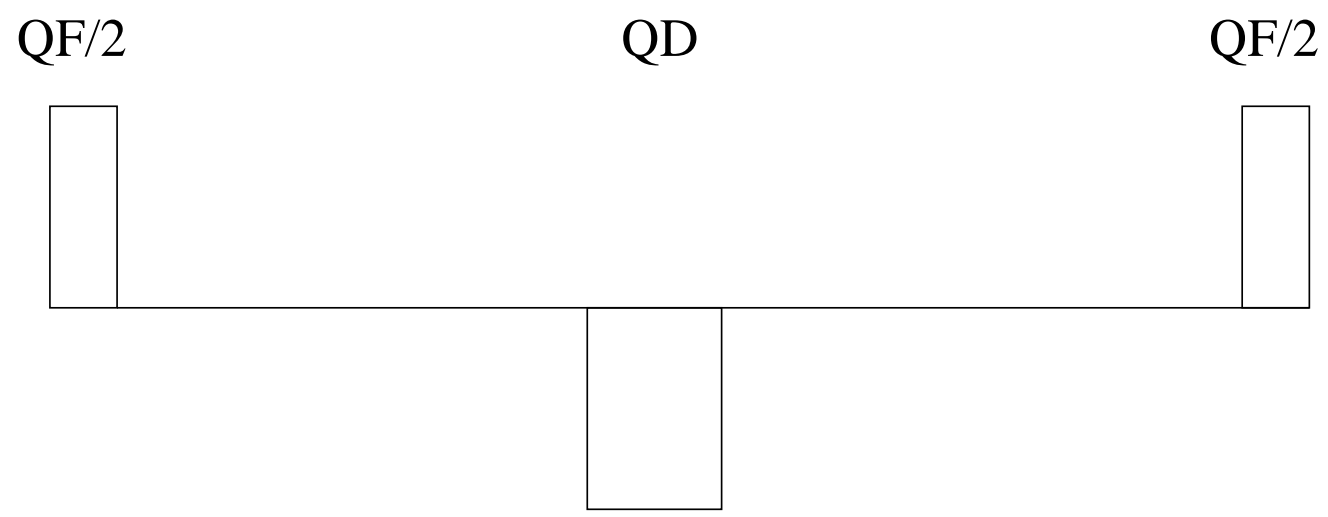

Figure 13.2: Symmetric FODO cell scheme made of a half defocusing quadrupole with focal length $\mathrm{f} / 2$, drift of Length $\mathrm{L}$, focusing quadrupole of focal length $\mathrm{L}$, and half defocusing quadrupole of focal length $\mathrm{f} / 2$.

$L$ and thin quadrupoles with focal length $f$ then the transfer matrix, M, of the FODO cell can be written as (see for example [39] pg. 48):

$$
M=\left(\begin{array}{cc}
1-\frac{L^{2}}{2 f^{2}} & L\left(2+\frac{L}{f}\right) \\
\frac{L(-2 f+L)}{4 f^{3}} & 1-\frac{L^{2}}{2 f^{2}}
\end{array}\right)
$$

Since the general form of matrix $M$ in function of the Courant - Snyder parameters is given by:

$$
M=\left(\begin{array}{cc}
\cos \Phi+\alpha \sin \Phi & \beta \sin \Phi \\
-\gamma \sin \Phi & \cos \Phi-\alpha \sin \Phi
\end{array}\right)
$$

then,

$$
\frac{1}{2} \operatorname{Trace}(M)=\cos \Phi=1-\frac{L^{2}}{2 f^{2}}
$$

or,

$$
\sin (\Phi / 2)=\frac{L}{2 f}
$$

Now comparing the second element in the first row of both matrices we get:

$$
\beta_{F}=\frac{2 L(1+\sin (\Phi / 2))}{\sin \Phi}
$$


To find the beta function in the center of the defocusing quadrupole the matrix $\mathrm{M}$ is defined starting at the center of the defocusing quadrupole of the FODO cell and the above procedure is repeated to obtain:

$$
\beta_{D}=\frac{2 L(1-\sin (\Phi / 2))}{\sin \Phi}
$$

Since one of the approaches that we follow for optical design is to choose the lattice functions (which are determined by the beam size) and then find the physical parameters of the lattice, it is necessary to invert the previous equations. Direct division of Eq. 13.5 and Eq. 13.6 lead to:

$$
\frac{\beta_{F}}{\beta_{D}}=\frac{1+\sin (\phi / 2)}{1-\sin (\phi / 2)} .
$$

Eq. 13.4 and Eq. 13.7 can be combined to obtain:

$$
\frac{L}{2 f}=\frac{\beta_{F}-\beta_{D}}{\beta_{F}+\beta_{D}} .
$$

Addition of Eq. 13.5 and Eq. 13.6 lead to:

$$
\beta_{F}+\beta_{D}=\frac{4 L}{\sin \Phi}=\frac{4 f}{\sqrt{1-\left(\frac{L}{2 f}\right)^{2}}}
$$

which trivially leads to:

$$
f=\frac{\left(\sqrt{1-\left(\frac{L}{2 f}\right)^{2}}\right)\left(\beta_{D}+\beta_{F}\right)}{4} .
$$

Eq. 13.8 and Eq. 13.10 can be combined to obtain:

$$
f=\sqrt{\beta_{F} \beta_{D}} / 2 .
$$


Multiplying Eq. 13.8 by twice Eq. 13.11, L as function of only beta functions can be found,

$$
L=\frac{\beta_{F}-\beta_{D}}{\beta_{F}+\beta_{D}} \sqrt{\beta_{F} \beta_{D}}
$$

Eq. 13.11 and Eq. 13.12 give the focal length and the length of the FODO cell as function of the maximum beta functions of FODO cell as was required initially.

Since all the physical parameters of the FODO cell are known at this point, the problem of doing the matching between a beam line and the FODO cell (see Fig. 13.1) can now be easily solved using programs like MAD. MAD, in particular, contains special commands that allow two beam lines to be matched through an optical insertion which in the case of Fig. 13.1 are the 2 quadrupoles separated by a drift.

Another possibility to solve the mentioned matching problem is to find a relation between the alpha and the beta functions at the matching point of the FODO cell. In this way, once the beta functions are given all the lattice functions will be known at the matching point. The inserted quadrupoles can then be varied to match the lattice functions at the entrance of the FODO cell. The physical parameters of the FODO cell can be found by Eq. 13.11 and Eq. 13.12 as before or by periodically matching the FODO cell to the known values of the lattice functions at the entrance of the FODO cell.

In order to find the relations between the beta and alpha functions it is now convenient to relate $\beta_{D}$ and $\beta_{F}$ with the lattice functions at one particular 


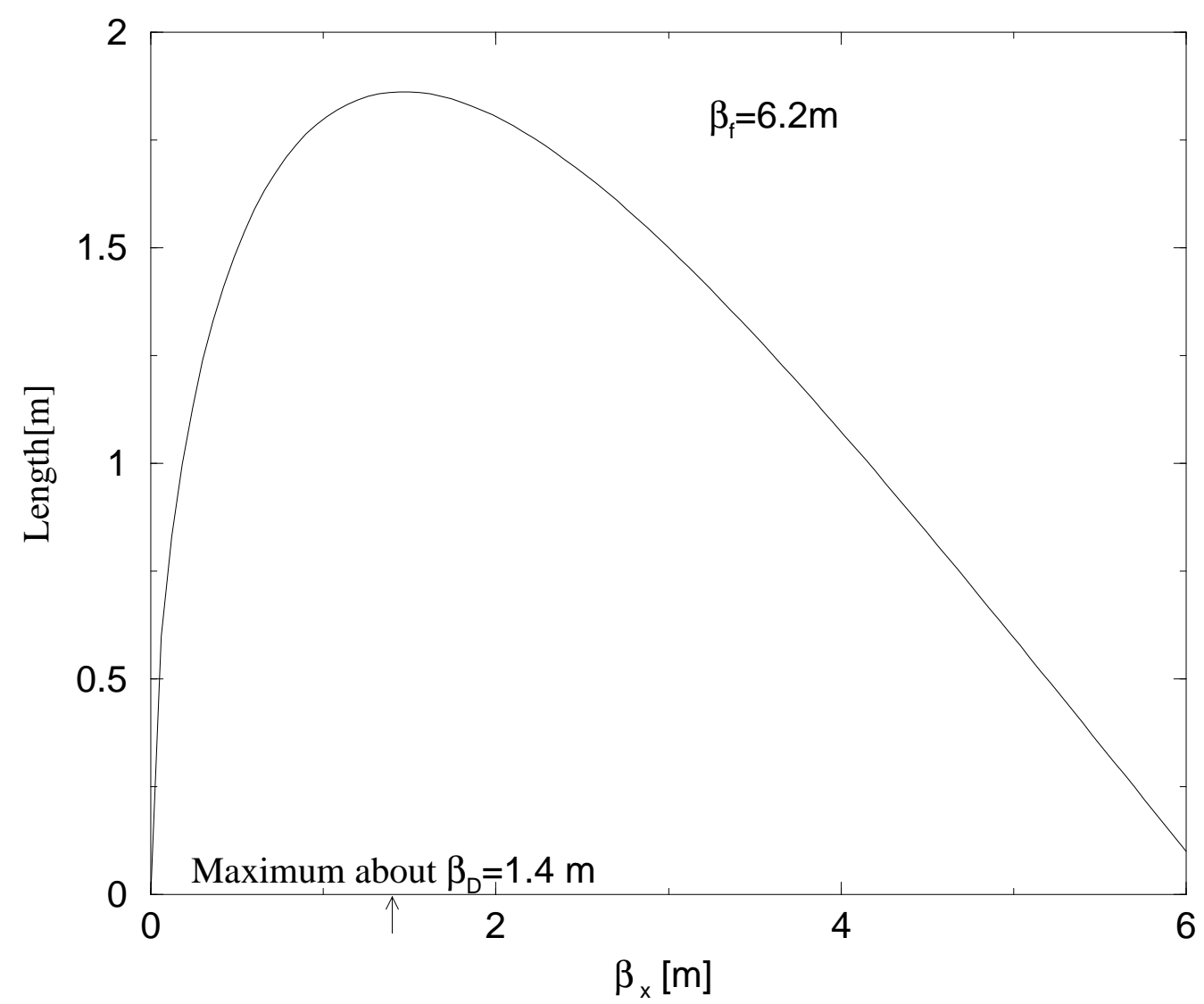

Figure 13.3: The inter-space between quadrupoles of a FODO cell has a maximum for some value of the minimum beta function in the FODO cell 
position in the lattice. Since a defocusing quadrupole in the horizontal plane is also a focusing quadrupole in the vertical plane the beta functions at the center of the quadrupole $\left(s_{0}\right)$ should be given by:

$$
\begin{aligned}
& \beta_{x}\left(s_{0}\right)=\beta_{D} \\
& \beta_{y}\left(s_{0}\right)=\beta_{F}
\end{aligned}
$$

At this position the alpha functions are zero since this place is a turning point for the beta functions. Rather than having the lattice functions at the center of the quadrupole it is neccesary to have them at one side of the quadrupole, the real position where the matching will take place. These lattice functions can be calculated with the evolution equations (Eq. 2.56 in [39]) for a thin quadrupole,

$$
\begin{aligned}
& \beta_{2}=\beta_{1} \\
& \alpha_{2}=\alpha_{1}+\beta_{1} / f,
\end{aligned}
$$

where subscript 1 labels lattice functions before the quadrupole and subscript 2 labels lattice functions after the quadrupole.

According to Eq. 13.14 the beta functions at either side of the quadrupole are the same as in the center. The alpha functions, on the other hand, change when going from position 1 to position 2. Since alpha at the center of the quadrupole is zero it immediately follows that the alpha functions at either 
side of the quadrupole are:

$$
\alpha_{2}= \pm \frac{\beta_{1}}{2 f}
$$

where the sign depends on the kind of quadrupole considered (either focusing or defocusing) and also the side of the quadrupole where the alpha function is evaluated. For example, the alpha functions at the left hand side of defocusing quadrupole can be found by substituting Eq. 13.11 in Eq. 13.15 leading to:

$$
\begin{aligned}
& \alpha_{y}=\frac{\sqrt{\beta_{y}}}{\sqrt{\beta_{x}}} \\
& \alpha_{x}=-\frac{\sqrt{\beta_{x}}}{\sqrt{\beta_{y}}} .
\end{aligned}
$$

It is also possible to put some other conditions in the design besides the beam size, like for example maximizing the length of the FODO cell from Eq. 13.12. In such a case, once the maximum beta function is specified the corresponding minimum beta function will be determined (see Fig. 13.3).

This approach in which Eq. 13.16 is used in combination with MAD numerical matching were one the most important tools in the optimization of the optical design of the RCMS. 


\section{Chapter 14}

\section{Lattice Design of the RCMS}

The design of all beam lines of the RCMS are based on the strong focusing principle. The advantages of this design choice are small beam sizes modularity and simplicity. Small beam sizes also imply smaller and lighter magnets which in turn reduce costs.

Since dispersion contributes significantly to the beam size every effort has to be made in order to suppress or reduce dispersion anywhere in the ring. Dispersion in the straight sections of the RCMS ring has been made equal to zero and minimized in the ring arcs. Special care has been taken in compensating locally sources of dispersion in the delivery system.

The ring is made out of FODO cells, in the arcs as well as in the straight sections with the last ones having longer lengths in order to accommodate the injection system, the extraction system and the instrumentation.

The tunes of the machine were also taken into account during the design. They have to be as far as possible from the stronger resonances.

In the following sections, a detail description of the optical design of the 


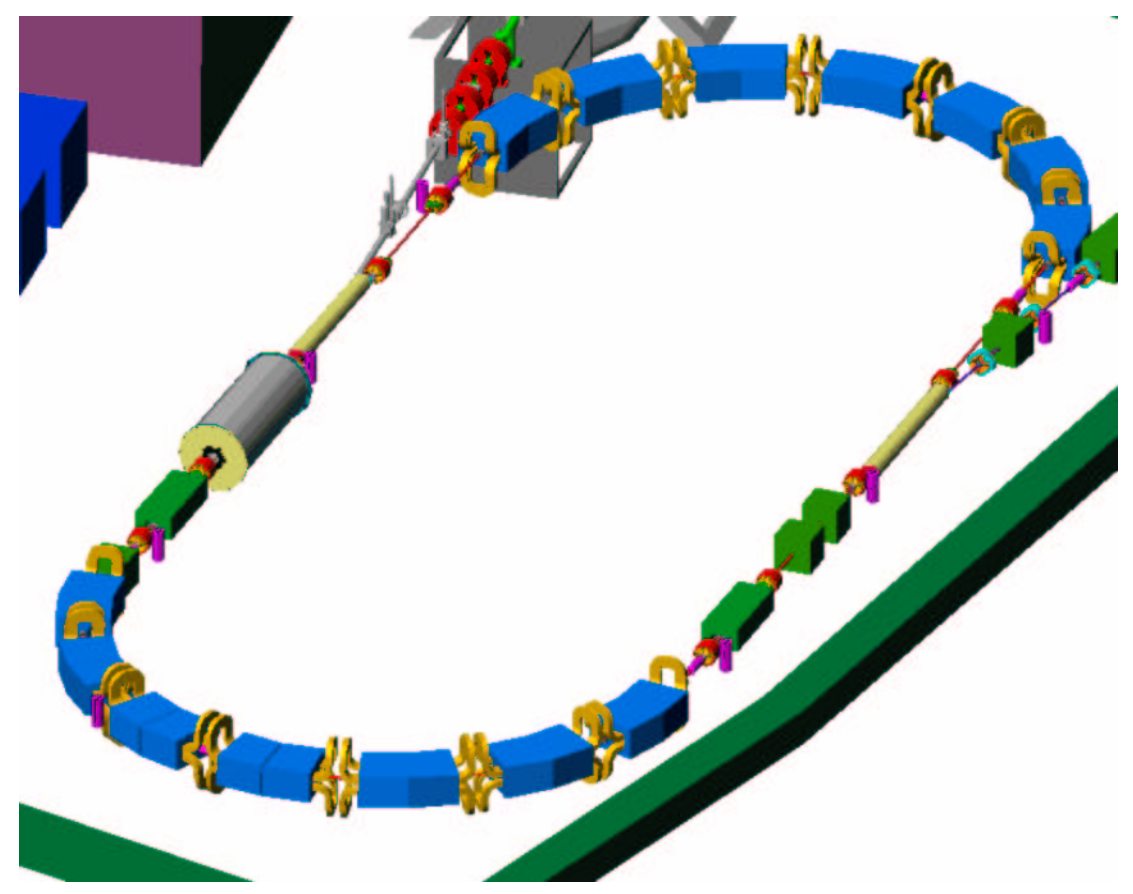

Figure 14.1: Perspective view of the racetrack layout of the synchrotron.

RCMS is given.

\subsection{Ring Optics Design}

The synchrotron ring has two arcs and two straight sections (see Fig. 14.1 and Fig. 14.2). The arcs are made out of FODO cell structures with a half length equal to $1.1 \mathrm{~m}$ which guarantees small beam sizes. These FODO cells are arrays of alternate focusing and defocusing combined function magnets which are dipoles with the poles slightly tilted to add a quadrupole component to the magnets.

Since the maximum magnetic field allowed for the dipoles is $B=1.4 T$ 


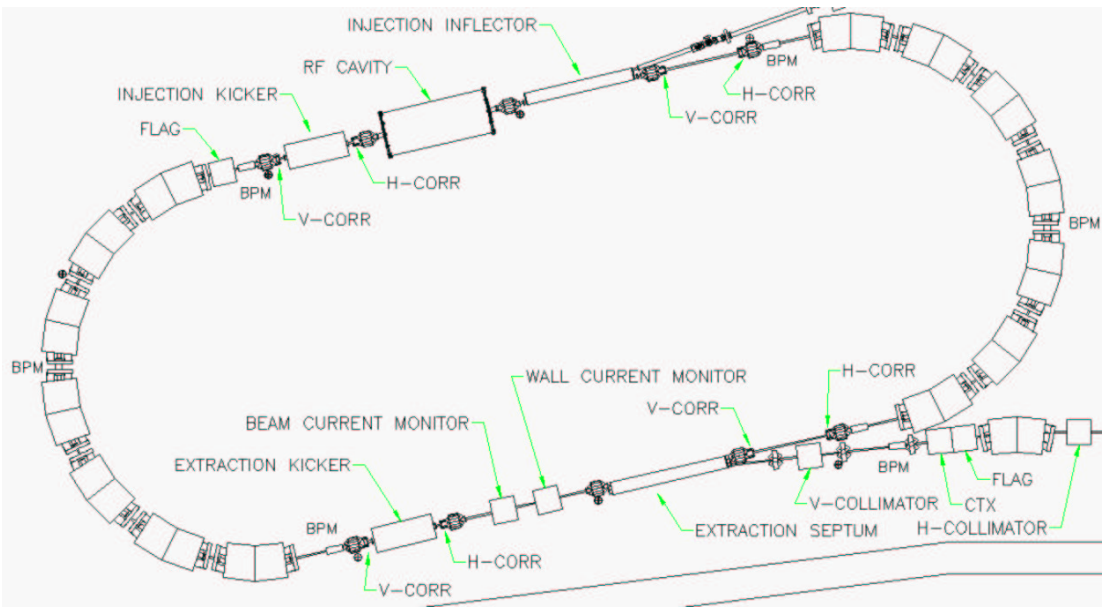

Figure 14.2: Plan view of the racetrack layout of the synchrotron.

and the rigidity of the beam at maximum energy is $B \rho=2.4 T m$, the total length of iron needed in each arc to bend the trajectory 180 degrees according to Eq. 14.1 is about $5.46 \mathrm{~m}$,

$$
S=\pi \frac{B \rho}{B}
$$

This iron length has to be distributed between the $1.1 \mathrm{~m}$ spaces defined by the FODO cells. Table 14.1 shows 4 different possible configurations. The 5 magnet configuration barely leaves any space between adjacent magnets. The 6 magnet configuration leaves about $10 \mathrm{~cm}$ between adjacent magnets. However, the 7 dipoles configuration (bending angle equal to $25.71^{\circ}$ ) was chosen instead of the 6 dipoles configuration because the first one lead to a symmetric lattice. This means that the lattice functions in the 2 arcs will be identical as well as the lattice functions in the 2 straight sections. This feature will be very convenient since it will significantly help to reduce the number of power supplies needed 


\begin{tabular}{|c|c|}
\hline No. Magnet & $\begin{array}{c}\text { Magnet Length } \\
{[\mathrm{m}]}\end{array}$ \\
\hline 5 & 1.091 \\
6 & 0.910 \\
7 & 0.780 \\
8 & 0.682 \\
\hline
\end{tabular}

Table 14.1: Relation between the magnets needed in the arc and their lengths to always produce a total bend of 180 degrees with the same magnetic field

for the matching process that will be described later.

Dispersion is made equal to zero outside the arcs by adjusting the strength of the focusing quadrupole component of the combined function magnets. However, after the dispersion matching, the horizontal and vertical beta functions significantly differ from each other leading to bigger beam sizes. The defocusing strength and the edge angle of the combined function magnets are then used to bring together the horizontal and the vertical beta functions again. The resultant beta functions can be seen in Fig. 14.3

The straight sections should have inter-spaces between the quadrupoles long enough to hold the instrumentation and the accessory components. In particular, it is desirable to have a slot for the RF cavities and extraction septum longer than the distance between the centers of two adjacent combined functions magnets in the arcs. This unavoidably leads to an overall change in the beta functions around the ring unless special matching conditions are imposed in the border of the straight sections and the arcs.

The IBEFUMFO algorithm described in Chapter 13 can be used in this case to optimally perform the required matching. As stated in Chapter 13, 


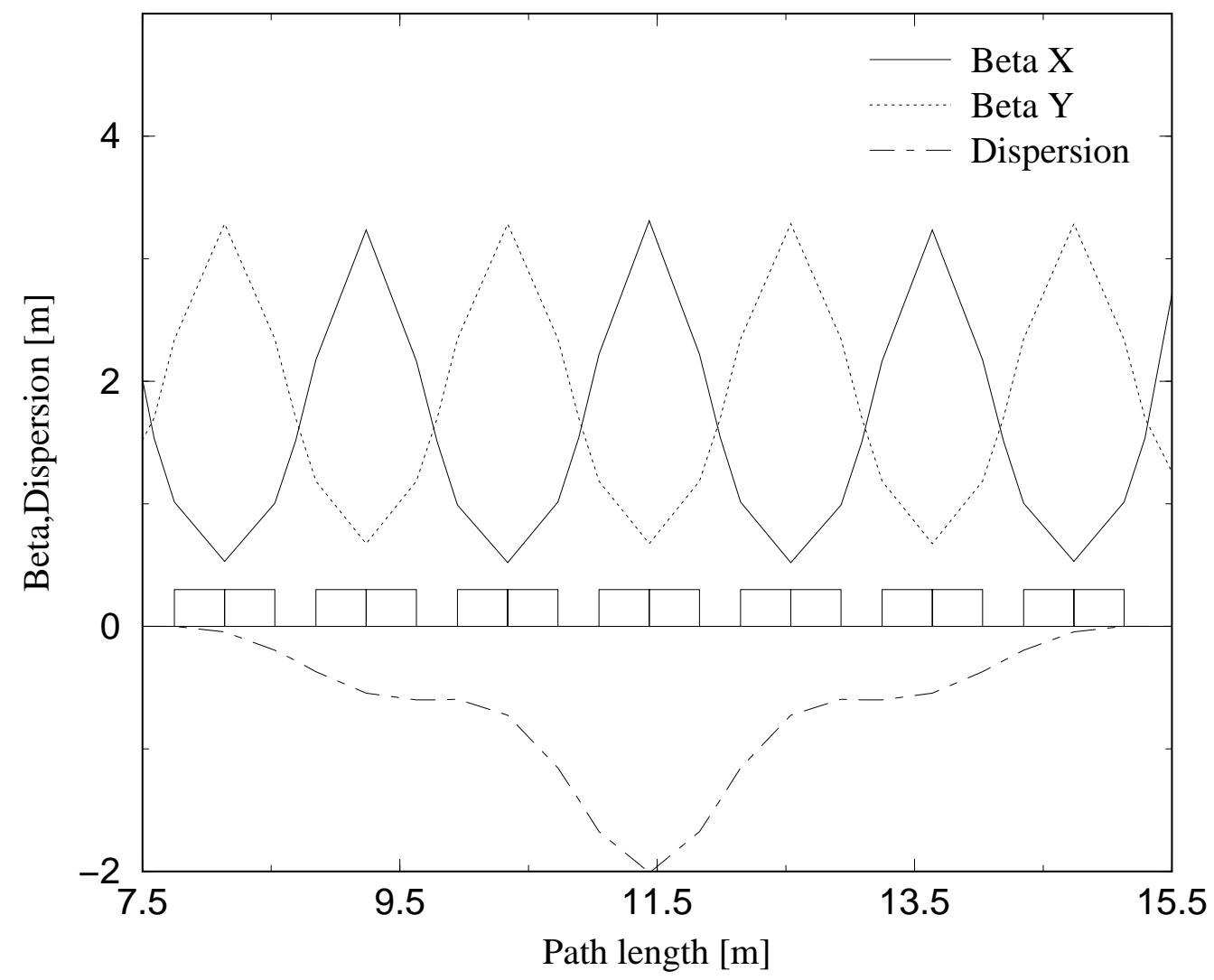

Figure 14.3: The arc is made out of 7 dipole which lead to a lattice with reflexion symmetry about the center of the arc.

\begin{tabular}{|l|r|}
\hline$\beta_{x}[\mathrm{~m}]$ & 1.00 \\
$\beta_{y}[\mathrm{~m}]$ & 6.10 \\
$\alpha_{x}$ & -0.40 \\
$\alpha_{y}$ & 2.47 \\
$L[\mathrm{~m}]$ & 1.68 \\
$F[\mathrm{~m}]$ & 1.20 \\
\hline
\end{tabular}

Table 14.2: Basic matching parameters in the straight section of the RCMS ring. 
IBEFUMFO uses two quadrupoles to do the matching. The first two quadrupoles of the straight section constitute the two required quadrupoles. The central quadrupole of the straight section and the spaces at each side of this quadrupole constitute the final FODO cell structure (see Fig. 14.4 and Fig. 13.1).

The IBEFUMFO algorithm requires two input values: the horizontal and vertical beta functions, $\beta_{x}$ and $\beta_{y}$, at the common point between the matching quadrupoles and the FODO2 cell in the straight section. The corresponding alpha functions, $\alpha_{x}$ and $\alpha_{y}$, are calculated with Eq. 13.16. The length and the focal length of the FODO2 cell are found by demanding the beta functions to be equal at the entrance and exit of the FODO cell and equal to $\beta_{y}$ and $\beta_{x}$. Table 14.2 show all the basic parameters used in the matching process described.

Since arcs and straight sections have mirror symmetry the matching done on one side is identical to the match required on the other side. Also, the straight sections are identical which means that the same power supplies can be used for both straight sections. Only three different quadrupole power supplies are required to do the matching in the whole ring.

Notice that the solution provided by the IBEFUMFO algorithm avoids any increase of the beta functions in the arcs and leads to an acceptable increase of the beta functions in the straight sections (see Fig. 14.5). It is even possible to do the match such that the maximum beta function in the arcs is the minimum possible for a given FODO cell of length $L$. In such a case only the $\beta_{y}$ can be chosen. $\beta_{x}$ is determined by Eq. 13.12 as the value 
that makes the length $L$ a maximum.

\subsection{Injection}

The nominal injection trajectory is shown in Fig. 14.10. Here we have

$$
\phi=6.5^{\circ}, \quad \psi=-M_{11} X_{I} / M_{12}
$$

where $X_{I}=a+h=18.3 \mathrm{~mm}$ and $M_{i j}$ are the elements of the transfer matrix from the inflector exit to the injection kicker. We also have

$$
D=\rho \sin \phi, \quad d=\rho \sin \psi,
$$

where $D+d=1.3809 \mathrm{~m}$. The radius of curvature is then

$$
\rho=\frac{D+d}{(\sin \phi+\sin \psi)}
$$

and

$$
\begin{gathered}
L=\rho \tan (\phi / 2), \quad H=L \sin \phi, \\
l=\rho \tan (\psi / 2), \quad h=l \sin \psi, \quad a=X_{I}-h .
\end{gathered}
$$

Fig. 14.6 shows a general view of the injection and extraction interfaces from the tandem Van de Graaf injector (see [54]), and into the switchyard that serves the treatment rooms. Fig. 14.7 shows some perspective detail of the injection interface with the incoming beam merging with the circulating beam. While the incoming beam is always in the same horizontal plane as 


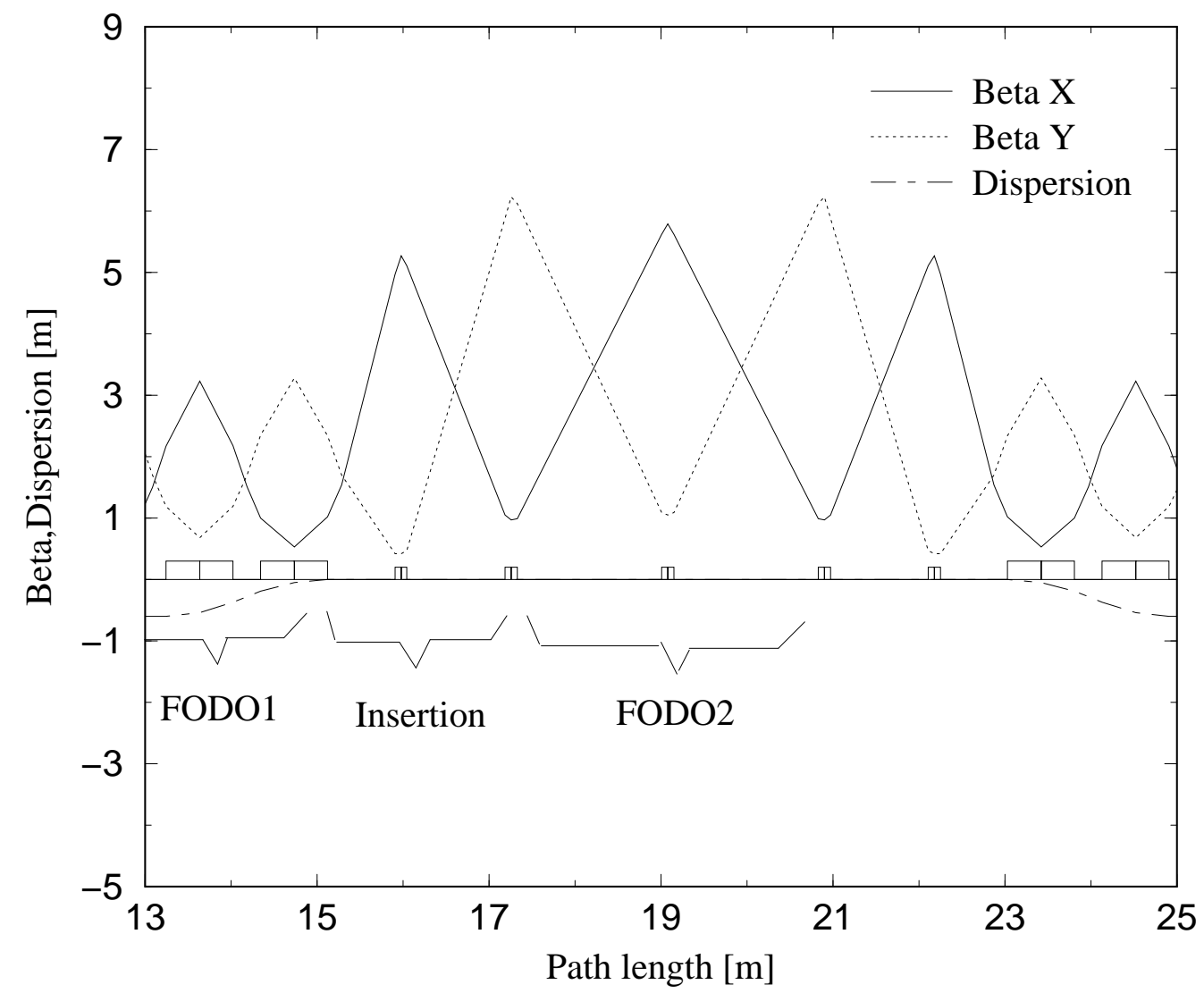

Figure 14.4: The application of IBEFUMFO easily allows to increase the length of the FODO cells in the straight section while keeping small the beta functions in the arcs. (the arcs can be identified by the wide rectangles that represent dipoles) 


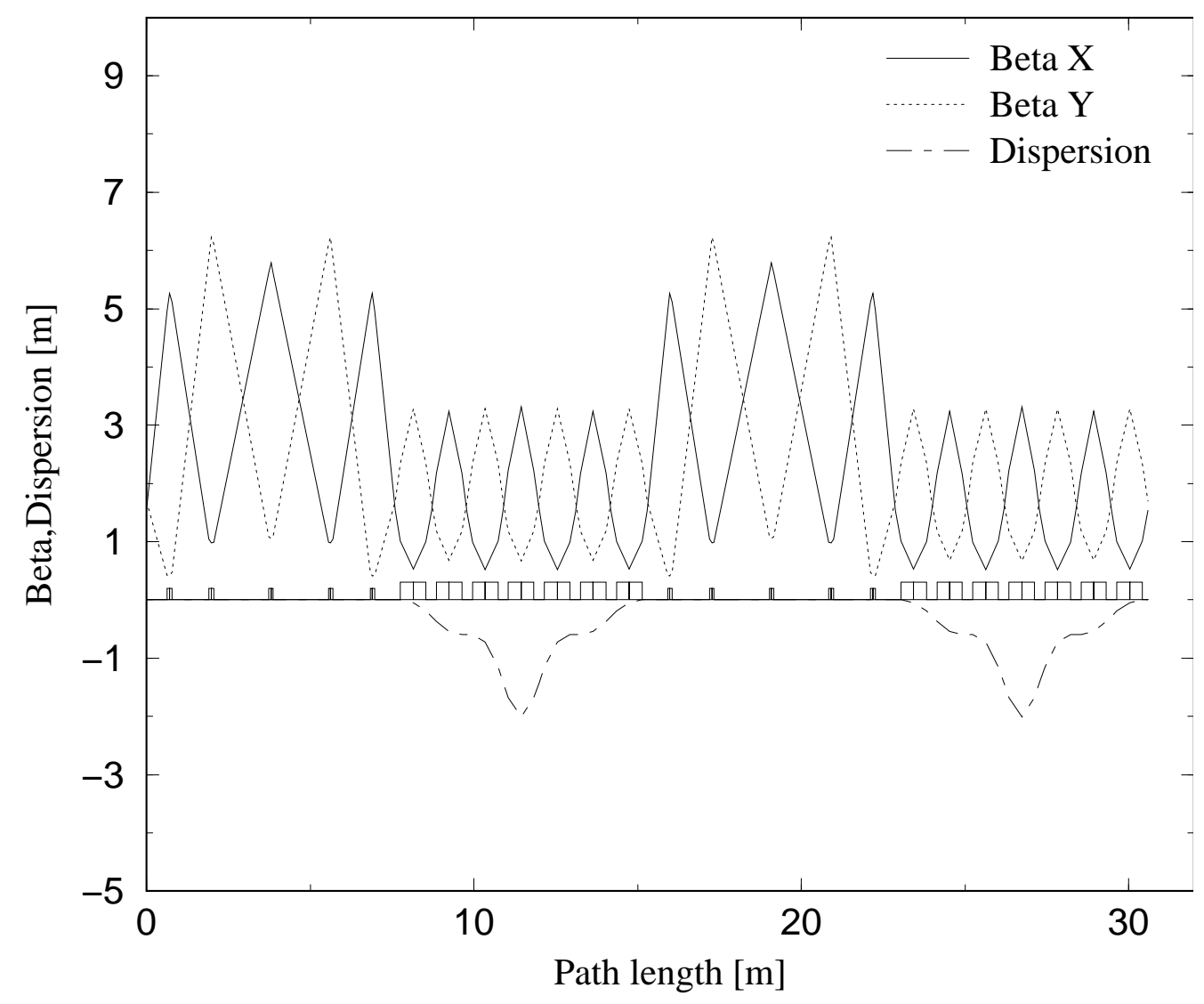

Figure 14.5: Lattice functions for the whole RCMS ring. 


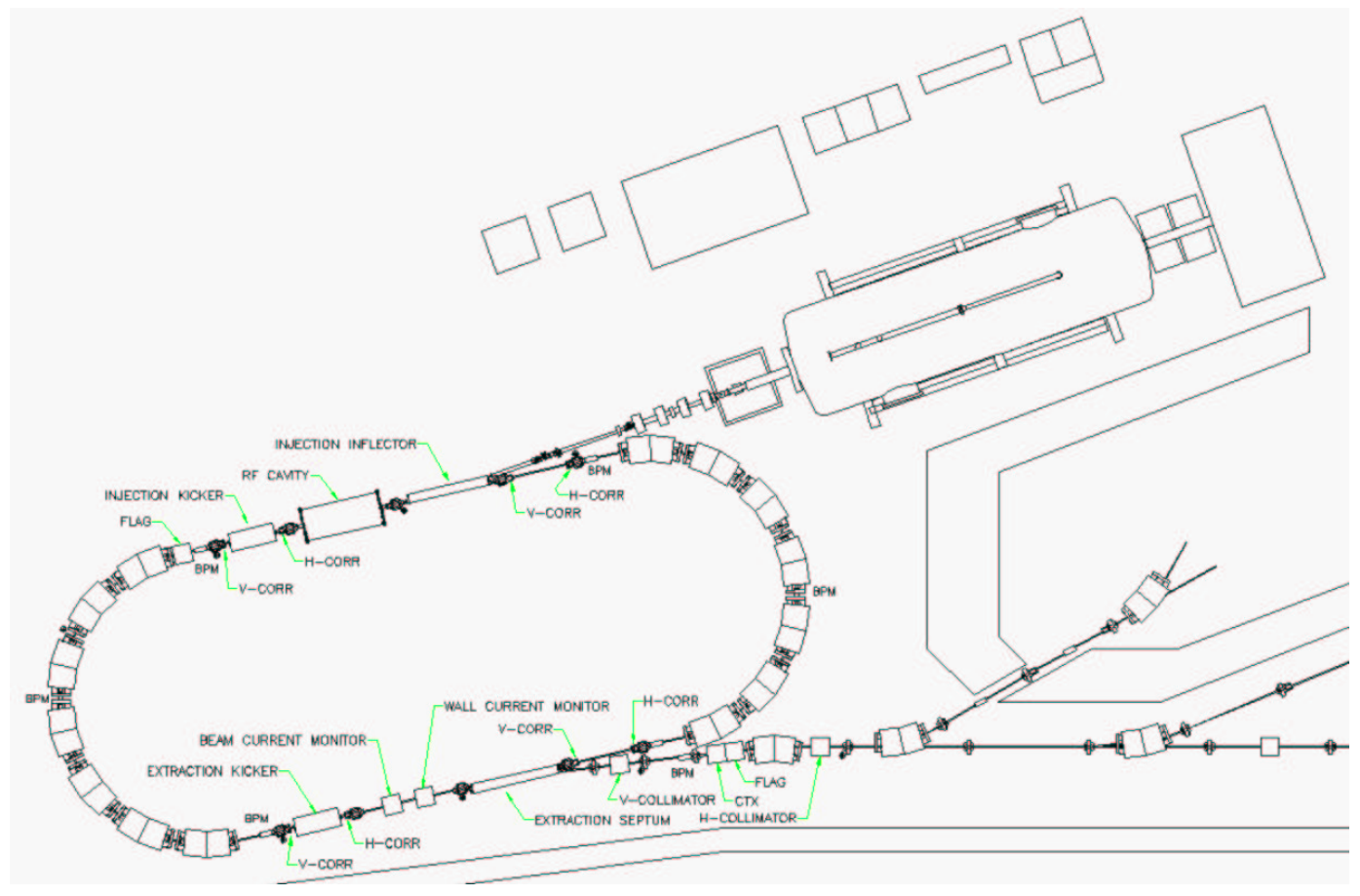

Figure 14.6: Plan drawing of the synchrotron, showing a broad view of the injection interface with the tandem Van de Graaf injector, and the extraction interface with the first few switchyard elements.

the circulating beam, the horizontal angle and displacement between the two must be reduced to zero. This is the function of the electrostatic inflector and the injection kicker, shown schematically, and in plan detail, in Fig. 14.8 and Fig. 14.9. Most of the work is done by the inflector, a simple device with a constant electrostatic field. At the end of the inflector both beams are in the same beampipe for the first time. The injection kicker - a pulsed magnet finishes the job.

The scale drawing of the electrostatic inflector in Fig. 14.11, and the sketch of the apertures at the downstream end of the inflector in Fig. 14.12, show a 


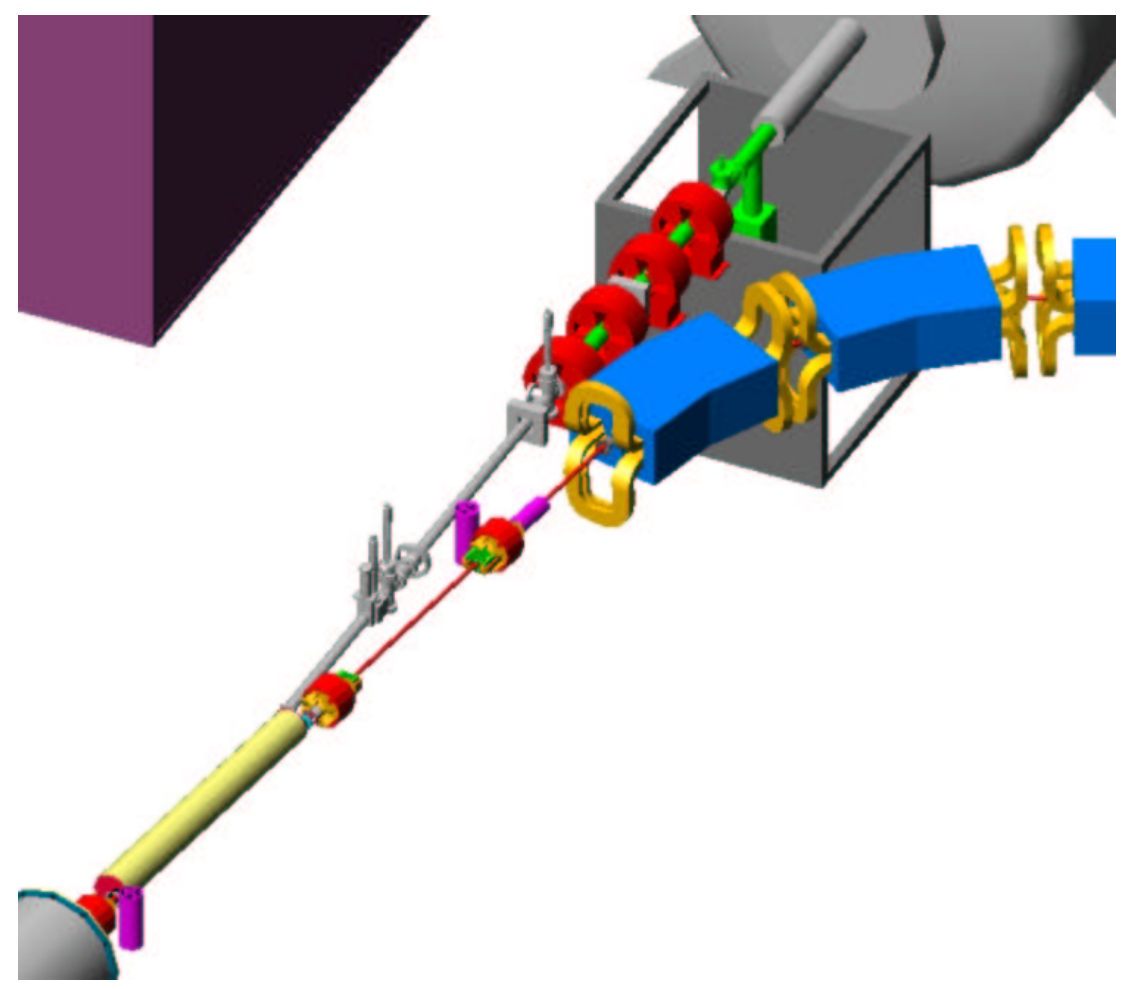

Figure 14.7: Perspective views of the injection interface.

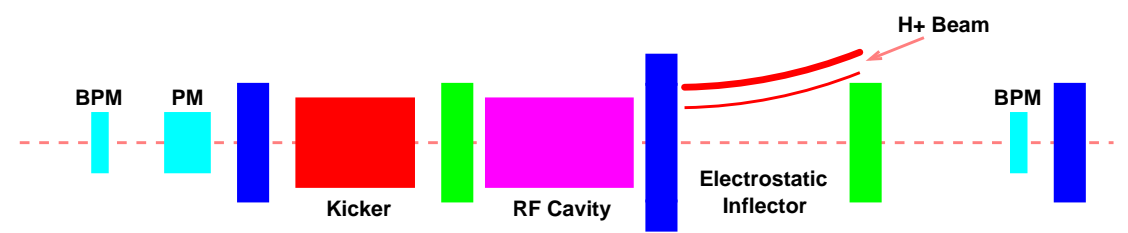

Figure 14.8: Schematic view of the interface between the tandem Van de Graaf injector and the synchrotron. 


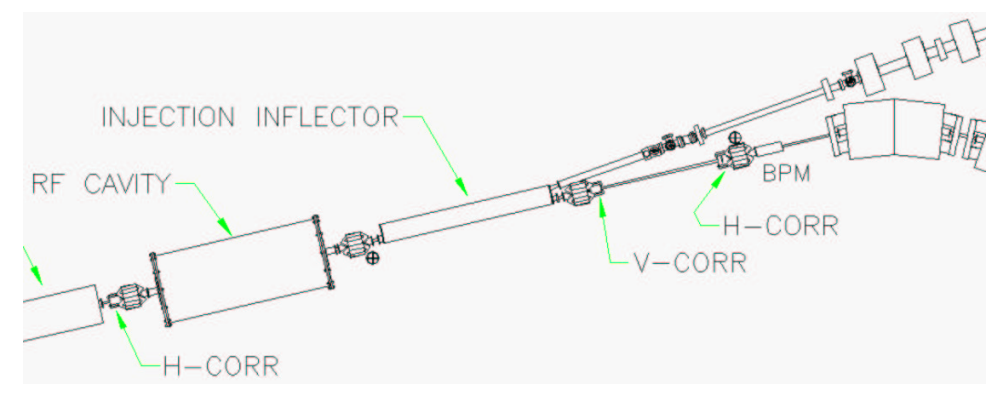

Figure 14.9: Plan view of the interface between the tandem Van de Graaf injector and the synchrotron.

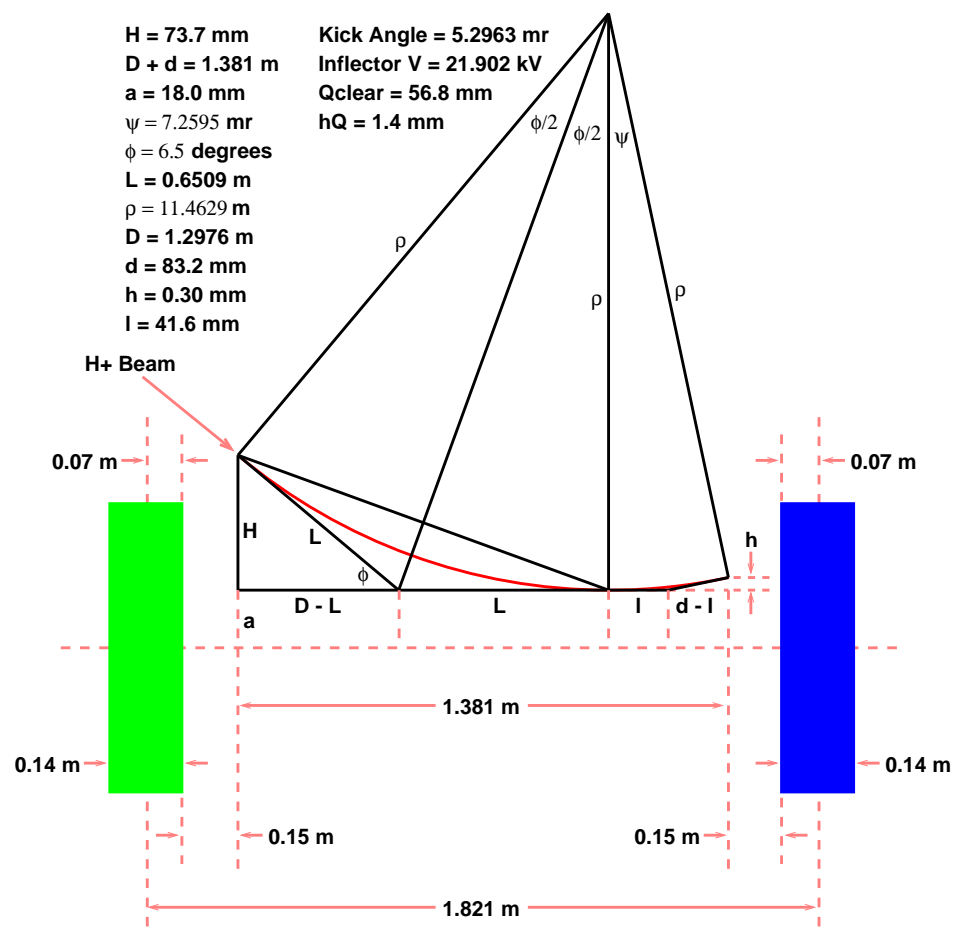

Figure 14.10: Nominal trajectory of beam passing through the injection inflector. (Note that beam enters from the left, in this sketch.) 


\section{Electrostatic Inflector}

$\begin{array}{lcc}\text { Bend angle, } \phi & & 6.5^{\circ} \\ \text { Radius of curvature, } \rho & {[\mathrm{m}]} & 11.5 \\ \text { Active length, } D+d & {[\mathrm{~m}]} & 1.4 \\ \text { Septum thickness } & {[\mathrm{mm}]} & 1 \\ \text { Gap, } g_{I} & {[\mathrm{~mm}]} & 18 \\ \text { Voltage, } V & {[\mathrm{kV}]} & 22 \\ \text { Electric field } & {[\mathrm{kV} / \mathrm{cm}]} & 12\end{array}$

\section{Injection Kicker}

Kick angle, $\Phi_{K}$

Magnetic length

Magnetic field, $B$

Gap, $g_{K}$

Current, NI

Rise time

Flat top

[mrad] 5.3

Fall time

(Revolution Period

[m] $\quad 0.2$

[G] $\quad 100$

[mm] 30

[A] 240

$[\mathrm{ms}]<16$

$[\mathrm{ns}]>100$

$[\mathrm{ns}]<600$

[ns] 840)

Table 14.3: Injection Kicker and Inflector Parameters 
gap of $g_{I}=18 \mathrm{~mm}$ between the septum and the cathode in the inflector. Thus the required electrostatic voltage is

$$
V=\frac{g_{I}}{\rho}\left(\frac{c^{2} p^{2}}{E}\right)=21.9 \mathrm{kV}
$$

Blue and green ellipses in Fig. 14.13 represent $\pm 2.5 \sigma$ (rms) of the beam at focusing and defocusing locations in the straights during injection, according to the values recorded in Table 4 of reference [55]. The comfortable physical aperture at injection becomes even more luxurious as the beam shrinks in transverse size during acceleration.

The injected beam leaving the inflector is on a trajectory that crosses the center line of the beam pipe at location $K$, in the middle of an injection kicker. This kicker is turned on to deliver a vertical magnetic field when the incoming beam passes for the first time, but is turned off on all subsequent beam passages. Thus it delivers a one-time horizontal kick to the beam, steering it to travel down the center of the beam pipe. The angle of the horizontal kick is

$$
\Phi_{K}=\frac{X_{I}}{\sqrt{\beta_{I} \beta_{K}} \sin \mu}=5.30 \mathrm{mr}
$$

where $X_{I}=18.3 \mathrm{~mm}$ is the incoming beam displacement at location $I$, the downstream end of the inflector, $\mu$ is the betatron phase advance between $I$ and $K$, and $\beta_{I}$ and $\beta_{K}$ are the horizontal beta functions at the two points. The integrated strength of the kicker is

$$
B l=\Phi_{K} B \rho=0.00203 T-m .
$$


The magnetic field required to do this in a $0.2 \mathrm{~m}$ long kicker is $B=101$ Gauss, which is delivered by a current $I$ such that

$$
N I=g_{K} B / \mu_{0}=242 \mathrm{~A}
$$

where $N$ is the number of turns wound on the kicker, and $g_{K}=30 \mathrm{~mm}$ is the magnet gap.

\subsection{Extraction}

Fig. 14.14 shows a general perspective of both injection and extraction interfaces, which are similar in many ways. The extraction kicker begins the extraction process by quickly turning on a vertical magnetic field during a selected turn number, thereby selecting the energy of the extracted beam. The angle is sufficient to move the beam horizontally across a current sheet at the upstream end of the extraction septum magnet, which also bends the beam horizontally. The positions of the extraction kicker and the extraction septum are shown schematically and in plan view in Fig. 14.15 and Fig. 14.16

Key parameters of the extraction kicker and the septum magnet are summarized in Table 14.4, for the nominal trajectory shown in Fig. 14.17. The required displacement of the beam at $S$, the entrance to the septum, is

$$
X_{S}=\Phi_{K} \sqrt{\beta_{K} \beta_{S}} \sin \mu=19.3 \mathrm{~mm}
$$

where $\mu$ is the phase advance from the center of the kicker, and $\beta_{K}$ and $\beta_{S}$ are

the horizontal beta functions. Solving this equation shows that the required 


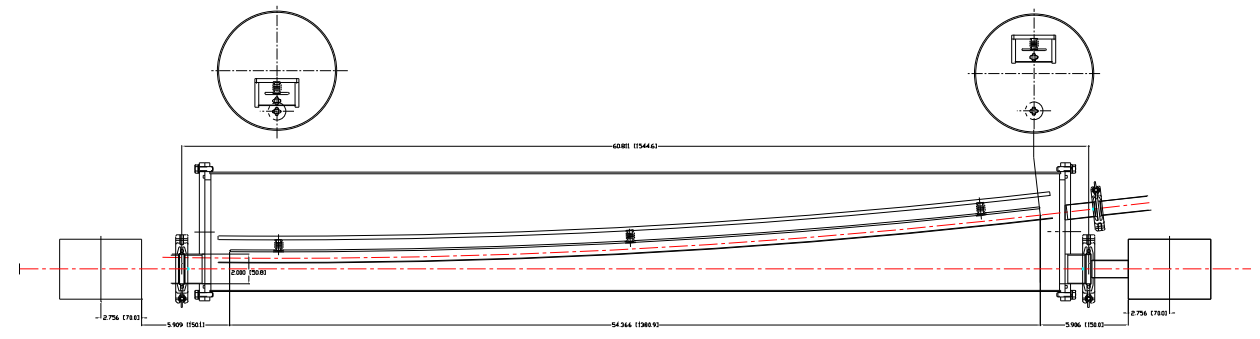

Figure 14.11: Plan view of the electrostatic injection inflector. Beam enters at the right from the tandem Van de Graaf injector.

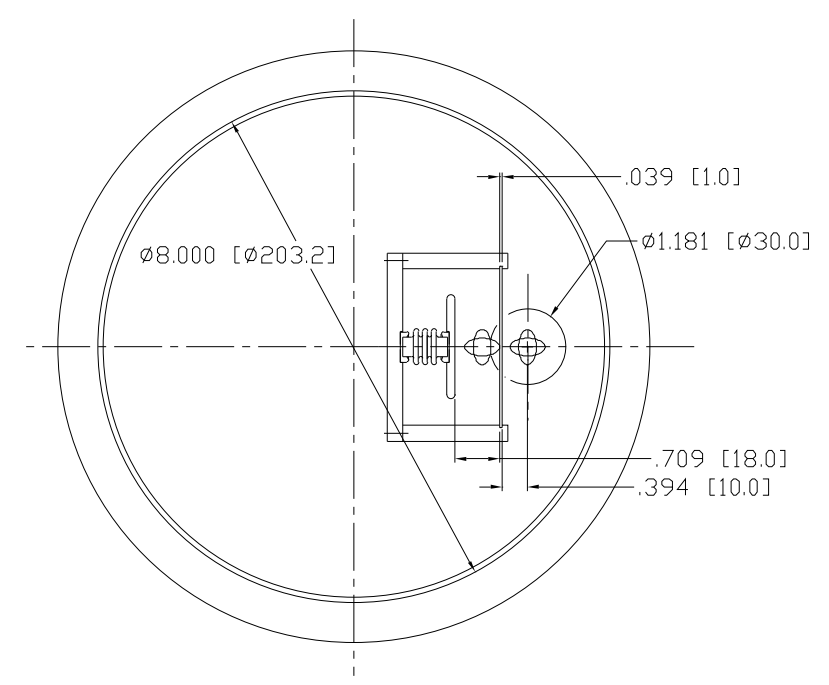

Figure 14.12: Apertures at downstream end of electrostatic inflector.

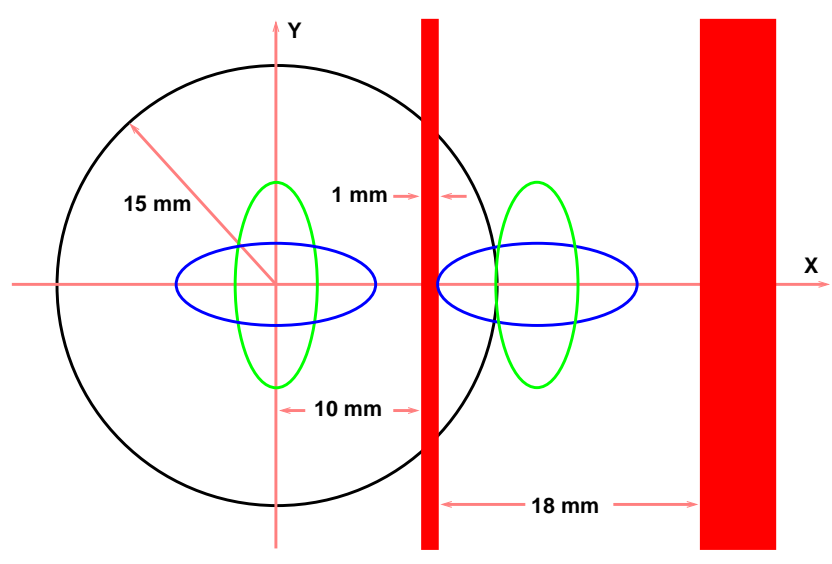

Figure 14.13: The green and blue ellipses in the schematic represent $\pm 2.5 \sigma$ (rms) of the beam envelope at the 1rostream and downstream ends of the inflector. 


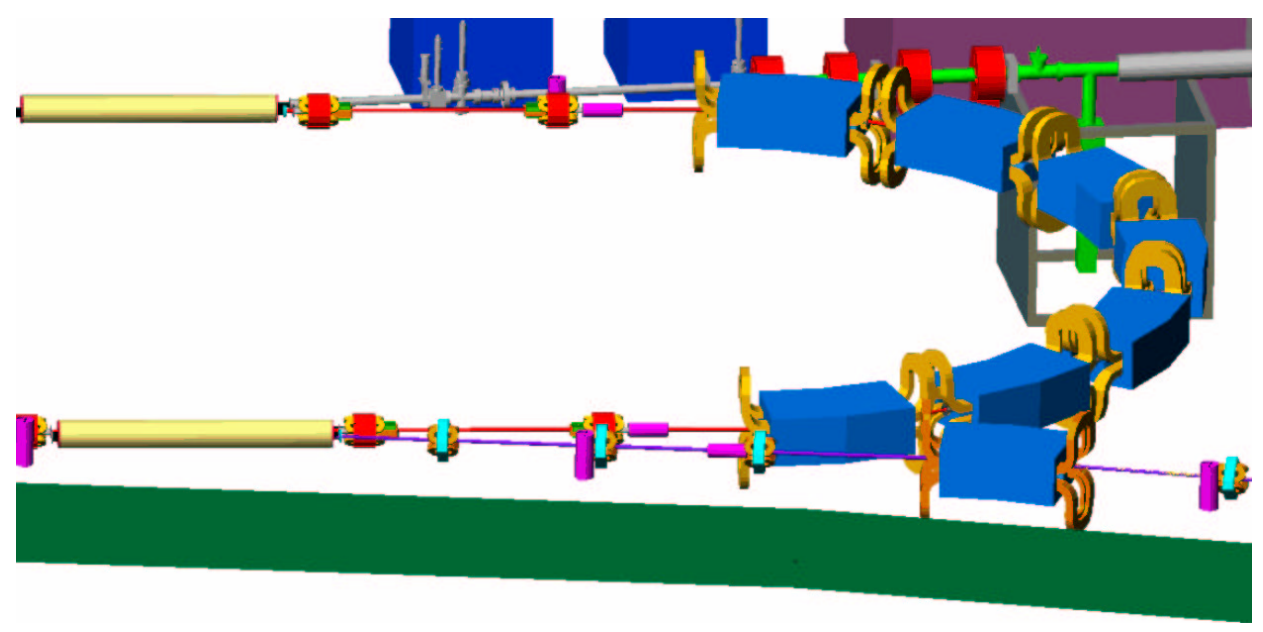

Figure 14.14: Perspective view of extraction (foreground) and injection (background) interfaces.

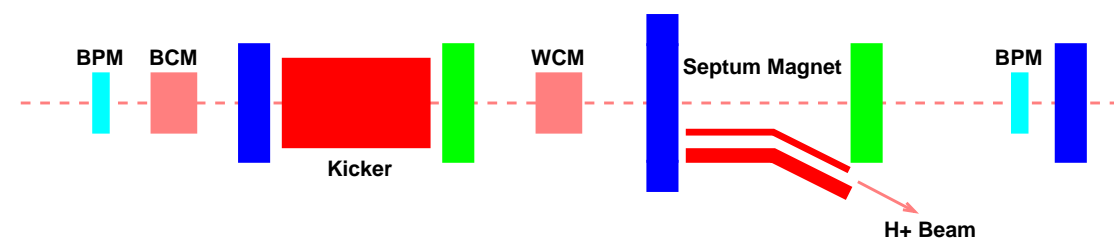

Figure 14.15: Schematic view of the extraction interface layout.

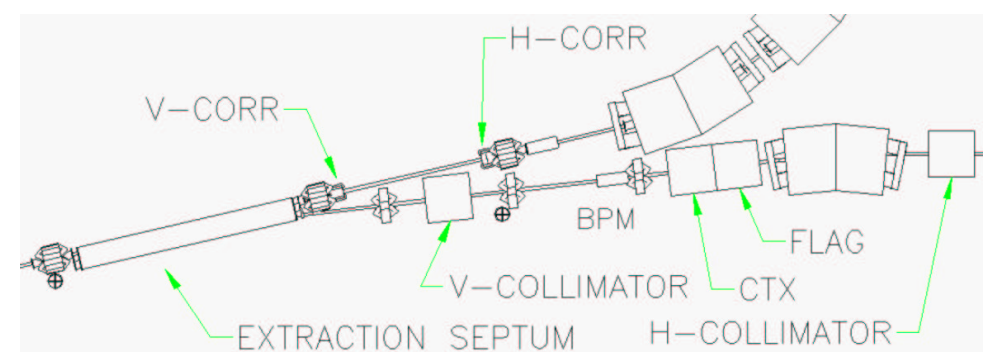

Figure 14.16: Plan view of the extraction interface layout. 


\begin{tabular}{|lcc|}
\hline Extraction Kicker & & \\
Bend Angle & {$[\mathrm{mrad}]$} & 5.48 \\
Magnetic strength & {$[\mathrm{Gm}]$} & 133 \\
Magnetic length & {$[\mathrm{m}]$} & 0.8 \\
Magnetic field & {$[\mathrm{G}]$} & 167 \\
Gap & {$[\mathrm{mm}]$} & 30 \\
Current & {$[\mathrm{A}]$} & 398 \\
Rise time & {$[\mathrm{ns}]$} & $<100$ \\
Flat top & {$[\mathrm{ns}]$} & $>70$ \\
Fall time & {$[\mathrm{ms}]$} & $<16$ \\
(Revolution Period & {$[\mathrm{ns}]$} & $167)$ \\
& & \\
Septum Magnet & & \\
Bend angle & & $6.5^{\circ}$ \\
Radius of curvature & {$[\mathrm{m}]$} & 12.268 \\
Length & {$[\mathrm{m}]$} & 1.481 \\
Magnetic field & {$[\mathrm{G}]$} & 1983 \\
Gap & {$[\mathrm{mm}]$} & 12 \\
Septum (Cu) thickness & {$[\mathrm{mm}]$} & 4 \\
Current & {$[\mathrm{A}]$} & 1893 \\
Half-sine pulse length & {$[\mu \mathrm{s}]$} & 10 \\
Ripple & & $<2 \%$ \\
& & \\
\hline
\end{tabular}

Table 14.4: Extraction Kicker and Septum Magnet Parameters 


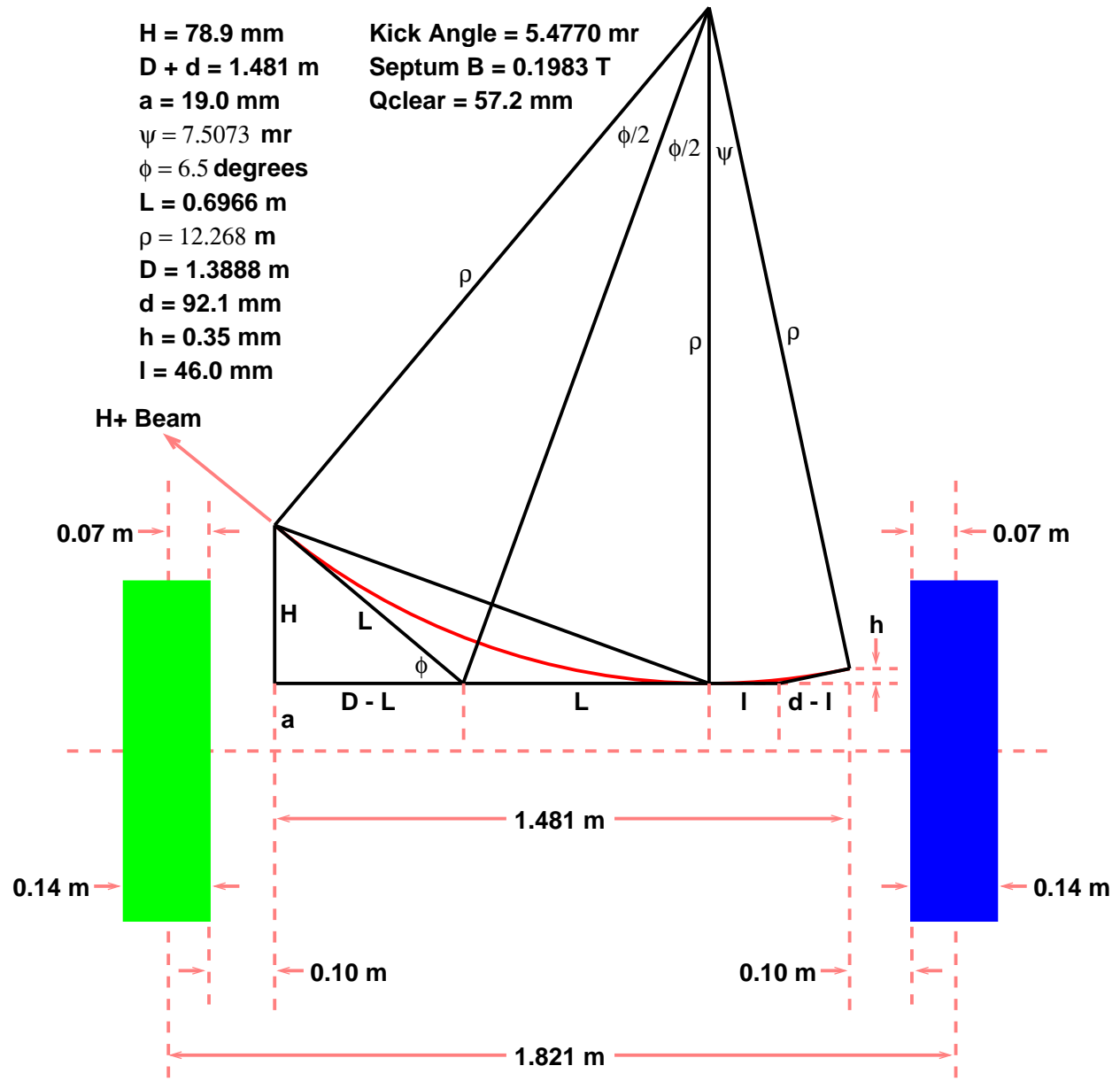

Figure 14.17: Nominal extraction trajectory of the beam leaving the synchrotron. 


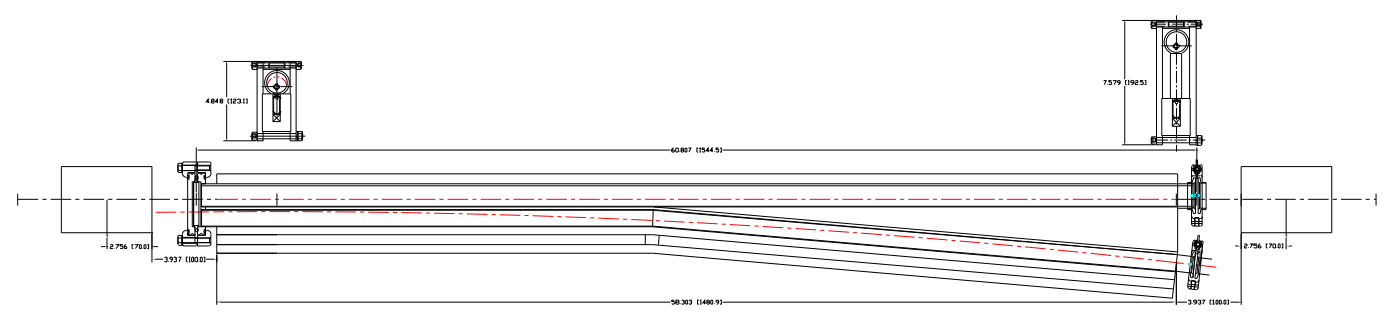

Figure 14.18: Extraction Septum Magnet

extraction kick angle is $\Phi_{K}=5.48 \mathrm{mr}$, requiring an integrated kick field of $0.0133 \mathrm{Tm}$. For a $0.8 \mathrm{~m}$ long kicker the required field is $B=167$ Gauss. This is delivered by a magnet with $N I=398$ Ampere-turns, if the gap height is $g=30 \mathrm{~mm}$, since

$$
B=\mu_{0} N I / g
$$

The current required in the septum magnet shown in Fig. 14.18 is also given by Equation 14.12, only now $B=0.1983 \mathrm{~T}$ and the gap is taken to be $g=0.012 \mathrm{~m}$, so that $N I=1893$ Amps. The apertures at the upstream end of the septum magnet are shown in Fig. 14.19 and the ellipses representing $\pm 2.5 \sigma$ for the beam at its largest, for the minimum extraction energy of $60 \mathrm{MeV}$ are shown in Fig. 14.20.

\subsection{Delivery System Optics}

The delivery system is composed of the extraction beam line, the switch yard beam line, the transport beam line, gantry optical interface, and gantries. The beam is extracted from the synchrotron by a fast kicker followed by a septum magnet. The extraction line comes just after the septum magnet. Since 


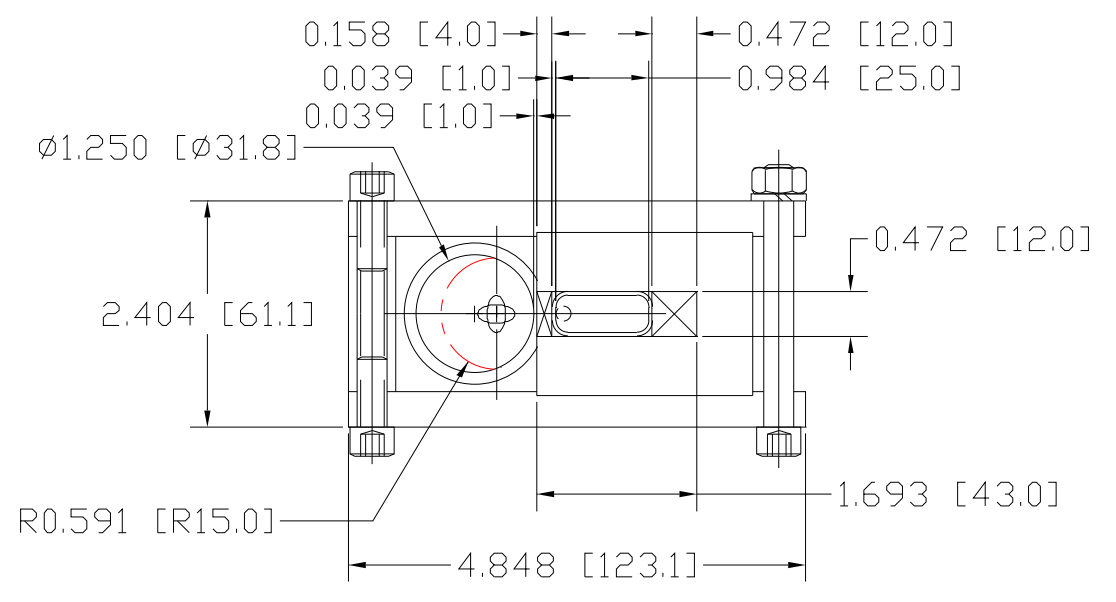

Figure 14.19: Plan view of the upstream end of the septum magnet, and septum magnet apertures.

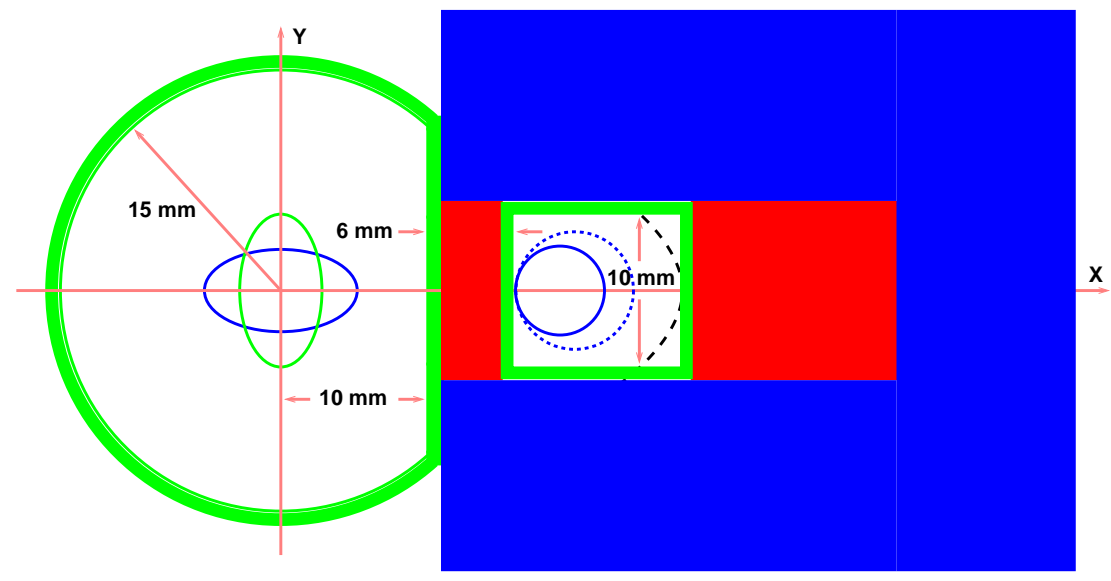

Figure 14.20: Schematic of the upstream end of the septum magnet, and septum magnet apertures. 
the switch yard is a configuration of FODO cells, IBEFUMFO can be directly applied in this case to make the optical matching between the extraction line and the switch yard beam line. The extraction line is designed such that the output lattice functions comply with equation 13.16 and also the dispersion is zero outside the extraction line (see Fig. 14.21). Since the gantries all have an identical design it is desirable to have the same lattice functions at the entrance of these beam lines. The switch yard has been designed with this objective in mind as a perfect periodic structure.

The transport lines take the beam from the switch yard to the different rooms of the facility. The research room has two transport lines with bending angles that differ by 30 degrees. The fix beam room has one 45 degrees transport line that goes to the vertical fixed beam line and two additional 90 degrees transport line. The transport lines that connect the switychyard with the gantry are all identical and the same as the 45 degree transport lines used in the the fix beam room.

The gantry requires axially symmetric optics at the point of rotation. A special optical structure that we have named "gantry optical interface" has been designed to achieve this objective by making the horizontal and vertical beta function equal at the point of rotation and the alpha functions equal to zero (see Fig 14.22). The matching is done by inserting 3 quadrupoles between the transport line and the gantry. The distances between the quadrupoles and the strengths of two of them are varied until the matching conditions are satisfied 


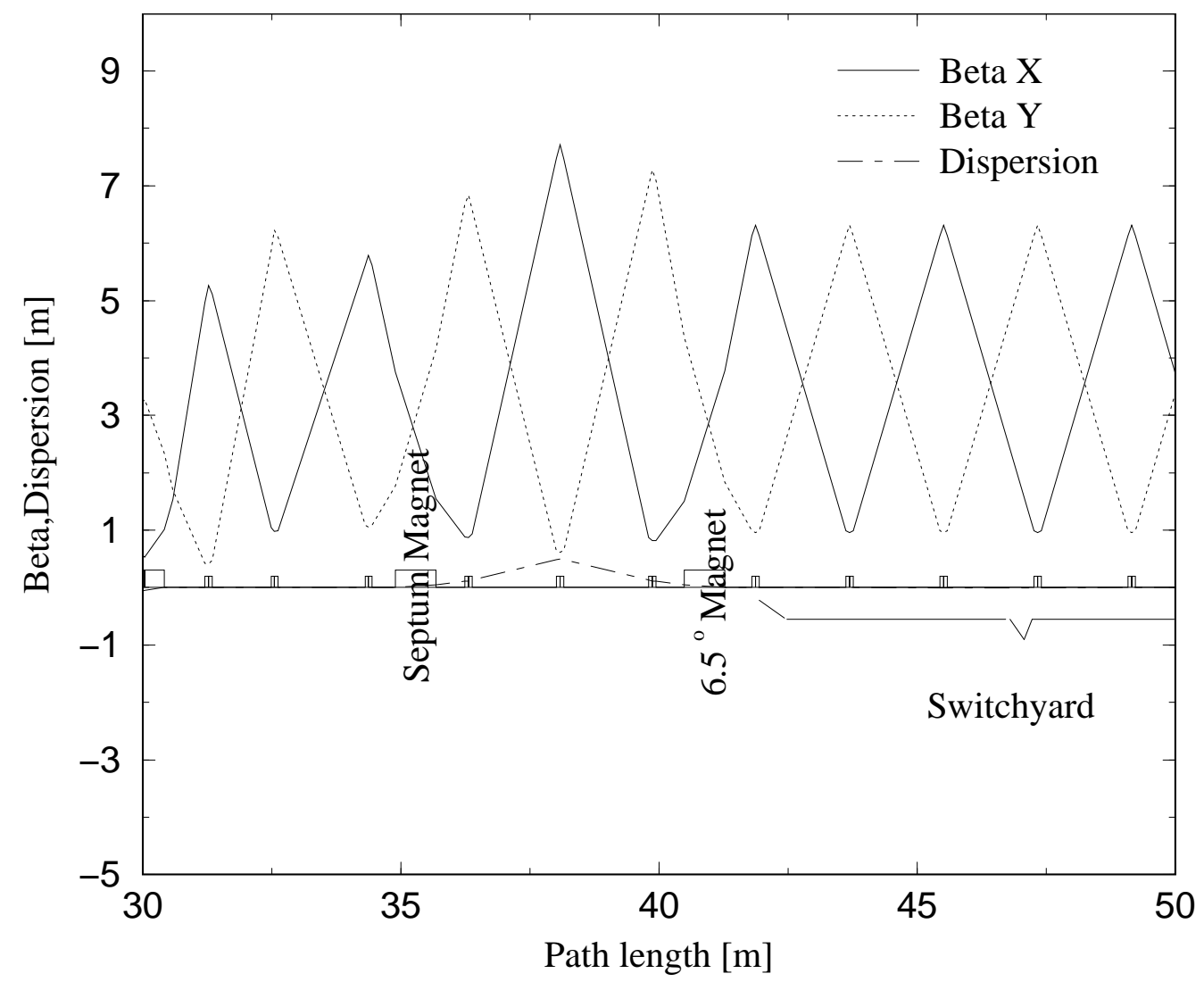

Figure 14.21: The interface between the extraction line and the switch yard simultaneously suppress the dispersion initiated by the septum magnet and smoothly matches the beta functions to the FODO cells structure of the switch yard. 


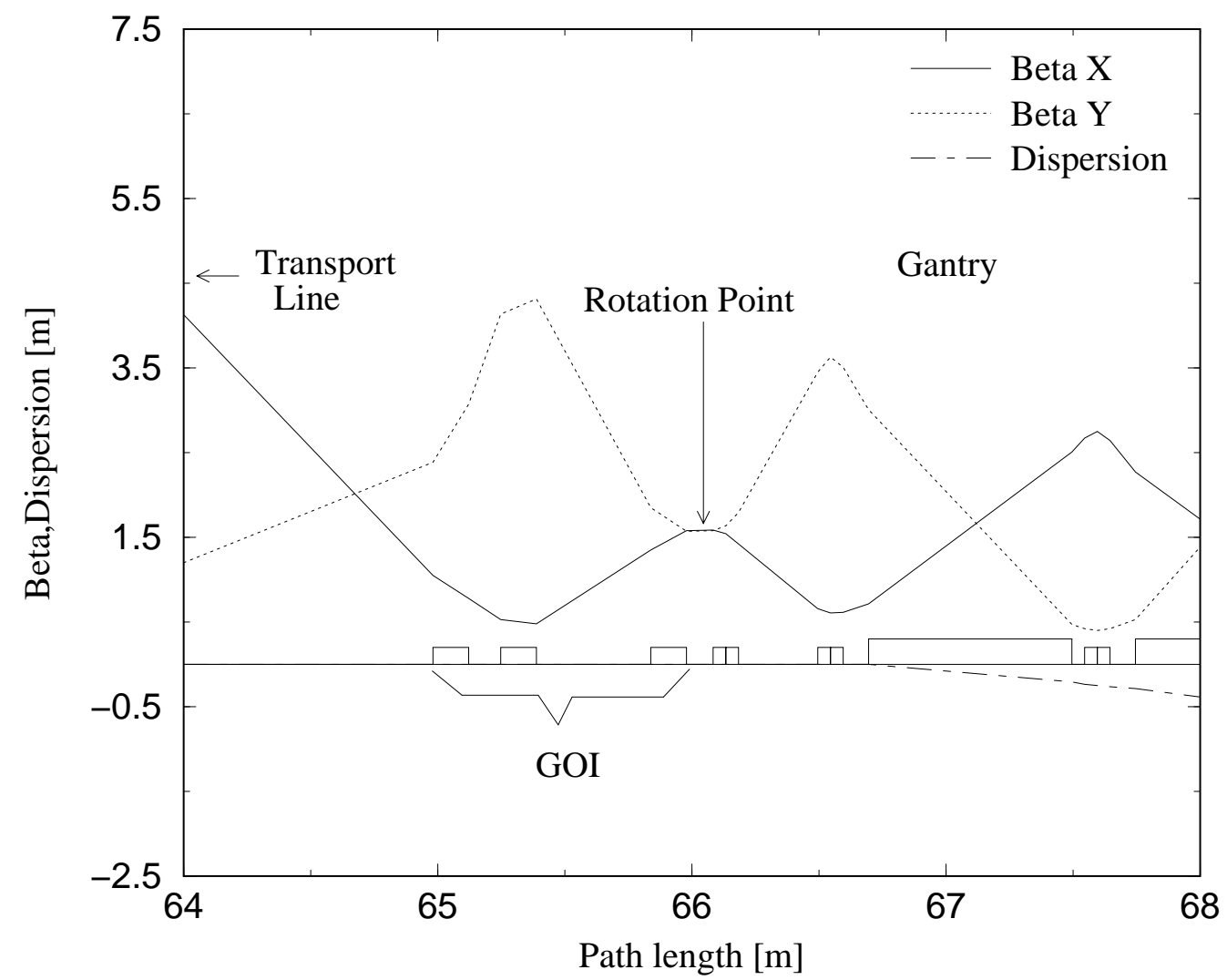

Figure 14.22: The distances and strengths of 3 quadrupoles before the rotation point are set such that the alpha functions are equal to zero and the beta functions are equal in both planes. 


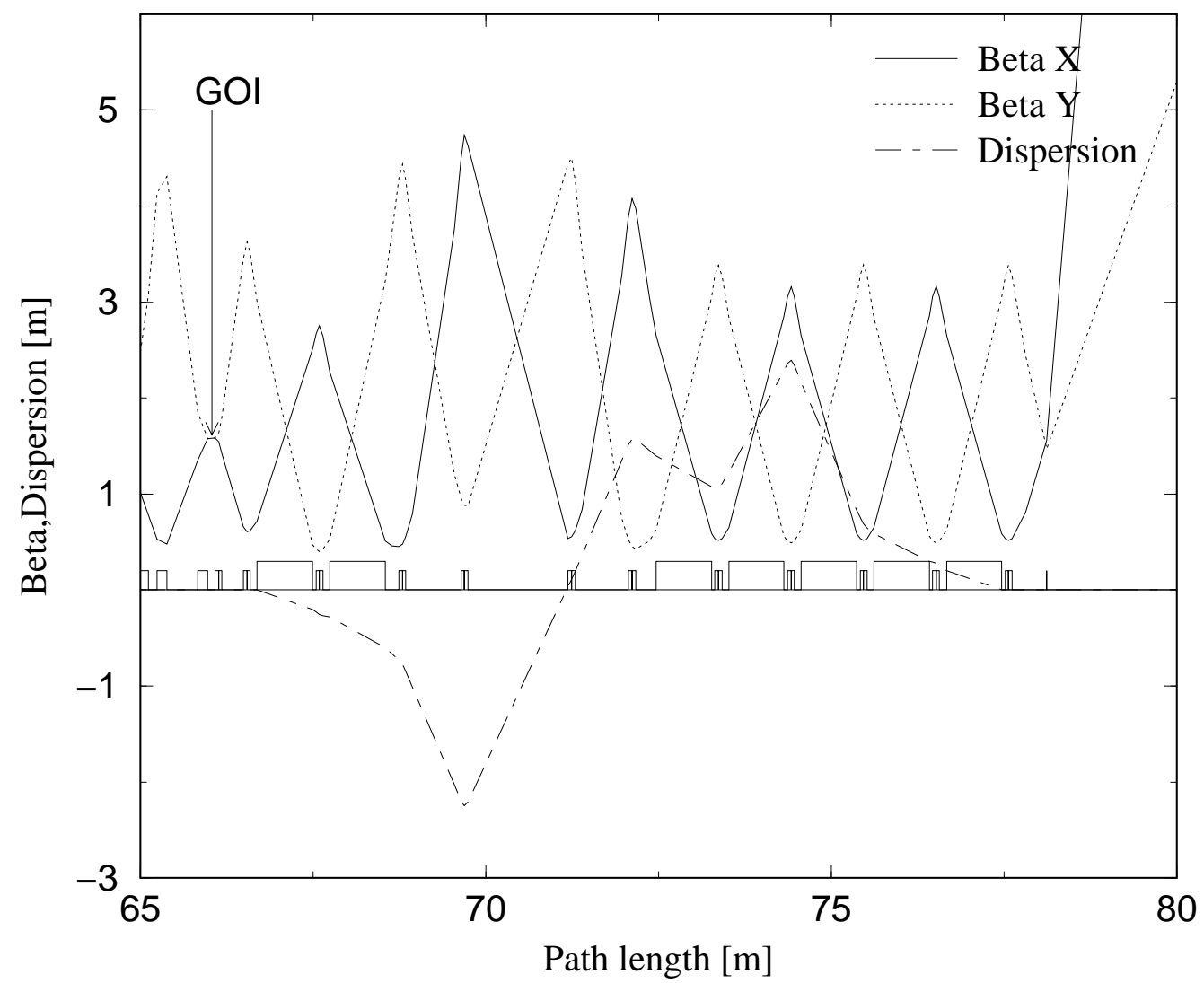

Figure 14.23: The achromatic gantry is built with seven 30 degrees dipole. Dispersion is match such that it is zero at the gantry output.

For the design of the gantry light weight and compactness is of highest priority. The gantry dipole deflects the beam by 30 degrees instead of 22.5 degrees of the magnets used in the switchyard and the transport line, maximizing the "packing factor" (ratio of integrated dipole length to the total length). Seven 30 degrees dipoles are used to built the gantry and only 4 quadrupole power supplies. Lattice functions of the gantry can be seen in Fig. 14.23. 


\begin{tabular}{|l|r|r|}
\hline & Nominal & Max. Variation \\
\hline $\mathrm{Qx}$ & 3.37 & -0.063 \\
$\mathrm{Qy}$ & 3.39 & -0.080 \\
$\max \beta_{x}(m)$ & 5.79 & 0.265 \\
$\max \beta_{y}(m)$ & 6.23 & 0.002 \\
$\max \eta_{x}$ & 2.01 & 0.034 \\
\hline
\end{tabular}

Table 14.5: A variation of the quadrupole strengths by $2 \%$ lead to the changes shown on the table. The variations are considered insignificant for operations purposes.

\subsubsection{Tolerances of the New Ring}

It is neccesary to test this lattice against any possible change of the quadrupole strength that might distort significantly the lattice functions, the dispersion or the tunes. In order to do that, the quadrupoles strengths in the arcs were varied about $2 \%$ from their nominal values. The maximum variation of tunes, dispersion and beta functions are summarized on Table 14.5. The variations shown on Table 14.5 are considered insignificant for operation purposes.

\subsection{Magnet Considerations}

The RCMS originally was designed to have 6 different dipole magnets styles (see Table 14.6): a Chevron magnet used in the synchrotron ring, a Ctype magnet used in the switch yard and transport lines, an H-type rectangular magnet used in the gantry, an $\mathrm{H}$-type rectangular magnet used as a $6.5^{\circ}$ magnet in the extraction line, and two O-type rectangle magnet for the horizontal and 
vertical correctors respectively. There are also 2 different styles of quadrupoles, one for the synchrotron and other for the gantry.

The Chevron magnet is a special kind of dipole built out 2 rectangular bends. This procedure reduces the maximum excursion of the beam (beam sagita) within the beam pipe and hence it is possible to use smaller beam pipes [42].

In order to reduce costs, trajectory studies were done to determine the feasibility of reducing the number of dipole styles. In particular, the possibility of using the old synchrotron magnets as a switch yard magnets (C-type) is studied in Fig 14.24. This Fig. shows the beam pipe and the beam itself when the magnet is off (straight beam pipe) and the corresponding pictures when the magnet is on (curved beam pipe) over the magnet iron. The pipes fit exactly within the iron of the synchrotron dipole allowing this dipole to be used as switch yard dipole also.

Similar studies have shown that it is also possible to use a 22.5 degrees synchrotron dipole as the 6.5 degrees dipole (see Fig. 14.25) needed in the extraction line.

The new synchrotron dipole as mentioned before, is a combined function magnet with a bending angle of $25.71^{\circ}$ and the same magnet is also a chevron magnet as in the old version.

In summary, the number of style of magnets have been reduced from 6 to 5 (see Table 14.6) which will have a direct impact on the cost of the facility not only in the design stage but also during commissioning and normal operation. 


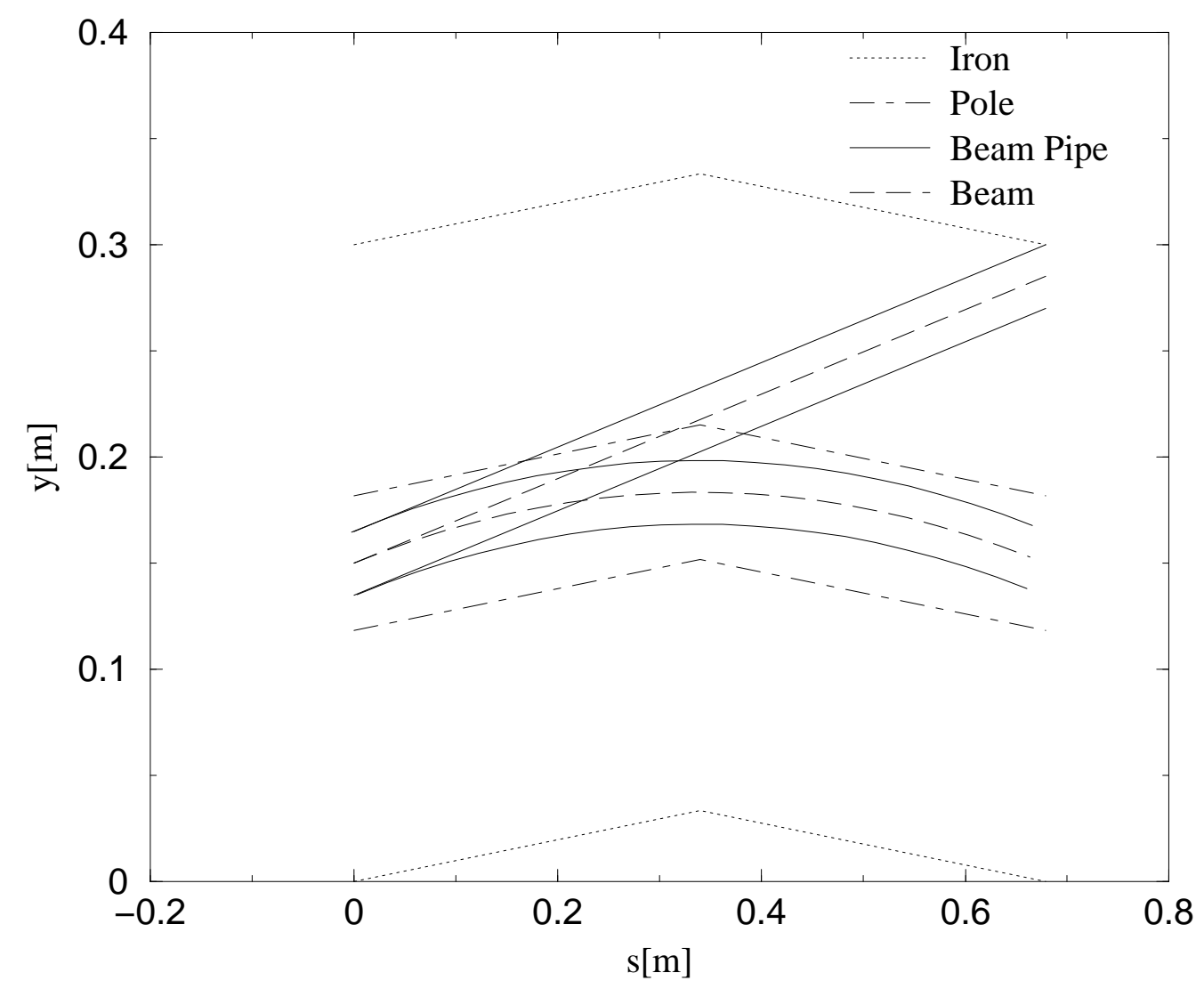

Figure 14.24: The trajectories and beam pipes of the switch yard magnet within the iron of the 22.5 degrees dipole showing that the beam can go through the magnet even when it is off. 


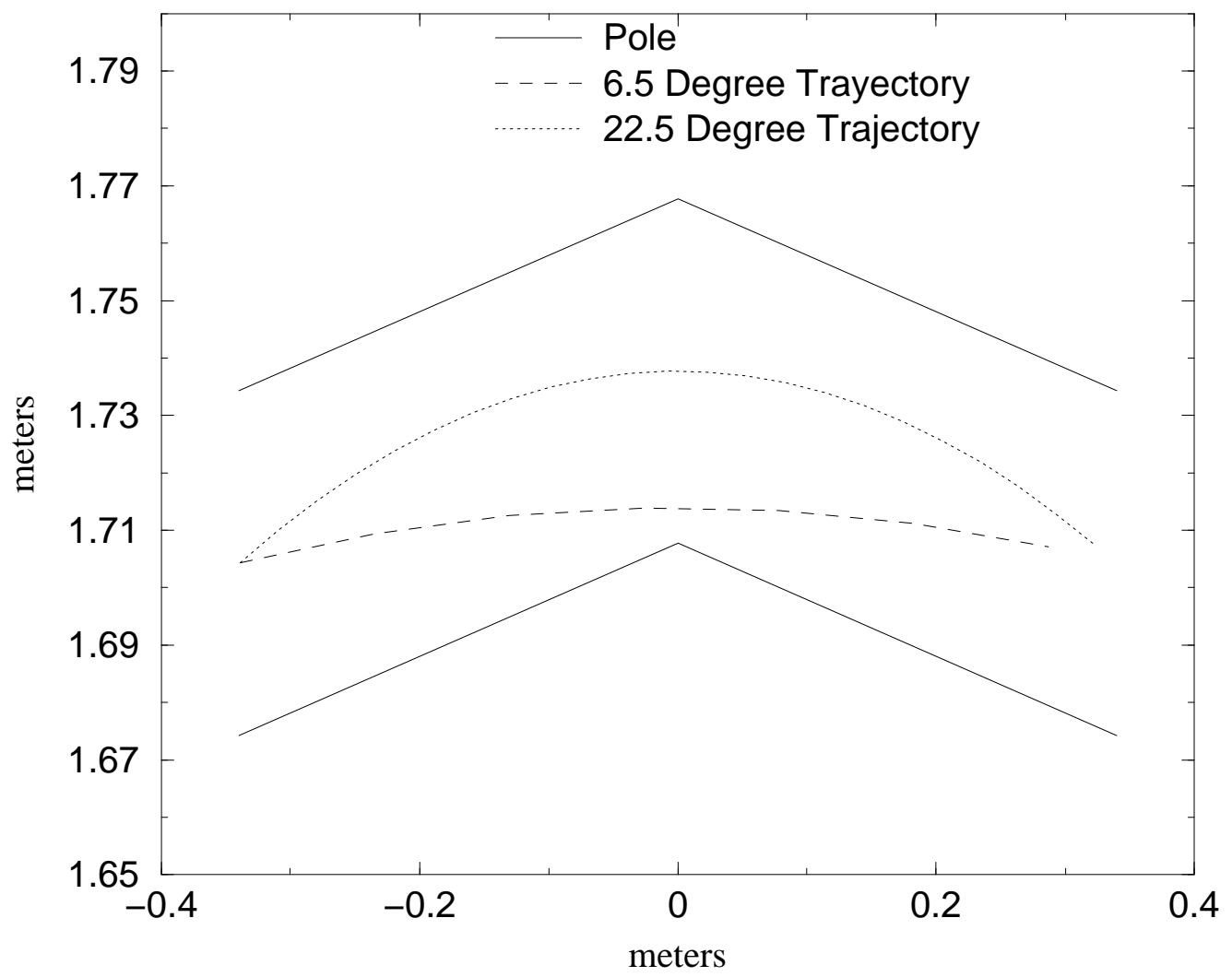

Figure 14.25: The trajectory of the beam when the $22.5^{\circ}$ magnet is used as $6.5^{\circ}$ magnet. The $6.5^{\circ}$ trajectory fits loosely within the pipe of the magnet. 


\begin{tabular}{|l|c|c|}
\hline Magnet Style & No (Before) & No (After) \\
\hline $25.71^{\circ}$ Chevrons & 0 & 14 \\
$22.5^{\circ}$ Chevrons & 16 & 21 \\
H-type (gantry) & 35 & 35 \\
H-type (6.5 $)$ & 1 & 0 \\
C-type & 22 & 0 \\
O-type (Hor) & 4 & 4 \\
O-type (Ver) & 4 & 4 \\
\hline
\end{tabular}

Table 14.6: Comparison between the number of magnets needed before and after doing the trajectory studies. Those studies show that C-type magnets and 6.5 degree magnet can be replaced with combined function magnets.

\begin{tabular}{|l|r|}
\hline System & Cost Change \\
\hline Magnets & $-22.6 \%$ \\
Inj. \& Extr. & $-10.0 \%$ \\
Power Supplies & $-6.5 \%$ \\
Radio Frequency Cavities & $-50.0 \%$ \\
\hline
\end{tabular}

Table 14.7: Cost change between some of the systems of the old design of the RCMS and the corresponding ones to the most recent design.

\subsection{Comparisons with Previous Designs}

The previous versions of the RCMS used separated magnets for the dipoles and quadrupoles in the arcs of the ring. The introduction of combined function magnets in the new design has saved sixteen quadrupoles.

The switch yard was also redesigned with the aid of the IBEFUMFO procedure leading to a perfectly periodic structure with a reduced number of quadrupole. The total number of quadrupoles in the delivery system went down from 175 quadrupoles to 139 quadrupoles. 
Taking into account the above considerations, the total cost of the design and fabrication of all magnets has dropped by $22.6 \%$ as can be seen on Table 14.7 .

Since the total number of magnets has been reduced, the requirements on the power supplies are less and hence it has been possible to lower their fabrication cost by $6.5 \%$ as shown on Table 14.7.

The RF system in the previous design were composed of two one gap cavities powered by solid state amplifiers. Since the straight sections now have a longer space available $(1.8 \mathrm{~m})$ it was possible to design a single cavity of two gaps to provide the neccesary energy to the particles each turn. This modification on the RF system then lower the fabrication cost of the cavities by $50 \%$ as indicated on Table 14.7 .

The injection and extraction system have also benefited from the longer straight sections. Since the electrostatic inflector used at injection and the septum magnet used at extraction are now longer the voltage required for the inflector and the magnetic field required for the septum magnet have each been reduced by about one third. This reduces the cost of these systems in the amount listed in Table 14.7.

\subsection{Comparisons with other Medical Accel- erators}

Reference [41] makes a comparison of 4 different technologies for medical accelerators. Although the comparisons are based in parameters beyond 
Table 14.8: Magnet Pole Gap and Magnet Pole Tip Width for 3 Different Facilities.

\begin{tabular}{|l|c|c|}
\hline Facility & $\begin{array}{c}\text { Magnet Pole Gap } \\
{[\mathrm{mm}]}\end{array}$ & $\begin{array}{c}\text { Magnet pole tip width } \\
{[\mathrm{mm}]}\end{array}$ \\
\hline Loma Linda [45] & 50 & 200 \\
KUMPF [48] & 67 & 340 \\
RCMS [56] & 30 & 60 \\
\hline
\end{tabular}

the optical design is worth to mention some of the results. First, it is concluded that the RCMS can achieve faster changes in the beam energy than any other accelerator with a much smaller emittance, a moderate size an adequate intensity for proton therapy.

Second, the energy of the beam can be easily varied in the required range of energies by just firing the extraction kicker at different times. In contrast, in the cyclotron the beam is extracted at maximum energy and reduced to the desired energy by a variable thickness energy degrader. In this sense, the delivered energy selection is variable, at the expense of a higher emittance from multiple scattering, larger gantries, and a higher radio activation which leads to higher shielding requirement.

From the optical point of view, the RCMS have a very small beam size when compared to typical medical facilities already in operation like Loma Linda or facilities in design stage like KUMPF (Kyoto University Medical Proton Facility) (see Table 14.8) and since the aperture size is directly correlated with the magnet size the RCMS has smaller magnets when compared with the other facilities. For example, the transverse section of the dipole magnets 
that is used in the KUMPF is $97 \mathrm{X} 80 \mathrm{~cm}$ [48] while the transverse section of the dipole magnets in the RCMS is only $48 \mathrm{X} 24 \mathrm{~cm}$. Since the RCMS magnets are smaller than the ones employed in other facilities, the overall cost of the RCMS optical lattice is also expected to be lower.

\subsection{Conclusions}

The overall optical lattice of the RCMS has been presented making special emphasis in the IBEFUMFO algorithm employed to facilitate the design. In particular, IBEFUMFO has made possible to increase the spacing of the quadrupoles in the straight sections without increasing the beam size in the arcs and with a modest increase of the beam size in the straight sections. The increase of the spacing of the quadrupoles in the straight sections allows to use small angles of extraction and also allow more space for the RF cavity which reduces the cost of both the extraction system and the RF cavity.

IBEFUMFO has also been used in the design of the delivery optics, in particular in the extraction line where the algorithm has been used to match the incoming beta function of the ring to the beta functions of switch-yard, a beam line made out of FODO cells and which feeds all the beam lines going to the different rooms in the facility.

Particle trajectory studies inside the different magnet styles indicate that it is possible to use the $22.5^{\circ}$ Chevron magnet as a C-type magnet and as a 6.5 degree magnet. This reduces the number of magnets styles from 6 styles 
to 5 styles.

Comparisons of this design with previous designs show significant savings not only in the RF and extraction system as was mentioned before but also in the number of magnets to be employed in the facility.

Comparisons with other medical facilities has shown technological advantages of the RCMS and most likely lower costs thank to the smaller magnets of the RCMS. 


\section{Chapter 15}

\section{Effect of the Eddy Current in the beam of the RCMS}

\subsection{Introduction}

The RCMS uses a relatively high repetition rate $(30 \mathrm{hz})$ which raises concerns about the eddy currents induced in the vacuum chamber and eventually the effects that this Eddy current might have in the stability of the beam. This Chapter evaluates several effects that the Eddy currents usually produce like tune shifts and reduction of the dynamical apertures.

\subsection{Magnetic Multipoles Generated by Eddy Currents}

The magnetic multipoles generated by Eddy currents have been studied in detail in reference [50]. Calculation in this reference starts under the assumption that an Eddy current can be seen as an infinite long filament of

current $I$ inside two parallel iron plates with infinite permeability and separa- 
tion distance $g$. Application of the image current method allows to calculate the magnetic field $\left(H=H_{y}+i H_{x}\right)$ at position $z=x+i y$ produced by the filament located at $z_{c}=x_{c}+i y_{c}$ as:

$$
H=\frac{I}{4 g}\left[\tanh \frac{\pi\left(z-z_{c}^{*}\right)}{2 g}+\operatorname{coth} \frac{\pi\left(z-z_{c}\right)}{2 g}\right] .
$$

After Taylor expanding the previous equation we have:

$$
B=\frac{\mu_{0} I}{4 g} \sum_{n=0}^{\infty} \frac{1}{n !}\left(\alpha_{n}+\beta_{n}\right)\left(\frac{\pi}{2 g}\right)^{2} z^{n}
$$

where:

$$
\begin{aligned}
& \alpha_{n}=\left.\frac{\partial^{n} \tanh \left(z-\frac{\pi z_{c}^{*}}{2 g}\right)}{\partial z^{n}}\right|_{z=0} \\
& \beta_{n}=\left.\frac{\partial^{n} \cosh \left(z-\frac{\pi z_{c}^{*}}{2 g}\right)}{\partial z^{n}}\right|_{z=0}
\end{aligned}
$$

The current in terms of the magnetic field that originates such current is:

$$
I=\int_{v . c .} J d A=\int_{v . c .} \sigma \dot{B} x h d s
$$

where $J$ is the current density, $\sigma$ is the electrical conductivity of the chamber walls, $\dot{B}$ is the rate of change of the field, $x$ is the distance measured from the chamber center and $h$ is the thickness of the chamber walls.

Now since the magnetic field can be written in function of its multipoles components $a_{n}$ and $b_{n}$ as:

$$
B(z)=B_{0}\left(1+\sum_{n=0}^{\infty}\left(b_{n}+i a_{n}\right) z^{n}\right)
$$




\begin{tabular}{|c|c|c|c|}
\hline $\mathrm{a}[\mathrm{cm}]$ & 2 & 4 & 6 \\
\hline$b_{2}\left[1 / \mathrm{m}^{2}\right]$ & 0.160 & 0.502 & 0.670 \\
\hline
\end{tabular}

Table 15.1: Sextupole components induced by Eddy currents in an elliptical beam pipe for different values of the major semi-axes $a$

Eq. 15.2, Eq. 15.7 and Eq. 15.6 lead to:

$$
b_{n}+i a_{n}=\frac{\mu_{0} \sigma h \dot{B_{0}}}{2 \pi n ! B_{0}}\left(\frac{\pi}{2 g}\right)^{n+1} \int_{v . c .} x\left(\alpha_{n}+\beta_{n}\right) d s
$$

The variables $\alpha_{n}$ and $\beta_{n}$ can be calculated analytically and for the sextupole case they are given by:

$$
\begin{aligned}
& \alpha_{2}=-2 \frac{\tanh \left(-\frac{\pi z_{c}^{*}}{2 g}\right)}{\left(\cosh \left(-\frac{\pi z_{c}}{2 g}\right)\right)^{2}} \\
& \beta_{2}=2 \frac{\operatorname{coth}\left(-\frac{\pi z_{c}^{*}}{2 g}\right)}{\left(\sinh \left(-\frac{\pi z_{c}}{2 g}\right)\right)^{2}}
\end{aligned}
$$

The sextupoles components can now be found from Eq. 15.8 by numerical integration. Values of the $b_{2}$ has been estimated in reference [50] for different beam pipe shapes and sizes. In particular, the values of $b_{2}$ for an elliptical beam pipe of different major semi-axes have been tabulated on Table 2 of such reference and only the sextupole component of such table has been reproduced here for convenience on Table 15.1. The sextupoles depends on more parameters besides $a$ and they are:

$$
\begin{aligned}
h & =1 \mathrm{~mm}, \\
\sigma^{-1} & =0.8 \mu \Omega m,
\end{aligned}
$$




$$
\dot{B} / B=30 / s .
$$

Since these parameters are either directly proportional or inversely proportional to the sextupole strength (see Eq. 15.8) it is straightforward to scale the values given on Table 15.1 to other different set of parameters.

The sextupole strength also depends on the minor axis of the ellipse $b$ through the integral of Eq. 15.8. According to reference [50] such dependence is approximately inversely proportional.

Table 15.1 and the known relations of the different parameters allow to quickly estimate the sextupole strength for the RCMS which has parameters:

$$
\begin{aligned}
a & =3 \mathrm{~cm} \\
b & =1.5 \mathrm{~cm} \\
h & =0.64 \mathrm{~mm}, \\
\sigma^{-1} & =1.25 \mu \Omega m, \\
\dot{B} / B & =188.49 / \mathrm{s},
\end{aligned}
$$

leading to a sextupole strength of $0.687 \frac{\mathrm{T}}{\mathrm{m}^{2}}$ or an integrated sextupole strength of $0.522 \frac{T}{m}$ in each main dipole of the ring. 


\subsection{Marylie Simulations}

In order to evaluate the effect of the sextupole components in the beam , particularly dynamic aperture a tune footprints, particles were tracked with the software Marylie ([51]). Marylie uses a "map" that translate the initial phase space coordinates into the corresponding phase space coordinates at the end of the ring. Simulation of the particles going through many turns are done by successively applying the mentioned maps.

Maps used by Marylie are built with the so called Lie transformations ( see for example [52],[53]) . The Lie transformations are defined as:

$$
e^{: f:} \equiv \sum_{m=0}^{\infty} \frac{: f:^{m}}{m !},
$$

where the Lie operator $: f:$ is defined as:

$$
: f: \equiv \sum_{i}\left(\partial f / \partial q_{i}\right)\left(\partial / \partial p_{i}\right)-\left(\partial f / \partial p_{i}\right)\left(\partial / \partial q_{i}\right)
$$

Any map can be approximated by:

$$
\mathcal{M}=e^{: f_{2}:} e^{: f_{3}:} e^{: f 4:} \ldots
$$

where $f_{n}$ is a homogeneous polynomial of degree $\mathrm{n}$ in the phase space variables.

The importance of the Lie transformations resides on the concept of simplecticity. Orbits of charged particles are well described by Hamiltonian flows. As a consequence any map used to translate phase space coordinates from one place of the ring to another should comply with the so called symplectic 
condition. This condition can be mathematically express by:

$$
M^{t}\left(z^{i n}\right) J M\left(z^{i n}\right)=J \quad \text { for all } z^{i n}
$$

where $J$ is given by:

$$
J=\left(\begin{array}{cc}
0 & I \\
-I & 0
\end{array}\right)
$$

and $I$ is the $3 \mathrm{X} 3$ identity matrix. Any Lie transformation is symplectic and the product of Lie transformations is also symplectic therefore the maps built out of Lie transformations are automatically symplectic. If this property and Eq. 15.14 are combined one can conclude that a map built out of Lie transformations can be approximated to any order and the map is still symplectic.

This is no the case for Taylor expansions of the map in which a truncation of the series unavoidable leads to a loss of simplecticity of the map since coefficients of different orders of the expansion are dependent of each other through the simplecticity condition. Since usually the particles has to be tracked for large numbers of turns this loss of simplecticity even in a very small amounts might lead to unphysical results.

\subsubsection{Preparation of the Lattice and Main Dipole Split- ting}

The design of the lattice was done in the simulation program MAD [10]. It is then necessary to convert the different elements used in MAD to their corresponding elements in the Marylie software. There are some few details about this conversion. First the quadrupoles strengths in MAD were defined 


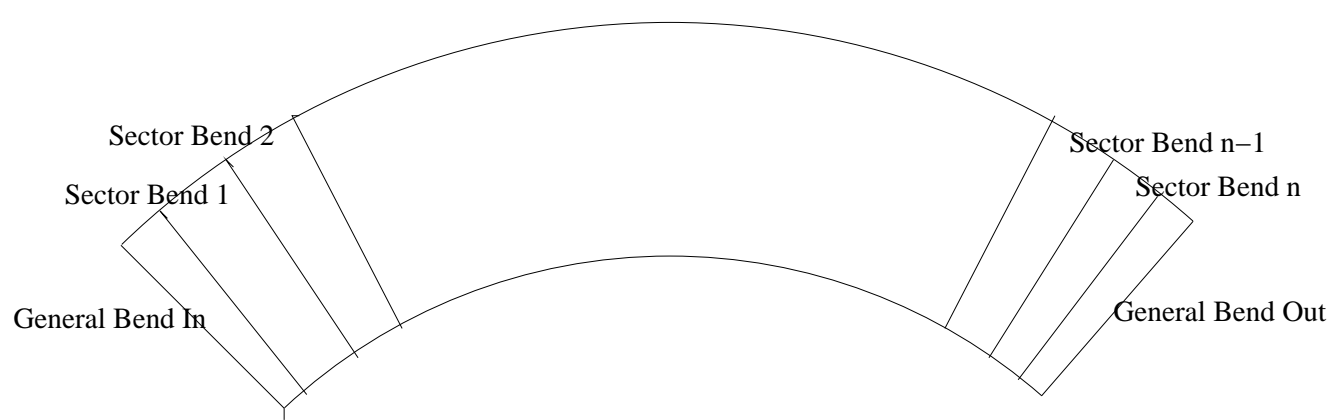

Figure 15.1: The main dipoles are divided in $\mathrm{n}$ sector bends and the multipolar elements are inserted between them. The edges of the magnet are carefully modeled with general bending magnets that allow individual pole phase rotation of the faces of the magnet. Fringe fields in the edge are also added.

as the ratio between the real physical quadrupole strength and the rigidity of the particle. In Marylie, the quadrupole strengths correspond numerically to the physical quadrupole strengths. This means that the rigidity has to be specified in Marylie.

Second, dipoles in Marylie are specified with different parameters than the dipoles parameters used by MAD. In Marylie the dipoles are specified with a bend angle and a magnetic field while in MAD the dipoles are specified with a bend angle and a length.

Third, Marylie doesn't have built in elements that allow to put sextupole components in the dipoles. It is then, neccesary to divide the main dipoles and place thin multipoles at the divisions in order to simulate the effect of the sextupoles. The main body of the divided magnet will consist then of sector bends with multipoles located at every common point between the mentioned sector bends (see Fig. 15.1). 


\begin{tabular}{|l|c|c|}
\hline Parameter & $\begin{array}{c}\text { After Direct } \\
\text { Conversion }\end{array}$ & $\begin{array}{c}\text { Readjusted to get } \\
\text { MAD tunes }\end{array}$ \\
\hline Def. Quad. Strength & -0.0003344 & -0.0003317 \\
Foc. Quad. Strength & 0.0003329 & 0.0003320 \\
Tunex & 0.3909710 & 0.3905060 \\
Tuney & 0.4562570 & 0.4304580 \\
\hline
\end{tabular}

Table 15.2: Quadrupoles strengths of the multipoles inserted in the main dipoles used in the Marylie Software. Direct conversion from the MAD lattice leads to tunes that differ slightly from the design ones. Readjustment of the quadrupole strengths in the third significant figure are then neccesary to bring back the tunes to their original values.

Also quadrupole components have to be added to the main magnet since the dipole are combined function magnets as explained in Chapter 14. This is easily done with the inserted thin multipoles that support not only sextupole but also quadrupole components and higher order multipole components.

The number of divisions in the dipole is determined by how much the resultant map change when the number of divisions changes. Allowing a maximum change of $0.01 \%$ in the terms of the map, the number of division needed turn out to be 1984 .

Since the magnets has been designed with pole phase rotations special care has to be taken at the edge of the magnets. The edges are built with maps that represent magnets with pole phase rotation and maps that represent fringe fields in sector bends.

Once the dipoles have been appropriately divided and the whole lattice has been written in terms of Marylie elements, it is neccesary to make some comparisons with MAD outputs like tunes and lattice functions. 
Comparison between the tunes given by Marylie and Mad lead to small differences that start to be evident in the second significant figure (see Table 15.3.1). Since the tunes are one of the most determinant parameters in tracking simulations the quadrupole components of the dipole magnets were slightly re-tuned until it was possible to achieve an agreement between the MAD and Marylie tunes of at least 6 significant figures. The beta functions produced by both packages were also compared as illustrated in Fig. 15.2. The fractional differences between the output lattices produced for both packages reaches values as big as $1 \%$.

\subsubsection{Effect of the Sextupole Components on the Dy- namic Aperture of the RCMS Ring}

Before doing the tracking simulations neccesary to determine the dynamic aperture of the ring, it is convenient to determine the general effect of the sextupole in the phase space ellipses. Extensive tracking simulations were done in Marylie for this purpose. In particular, the final distribution of particles on an initial 4D 2-torus distribution for different sextupole strengths was studied (see Fig. 15.3 and Fig. 15.4). Fig. 15.3 and Fig. 15.4 show some growth of the phase space ellipses when the strength of the sextupole is increased. The horizontal phase space ellipses on Fig. 15.3 tend also to get distorted as the sextupole strength is increased. Negative values of the sextupole strengths were also studied leading to phase space ellipses thickness growth even smaller than the ones shown in Fig. 15.3 and Fig. 15.4. 


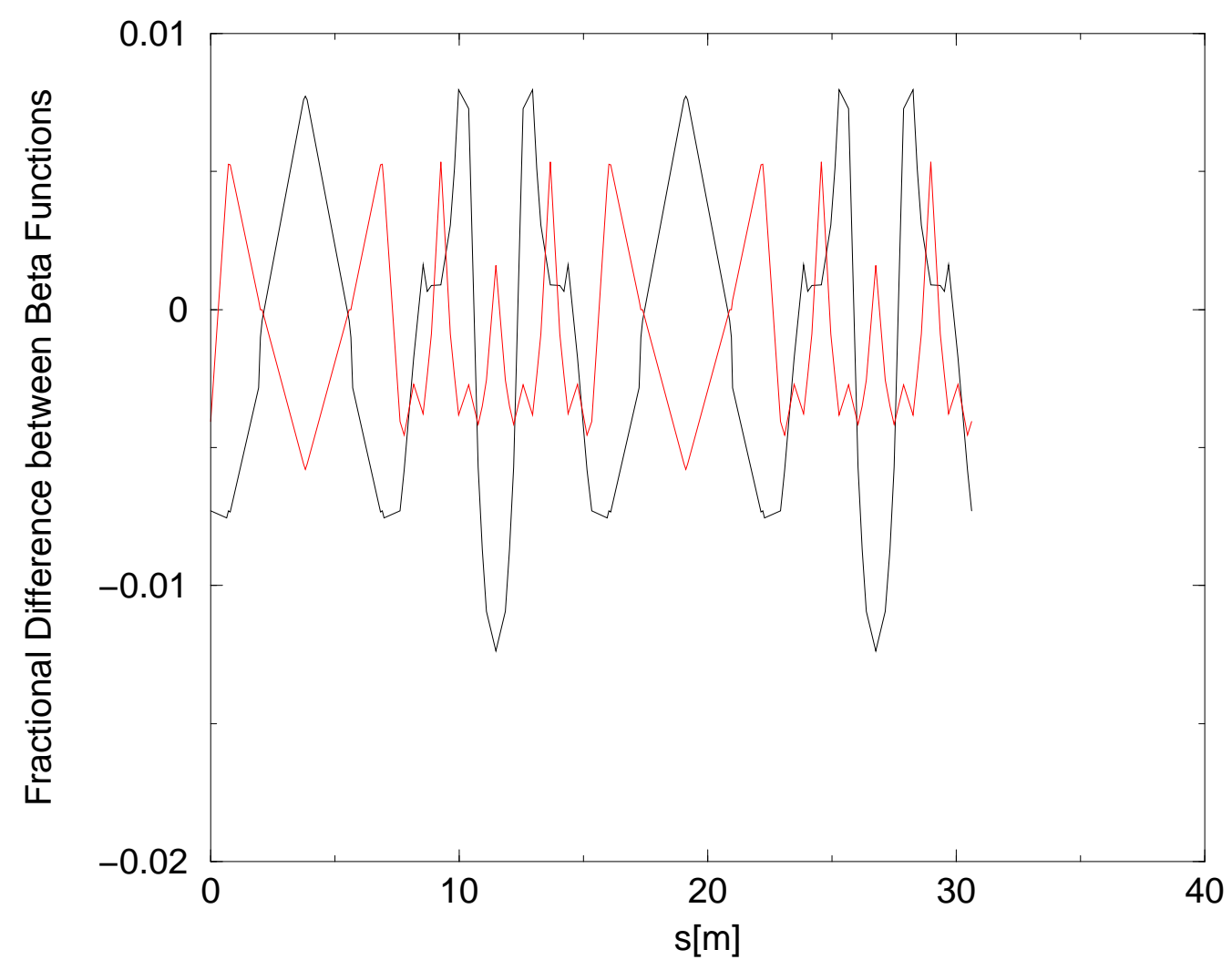

Figure 15.2: Computation of the fractional difference of beta functions between MAD and Marylie. The black line corresponds to the fractional difference of the horizontal beta functions while the red line corresponds to the fractional difference of the vertical beta functions. 


\section{Sextupole Effect on Phase Space Ellipses}

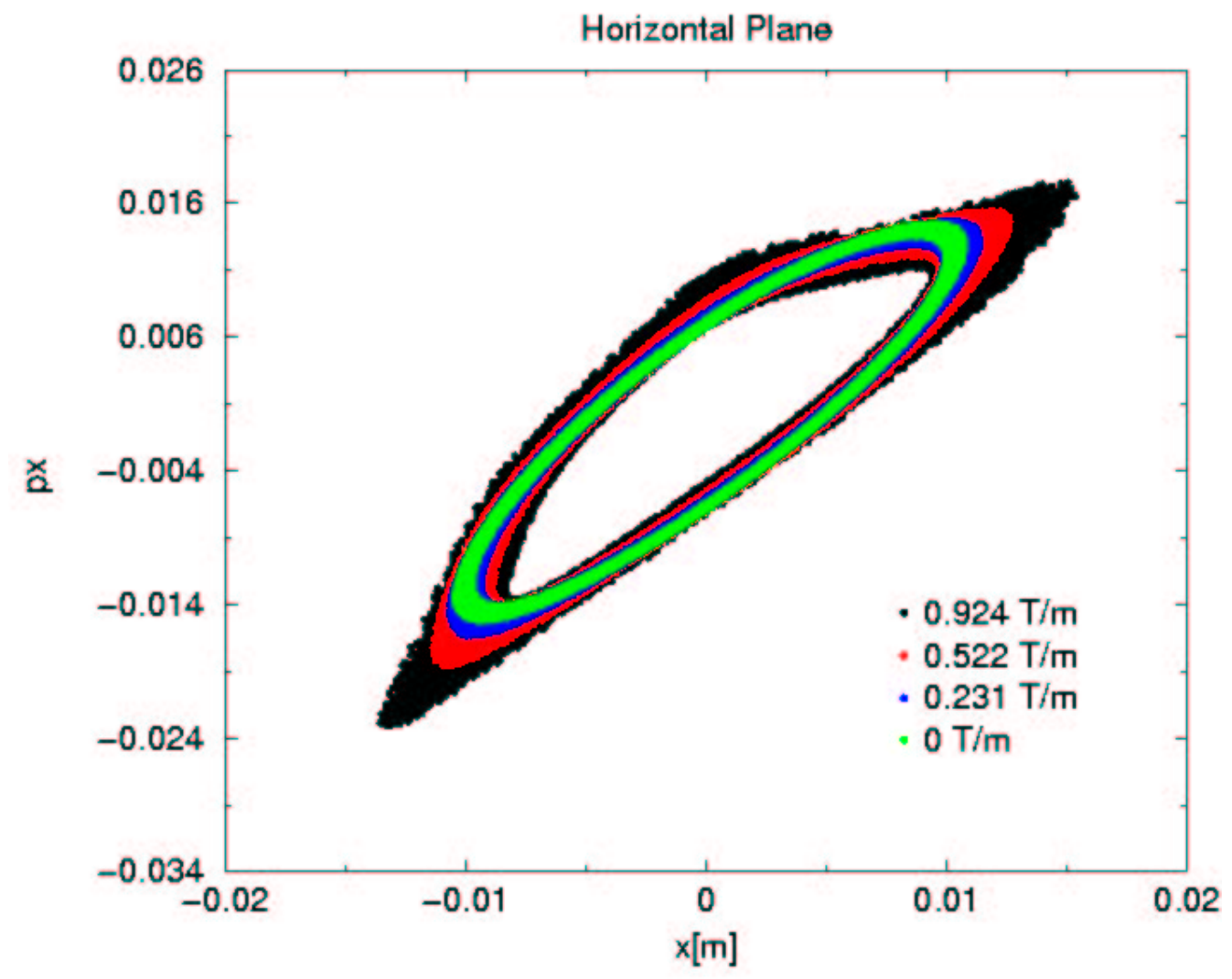

Figure 15.3: Horizontal phase space ellipses at one location of the ring after tracking for 62530 turns with different integrated sextupole strengths. The nominal sextupole strength of $0.522 \mathrm{~T} / \mathrm{m}$ is represented by the red ellipse. 


\section{Sextupole Effect on Phase Space Ellipses}

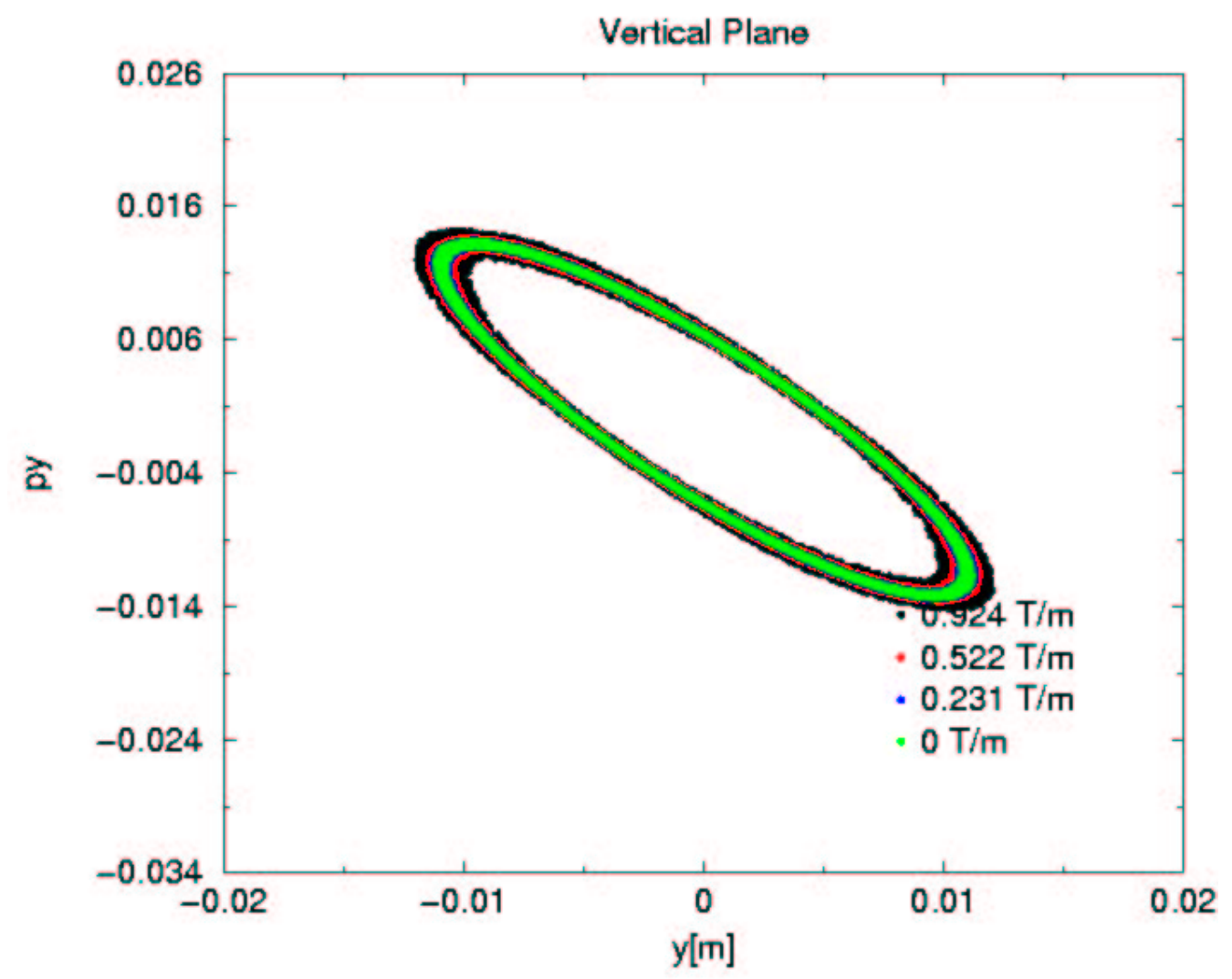

Figure 15.4: Horizontal phase space ellipses at one location of the ring after tracking for 62530 turns with different integrated sextupole strengths. The nominal sextupole strength of $0.522 \mathrm{~T} / \mathrm{m}$ is represented by the red ellipse. 


\begin{tabular}{|c|c|c|c|}
\hline $\begin{array}{c}\text { Sext. Str. } \\
{[\mathrm{T} / \mathrm{m}]}\end{array}$ & Qx' & Qy' & $\begin{array}{c}\mathrm{r} \\
{[\mathrm{mm}]}\end{array}$ \\
\hline-0.924 & 91.56 & -9.20 & 10.2 \\
-0.522 & 64.70 & 11.05 & 10.9 \\
-0.231 & 45.32 & 25.66 & 14.1 \\
0 & 29.90 & 37.28 & 10.9 \\
0.231 & 14.49 & 48.90 & 10.8 \\
0.522 & -4.89 & 63.51 & 11.2 \\
0.924 & -31.76 & 83.75 & 8.7 \\
\hline
\end{tabular}

Table 15.3: The radius $r$ for which particles start to get lost in the tracking simulations is found as function of the sextupole strength. The chromaticities $Q x^{\prime}$ and $Q y^{\prime}$ are also calculated in each run.

Having evaluated the effect of the sextupoles in the phase space ellipses the dynamic apertures of the ring can now be evaluated. This can be done by finding the biggest ellipse allowed in the ring before particles start to get lost. This is a process that involved tracking of particles several times in the ring, every time with a different size particle distribution.

The process was repeated for all the sextupoles strengths studied before (positive and negative) with results that are summarized on Table 15.3. The maximum beam size of the RCMS is expected to be as big as $3 \mathrm{~mm}$ at one sigma(see Chapter 14). Even in the worst case when the sextupole strength is +0.924 (which is almost double the nominal strength), the maximum transverse radius allowed for the particles before they start to get lost $(r=8.7 \mathrm{~mm})$ is just enough to keep most of the particles inside the beam pipe during the acceleration cycle of the RCMS. 


\subsubsection{Footprints}

Non linear components in the ring can introduce some dependence of the particles tune with the amplitude of oscillation. In the RCMS it is important to know if the tune spread caused by the eddy currents and other non linear effects can excite unwanted resonances in the ring.

In order to calculate such tune spread a uniform distribution of particles in the $J x$-Jy space (see Fig. 15.5) were tracked for 62530 turns with the Marylie software. The tunes of each individual particle were determined using the orbit information provided by the 62530 simulated turns. The particles are then sorted out according to their horizontal and vertical actions, $J_{x}$ and $J_{y}$, given place to Fig. 15.6 and Fig. 15.7. These two graphs are done with the nominal sextupole strength of $0.522 \mathrm{~T} / \mathrm{m}$ and a bigger strength of $0.924 \mathrm{~T} / \mathrm{m}$.

The biggest tune spread in the horizontal plane is about $8.15 e^{-4}$ and $2.55 e^{-4}$ in the vertical plane both of them for the extreme situation of a sextupole strenght of $0.924 \mathrm{~T} / \mathrm{m}$. These tune spreads are no large enough to move the nominal tune into the strongest resonances of one half or one third and hence the operation of the machine is stable within the sextupole range considered in this study.

\subsection{Conclusions}

The sextupole component induced by the eddy currents in the beam pipe has a visible effect in the phase space distribution of the particles but the 


\section{Initial Distrtibution of Particle}

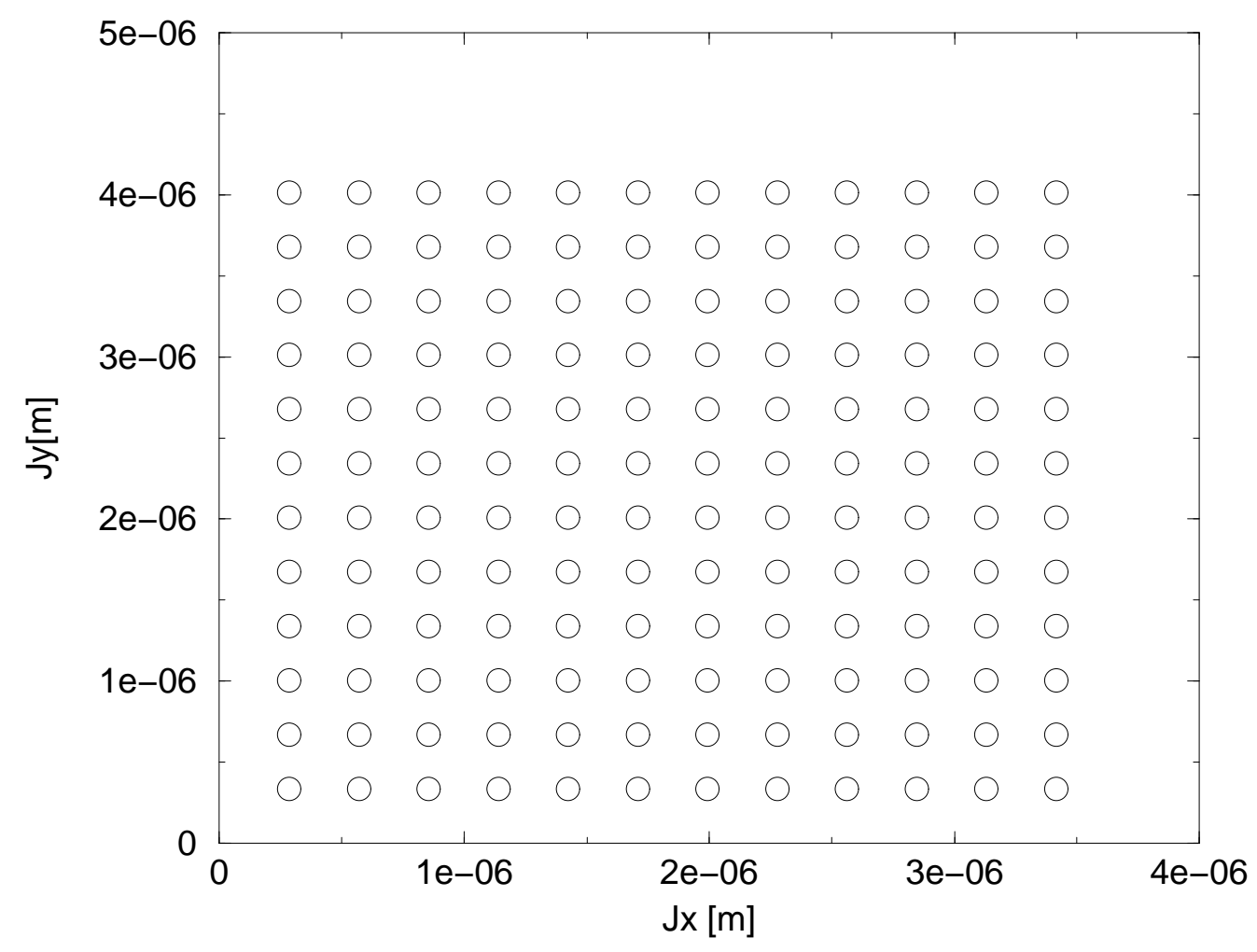

Figure 15.5: Amplitude distribution of particles before doing tracking to evaluate tune footprints. 


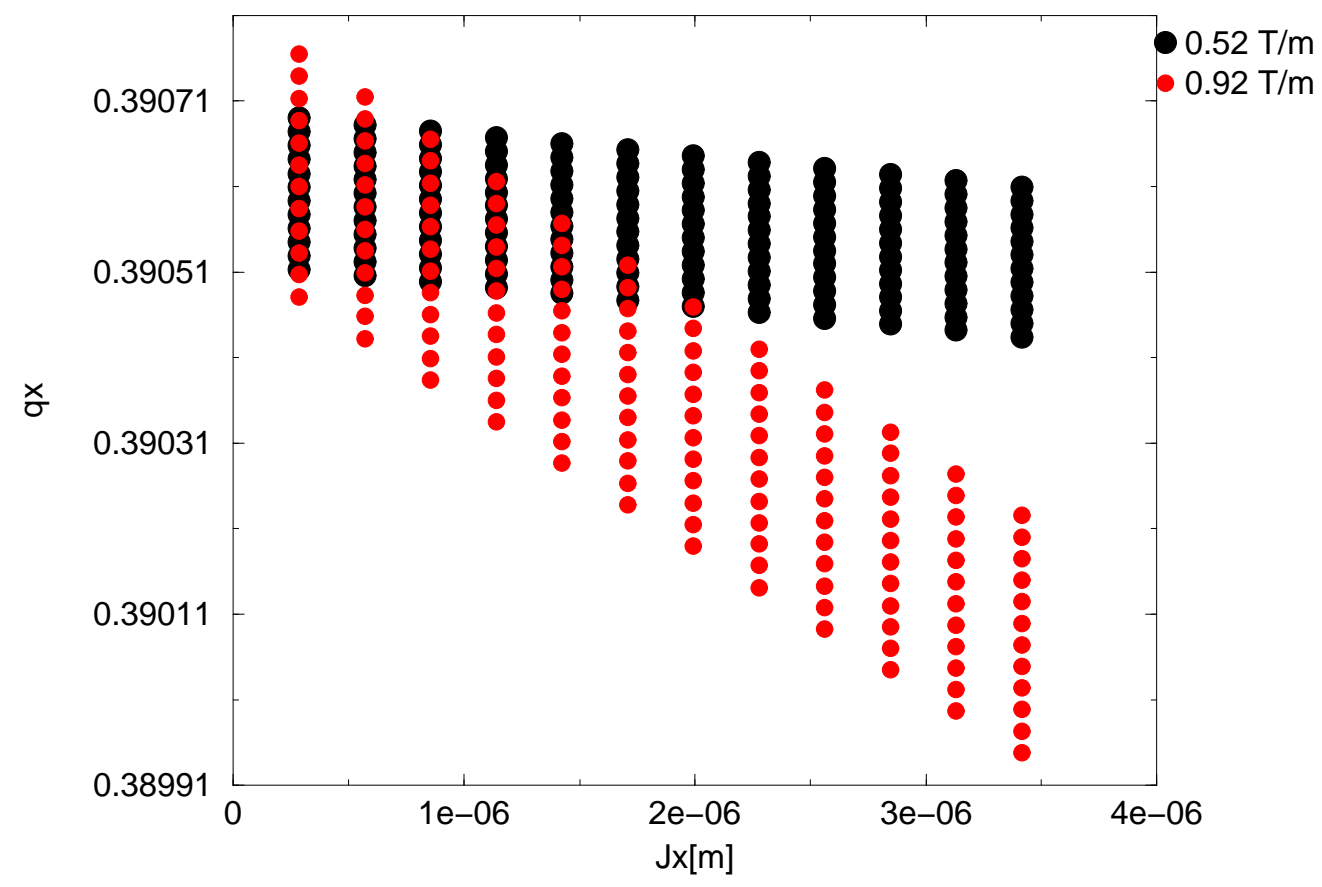

Figure 15.6: Horizontal tune distribution of the particles after tracking for 62530 turns. 


\section{Tune Spread}

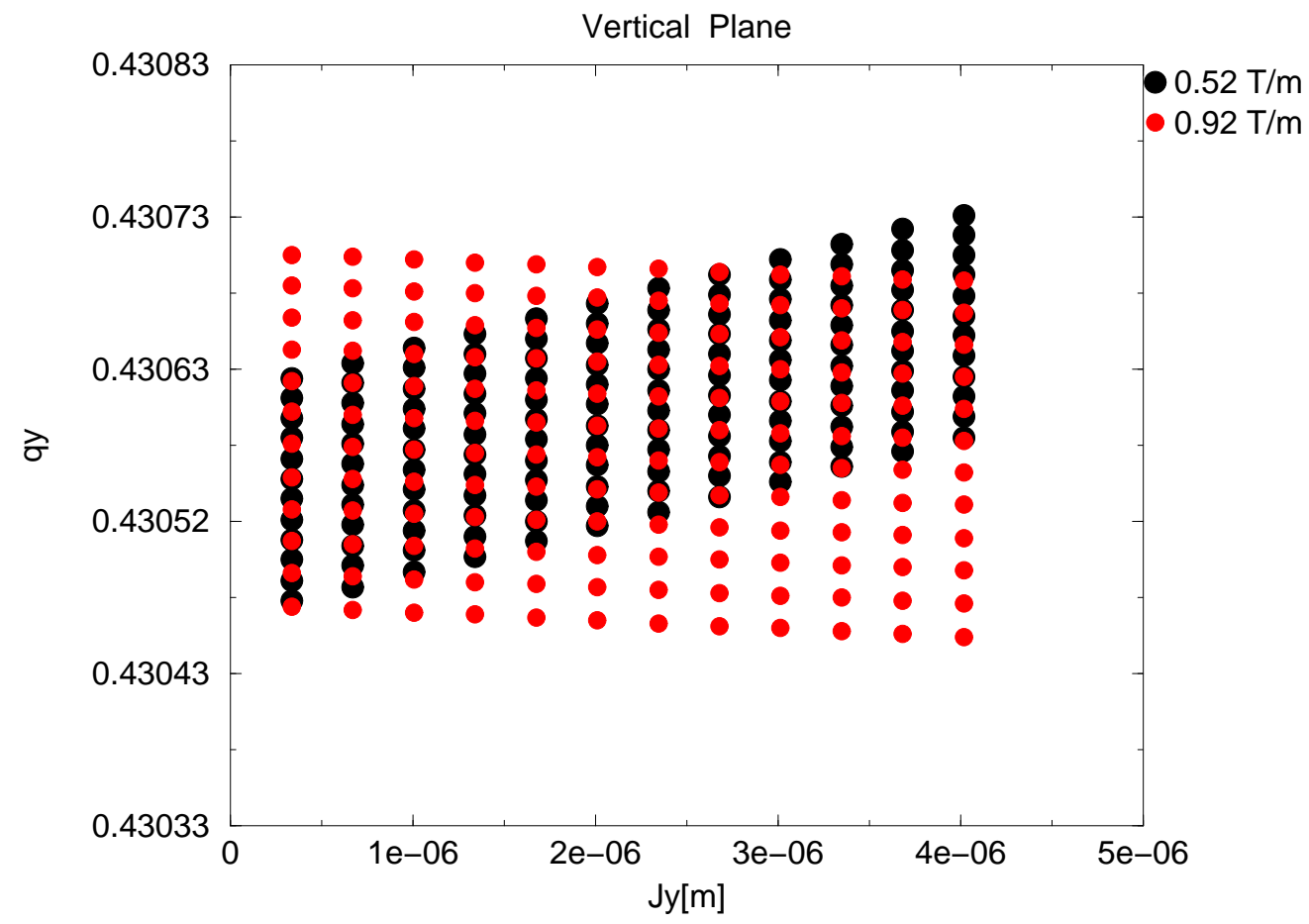

Figure 15.7: Vertical tune distribution of the particles after tracking for 62530 turns. 
dynamic aperture is not sensitive to this sextupole component in the range of sextupole strenghts used in this study. Even in the very extreme case in which the sextupole component is almost double the nominal strenght, the dynamic aperture although reduced is big enough to hold most of the particles of the beam.

It was also shown through Marylie simulations that the tune spread of the particles due to their different amplitudes is not significant and hence it doesn't represent any risk for the stable operation of the machine. The addition of sextupoles correctors to the RCMS design doesn't seem neccesary according to the results that has been presented in this chapter. 


\section{Appendix A}

\section{Multipolar Expansion}

The horizontal kick $\Delta x^{\prime}$ is produced either by misalignments and/or magnetic field errors that in general can be represented by a variation of the magnetic field $\Delta B$. The relation between $\Delta B_{x}$, the horizontal variation of the magnetic field and $\Delta B_{y}$, the vertical variation of the magnetic field with the horizontal and vertical kicks are given by:

$$
\begin{aligned}
\Delta x^{\prime} & =-\frac{l}{\rho}=-\frac{e \Delta B_{y} l}{p}, \\
\Delta y^{\prime} & =\frac{l}{\rho}=\frac{e \Delta B_{x} l}{p}
\end{aligned}
$$

where $\rho$ is the magnetic bending radius, $e$ is the electron charge, $l$ is the length of the magnet and $p$ is the momentum of the particle.

The magnetic field can be expanded in function of its multipole components with the expression ( see for example [2]):

$$
\Delta B_{y}+i \Delta B_{x}=B_{0} \sum_{n}\left(b_{n}+i a_{n}\right)(x+i y)^{n}
$$


where $a_{n}$ the skew multipolar component and $b_{n}$ the normal multipolar component. Eq. A.2 leads to:

$$
\begin{aligned}
\Delta x^{\prime}= & A_{1} y-B_{1} x+2 A_{2} x y+B_{2}\left(-x^{2}+y^{2}\right)+B_{3}\left(-x^{3}+3 x y^{2}\right)+ \\
& A_{3}\left(3 x^{2} y-y^{3}\right)+A_{4}\left(4 x^{3} y-4 x y^{3}\right)+B_{4}\left(-x^{4}+6 x^{2} y^{2}-y^{4}\right)+\ldots \\
\Delta y^{\prime}= & A_{1} x+B_{1} y+2 B_{2} x y+A_{2}\left(x^{2}-y^{2}\right)+A_{3}\left(x^{3}-3 x y^{2}\right)+ \\
& B_{3}\left(3 x^{2} y-y^{3}\right)+B_{4}\left(4 x^{3} y-4 x y^{3}\right)+A_{4}\left(x^{4}-6 x^{2} y^{2}+y^{4}\right)+\ldots
\end{aligned}
$$

where $A_{n}=\frac{e B_{0} l a_{n}}{p}$ and $B_{n}=\frac{e B_{0} l b_{n}}{p}$. 


\section{Appendix B}

\section{Equivalent Multipolar Coefficients in RHIC IRs}

It has been shown in Appendix A that a magnetic error at a particular magnet can be expressed in function of its multipolar components. Using the technique developed in this dissertation is only possible to measure the equivalent magnetic error for a group of magnets rather than for a single magnet. In such a case is still possible to expand the magnetic error in function of its multipoles components but they have to be redefined. The idea is that the contribution of a particular multipoles in all magnets contained in the set or package that is being studied can be expressed in a single multipole that will produce exactly the same effect (at least outside the package of magnets) when located at some particular point $s_{0}$.

Deductions for the equivalent skew quadrupole component, quadrupole gradient component, sextupole components and octupole components for a set of three magnets (RHIC triplets) were done in Mathematica Notebooks. 
The following sections show calculation for all the cited cases.

\section{B.1 Calculation of the Equivalent Skew Quadrupole for a RHIC triplet}

Skew quadrupole errors $A_{1}{ }^{a}, A_{1}{ }^{b}$ and $A_{1}{ }^{c}$ are assumed in each of the 3 magnets of the triplet. Such skew errors will start betatron oscillations (single turn analysis).

$$
\begin{aligned}
x(s)= & \sin \left(\psi_{x}-\psi_{x a}\right) A_{1}^{a} y_{a} \sqrt{\beta_{x}} \sqrt{\beta_{x a}}+ \\
& \sin \left(\psi_{x}-\psi_{x b}\right) A_{1}{ }^{b} y_{b} \sqrt{\beta_{x}} \sqrt{\beta_{x b}}+ \\
& \sin \left(\psi_{x}-\psi_{x c}\right) A_{1}^{c} y_{c} \sqrt{\beta_{x}} \sqrt{\beta_{x c}}
\end{aligned}
$$

where $\psi_{x}$ is short notation for $\psi_{x}(s)$, the horizontal phase advance, $\psi_{x} i$ is the phase advance in $i$ quadrupole of the triplet ( $i$ can be either $a, b$ or $c$ ), yi the vertical beam position in the $i$ quadrupole of the triplet, $\beta_{x}$ a short notation for $\beta_{x}(s)$ the horizontal beta functions and $\beta_{x i}$ is the horizontal beta function in the $i$ quadrupole of the triplet.

Here, it was assumed that the skew error in each quadrupole acts in a single point of the quadrupole or that the quadrupole is very thin compared to the total length of the triplet.

Assuming $J$ in the vertical plane doesn't change significantly in the length occupied by the set of magnets the vertical positions of the beam at each magnet $y_{a}, y_{b}$ and $y_{c}$ are given by:

$$
y_{a}=-\sqrt{2 J \beta_{y a}} \sin \left(\varphi-\psi_{y a}\right)
$$




$$
\begin{aligned}
& y_{b}=-\sqrt{2 J \beta_{y b}} \sin \left(\varphi-\psi_{y b}\right) \\
& y_{c}=-\sqrt{2 J \beta_{y c}} \sin \left(\varphi-\psi_{y c}\right)
\end{aligned}
$$

where $\varphi$ is some arbitrary phase where the vertical oscillation started. The other variables have the same meaning as their corresponding ones in the horizontal plane.

Substituting Eq. B.3 in Eq. B.2 and assuming that the phase advance for all the magnets in the package is the same (for RHIC triplets $\psi_{y a} \sim \psi_{y b} \sim \psi_{y c}$ ) it follows that:

$$
\begin{aligned}
x(s)= & -\sqrt{2} \sqrt{J} \sin \left(\psi_{x}-\psi_{x b}\right) \sin \left(\varphi-\psi_{y b}\right) \sqrt{\beta_{x}} \\
& \left(A_{1}^{a} \sqrt{\beta_{x 1}} \sqrt{\beta_{y 1}}+A_{1}^{b} \sqrt{\beta_{x b}} \sqrt{\beta_{y b}}+A_{1}^{c} \sqrt{\beta_{x c}} \sqrt{\beta_{y c}}\right),
\end{aligned}
$$

from Eq. B.4 it is easy to see that it is possible to write:

$$
x(s)=A_{1}^{e} y_{e} \sqrt{\beta_{e} \beta_{x}} \sin \left(\psi_{x}(s)-\psi_{x b}\right)
$$

where $y_{e}$ is the vertical position of the beam at the some position inside the triplet and is given by:

$$
y_{e}=\sqrt{2 J \beta_{y e}} \sin \left(\varphi-\psi_{y b}\right)
$$

where $\beta_{y e}$ is the vertical beta function at the longitudinal position $s_{0}$ Finally the equivalent skew quadrupole at $s_{0}$ is given by:

$$
A_{1}^{e}=\frac{A_{1}^{a} \sqrt{\beta_{x a}} \sqrt{\beta_{y a}}+A_{1}^{b} \sqrt{\beta_{x b}} \sqrt{\beta_{y b}}+A_{1}^{c} \sqrt{\beta_{x c}} \sqrt{\beta_{y c}}}{\sqrt{\beta_{x e}} \sqrt{\beta_{y e}}}
$$


$A_{1}{ }^{e}$ is symmetric in $\mathrm{x}$ and $\mathrm{y}$ as can be seen in the previous formula and hence $A_{1}{ }^{e}$ has the same value in both planes.

\section{B.2 Calculation of the Equivalent Skew Quadrupole for a RHIC IR}

Since equivalent skew quadrupole errors can be assigned to each triplet the calculation of the equivalent skew quadrupole error at the IR is reduced to calculate the equivalent skew error of two skew quadrupoles which can be done following exactly the same procedure as in the previous section. This leads to:

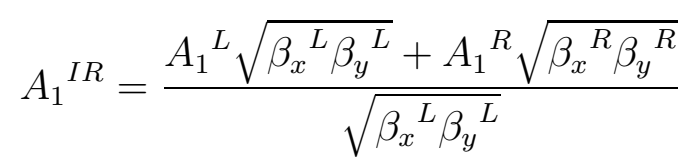

here, $A_{1}{ }^{I R}$ is the equivalent skew gradient for the whole IR, $A_{1}{ }^{L}$ and $A_{1}{ }^{R}$ are the equivalent skew gradients for the left and right triplets respectively, and the beta function with superscripts $L$ and $R$ are evaluated at the position of the left and right skew quadrupole corrector respectively. Since,

$$
\beta_{x}{ }^{L} \beta_{y}{ }^{L}=\beta_{x}{ }^{R} \beta_{y}{ }^{R}
$$

then,

$$
A_{1}{ }^{I R}=A_{1}{ }^{L}+A_{1}{ }^{R}
$$

Therefore the equivalent skew quadrupole error for the whole IR is equal to the sum of each individual triplet quadrupole errors.

This also means that it is possible to locally decouple an IR with only one skew quadrupole corrector and the strength of such corrector should be 
equal to the sum of strengths required in the skew quadrupole correctors in each side of the IR.

\section{B.3 Calculation of the Equivalent Quadrupole Gradient for a RHIC triplet}

Gradient errors $B_{1}{ }^{a}, B_{1}{ }^{b}$ and $B_{1}{ }^{c}$ are assumed in all magnet of the triplet. Such gradients (single turn analysis) will give rise to betatron oscillations given by:

$$
\begin{aligned}
x(s)= & \sqrt{\beta_{x}} \sin \left(\psi_{x}-\psi_{x a}\right) x_{a} \sqrt{\beta_{x a}} B_{1}{ }^{a}+ \\
& \sqrt{\beta_{x}} \sin \left(\psi_{x}-\psi_{x b}\right) x_{b} \sqrt{\beta_{x b}} B_{1}{ }^{b}+ \\
& \sqrt{\beta_{x}} \sin \left(\psi_{x}-\psi_{x c}\right) x_{c} \sqrt{\beta_{x_{c}}} B_{1}{ }^{c}
\end{aligned}
$$

where the notation is the same used in the previous sections. Assuming that $J$ doesn't change significantly in the the triplet it is possible to write:

$$
\begin{aligned}
& x_{a}=-\sqrt{2 J \beta_{x a}} \sin \left(\varphi-\psi_{x a}\right) \\
& x_{b}=-\sqrt{2 J \beta_{x b}} \sin \left(\varphi-\psi_{x b}\right) \\
& x_{c}=-\sqrt{2 J \beta_{x_{c}}} \sin \left(\varphi-\psi_{x c}\right)
\end{aligned}
$$

where $\varphi$ is some arbitrary phase where the biggest source of betatron oscillation starts. Since the phases at the triplets are almost the same, Eq. B.11 in Eq. B.12 leads to:

$$
x(s)=-\sqrt{2} \sqrt{J} \sqrt{\beta_{x}} \sin \left(\varphi-\psi_{x b}\right) \sin \left(\psi-\psi_{x b}\right)
$$




$$
* \quad\left(\beta_{x_{a}} B_{1}{ }^{a}+\beta_{x b} B_{1}^{b}+\beta_{x_{c}} B_{1}^{c}\right)
$$

substitution of

$$
\frac{x_{e}}{\sqrt{\beta_{x_{e}}}}=-\sqrt{2 J} \sin \left(\varphi-\psi_{x b}\right)
$$

in Eq. equi:grad:orb finally leads to:

$$
x(s)=\sqrt{\beta_{x} \beta_{x e}} B_{1}^{x e} x_{e} \sin \left(\psi_{x a}-\psi_{x}\right)
$$

where the equivalent gradient $B_{1}{ }^{x e}$ is given by:

$$
B_{1}^{x e}=\frac{\beta_{x a} B_{1}{ }^{a}+\beta_{x b} B_{1}^{b}+\beta_{x c} B_{1}^{c}}{\beta_{x e}}
$$

Following a completely analogous process the equivalent gradient error $B_{1}^{\text {ye }}$ in the vertical plane is given by:

$$
B_{1}^{y e}=\frac{\beta_{y_{a}} B_{1}{ }^{a}+\beta_{y_{b}} B_{1}{ }^{b}+\beta_{y_{c}} B_{1}{ }^{c}}{\beta_{y e}}
$$

Even though gradient errors of individual quadrupoles are the same in both planes the equivalent gradients for the whole triplet are different due to the different values of the beta functions in both planes. 


\section{B.4 Calculation of the Equivalent Normal Sextupole for a RHIC triplet}

Betatron oscillations produced by $B_{2}{ }^{a}, B_{2}{ }^{b}$ and $B_{2}{ }^{c}$, the normal sextupoles errors in each of the quadrupoles of a RHIC triplet are given by:

$$
\begin{aligned}
x(s)= & 2 B_{2}^{a} \sin \left(\psi_{x}-\psi_{x a}\right)\left(-x_{a}{ }^{2}+y_{a}{ }^{2}\right) \sqrt{\beta_{x}} \sqrt{\beta_{x a}} \\
& +2 B_{2}^{b} \sin \left(\psi_{x}-\psi_{x b}\right)\left(-x_{b}{ }^{2}+y_{b}{ }^{2}\right) \sqrt{\beta_{x}} \sqrt{\beta_{x b}} \\
& +2 B_{2}{ }^{c} \sin \left(\psi_{x}-\psi_{x c}\right)\left(-x_{c}{ }^{2}+y_{c}{ }^{2}\right) \sqrt{\beta_{x}} \sqrt{\beta_{x c}}
\end{aligned}
$$

where we have used the same notation as in the previous sections.

Assuming $J$ doesn't change significantly when going through the interaction region it is possible to write:

$$
\begin{aligned}
& x_{a}=\sqrt{2 J_{x} \beta_{x_{a}}} \sin \left(\psi_{x_{a}}-\varphi_{x}\right) \\
& x_{b}=\sqrt{2 J_{x} \beta_{x b}} \sin \left(\psi_{x b}-\varphi_{x}\right) \\
& y_{a}=\sqrt{2 J_{y} \beta_{y_{a}}} \sin \left(\psi_{y_{a}}-\varphi_{y}\right) \\
& x_{b}=\sqrt{2 J_{y} \beta_{y_{b}}} \sin \left(\psi_{y_{b}}-\varphi_{y}\right)
\end{aligned}
$$

where the variables $J_{x}$ the action in the horizontal plane, $J_{y}$ the action in the vertical plane, $\varphi_{x}$ the phase associated with the horizontal betatron oscillation and $\varphi_{y}$ the phase associated with the vertical betatron oscillation have been introduced.

If the phase advance is the same in all quadrupoles of the triplet then 
Eq. B.18 can be simplified to:

$$
\begin{aligned}
x(s)= & -4 J \sin \left(\varphi_{x}-\psi_{x b}\right)^{2} \sin \left(\psi_{x}-\psi_{x b}\right) \sqrt{\beta_{x}}\left(B_{2}{ }^{a} \beta_{x a}{ }^{\frac{3}{2}}+B_{2}{ }^{b} \beta_{x b}{ }^{\frac{3}{2}}+B_{2}{ }^{c} \beta_{x c^{\frac{3}{2}}}\right) \\
& +4 J \sin \left(\psi_{x}-\psi_{x b}\right) \sin \left(\varphi_{y}-\psi_{y b}\right)^{2} \sqrt{\beta_{x}} B_{2}{ }^{a} \sqrt{\beta_{x a}} \beta_{y a}+ \\
& +4 J \sin \left(\psi_{x}-\psi_{x b}\right) \sin \left(\varphi_{y}-\psi_{y b}\right)^{2}\left({B_{2}}^{b} \sqrt{\beta_{x b}} \beta_{y b}+B_{2}{ }^{c} \sqrt{\beta_{x c}} \beta_{y c}\right)
\end{aligned}
$$

As in the other cases, the formula should expressed in function of beam positions $x_{e}$ and $y_{e}$ at the place where the equivalent coefficients want to be calculated.

$$
\begin{aligned}
x(s)= & -\frac{\sin \left(\psi_{x}-\psi_{x b}\right) x_{e}{ }^{2} \sqrt{\beta_{x}}\left(B_{2}{ }^{a} \beta_{x a^{\frac{3}{2}}}+B_{2}{ }^{b} \beta_{x b^{2}}{ }^{\frac{3}{2}}+B_{2}{ }^{c} \beta_{x c}{ }^{\frac{3}{2}}\right)}{\beta_{x e}} \\
& +\frac{\sin \left(\psi_{x}-\psi_{x b}\right) y_{e}{ }^{2} \sqrt{\beta_{x}}\left(B_{2}^{a} \sqrt{\beta_{x a}} \beta_{y a}+B_{2}^{b} \sqrt{\beta_{x b}} \beta_{y b}+B_{2}{ }^{c} \sqrt{\beta_{x c}} \beta_{y c}\right)}{\beta_{y e}}
\end{aligned}
$$

It is then now possible to write these expressions in function of their equivalent normal sextupoles:

$$
x(s)=2 \sin \left(\psi_{x}-\psi_{x b}\right)\left(-B_{2}{ }^{x a} x_{e}{ }^{2}+B_{2}{ }^{x b} y_{e}^{2}\right) \sqrt{\beta_{x} \beta(s)}
$$

where $B_{2}{ }^{x a}$ and $B_{2}{ }^{x b}$ are the equivalent normal sextupole coefficients in the $\mathrm{x}$ plane and they are given by:

$$
\begin{aligned}
& B_{2}^{x a}=\frac{B_{2}^{a} \beta_{x a^{\frac{3}{2}}}+B_{2}^{b} \beta_{x b^{\frac{3}{2}}}+B_{2}^{c} \beta_{x c^{\frac{3}{2}}}}{\beta_{x e^{\frac{3}{2}}}} \\
& B_{2}{ }^{x b}=\frac{B_{2}{ }^{a} \sqrt{\beta_{x a}} \beta_{y a}+B_{2}^{b} \sqrt{\beta_{x b}} \beta_{y b}+B_{2}^{c} \sqrt{\beta_{x c}} \beta_{y c}}{\sqrt{\beta_{x e}} \beta_{y e}}
\end{aligned}
$$


The betatron equations in the vertical plane are:

$$
\begin{aligned}
y(s)= & 2 B_{2}^{a} \sin \left(\psi_{y}-\psi_{y a}\right) x_{a} y_{a} \sqrt{\beta_{y}} \sqrt{\beta_{y a}}+ \\
& 2 B_{2}^{b} \sin \left(\psi_{y}-\psi_{y b}\right) x_{b} y_{b} \sqrt{\beta_{y}} \sqrt{\beta_{y b}}+ \\
& 2 B_{2}^{c} \sin \left(\psi_{y}-\psi_{y c}\right) x_{c} y_{c} \sqrt{\beta_{y}} \sqrt{\beta_{y c}}
\end{aligned}
$$

Same assumptions as for the horizontal plane are done here to obtain:

$$
\begin{aligned}
y(s) & =4 J \sin \left(\varphi_{x}-\psi_{x b}\right) \sin \left(\varphi_{y}-\psi_{y b}\right) \sin \left(\psi_{y}-\psi_{y b}\right) \\
& * \sqrt{\beta_{y}}\left({B_{2}}^{a} \sqrt{\beta_{x a}} \beta_{y a}+B_{2}^{b} \sqrt{\beta_{x b}} \beta_{y b}+B_{2}^{c} \sqrt{\beta_{x c}} \beta_{y c}\right)
\end{aligned}
$$

Expressing the Eq. B.25 in function of their beam positions at the place where the equivalent coefficients want to be calculated results in:

$$
y(s)=\sqrt{\beta_{y}(s) \beta_{y e}} B_{2}{ }^{y} x_{e} y_{e} \sin \left(\psi_{y}(s)-\psi_{b}\right)
$$

where the equivalent normal sextupole in the y plane $B_{2}^{y}$ is given by:

$$
B_{2}^{y}=\frac{B_{2}{ }^{a} \sqrt{\beta_{x a}} \beta_{y a}+B_{2}{ }^{b} \sqrt{\beta_{x b}} \beta_{y b}+B_{2}^{c} \sqrt{\beta_{x c}} \beta_{y c}}{\sqrt{\beta_{x e}} d \beta_{y e}}
$$

This coefficient is exactly the same as $B_{2}{ }^{x b}$ which means that 2 independent coefficients are enough to completely describe an equivalent normal sextupole coefficient. 


\section{B.5 Calculation of the Equivalent Skew Sex- tupole for a RHIC Triplet}

Calculation for the skew sextupole coefficients are very similar to the preceding ones. The equivalent skew sextupole coefficient in the horizontal plane, $A_{2}{ }^{x}$, exactly resembles the algebra done for the equivalent normal sextupole coefficient in the horizontal plane and is given by:

$$
A_{2}^{x}=\frac{A_{2}^{a} \beta_{x a} \sqrt{\beta_{y a}}+A_{2}^{b} \beta_{x b} \sqrt{\beta_{y b}}+A_{2}^{c} \beta_{x c} \sqrt{\beta_{y c}}}{\beta_{x e} \sqrt{\beta_{y e}}}
$$

In the vertical plane two different equivalent coefficients arise and they are equal to:

$$
\begin{aligned}
& A_{2}^{y a}=\frac{A_{2}{ }^{a} \beta_{x a} \sqrt{\beta_{y a}}+A_{2}^{b} \beta_{x b} \sqrt{\beta_{y b}}+A_{2}^{c} \beta_{x c} \sqrt{\beta_{y c}}}{\beta_{x e} \sqrt{\beta_{y e}}} \\
& A_{2}^{y b}=\frac{A_{2}^{a} \beta_{y a^{\frac{3}{2}}}+A_{2}^{b} \beta_{y b^{\frac{3}{2}}}+A_{2}^{c} \beta_{y c}{ }^{\frac{3}{2}}}{\beta_{y e^{\frac{3}{2}}}}
\end{aligned}
$$

As for the equivalent normal sextupole coefficients, there are two identical coefficients and then only two coefficients are needed to completely characterize an equivalent skew sextupole coefficient.

\section{B.6 Equivalent Octupole Coefficients}

The calculation of the equivalent coefficients for octupoles is very similar to the previous calculations and only the results will be shown here. 
The equivalent skew octupole coefficients in the x plane are given by:

$$
\begin{aligned}
& A_{3}{ }^{x b}=-\left(\frac{A_{3}{ }^{a} \sqrt{\beta_{x a}} \beta_{y a^{\frac{3}{2}}}+A_{3}{ }^{b} \sqrt{\beta_{x b}} \beta_{y b^{\frac{3}{2}}}+A_{3}{ }^{c} \sqrt{\beta_{x c}} \beta_{y c}{ }^{\frac{3}{2}}}{\sqrt{\beta_{x e}} \beta_{y e^{\frac{3}{2}}}}\right) \\
& A_{3}{ }^{x a}=-\left(\frac{A_{3}{ }^{a} \beta_{x a^{\frac{3}{2}}} \sqrt{\beta_{y a}}+A_{3}^{b} \beta_{x b^{\frac{3}{2}}} \sqrt{\beta_{y b}}+A_{3}^{c} \beta_{x c^{\frac{3}{2}}} \sqrt{\beta_{y c}}}{\beta_{x e^{\frac{3}{2}}} \sqrt{\beta_{y e}}}\right)
\end{aligned}
$$

while the equivalent skew octupole coefficients in the y plane are given by:

$$
\begin{aligned}
& A_{3}{ }^{y a}=-\left(\frac{A_{3}{ }^{a} \beta_{x a^{\frac{3}{2}}} \sqrt{\beta_{y a}}+A_{3}{ }^{b} \beta_{x b^{\frac{3}{2}}} \sqrt{\beta_{y b}}+A_{3}{ }^{c} \beta_{x c^{\frac{3}{2}} \sqrt{\beta_{y c}}}}{\beta_{x e^{\frac{3}{2}} \sqrt{\beta_{y e}}}}\right) \\
& A_{3}{ }^{y b}=-\left(\frac{A_{3}{ }^{a} \sqrt{\beta_{x a}} \beta_{y a^{\frac{3}{2}}}+A_{3}{ }^{b} \sqrt{\beta_{x b}} \beta_{y b^{\frac{3}{2}}}+A_{3}{ }^{c} \sqrt{\beta_{x c}} \beta_{y c}{ }^{\frac{3}{2}}}{\sqrt{\beta_{x e}} \beta_{y e^{\frac{3}{2}}}}\right)
\end{aligned}
$$

Since 2 pairs of these coefficients are equal only 2 coefficients are needed to completely describe the equivalent skew octupole coefficients. Similarly for the equivalent normal octupole coefficients in the x plane:

$$
\begin{aligned}
B_{3}{ }^{x a} & =-\left(\frac{{B_{3}}^{a} \beta_{x a}{ }^{2}+B_{3}{ }^{b} \beta_{x b}{ }^{2}+B_{3}^{c} \beta_{x c}{ }^{2}}{\beta_{x e}{ }^{2}}\right) \\
B_{3}{ }^{x b} & =-\left(\frac{B_{3}{ }^{a} \beta_{x a} \beta_{y a}+B_{3}{ }^{b} \beta_{x b} \beta_{y b}+B_{3}{ }^{c} \beta_{x c} \beta_{y 3}}{\beta_{x e} \beta_{y e}}\right)
\end{aligned}
$$

and in the y plane:

$$
B_{3}{ }^{y a}=-\left(\frac{B_{3}{ }^{a} \beta_{x a} \beta_{y a}+B_{3}^{b} \beta_{x b} \beta_{y b}+B_{3}{ }^{c} \beta_{x c} \beta_{y c}}{\beta_{x e} \beta_{y e}}\right)
$$




$$
B_{3}{ }^{y b}=-\left(\frac{B_{3}{ }^{a} \beta_{y a}{ }^{2}+B_{3}{ }^{b} \beta_{y b}{ }^{2}+B_{3}{ }^{c} \beta_{y c}{ }^{2}}{\beta_{y e}{ }^{2}}\right)
$$

Since two of the coefficients are equal only 3 coefficients are needed.

In summary, to completely describe the equivalent octupole coefficients 5 variables are needed. It is shown in the Section 5.5 that the beam based method to find linear and nonlinear coefficients is able to discern up to 4 different unknowns. Determination of octupoles would require further studies to find if a simple modification of the technique to find linear and non linear error would work in this case.

\section{B.7 Multipolar Expansion for an Equivalent Magnetic Kick in a RHIC Triplet}

As a summary of the previous sections it was found that 3 independent coefficient were needed to completely describe the equivalent linear errors (unlike the case of a single magnet where only two coefficients are needed), and a total of four independent coefficients to completely describe the equivalent sextupole errors (unlike the case of a single magnet where only 2 coefficients are needed). According to the previous result the equivalent magnetic kick can be expanded in its equivalent multipolar components in the following way:

$$
\Delta x^{\prime e q}=A_{1}^{e q} y_{0}-B_{1}^{x} x_{0}+2 A_{2}{ }^{x} x_{0} y_{0}-B_{2}{ }^{x a} x_{0}^{2}+B_{2}{ }^{y} y_{0}^{2}+\ldots
$$




$$
\Delta y^{\prime e q}=A_{1}^{e q} x_{0}+B_{1}^{y} y_{0}+2 B_{2}{ }^{y} x_{0} y_{0}+A_{2}{ }^{x} x_{0}^{2}-A_{2}{ }^{y b} y_{0}^{2}+\ldots
$$

This will be the expression used to evaluate errors from the orbit measurements as is described in Chapters 5 and Chapter 7. 


\section{Appendix $\mathrm{C}$}

\section{Fits of Orbit Data to the Betatron Equation in RHIC Arcs}

It is clear from Chapter 2 that the biggest lattice errors in the early commissioning of RHIC were present at the Interaction Regions. As was shown in Chapter 3 and Chapter 5, it is possible to determine the magnitude of such error from the orbit in the arc immediately before the IR under study an the orbit in the arc immediately after the mentioned IR.

Three different methods were implemented in $\mathrm{C}++$ programming language to do the mentioned task.

The first method is the simplest one can imagine. Since action and phase are roughly constant in the arcs, an average of actions and phases are good estimates of the real action and phase in the arcs. Those averages are then used in:

$$
\Delta x^{\prime}=\sqrt{\frac{2 J_{R}+2 J_{L}-4 * \sqrt{J_{R}} \sqrt{J_{L}} \cos \left(\psi_{R}-\psi_{L}\right)}{\beta\left(s_{0}\right)}}
$$


where $\Delta x^{\prime}$ is a quantity that can be related with the errors in the IR as was discussed in Chapter 3.

The subroutine for this first method has been developed in such a way that it is able to reject values beyond $2 \sigma$ from the average. In this way it is possible to eliminate a significant amount of noise. The name of the subroutine is FITTED_POS because was initially created to find the position of the beam at the skew quadrupole corrector by fitting the data in the arc previous to the IR.

The second subroutine (BP_CORRECTOR) uses the average angle found with the previous subroutine and vary the action until the model data is as close as possible to the real data. This procedure was born from the observation that the phase graphs have much less variations or noise than the corresponding actions graphs. Different to the previous subroutine, this subroutine uses a simple formula (Eq. C.2) to determine the amplitude of the orbit in a particular arc and there are no rejection of points. That makes this algorithm faster than the previous one but more susceptible to errors.

$$
\begin{aligned}
\text { amplitude } & =\frac{\sum_{i} y_{i} f\left(x_{i}\right)}{f\left(x_{i}\right)^{2}} \\
& =\frac{\sum_{i} y_{i} \sqrt{\beta(i)} \sin (\operatorname{mux}(i)-\varphi)}{\beta(i) \sin ^{2}(\operatorname{mux}(i)-\varphi)}
\end{aligned}
$$

The third subroutine (NEW_FIT) fits the data to a model:

$$
x(s)=a \sin (\operatorname{mux}(i))+b \sin (\operatorname{mux}(i))
$$


where $a$ and $b$ are the variables to fit the data. As before standard formulas are used to find the variables from the data and there is no rejection algorithm. Those formulas are:

$$
\begin{aligned}
& a=(x s * c 2-s c * x c) /(s 2 * c 2-s c * s c) \\
& b=(s 2 * x c-x s * s c) /(s 2 * c 2-s c * s c)
\end{aligned}
$$

where:

$$
\begin{aligned}
x s & =\sum_{i} y_{i} \sqrt{\beta(i)} \sin (\operatorname{mux}(i)) \\
x c & =\sum_{i} y_{i} \sqrt{\beta(i)} \cos (\operatorname{mux}(i)) \\
s 2 & =\sum_{i} \beta(i) \sin ^{2}(\operatorname{mux}(i)) \\
c 2 & =\sum_{i} \beta(i) \cos ^{2}(\operatorname{mux}(i)) \\
s c & =\sum_{i} \beta(i) \sin (\operatorname{mux}(i)) \cos (\operatorname{mux}(i))
\end{aligned}
$$

The three subroutines were used to analyze an orbit in order to evaluate how close was the fit in the 3 cases to the real data. With this purpose in mind, an unnormalized $\chi^{2}$ was defined as:

$$
\chi^{2}=\left(y_{i}-f\left(x_{i}\right)\right)^{2}
$$

The analysis were performed in the vertical plane where the signal is stronger. Arcs that goes from IR12 to IR2 and IR2 to IR4 were analyzed independently. The three subroutines were used to fit the orbit data to the 


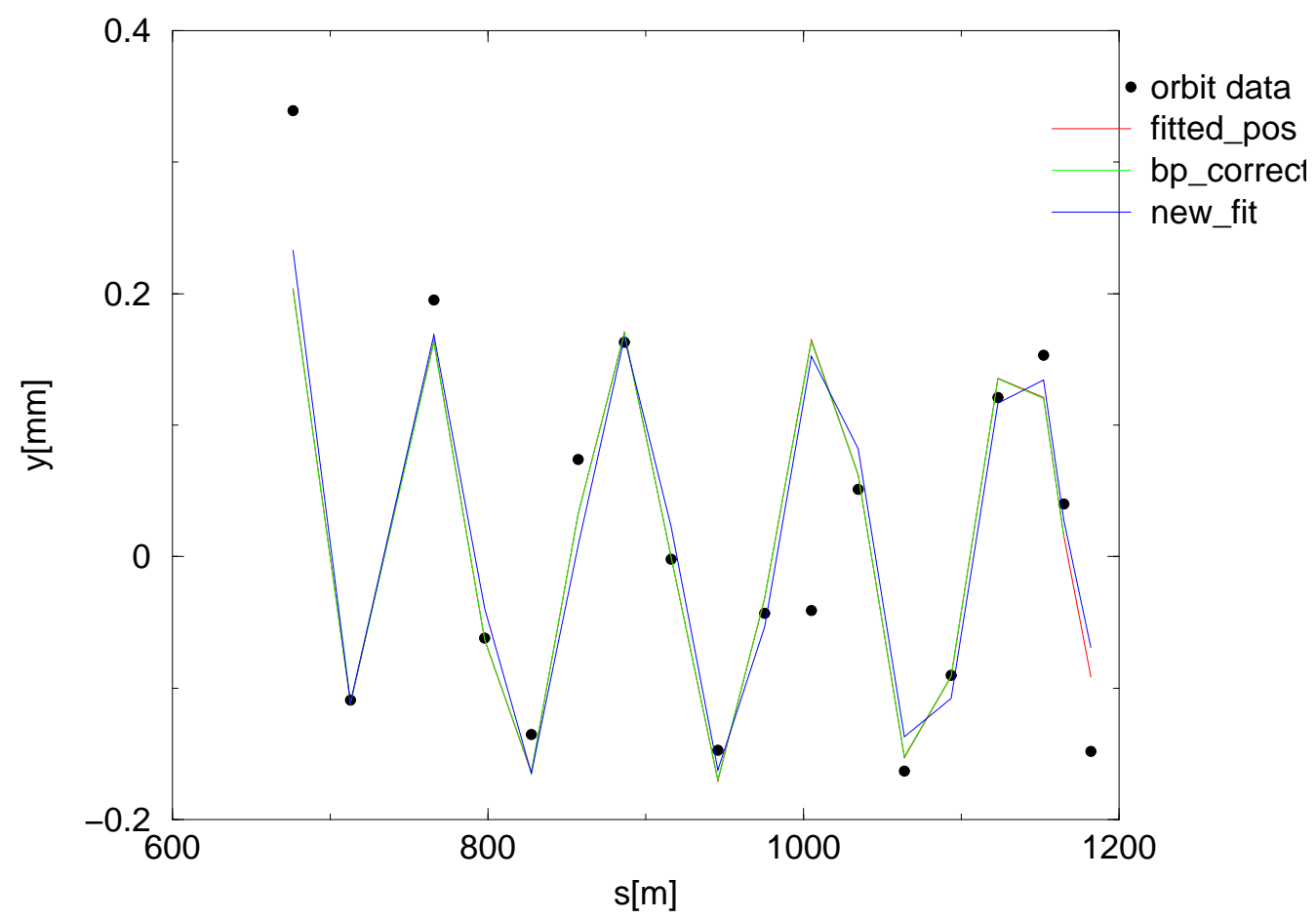

Figure C.1: The orbit data (weak signals)in the arc that goes from IR8 to IR10 of the previous figure is compared with the modeled orbits obtained with three different methods.

corresponding model in each case and the $\chi^{2}$ was evaluated. $\chi^{2}$ was exactly the same (within computer precision) when the results of all 3 subroutines were compared independently in each arc.

Weaker signals, on the other hand, have slight variations in the value of $\chi^{2}$ which is evident in Fig C.1 where orbit in arc that goes from IR8 to IR10 is compared with all three different fitting procedures. The marginal variations in $\chi^{2}$ can be seen on Table C.1

It is no clear by know if such a small variation in $\chi^{2}$ will have a significant 


\begin{tabular}{|l|c|l|}
\hline \multicolumn{1}{|c|}{ Routine } & $\chi^{2}$ & \multicolumn{1}{c|}{ Description } \\
\hline FITTED_POS & 0.0701 & $\begin{array}{l}\text { Average of action } \\
\text { and phase in the arcs } \\
\text { Bmplitude of model is } \\
\text { fitted to orbit with } \\
\text { constant phase obtained } \\
\text { from the previous routine } \\
\text { The equation } a \sin (\text { mux })+b \cos (\text { mux }) \\
\text { is fitted to orbit data. }\end{array}$ \\
\hline
\end{tabular}

Table C.1: Effectiveness of the fit for 3 different procedures on the same orbit data.

effect in the determination of linear or non linear errors. I suspect that the effect in the linear errors might be insignificant but no in the nonlinear errors. It easy (although not straightforward) to test this effect in the determination of linear errors. The effect on nonlinear errors determination is at this time uncertain since the method has not been completely developed.

Weak signals were used to do some of the analysis in Chapter 3 and Chapter 5. Future experiment will not require weak signals and then there is no any difference in using any or other subroutine to do the fits. 


\section{Appendix D}

\section{The Orbit Bump Method}

In order to evaluate the amount of coupling at the IRs, orbit bumps were done around several triplets during the RHIC 2000 run ([16] and [19]). Orbit bumps in either the vertical or the horizontal plane induces an oscillation in the perpendicular plane (see Fig. D.1) . A horizontal orbit bump will induce the vertical betatron oscillation

$$
y=\frac{\beta_{y}(s)}{2 \sin \pi \nu_{y}} \frac{A_{x}}{\sqrt{\beta_{x}^{d c}}} \cos \left(\left|\psi_{y}\right|-\pi \nu_{y}\right) \int_{\text {triplet }} k \sqrt{\beta_{x} \beta_{y}} d s
$$

where $\nu_{y}$ is the betatron tune, $\beta_{y}(s)$ are the vertical beta functions, $A_{x}$ is the orbit excursion at the position of the horizontal dipole corrector in the triplet, $\beta_{x}^{d c}$ is the horizontal beta function at the position of the triplet horizontal dipole corrector, $\kappa$ is the coupling error function along the triplet that

must be integrated with weight $\sqrt{\beta_{x} \beta_{y}}$, the square root of the product of the beta functions in both planes along the triplet, and $\psi_{y}$ is the phase advance.

Since all the vertical BPMs have the same beta functions, the rms value 

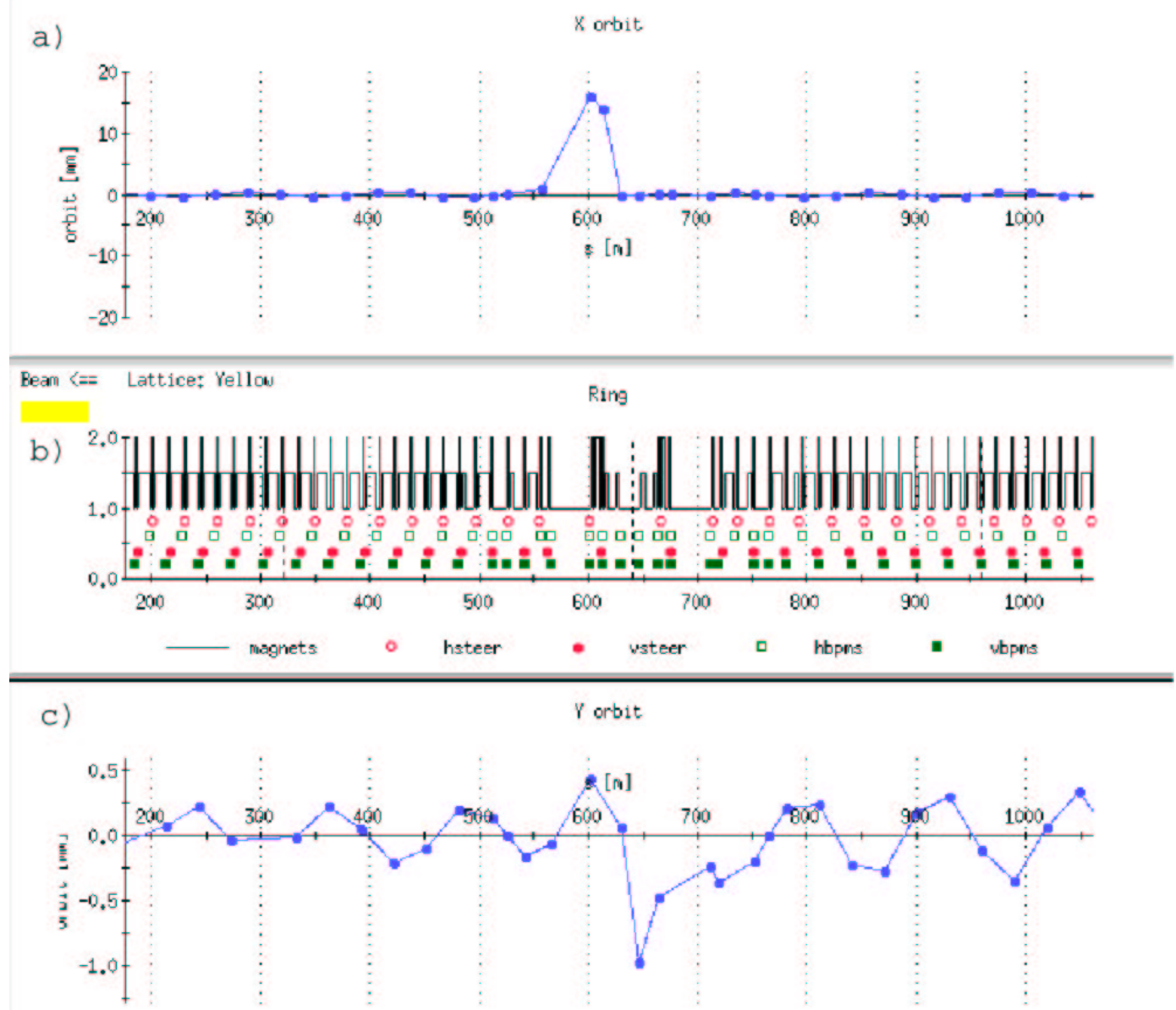

Figure D.1: a) A closed orbit bump about the left triplet of IR8 is built in the horizontal plane. b) Lattice representation of RHIC. c) Coupling in the triplet induces an orbit oscillation in the vertical plane. The rms value of this betatron oscillation can be used to calculate the skew quadrupole error. 
of Eq. D.1 is given by:

$$
y_{r m s}=\frac{\sqrt{\beta_{y}^{b p m}} A_{x}}{2 \sqrt{2}\left|\sin \pi \nu_{y}\right| \sqrt{\beta_{x}^{d c}}} \int \kappa \sqrt{\beta_{x} \beta_{y}} d s=\lambda A_{x}
$$

Since $A_{x}$ and $y_{r m s}$ are quantities that can be measured $\kappa$ can be determined to finally find the quadrupole integrated corrector strength required to compensate for the coupling error as follows:

$$
(k l)_{s c}=-\frac{\lambda}{\sqrt{\beta_{x}^{s c}} \sqrt{\beta_{y}^{s c}}} \frac{2 \sqrt{2 \beta_{x}^{d c}}\left|\sin \pi \nu_{y}\right|}{\sqrt{\beta_{y}^{b p m}}} .
$$

The following table summarize the estimated corrector strengths that were performed in both rings of RHIC before the correction had taken place. These

\begin{tabular}{|c|c|r|r|}
\hline IR & $\begin{array}{c}\text { Triplet } \\
\text { Blue/Yellow }\end{array}$ & $\begin{array}{r}\text { Blue corr.str. } \\
\left(10^{-3} 1 / \mathrm{m}\right)\end{array}$ & $\begin{array}{c}\text { Yellow corr.str. } \\
\left(10^{-3} 1 / \mathrm{m}\right)\end{array}$ \\
\hline IR2 & I1/O1 & $-0.22 \pm 0.16$ & 0.30 \\
& O2/I2 & $1.23 \pm 0.15$ & 0.76 \\
\hline IR6 & I5/O5 & 0.39 & $-0.94 \pm 0.08$ \\
& O6/I6 & 0.12 & $\pm 0.36 \pm 0.02$ \\
\hline IR8 & O7/I7 & -0.84 & $0.36 \pm 0.04$ \\
& I8/O8 & 1.32 & -1.10 \\
\hline
\end{tabular}

Table D.1: Calculated IR skew corrector strengths.

results can be compared with the ones obtained in Table 3.2. In order to do that, left and right triplet strengths in Table D.1 should be added according to Eq. B.9. We then have that at IR6 the sum of the skew triplet errors leads to 0.51 which is reasonably close to the integrated value of 0.61 in Table 3.2. Similarly for IR8 the sum of the individual skew quadrupole errors is equal to 0.48 compared to the integrated strength 0.67 obtained in Table 3.2. Finally 
IR2 individual skew quadrupole triplet errors add to 1.01 compared to 0.99 in Table 3.2. 


\section{Appendix E}

\section{Common problems found in the Action and Phase Analysis of Difference Orbits}

It is shown in this dissertation how the action and phase analysis can be used to evaluate errors at the IRs and to make calibration of the IR correctors.However, before doing any estimate from the action and phase obtained from the BPM measurements it is neccesary to make some preliminary analysis. Table E.1 is an example of the procedure that must be done before doing any numerical estimate.

\begin{tabular}{|c|c|c|c|c|c|}
\hline Corr. & Fit & $\begin{array}{c}\text { Phase } \\
\text { (Deg. })\end{array}$ & bx BPMs & \multicolumn{2}{|c|}{ b3 BPMs } \\
\cline { 5 - 6 } & & & Left & Right \\
\hline bo3-th10 & Ok. & 42 & Yes & Yes & No \\
bo2-th16 & Ok. & 35 & Yes & Yes & No \\
bo6-tv15 & Ok. & 76 & Yes & Yes & No \\
bo7-tv11 & Poor & 63 & Yes & Yes & No \\
\hline
\end{tabular}

Table E.1: Preliminary analysis of difference orbits used to evaluate triplet errors at 8'o clock IR. Only analysis in the horizontal plane are shown. 
The first column contains the name of dipole corrector used to excite the betatron oscillation or the desired shift of the closed orbit.

The second column indicates the quality of the fit of the data to the model, before and after the IR. The quality of the fit is evaluated visually. The fit of Fig. 3.1 is considered excellent in both arcs, before and after the IR while the fit of Fig. E.1 is considered poor. The third column shows a phase that measure the distance in degrees from the skew quadrupole corrector to the closest maximum of the orbit. The ideal distance is closer to 0 degrees as was discussed in Section 5.4.

The fourth column indicates if the central BPMs of the IR (bxs) are present or not. If they are not present it is not possible to make error estimations individually for each triplet of the IR.

The fifth column indicates if the two BPMs just outside the triplets (b3s) are present or not. These BPMs are the ones that are used to estimate the beam position at the correctors. If any of the BPMs fail then the closest BPM to the original one and closer to the IP (b1) is used instead.

The orbit shown in Fig. E.2 shows the ideal situation when all the six BPMs are working at 8 o'clock. In Fig. E.1 only three of those BPMs are working which makes impossible to calculate errors for the each triplet individually and all that it is possible to obtain is an overall error estimation for the whole IR. 

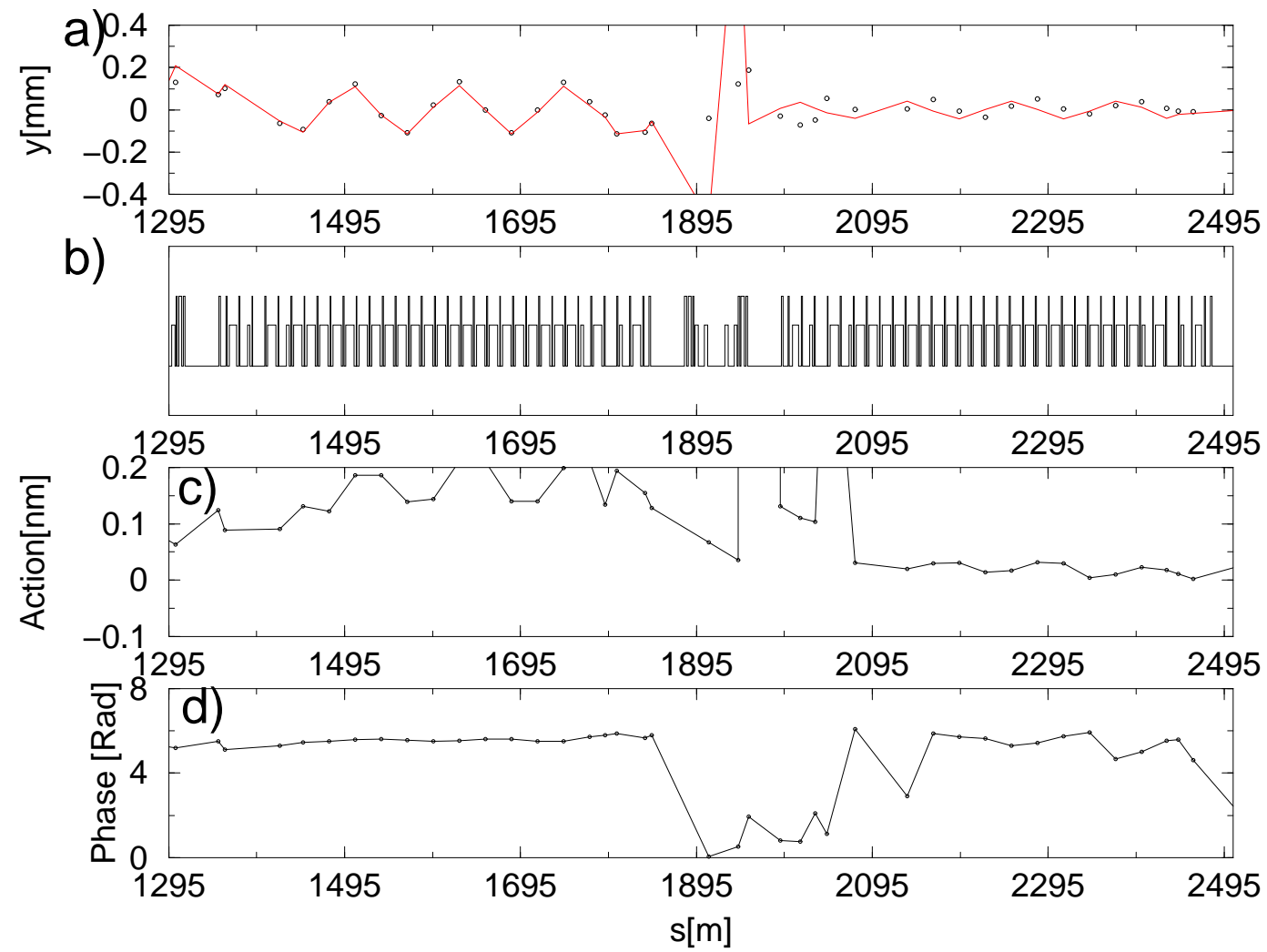

Figure E.1: a) Orbit measurements and corresponding modeling in two RHIC arcs. Each arc is fitted independently. b) Lattice representation of two RHIC arcs. The arcs correspond to the group of bars at each side of the smaller group which, in turns, represent the triplet magnets at the IR's. c) Action analysis of the orbit in a). d) Phase analysis of the orbit in a). 


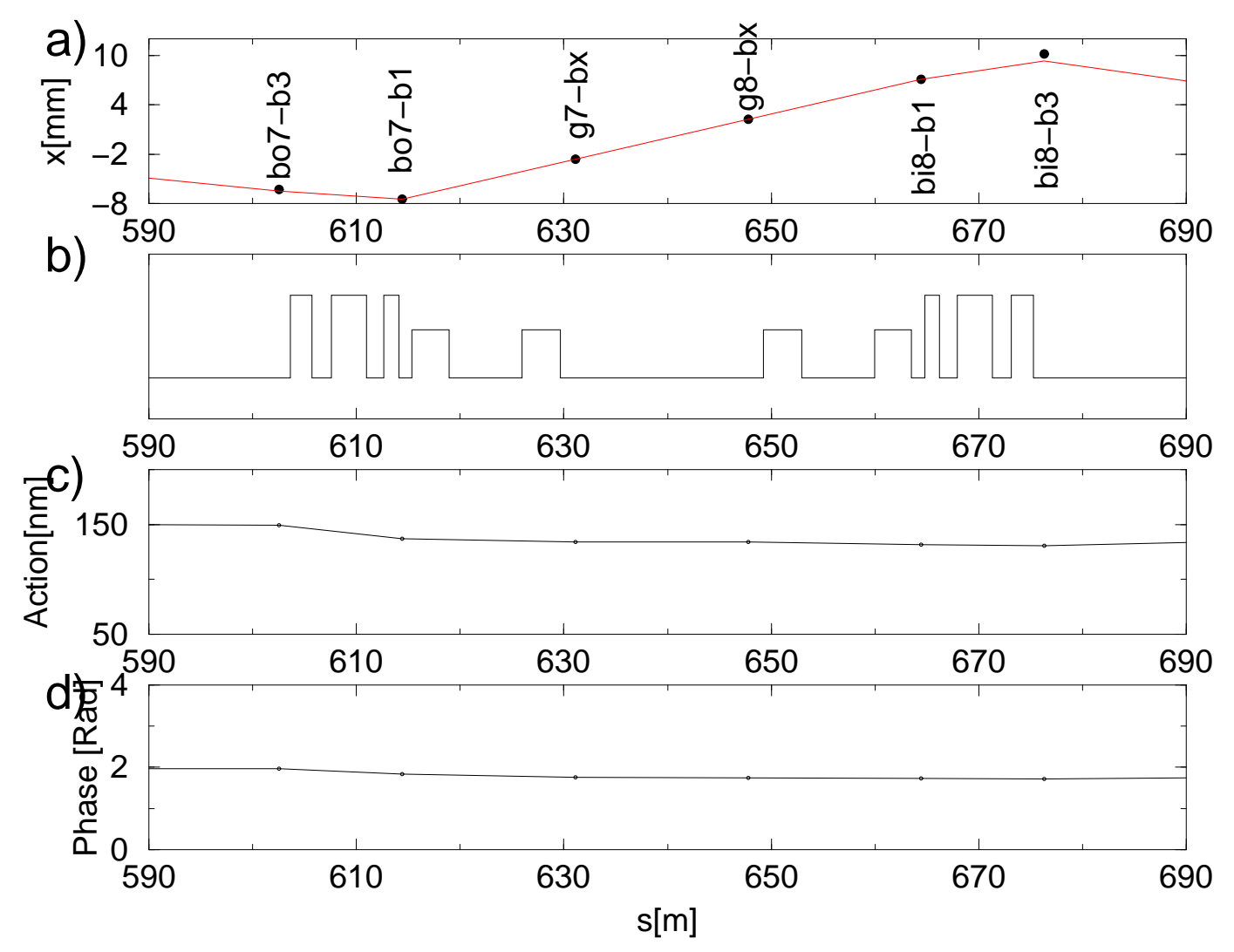

Figure E.2: a) Detail of a difference orbit showing all six BPMs used at the 8 o'clock RHIC IR. The central BPMs (bxs) are necessary to make individual estimates of the triplet errors. The b3s BPMs are used to estimate the beam positions at the correctors. If any of these BPMs fails, the closer b1 will be used instead. b) Left and right quadrupole triplets of the 8 o'clock RHIC IR (long bars). The short bars represent the Dx magnets used to control the collision of the beams. c) Action analysis of the orbit in a). b) Phase analysis of the orbit in a). 


\section{Appendix F}

\section{Skew Quadrupole Correctors}

The skew quadrupole correctors used at RHIC IRs are small quadrupoles that have been rotated 45 degrees counterclockwise as seen from the downstream face of the magnet (see Fig. F.1). The skew quadrupole can be turn on by a software program called the Ramp Editor Manager. The values use by the Ramp Editor Manager to set the skew quadrupole correctors, $s k_{r e}$, have the following relation with the physical quantities of the skew quadrupole corrector:

$$
s k_{r e}=\frac{B^{\prime}}{B \rho} \Delta s
$$

The sign convention here is important. A positive value of $s k_{r e}$ will produce the configuration of magnetic lines shown in Fig. F.1. Keeping the previous convention the magnetic kick, for example, in the $\mathrm{x}$ plane generated after setting a skew quadrupole corrector to some value $s k_{r e}$ is given by:

$$
\Delta x^{\prime}=-s k_{r e} y
$$




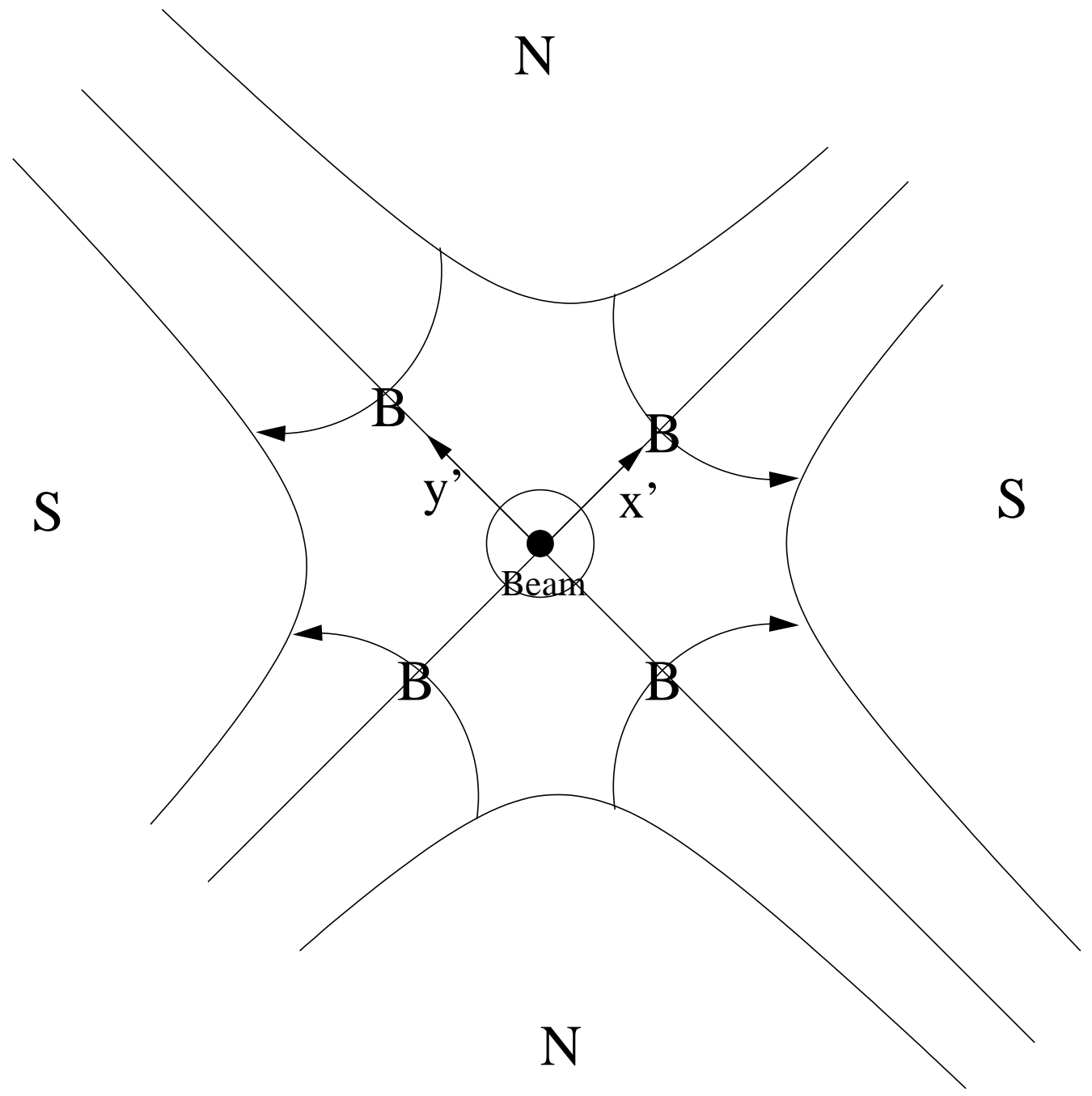

Figure F.1: View of the magnetic lines of a focusing quadrupole rotated 45 degrees in the counterclockwise direction from the downstream face of the magnet. 
since

$$
\Delta x^{\prime}=-\frac{B^{\prime}}{B \rho} \sin (2 \phi) \Delta s y
$$

where $y$ is the vertical position of the beam at place where the skew quadrupole corrector is located and $\sin (2 \phi)=1$ since the skew quadrupole is rotated $\pi / 4$ as shown in Fig. F.1.

It is neccesary to relate Eq. F.2 with the formalism develop in these book. Considering only the coupling term in Eq. 1.56 the magnetic kick, $\Delta x^{\prime}$, can also be written as:

$$
\Delta x^{\prime}=A_{1}^{c o r r} y
$$

where $A_{1}^{\text {corr }}$ is the skew quadrupole component due the corrector. Comparing Eq. F.2 and Eq. F.4 it is found that:

$$
A_{1}^{\text {corr }}=-s k_{r e}
$$

The calibration experiment in Chapter 8, and the Chapter 4 make use of Eq. F.5 to establish relations between measured values and the skew quadrupole corrector strengths. 


\section{Bibliography}

[1] E. D. Courant and H.S. Snyder Ann. of Phys. 3, 1-48 (1958).

[2] D. A. Edward and M. J. Syphers, in An Introduction to the Physics of High Energy Accelerators, ed. John Wiley \& sons, p. 67 and 69 (1993).

[3] M. Alonso and E. Finn, in Fundamental University Physics, Vol 1, 2nd Edition, ed. Adison Wesley (1967).

[4] J. Safranek, Experimental Determination of Storage ring Optics using Orbit Response Measurements, Nucl. Instrum. Methods Phys. Res., Sect A 388, 27 (1997).

[5] J. Irwin et al.,Model Independent Analysis,Phys. Rev. Lett. 82, 1684 (1999).

[6] Y. Cai et al.,Application of MODEL-INDEPENDENT ANALYSIS to PEP-II RINGS,Proceedings of the 2001 Particle Accelerator Conference, Chicago (2001).

[7] G. H. Golub and C. F. Van Loan, in Matrix Computations, The Johns Hopkins University Press,3rd ed. , Baltimore (1996). 
[8] P. Tenenbaum and T. O. Raubenheimer, Resolution and Systematic Limitations in Beam-based Alignment, Phys. Rev. ST Accel. Beams 3,052801 (2000).

[9] G. H. Hoffstaetter and F. Willeke, Beam Based Alignment of Interaction Region Magnets, http://www-library.desy.de/cgi-bin/showprep.pl?DESY02069,DESY, (2002).

[10] H. Grote, F. C. Iselin, The MAD Program, Ver. 8.19, Ed. European Organization for Nuclear Research (1996).

[11] S. Peggs and S. Tepikian, RHIC/AP/11, Brookhaven National Laboratory (1993).

[12] RHIC Design Manual, Brookhaven National Laboratory (2000).

[13] G. Guignard, Effets des champs magnetiques perturbateurs d'un synchrotron sur l'orbite fermee et les oscillations' betatroniques, ainsi que leur compensation, CERN 70-24 (1970).

[14] G. Guignard, The Closed Orbit Measurement as a Diagnostic Tool for Localisation and Correction of Misalignments in the ISR, CERN ISRBOM/80-21 (1980).

[15] M. Mynty and F. Zimmermann,in Beam Techniques - Beam Control and Manipulation USPAS Lectures at University of Chicago and Argonne National Laboratory,p. $23,(1999)$ 
[16] V. Ptitsyn, J. Cardona, F. Pilat and J. P. Koutchouk,Measurement and Correction of Linear Effects in the RHIC Interaction Regions, Proceedings of the 2001 Particle Accelerator Conference, Chicago (2001).

[17] F. Pilat, P. Cameron, V. Ptitsyn, J-P Koutchouck, Linear and Nonlinear Corrections in the RHIC Interaction Regions, Proceedings of EPAC 2002, Paris, France (2002).

[18] R. Tomás García, Direct Measurements of Resonance Driving Terms in the Super Proton Synchrotron (SPS) of CERN using Beam Position Monitors.,Doctoral Thesis, Universitat de Valencia, Spain, (2003).

[19] J. Cardona, V Ptitsyn, F. Pilat, Operational Experience on Linear Coupling Correction in the RHIC Interaction Regions, Snowmass Conference, Snowmass - Colorado (2001).

[20] P. J. Bryant and K Johnsen,in The Principles of Circular Accelerators and Storage Rings, Cambridge University Press, Cambridge,p. 100 (1993).

[21] D. A. Edward and M. J. Syphers, in An Introduction to the Physics of High Energy Accelerators, ed. John Wiley \& sons, p. 64 (1993).

[22] P. J. Bryant and K Johnsen,in The Principles of Circular Accelerators and Storage Rings, Cambridge University Press, Cambridge,p. 102 (1993).

[23] P. J. Bryant and K Johnsen,in The Principles of Circular Accelerators and Storage Rings, Cambridge University Press, Cambridge,p. 109 (1993). 
[24] F. Willeke and Ripken,in Methods of Beam Optics, Cornell Summer School, AIP Proceedings 184, and DESY 88-114 (1988).

[25] G. Ripken and F. Willeke, On the Impact of Linear Coupling on Nonlinear Dynamics, DESY 90-001 (1990).

[26] P. J. Bryant and K Johnsen,in The Principles of Circular Accelerators and Storage Rings, Cambridge University Press, Cambridge,p. 105 (1993).

[27] G. Guignard, The General Theory of All Sum and Difference Resonances in a Three-Dimensional Magnetic Field in a Synchrotron, CERN 76-06 (1976).

[28] J. Cardona, S. Peggs, T. Satogata, F. Pilat, V. Ptitsyn, Determination of Linear and Non Linear Components in RHIC Interaction Regions from Difference Orbit Measurements, Proceedings of EPAC 2002, Paris France(2002).

[29] P. Bryant, in A Simple Theory for Weak Betatron Coupling,CERN Accelerator School, CERN 89-05 (1989).

[30] D. A. Edward and M. J. Syphers, in An Introduction to the Physics of High Energy Accelerators, ed. John Wiley \& sons, p. 83 (1993).

[31] T. Satogata, in Coupling and Alignment of the PHENIX Experiment Solenoid in RHIC, RHIC/AP/19, BNL (1993). 
[32] P. R. Bevington and D. K. Robinson, in Data Reduction and Error Analysis for the Physical Sciences, ed. McGraw-Hill, p. 121 (1992).

[33] F. Pilat, P. Cameron, V. Ptitsyn, J-P. Koutchouk, in Linear and Nonlinear Corrections in the RHIC Interaction Regions, Proceedings of the EPAC 2002, Paris (2002).

[34] R. R. Wilson, in Radiological Use of Fast Protons, Radiology 47, 487 (1948).

[35] C. Ankenbrandt, T. Krock, L. Michelotti, S. Peggs and C. Schmidt, Pre-conceptual Design of a Proton Therapy Accelerator, FERMILAB-Pub92/136, (1992).

[36] F. T. Cole, in Accelerator Considerations in the Design of a proton therapy facility - a Reprise, Technical Report. Particle Acceleration Corporation. 4513 Cornell Avenue, Downers Grove, IL 60515. August 23, (1991).

[37] Eros Pedroni, Reinhard Bacher, Hans Blattmann, Terence Boehringer, Adolf Coray, Mark Phillips and Stefan Scheib, in Cancer Therapy with 200 MeV protons at PSI. Development of a Fast Beam Scanning Method and Future Plans for a Hospital based Facility, presented at the 1990 European Particle Accelerator Conference.

[38] S. Fukumoto et al. , in Present Status and Future of University of Tsukuba Proton Medical Research Center., Presented at the National Institute of 
Radiological Science International Workshop on Heavy Charged Particle Therapy and Related Subjects (NIRS: Chiba 260, Japan). July 4-5 1991. See also Sadayoshi Fukumoto, A dedicated accelerator for Proton Therapy at Tsukuba, Contributed to the 4th China-Japan Join Symposium on Accelerators for Nuclear Science and their Applications (Beijing, China). October 15-17 1990.

[39] S. Y. Lee, Accelerator Physics,World Scientific Publishing Co. ,(1999).

[40] S. Peggs (Editor), Pre-conceptual Design of a Rapid Cycling Medical Synchrotron, C-A/AP/6, BNL, (1999).

[41] S. Peggs, J. Cardona, M. Brennan, J. Kewisch, G. McIntyre, N. Tsoupas, M. Schillo, A. Todd, B. Ludewigt, N. Lockyer, RCMS - A Second Generation Medical Synchrotron, PAC, Chicago, 2001.

[42] S. Peggs, W. Meng, T. Roser, N. Tsoupas, J. Tuozzolo, in The RCMS dipole aperture and beam pipe, C-A/AP/9, Brookhaven National Laboratory, February 2000.

[43] J. Cardona, J. Kewisch, S. Peggs, Design of the RCMS Lattice Optics,EPAC,Paris - France, 2002.

[44] G. Coutrakon, J. Hubbard, J. Johanning, G Maudsley, T. Slaton and P. Morton, A performance study of the Loma Linda proton Medical Accelerator,Med. Phys., Vol 21, No 11,1994. 
[45] F. Cole, P. Livdahl, F. Mills and L. Teng, "Multi-station Proton Beam Therapy System" US Patent 4870 287, September, 1989.

[46] M. Umezawa et al., Beam Commisioning of the New Proton Therapy System for the University of Tsukuba, Proceedings of the 2001 Particle Accelerator Conference, Chicago, 2001.

[47] K. Hiramoto et al., a Compact Synchrotron for Cancer Treatment, Proceedings of 1997 Particle Accelerator Conference, Vancouver ,1997.

[48] M. Tadokoro, J.I. Hirota, K. Hiramoto, M. Umezawa, S. Kakiuchi, Y. Iwashita, A Noda, T. Shirai and M Inoue, $A$ combined function magnet for a compact synchrotron, particle accelerator conference, Vancouver, 1997.

[49] G.Coutrakon et al, Design Considerations for Medical Proton Accelerators, PAC'99, New York, 1999.

[50] S. Y. Lee, A multipole expansion for the field of vaccum chamber eddy currents, Nuclear Instruments and Methods in Physics Research A300 ,1991, pg 151-158.

[51] A. Dragt et al, Marylie 3.0 Uaer's Manual: A Program for Charged Particle Beam Transport Based on Lie Algebraic Methods, University of Maryland, College Park, Maryland, 1999. 
[52] A. Dragt and D. Abell, Symplectic Maps and Computation of Orbits in Particle Accelerators, Fields Institute Communications, Vol 10, 1996, pg $59-85$.

[53] A. Dragt, Lie Methods for Nonlinear Dynamics with Applications to Accelerator Physics, University of Maryland, College Park, Maryland, 1999.

[54] C. Gardner and S. Peggs (editors), Conceptual Design of the RCMS, To be published, Brookhaven National Laboratory, 2003, pg 30.

[55] C. Gardner and S. Peggs (editors), Conceptual Design of the RCMS, To be published, Brookhaven National Laboratory, 2003, pg 19.

[56] C. Gardner and S. Peggs (editors), Conceptual Design of the RCMS, To be published, Brookhaven National Laboratory, 2003, pg 46.

[57] S. Peggs, Some Aspects of Machine Physics in the Cornell Electron Storage Ring, Cornell University, 1981.

[58] J. Cardona, S. Peggs, T. Satogata, R. Tomas, Action and Phase Analysis to Determine Sextupole Errors in RHIC and the SPS., submitted to PAC'03, Portland, Oregon, 2003.

[59] J. Cardona, D. T. Abell,S. Peggs, Studies of Sextupole components generated by Eddy Currrents in the Rapid Cycling Medical Synchrotron,submitted to PAC'03, Portland, Oregon, 2003. 
[60] H. Wiedemann, Particle Accelerator Physics,Springer-Verlag, V 1-2, (1993). 